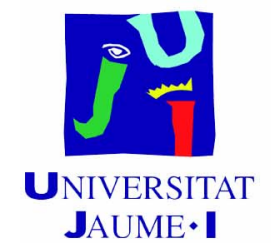

Instituto Interuniversitario de Desarrollo Social y Paz

DOCTORADO INTERNACIONAL EN ESTUDIOS

DE PAZ, CONFLICTOS Y DESARROLLO

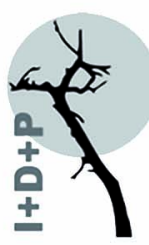

Tesis Doctoral

Open Source and Living Systems

Study and Design of an Open Source Hydro Turbine

for Remote Area Electrification

Autor:

Ino David Fleischmann

Supervisores:

Dr. Victoria Fontan

Dr. César Fernández Fernández

Selva Negra, Alemania - Invierno 2017 
There are no Passengers on Spaceship Earth. We are all Crew.

- Marshall McLuhan 


\section{Abstract}

\section{Open Source and Living Systems}

Study and Design of an Open source Hydro Turbine For Remote Area Electrification

Ino D. Fleischmann

This dissertation investigates the approach of open source hardware and its potential for a "post-growth" transformation of society. Beginning with the assessment of the global crisis it becomes clear that our energy demanding consumption- based lifestyle is clashing with limited resources in an limited system, called planet earth. It seems that we are living in a global system based on growth, where the economic system is forcing us to exploit the earth and its nature. While facing global environmental challenges like deforestation, pollution of air and water, dying species and melting glaciers we develop even more sophisticated technologies for exploitation, wage wars to conquer and protect resources and increase the production of waste without any ideas how to deal with it in the future.

A way for a post growth society can be found in the approach of open source. Open source in this context means sharing and collaboration instead of competition and growth. The dissertation investigates the paradigm shift attached to it and explores the ideas of open source, compares them with living systems in nature and contributes to the open source movement. This is done in the field of rural electrification. The thesis elaborates the potential and necessity of rural electrification with renewable energy. Therefore it compares renewable energy technologies from the perspective of open source applicability.

The practical output hereby is the design of an open source licensed hydro power turbine, called "Pico Cross Flow". Its simplified design is primarily meant for remote area 


\begin{abstract}
electrification and local manufacturing in the Global South with a power output of up to five kilowatts. This dissertation covers the hydraulic and mechanical design as well as the manufacturing of a prototype and the testing at the hydrodynamic laboratory of the University of Vienna. The Pico Cross Flow turbine is the practical outcome, based on the theoretical part of the dissertation.
\end{abstract}




\section{@creative}

Attribution-Noncommercial-Share Alike 4.0 International (CC BY-NC-SA 4.0)

This work is licensed under the Creative Commons Attribution-Noncommercial-Share Alike 4.0 International License. To view a copy of this license, visit https://creativecommons.org/licenses/by-nc-sa/4.0/ or send a letter to Creative Commons, 171 Second Street, Suite 300, San Francisco, California, 94105, USA.

You are free to:

Share - copy and redistribute the material in any medium or format

Adapt - remix, transform, and build upon the material

The licensor cannot revoke these freedoms as long as you follow the license terms.

Under the following terms:

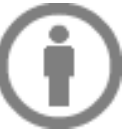

Attribution - You must give appropriate credit, provide a link to the license, and indicate if changes were made. You may do so in any reasonable manner, but not in any way that suggests the licensor endorses you or your use.

NonCommercial - You may not use the material for commercial purposes.

ShareAlike - If you remix, transform, or build upon the material, you must distribute your contributions under the same license as the original.

With the understanding that:

- Waiver - Any of the above conditions can be waived if you get permission from the copyright holder.

- Other Rights - In no way are any of the following rights affected by the license:

- Your fair dealing or fair use rights;

- The author's moral rights;

- Rights other persons may have either in the work itself or in how the work is used, such as publicity or privacy rights.

- Notice - For any reuse or distribution, you must make clear to others the license terms of this work. The best way to do this is with a link to

https://creativecommons.org/licenses/by-nc-sa/4.0/. 


\section{Table of Contents}

Abstract III

License $\quad$ V

Table of Contents $\quad$ VI

List of Abbreviations and Acronyms IX

Index of Charts, Figures, Photos and Tables I X

1 Introduction 1

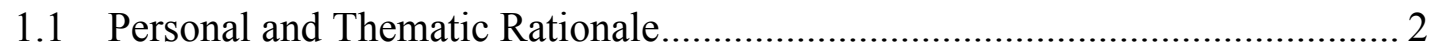

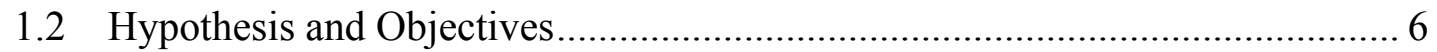

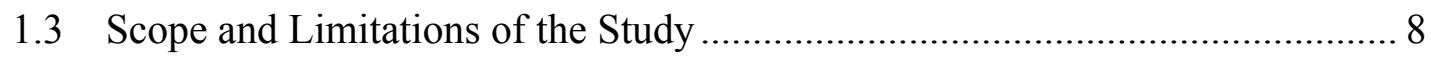

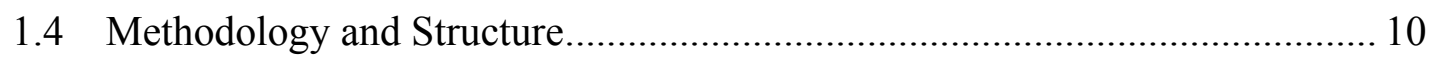

2 Facing the Fire - Assessment of the Global Crisis 16

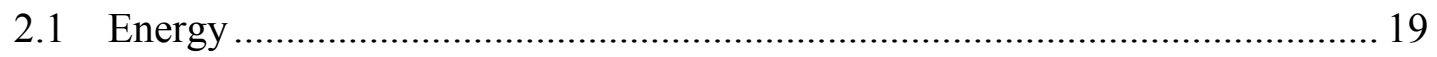

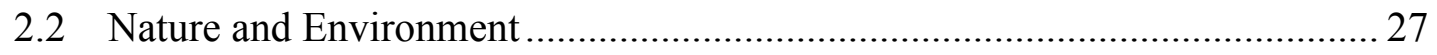

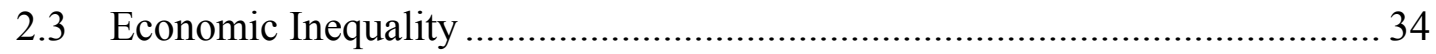

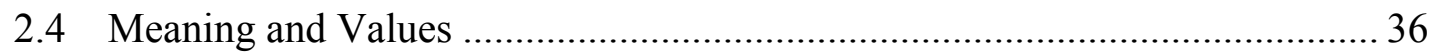

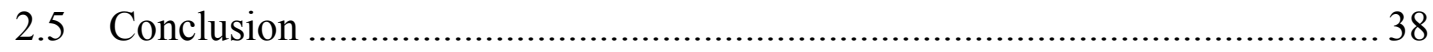

3 Paradigms $\quad \mathbf{4 0}$

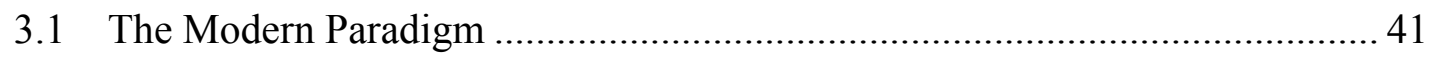

3.2 A systemic, organic Paradigm based on Life.............................................. 52

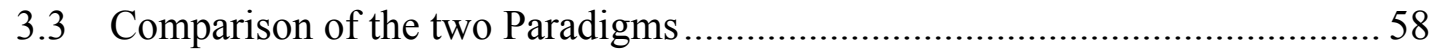

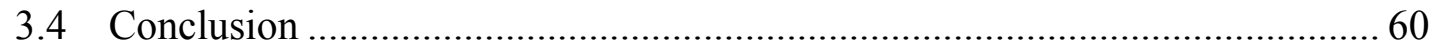

4 Open Source and Living Systems

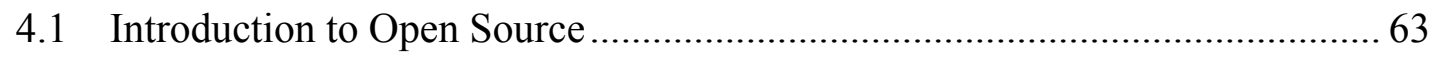

4.1.1 Open Source Software 66

4.1.2 Open Source Hardware $\quad 70$

4.1.3 Open Source Hardware Projects 75

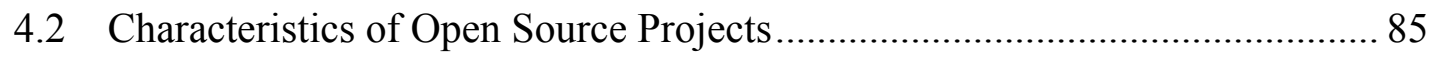

4.2.1 Dynamic Living Systems $\quad 85$

4.2.2 The Strange Attractor $\quad 89$

$\begin{array}{ll}\text { 4.2.3 New Communication Technology and Networks } & 90\end{array}$

4.2.4 Innovation, Diversity and Creativity 92

4.2.5 Self-Organization and Do-Ocracy 94

4.2.6 Personal Motivation and Meaning $\quad 97$

4.3 A Collaborative Society? - Some Observations ............................................ 99 
4.3.1 Third Industrial Revolution and Collaborative Economy 100

4.3.2 Peer Production and Distributed Enterprise 105

4.3.3 Appropriate Technology 2.0 109

4.4 Conclusion and Transition to Renewable Energy ........................................ 110

5 Renewable Energy and Rural Electrification 112

5.1 Population Growth, Energy Consumption and Electrical Distribution ......... 112

5.2 Rural Electrification - Outlook and Potential ............................................. 122

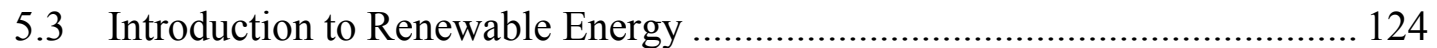

5.4 Technical Options for Rural Electrification................................................ 129

$\begin{array}{lll}5.4 .1 & \text { Wind Energy } & 129\end{array}$

$\begin{array}{ll}\text { 5.4.2 Biomass Energy } & 141\end{array}$

$\begin{array}{lll}5.4 .3 & \text { Solar Energy } & 145\end{array}$

$\begin{array}{ll}\text { 5.4.4 Hydropower } & 151\end{array}$

5.5 Technology Comparison and Feasibility Matrix ....................................... 159

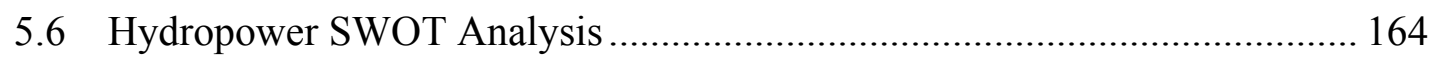

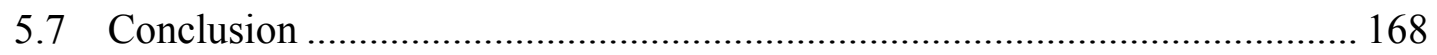

6 Feasibility of an Open Source Hydro Turbine 169

6.1 Turbine Types and Technical Evaluation ................................................... 169

$\begin{array}{lll}\text { 6.1.1 The Propeller and Kaplan Turbines } & 176\end{array}$

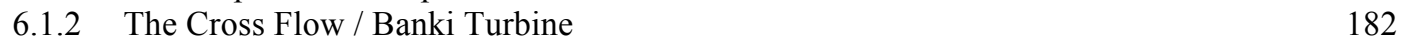

$\begin{array}{lll}\text { 6.1.3 Pumps as Turbines } & 187\end{array}$

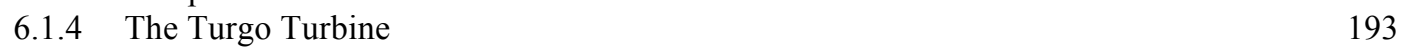

6.1.5 The Pelton Turbine 195

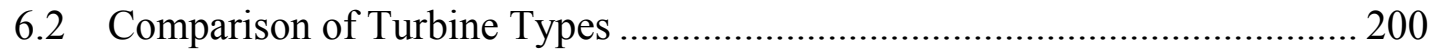

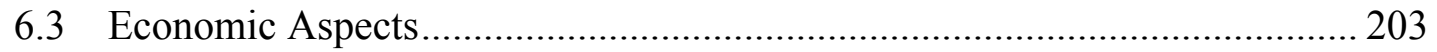

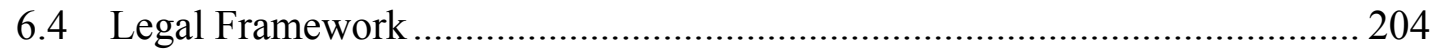

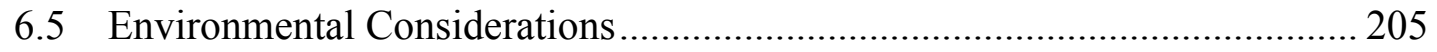

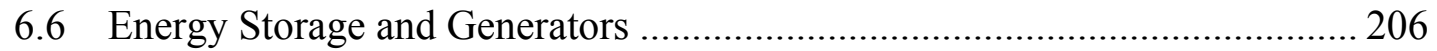

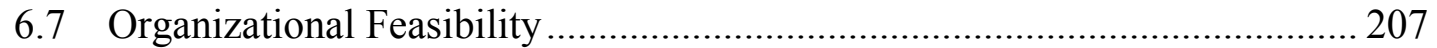

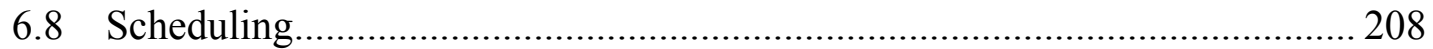

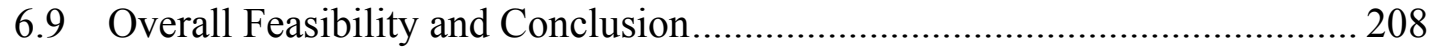

7 Practical Application: Pico Cross Flow 210

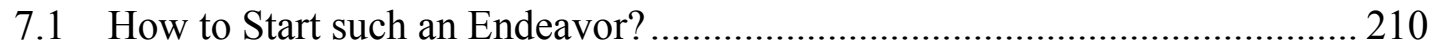

7.2 The Pico Cross Flow Turbine, an Open Source Hardware Design................ 213

$\begin{array}{lll}7.2 .1 & \text { Hydraulic Calculation and Design } & 214\end{array}$

$\begin{array}{ll}7.2 .2 & \text { Manufacturing a Prototyp } \\ 7.223\end{array}$

$\begin{array}{ll}\text { 7.2.3 Hydraulic Testing of the Prototype } & 226\end{array}$

7.2.4 The Pico Cross Flow Files - A Comprehensive Documentation 228

7.3 Challenges, Next Steps and Outlook ........................................................ 231 
9 List of References

10 List of Physical Units $\quad 250$

11 Resumen en Castellano $\quad 251$

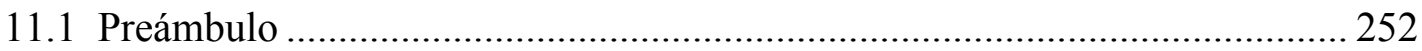

11.2 Objetivo de la Investigación y Pregunta Central ......................................... 257

11.3 Metodología y Estructura de la Disertación..................................................... 259

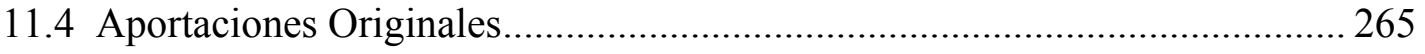

11.5 Desafios y Futuras Líneas de Investigación................................................. 267

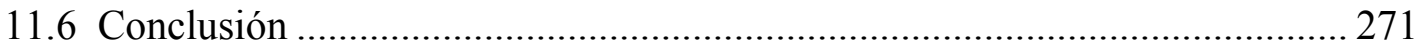

12 Appendix $\quad 274$

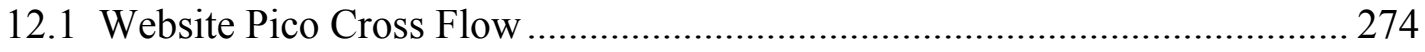

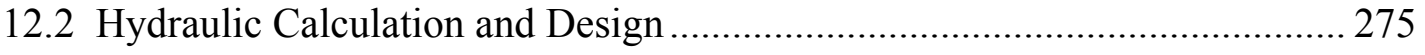

12.3 Pico Cross Flow Manufacturing Photos ....................................................... 280

12.4 Pico Cross Flow 3D Model and Drawings ................................................. 282

12.5 Pico Cross Flow Prototype Turbine Photos ................................................. 284

12.6 Hydraulic Testing at the Technical University Vienna Photos..................... 285

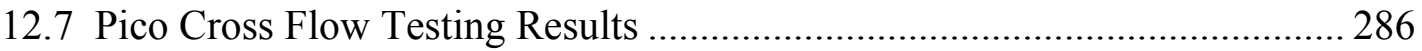




\section{List of Abbreviations and Acronyms}

\begin{tabular}{|c|c|}
\hline Acronym & Description \\
\hline ARE & Alliance for Rural Electrification \\
\hline AT & Appropriate Technology \\
\hline BHA & British Hydropower Association \\
\hline BOM & Bill of Materials \\
\hline CAS & Complex Adaptive Systems \\
\hline $\mathrm{CE}$ & Conformité Européenne \\
\hline $\mathrm{CI}$ & Corporate Identity \\
\hline CIP & Continuous Improvement Process \\
\hline $\mathrm{CNC}$ & Computer Numeric Control \\
\hline CSP & Concentrated Solar Power \\
\hline CMS & Content Management System \\
\hline $\mathrm{CO}_{2}$ & Carbon Dioxide \\
\hline DIY & Do It Yourself \\
\hline EnDev & Energising Development \\
\hline ESHA & European Small Hydropower Association \\
\hline EWB & Engineers without Borders \\
\hline GDP & Gross Domestic Product \\
\hline GVCS & Global Village Construction Set \\
\hline IEA & International Energy Agency \\
\hline HAWT & Horizontal Axis Wind Turbine \\
\hline ITDG & Intermediate Technology Development Group \\
\hline LDC & Least developed countries \\
\hline LPI & Living Planet Index \\
\hline OS & Open Source \\
\hline OSE & Open Source Ecology \\
\hline $\mathrm{OSH}$ & Open Source Hardware \\
\hline OSI & Open Source Initiative \\
\hline OSS & Open Source Software \\
\hline
\end{tabular}


List of Abbreviations and Acronyms

\begin{tabular}{|l||l|}
\hline OSHWA & Open Source Hardware Association \\
\hline PAT & Pump as Turbine \\
\hline PPS & PicoPV Systems (photovoltaics) \\
\hline PV & Photovoltaic \\
\hline RS & Rating System \\
\hline SHS & Classical Solar Home System \\
\hline SRS & Solar Residential System \\
\hline SWOT Analysis & Strengths Weaknesses Opportunities Threats Analysis \\
\hline TIR & Third Industrial Revolution \\
\hline UN & United Nations \\
\hline UNDP & United Nations Development Program \\
\hline UNPD & United Nations Populations Divison \\
\hline VAWT & Vertical Axis Wind Turbine \\
\hline WF & Weighting Factor \\
\hline WWF & World Wide Fund for Nature / World Wildlife Fund \\
\hline
\end{tabular}




\section{Index of Charts, Figures, Photos and Tables}

\begin{tabular}{|c|c|c|}
\hline Identification & Description & Page \\
\hline [2.1] & $\begin{array}{l}\text { Length of Time Plutonium must be isolated from the } \\
\text { Environment }\end{array}$ & 26 \\
\hline [2.2] & Humanity's Ecological Footprint & 28 \\
\hline$[2.3]$ & Global Living Planet Index & 32 \\
\hline [2.4] & Global Trends in the State of Marine Fish Stock $1974-2011$ & 33 \\
\hline [2.5] & Global Adult Population and Share of Wealth 2015 & 35 \\
\hline [3.1] & Comparison Table of the two Paradigms* & 59 \\
\hline [4.1] & Web Server Developers: Market Share of active Sites & 69 \\
\hline [4.2] & OHANDA Label & 74 \\
\hline [4.3] & miniCNC from Timelab & 79 \\
\hline [4.4] & Thing-O-Matic from MakerBot Industries & 80 \\
\hline$[4.5]$ & Tractor and Global Village Construction Set from OSE & $83 / 84$ \\
\hline [4.6] & Windows Family Tree & 92 \\
\hline [4.7] & Linux Distribution Timeline & 93 \\
\hline [5.1] & Energy total final Consumption by Area & 113 \\
\hline$[5.2]$ & Energy Consumption in New York and Sub-Saharan Africa & 115 \\
\hline$[5.3]$ & World Population 2012 & 116 \\
\hline [5.4] & Electrification Rate by economic Power & 117 \\
\hline$[5.5]$ & $\begin{array}{l}\text { Share of People without Access to Electricity in Developing } \\
\text { Countries }\end{array}$ & 118 \\
\hline$[5.6]$ & Electrification Rate by Region & 119 \\
\hline$[5.7]$ & Urban und rural electrification Rates & 119 \\
\hline [5.8] & National, urban and rural Electrification Rates & 120 \\
\hline [5.9] & $\begin{array}{l}\text { Overview of renewable Energy Sources and Transformation } \\
\text { Processes* }\end{array}$ & 125 \\
\hline$[5.10]$ & Configuration of Wind Turbines* & 132 \\
\hline$[5.11]$ & Electrical schematic Drawing of a small Wind Turbine* & 132 \\
\hline$[5.12]$ & Bernoulli Effect* & 134 \\
\hline [5.13] & Accelerated Airflow lowers Pressure* & 134 \\
\hline
\end{tabular}




\begin{tabular}{|c|c|c|}
\hline$[5.14]$ & Comparison of Rotor Types & 137 \\
\hline$[5.15]$ & Wind Power Design Examples & 139 \\
\hline$[5.16]$ & Transformation Paths to generate electric Power from Biomass & 142 \\
\hline$[5.17]$ & Transformation Paths to generate electric Power from Biogas & 143 \\
\hline [5.18] & Schematic view of an off-grid PV-System* & 147 \\
\hline$[5.19]$ & $\begin{array}{l}\text { Concentrator / Receiver Systems for Solar Thermal } \\
\text { Technologies }\end{array}$ & 149 \\
\hline$[5.20]$ & GoSol solar Concentrator and its Replication in Pakistan & 150 \\
\hline$[5.21]$ & The Hydrologic Cycle* & 152 \\
\hline$[5.22]$ & Hydro Electric Power Generation & 153 \\
\hline [5.23] & Euler's Turbine Equation & 155 \\
\hline$[5.24]$ & System Efficiency for a Scheme running at full Design Flow & 156 \\
\hline$[5.25]$ & Efficiency in Energy Generation & 157 \\
\hline$[5.26]$ & $\begin{array}{l}\text { Advantages / Disadvantages of rural Electrification } \\
\text { Technologies* }\end{array}$ & 159 \\
\hline$[5.27]$ & Technology Selection* & 162 \\
\hline$[5.28]$ & SWOT Analysis Matrix for open source Hydropower* & 166 \\
\hline$[6.1]$ & Schematic View of a low and high head Power House & 170 \\
\hline$[6.2]$ & Classification of Turbines* & 171 \\
\hline$[6.3]$ & Specific Speed for various Turbine Types & 172 \\
\hline$[6.4]$ & Functional Range of different Water Turbines by Escher-Wyss & 173 \\
\hline$[6.5]$ & Typical Turbine Efficiency by Type & 175 \\
\hline$[6.6]$ & Kaplan Turbine with different Blade Angles & 177 \\
\hline$[6.7]$ & Axial Turbine fabricated in León & 179 \\
\hline$[6.8]$ & Installation of axial "Simplex" Turbine in León Nicaragua* & 180 \\
\hline$[6.9]$ & Installation of axial "Simplex" Turbine in León Nicaragua* & 181 \\
\hline$[6.10]$ & Cross Flow working Principle & 183 \\
\hline$[6.11]$ & Michell Banki Turbine fabricated in Mozambique & 183 \\
\hline$[6.12]$ & Michell Banki Turbine fabricated in Peru, installed in Nicaragua & 183 \\
\hline$[6.12]$ & Cross Flow Design Firefly & 184 \\
\hline$[6.14]$ & A Cross Flow installation from Peru & 184 \\
\hline$[6.15]$ & Turbine and Generator connected to Penstock & 186 \\
\hline
\end{tabular}




\begin{tabular}{|c|c|c|}
\hline$[6.16]$ & Dam and Intake of a Cross Flow Installation* & 186 \\
\hline$[6.17]$ & $\begin{array}{l}\text { Working Principle Sketch and Comparison of Pumps and } \\
\text { Turbines }\end{array}$ & 188 \\
\hline$[6.18]$ & Francis Turbine and PAT directly connected to Generator & 189 \\
\hline [6.19] & PAT Principle Sketch & 190 \\
\hline$[6.20]$ & PAT Efficiency Curve & 190 \\
\hline$[6.21]$ & PAT Installation in Honduras* & 192 \\
\hline$[6.22]$ & PAT with separate Generator* & 192 \\
\hline$[6.23]$ & Turgo Runner & 193 \\
\hline$[6.24]$ & Water Flow through a Turgo Turbine & 194 \\
\hline$[6.25]$ & Water Jet impinging on the Rotor of a Turgo Runner & 194 \\
\hline$[6.26]$ & A Chinese Design of a 300W Pico Turgo Turbine & 194 \\
\hline$[6.27]$ & Pelton Turbine Working Principle & 196 \\
\hline$[6.28]$ & Pelton Turbine fabricated in Nicaragua* & 196 \\
\hline$[6.29]$ & Flow of Water in a Pelton Runner & 196 \\
\hline$[6.30]$ & Pelton Water Flow Theory & 196 \\
\hline$[6.31]$ & Three Jet Pelton Turbine with Flywheel, fabricated in Honduras* & 198 \\
\hline$[6.32]$ & Comparison of different hydro Turbines* & 201 \\
\hline$[6.33]$ & Comparison of HydroPower Costs & 203 \\
\hline$[7.1]$ & Application Range for Pico Cross Flow* & 213 \\
\hline$[7.2]$ & Gross Head of Hydro Power Scheme & 216 \\
\hline$[7.3]$ & Flow Admission Area of a Cross Flow Turbine & 218 \\
\hline$[7.4]$ & Equations of Geometrical Relationships between Parameters & 219 \\
\hline$[7.5]$ & Construction of Blade Geometry & 219 \\
\hline$[7.6]$ & Velocity Triangles in a Runner with peripheral Blades & 221 \\
\hline$[7.7]$ & Cross Section of Flow at the Inlet & 221 \\
\hline$[7.8]$ & Design of the Logarithmical inlet Spiral & 221 \\
\hline [7.9] & Assumptions of the Turbine Layout & 222 \\
\hline$[7.10]$ & Results of the Turbine Layout & 222 \\
\hline$[7.11]$ & Manufacturing of the Prototype in Managua & 224 \\
\hline$[7.12]$ & Turbine test rig at the University of Vienna & 227 \\
\hline
\end{tabular}


List of Charts, Figures, Photos and Tables

\begin{tabular}{|l|l} 
[7.13] & Different Revolutions related to Water Head
\end{tabular}

* author's illustration or photo 


\section{Introduction}

We find ourselves living in a global system based on growth, where the economic system is forcing us to exploit Earth and its nature. While facing global environmental challenges like deforestation, pollution of air and water, dying species and melting glaciers, we develop even more sophisticated technologies for exploitation, wage wars to conquer and protect resources, and increase the production of waste without any ideas on how to deal with it in the future.

Our current way of life and economic system are demanding more and more energy. Fossil fuels and nuclear energy seem to support our modern, energy-demanding consumer lifestyle. These energy resources are made available through a linear and highly centralized, top-down hierarchic structure.

The complex industrial systems, both organizational and technological, are the main driving force of global environmental destruction, socioeconomic inequality and social dissatisfaction. We are selling Mother Earth's resources with no conscience toward its effects on the environment. However, growth in a finite system necessarily has to reach an end. Even more alarming, the United Nations projects a world population growth "from 6.1 billion in 2000 to 8.9 billion in 2050, increasing by 47 per cent” (United Nations, 2004).

All of this has to be seen in the frame of a shifting paradigm, especially in an information and energy shift. We are living in an energy transition from a fossil-fuel age (coal, oil and natural gas) to a solar age, powered by renewable energy from the sun. At the same time, we are witnessing a revolution in communication technology, which connects human beings on this planet as never before. These transitions will and already have a profound impact on our lives, societies and social structures. 
How does this interdisciplinary shift, perhaps a shift of awareness, affect our economic and social systems? How do we organize and motivate ourselves? If personal material growth is not our driving force, what is it then?

This research project explores the society-transforming potential of the ideas of open source (OS), especially as related to open source hardware (OSH). The open source approach is investigated from different angles and then practically applied in the field of remote area electrification through an open source hydropower turbine.

\subsection{Personal and Thematic Rationale}

When I decided to pursue an academic path in the field of Peace Studies, I struggled deeply with what I perceived as an inherent contradiction within myself: being a professional engineer, believing in the beauty and logic of complex structures and mechanisms, while feeling deeply connected to the idea of peace and its various manifestations. When I started the Masters program in Peace, Development, Security and International Conflict Transformation at the University of Innsbruck, as part of our introduction phase I presented myself to my new colleagues with a short bio of who I am, what I have done in the past and what I would like to do in the future. One of my fellow students replied to me: 'You don't have to worry about your unusual engineering background and your work in renewable energy. In the end, Peace Studies is all about transforming energy. You might like it." It took me some time, but I eventually realized the essential truth that lies in that statement; and it was this very essence that led me to the $\mathrm{PhD}$ program at Castellón, committed to this research. 
I assume that everything boils down to your intention. I grew up in a rather rural area, and from the time I was a child I have always had a strong connection to nature. When we were playing in the forest, carving nice wooden sticks, it was always the other children who had the biggest. I remember asking myself: Is it worth it to kill this plant just because I want to play with my knife? Mostly, I preferred to cut off a branch rather than decapitate the whole trunk of a little tree. I guess my awareness for the environment comes from that time. And I was always interested in technology and wanted to know how things work. As a child I played with all kinds of technical toys. It was great; there was so much to discover. I remember cutting off the cable of a cable-controlled car. My intention was to make it wireless remote-controlled, and in the process I realized that it does not work that easily. Cutting off the cable does not accomplish that objective, and even worse, it makes it not work at all.

After school, I studied mechanical engineering and entered into a physics-mathematics, scientific-linear path of education and thinking. I learned how technical things work, but the question of intention remained, especially after my studies, when I began working as an engineer in renewable energy (large hydropower). From a technical perspective, it was exciting, interesting, and challenging to design and physically bring a turbine runner of 150 tons to a site in the middle of nowhere, on a pure, natural section of a river where there are no roads. But I was also aware that the running machine, as well as the building and implementation process, had serious impacts on the environment. And I became aware that the main idea often was to make profit and satisfy investors, and not to provide electricity to people in need. I guess that the intention of an industrial employer is not necessarily the "best" technical or "best" sustainable solution, but a technical solution for "maximum profit" for the company. Furthermore, the competition among companies who actually pursue the same goal is strikingly problematic. In those huge projects, managing millions of Euros, 
many companies are involved. All of them work together, share responsibilities and want to build the same plant. Nevertheless, each party involved in a project also looks toward its own advantage. The goal to strive for is to make profit by adhering to the specific task the company is assigned and to be legally safe after the job is finished. The intention of cocreation and working together often becomes almost secondary. The lack of motivation to cocreate ideas was frustrating to me as a mindful engineer.

My experience with open source software development helped me to realize that the approach outlined above is pointing in a problematic and unsatisfying direction. I am convinced that the development of technology in an economic environment is driven in large part by a "wrong intention". To some extent, it denies the core idea and the benefits of humans working together, and secondly, impacts the environment in an irresponsible way. I started open source software development as a hobby. In this environment I was surrounded by likeminded people. People interested in improving a product because of their own experience with it. I felt that the driving force for development and improvement of the product was completely different, aligned with one's intention.

As my research topic to complete the program in peace studies, I chose rural electrification with renewable energy and started working in the field of rural electrification through small hydropower in Nicaragua and Honduras. I facilitated and technically supported the use of small-scale hydropower, which in this case had very little impact on the environment and primarily benefitted the people, who lacked access to energy. As part of my job, I stayed with communities, literally in the middle of nowhere, to support installation and accompany the electrification process. Having worked with people who are not connected to electricity "electrified" as I like to say - and instead live in profound connection with nature, offered me 
the unique possibility to research the energy effects on humans and social structures, and also investigate the relationships between people and nature. In these rural areas and communities, specific technical needs are visible. And it became obvious that very little "technology" is necessary to satisfy energy needs in rural areas.

I developed a technical approach to the topic, but I was never fully confident with it. In the process of this research, I went to two conferences that changed my perspective. One conference focused on decentralized energy supply, which enabled me to develop new perspectives on the field of development work. I had the impression that many well-dressed people tried to sell their solutions and products to other well-dressed people who offered them some money. It seemed that this process had not so much to do with the people in need, although they were shown on pictures in many PowerPoint slides. The other conference on open knowledge almost overwhelmed me, given the inspiring people, ideas and approaches I encountered. The energy and atmosphere at this conference were impressive and mirrored quite well the state of development in this field, where many things happen very fast these days. This context added the missing element and completed my topic: combining technical solutions with the approach of open source. This also offered me a unique avenue to blend the two fields I was working on over the years, and it touches strongly on the intention of creating a technical solution or product for rural electrification.

Taking this diverse background into consideration, my $\mathrm{PhD}$ research elaborates on the feasibility of small-scale Open Source Hydro Power solutions. It should be seen as a technical and social feasibility study, consisting of theoretical and practical parts. My motivation for choosing this topic is multi-dimensional. On one hand, I am interested in energy and rural electrification. Moving out of energy poverty and having access to (renewable) energy represent a basic need. It is technically fascinating to reduce physical 
working principles to their minimum, which is often more than enough to transform small amounts of energy. Rural electrification in less developed countries is a particularly intriguing and practical topic, affecting quality of life for many people in a very profound way. On the other hand, it is fascinating to see how the open source approach is offering a new mode of how people interact and create common goods at a global scale. This approach has been seen in software development and with Wikipedia ${ }^{1}$, for example. Many people virtually gather, work on a common problem and develop a common solution, which can be used by everyone. And this also starts to happen with hardware, where real products are developed on a global scale. Open Source Hardware for Rural Electrification is the combination of these two topics, creating a challenging and multidisciplinary research field. I am very happy to work in this field, combining an engineering perspective with a social, peace studies perspective.

\subsection{Hypothesis and Objectives}

The topic of this study is related to electrification, and especially, to rural electrification in remote areas in what are referred to as low-income economy countries. On the one hand, it compares the applicability of different technical renewable energy solutions, like bioenergy, wind power, solar concentrated power, photovoltaic and hydropower for remote area electrification. On the other hand, it offers a feasibility study analyzing the development and implementation of such technology. Therefore, a large part of this research focuses on the concept of open source hardware and decentralized manufacturing possibilities, which adopts the approach of open source software development to that of hardware. This approach can be

\footnotetext{
${ }^{1}$ Wikipedia is a free-access, free-content Internet encyclopedia, supported and hosted by the non-profit Wikimedia Foundation (Wikipedia, Retrieved April 05, 2016 from https://en.wikipedia.org/wiki/Wikipedia). 
seen as a postindustrial form of economy with a non-capitalist intention. It refers to a more organic, self-organized, collaborative and perhaps a more natural method of development. Furthermore, the approach is connected with the issue of communication using new media and networks, which is especially visible in the product development process.

The concrete research question here asks if small-scale renewable energy solutions can be developed and implemented with an open source approach, and which of the different technologies contains the most promising potential? Similarly, this study seeks to answer the questions of how and where to start such an endeavor and also investigates and accompanies its practical realization.

By answering the questions above, I want to elaborate the possibility of developing renewable energy solutions that draw on methods of collaboration and sharing technology without giving in to financial motivation, and without taking the risk of imposing a certain technology on others. The motivation comes from the inside, as intrinsic motivation, which means that the inducement to change or act is based on an inherent desire. This boils down to one's intention. Open source seems to be the right tool to carry out this approach, as one of its essential ideas is that of self-organization and creativity.

\section{Hypothesis:}

The hypothesis, which is the foundation of this study, can be framed as follows: How is open source hardware feasible and how might it be a social model, beyond capitalist, growth and competition oriented societies, that fosters collaboration, openness and sharing? How does a pico hydropower turbine contribute to this approach? The hypothesis is practically applied and researched in the field of rural electrification. 


\section{Objective of the Research:}

The objective of this doctoral project is to research how the open source approach might be an alternative to our "consumer lifestyle" and how it impacts social structures and our (re)connection to nature. Different values like awareness, intention and motivation would be at the core of this approach. Global cooperation, mutual benefit and compassion instead of competition, greed and growth will be central to a post-materialistic form of society.

However, as an engineer working in remote area electrification with renewable energy, a large part of the research will take place in the context of physical science.

This component of the project, a feasibility study for open source hardware and rural electrification, elaborates on physical principles and technical solutions for renewable energy transformation. The final objective of this component is to build the scientific groundwork for the development of a product for rural electrification, which is then to be orchestrated in the last part of the study - the practical application and design of a hydropower turbine. This final part focuses on the design and manufacturing of a pico hydropower turbine from the perspective of OSH and can be seen as the "practical objective"; a contribution to the open source movement in renewable energy.

\subsection{Scope and Limitations of the Study}

The scope of this study focuses on two main areas: technical and social. The technical part concentrates on remote area electrification, mainly applied in so-called low-income economy countries, where national electricity grids are not available within the whole country. Remote area electrification, for our purposes, means "off-grid", decentralized energy supply, and/or so-called island solutions. Locally transformed energy is saved in battery banks and not connected to a bigger network. The production of energy and its consumption are basically 
located in the same place. Consequently, no transport or transmission of electricity is involved. Another limitation of the technical focus is that this study will only cover renewable energy sources provided by the sun's energy. This technical component will be conducted as a purely technical, straight-forward analysis of what is possible and how it is done; an illustration of the "state-of-the-art" technology for rural electrification, which also includes aspects of designing an open source "do-it-yourself construction kit". More precisely, the technical chapters do not discuss technical details or elaborate technical solutions in depth. However, for understanding the concepts behind renewable energy, these chapters give an overview of the physical background of each technology, an important basis for understanding the actual feasibility study.

As a practical application, the final chapter offers a technical design of a hydro turbine, which can be replicated with very little effort. The technical scope of the turbine design is limited to the mechanical side. The electrical side, along with the governing and controlling system, are not covered in this research. These components may be commercially bought or contributed as part of another open source hardware project.

At the social level, this study will focus on the approach of open source, which is based on the concepts of collaboration and trust. Open source projects are based on a common sense of co-creation and intrinsic motivation. In this sense, it is a self-sustainable renewable energy solution for rural electrification. This part touches upon the influential ideas of dynamic networks and living systems, as well as self-organization and personal empowerment. Even if in some parts of the study the open source approach is named "implementation model", the focus of analysis will not rest on the implementation process itself. Since there is no data available thus far regarding this process, and given the fact that there are only a few known open source hardware projects targeting energy, this aspect would be rather difficult to 
research at this point. The implementation on location could only be researched as a second step, after machines are installed and data and experience are available on how it actually affects off-grid rural communities. Therefore, this study will primarily focus on the social aspects of co-creating and collaboratively developing technical solutions. In this study, the term "implementation model" refers more to the self-organized, bottom-up approach, which relates strongly to the intention of technical development and not to the linear process of technical implementation.

\subsection{Methodology and Structure}

This doctoral thesis consists of several parts, starting with the historical context, global challenges and transition period in which we live. It describes a possible paradigm shift, a social change in motivation and intention. There is a theoretical research section on the topic of consumption, social structures and our connection to nature, based primarily on classical literature research.

The technical part of the research is based on the method of a classical feasibility study. A feasibility study is a formalized written approach that evaluates the potential and practicability of a product, project or idea. It is a management tool, used as a basis for decision-making, mainly used for business ideas and technical questions. It gathers and compares useful facts and figures, which help to cover a question from different perspectives and angles. This process should consider all areas of an idea and ensure that all possible angles and facets of potential influence are covered. It aims at helping to identify objectively and rationally ascertainable strengths and weaknesses, as well as opportunities and threats. 
Both obstacles and opportunities become visible and should also be documented. The feasibility study carried out in this research will assess and analyze the following questions:

- What are the needs and potential of rural electrification?

- Which of the proposed technologies are practical?

- What is the legal framework?

- Has it already been done? Do patents affect the idea?

- Are there other people potentially interested and what are their ideas?

- Are there other people who could be potential co-operators?

- Is it technically feasible?

These questions correlate with the main categories for a classical feasibility study - the socalled TELOS categories (Overton, 2007: 6). As such, the questions are elaborated within these five categories in their respective subchapters:

○ Technological feasibility

- Economic feasibility

- Legal feasibility

- Organizational feasibility

○ Scheduling feasibility

Technological feasibility covers all aspects of the technical practicability of small-scale renewable energy solutions. An economic feasibility study, also called cost/benefit analysis, is carried out by businesses to find out if a product is able to generate an expected and measurable profit. A company has to weigh costs versus benefits before proceeding in an accurate and precise way. In the frame of this study, the economic feasibility will not be covered in depth since profit-making is not the purpose or goal of open source projects. Nevertheless, the technical solution will be analyzed in regards to the costs of the transferred energy. The legal feasibility covers the question of whether a technical solution is conflicting with already existing technical solutions and patents of established companies. Operational feasibility generally covers if and how an organization is able to realize an idea, including manpower, equipment and tools. Also, this aspect will only be touched on slightly in this 
study, since this is not the organizational form of open source projects. The schedule feasibility covers the timeline or roadmap of such a project and its technical development time. This is a crucial factor in businesses that seek to place their products on the market faster than their competitors. There are additional aspects of schedule feasibility, which touch on the usefulness of a project. For example, if the process of developing and realizing a project evidently takes too long, it might lose its usefulness and fail. Schedule feasibility allows for estimating and measuring the reasonability of a project timeline. This will also be elaborated further in strategies discussed.

The evaluation of the required facts and figures to answer the questions raised above will be made through a Strengths - Weaknesses - Opportunities - Threats (SWOT) analysis based on a feasibility analysis matrix: "The SWOT analysis is the foundation for developing your strategy and tactics that then become the road map for writing your business operating plan" (Business Strategies, 2012). It will depict a previously selected technology, and by indicating its advantages and disadvantages, it helps the decision-making process. It is also the base for articulating a strategy and successively creating an action plan.

The technology considered in the SWOT analysis are chosen based upon the results of the feasibility matrix analysis. The matrix defines the key factors used in the feasibility evaluation. Each factor has its particular weight and is evaluated according to each technology. In the end, the total of the calculated weight will define the order of the evaluated system and the evaluated technologies.

This research is followed by a comparison of different hydro-turbine types. All of the physical and technical principles are presented. These technical options are then elaborated and compared through different categories and benchmarked through the perspective of open source hardware and DIY (do it yourself) feasibility. 
Stemming from the theoretical result of this research, the practical application component comprises the second part of the thesis. This covers the experiences of the open source community itself, formed by the nonprofit NGO Global Anchor. I formed Global Anchor as a legal entity in the very beginning of the technical research. The following lines are from the Global Anchor website and state the vision and mission of the NGO:

Global Anchor is a non-profit organization, supporting the development of Open Source Hydropower solutions for Rural Electrification. We want to provide robust, low-maintenance, low-cost, highly-capable and safe machines that will increase the proliferation of hydroelectricity and make a significant contribution to remote area electrification in a sustainable way. As part of the Open Source movement we hope to simplify the creation and sharing of micro hydro power machines to satisfy energy needs and bring light to remote areas.

Our Vision is to empower people in rural / remote / off-grid areas to satisfy their energy needs in an environmental friendly, renewable and self-sustainable way. Our Mission is to develop pico hydropower solutions, make our knowledge accessible, encourage people to use this knowledge to build their own turbines (Do It Yourself Construction Kits) and thereby support rural electrification (Global Anchor, 2013).

With Global Anchor, this research is framing a space for the development of a pico hydropower turbine and its necessary components. If you want to design and build your own hydroelectric site, understand it from ground up, and want to operate such a system yourself, you will find the information open and freely accessible on globalanchor.org. The Global Anchor Manuals will include the whole process of project implementation, from the initial steps (field measurements and site evaluation) to design and selection guidelines for each system component (civil works, penstock, powerhouse, generator, governor system, electrical distribution). The focus of the Global Anchor Manuals will be the documentation of the 
manufacturing process of the turbine, which includes the manufacturing drawings and CAD files, as well as supportive image and video documentation. This documentation is considered one of the practical outcomes of this research.

The study is divided into eight main chapters. The first chapter is a personal and thematic introduction to the research. The second chapter elaborates on the global crisis we are facing and can also be seen as part of my motivation for the work. In the third chapter, the modern paradigm is illustrated in relation to how the global crisis might be interconnected with the values of a modern economy. Chapter four elaborates on the concept, idea and origins of open source and open source hardware from different perspectives. Particularly, the core ideas of open source hardware and its post-capitalist approach are analyzed in this chapter. This chapter also presents examples of OSH projects, compares the two approaches and describes societal tendencies towards openness and collaboration. Chapter four also elaborates on characteristics of open source projects and compares them with living systems in nature. Furthermore the link to rural electrification is developed. Chapter five focuses on the current situation, of global remote area electrification. This section also offers an outlook on the enormous deficits, as well as the potential, of rural electrification, and deals briefly with possible renewable energy solutions for off-grid electrification. This chapter's perspective will be more technical and compares the physical concepts of transforming energy from a feasibility perspective to an applicable approach to "do-it-yourself". Chapter six highlights the technical differences of different turbine types and benchmarks them under the perspective of open source hardware. Chapter six details the feasibility study of open source remote area electrification solutions. It considers technical, economic, legal, organizational and scheduling aspects, and provides a strategy or roadmap of how to start such an endeavor. This section offers fundamental analysis and results for practical 
application. In chapter seven, a hydropower turbine is designed and presented. This part accompanies also the practical work of hydraulic design, mechanical engineering, product design, prototype manufacturing and the setup of the NGO Global Anchor, a platform for open source hydropower for remote area electrification. The thesis closes with a synthesis of the research project, and offers an outlook toward the future, with recommendations for further practical application and next steps.

Neither the methodology, nor the topic itself, is unique or new. Hydroelectric power has been used now for several hundreds of years. Similarly, the idea and approach of open source is a young development in human history, but also not new. There is already plenty of research on open source. The uniqueness of this research is the combination of the two topics; the open source approach and remote area electrification. The multidisciplinary perspective makes the research both meaningful and practically applicable, through an open source licensed product for rural electrification. I'll close this introduction with the words of Fritjof Capra:

None of its elements is really original, and several of them maybe we presented in somewhat simplistic fashion. But the ways in which the various parts are integrated into the whole are more important than the parts themselves. The interconnections and interdependencies between the numerous concepts represent the essence of my own contribution. The resulting whole, I hope, will be more than the sum of its parts (Capra, 1982: xix). 


\section{Facing the Fire - Assessment of the Global Crisis}

For the first time in history the physical survival of the human race depends on a radical change of the human heart. -- Erich Fromm (1976: 8)

To some extent my motivation for this research is derived from the global challenges we are confronted with - the basis for this chapter as an overview on the current global situation we face. What does our future look like, and how do we, as humans, want to live on this planet? With new communication technologies and increasingly sophisticated everyday technologies, life seems to be easier, at least where physical labor is concerned. As a result, we tend to go to the gym to maintain our physique. In other arenas of human life, we are permanently available, globally connected, online and multi-tasking. It seems that time goes faster and faster, and we do not have even a little moment to just hang out and let go. Every minute of time has to be filled efficiently. We don't even have time to pause and reflect on what is happening around us and within us. As Otto Scharmer puts it:

Frontline practitioners - managers, teachers, nurses, physicians, laborers, mayors, entrepreneurs, farmers, and business and government leaders - share a sense of the current reality. They can feel the heat of an ever-increasing workload and pressure to do even more. Many describe this as running on a treadmill or spinning in a hamster wheel (Scharmer, 2009: 2).

But the current global reality does not seem to be only spinning in a hamster wheel. It seems to be a crisis, touching every aspect of life. It is a crisis of meaning and values: "The crisis of our time isn't just a crisis of a single leader, organization, country, or conflict. The crisis of our time reveals the dying of an old social structure and way of thinking, an old way of institutionalizing and enacting collective social forms" (Scharmer, 2009: 2). It is a crisis of 
intention and we seem meaninglessly disconnected from life. Furthermore, for the first time in history, it is a global crisis affecting all human beings. Climate change and environmental pollution do not stop at a country's border. Climate change and pollution are global; however, there are regions that are more affected by rising sea levels than others, and there are cities that are more affected by air pollution and smog than others. Almost 35 years ago, Fritjof Capra described this scenario as follows:

It is a complex, multi-dimensional crisis whose facets touch every aspect of our lives our health and livelihood, the quality of our environment and our social relationships, our economy, technology, and politics. It is a crisis of intellectual, moral, and spiritual dimensions; a crisis of scale and urgency unprecedented in the recorded human history. For the first time we have to face the very real threat of extinction of the human race and all life on this planet (Capra, 1982: 1).

This statement describes the current situation very well, although it was formulated many years ago. The current crisis seems ungraspable and complex, and "across the board, we collectively create outcomes (and side effects) that nobody wants. Yet the key decisionmakers do not feel capable of redirecting this course of events in any significant way. They feel just as trapped as the rest of us in what often seems to be a race to the bottom" (Scharmer, 2009: 3). Forty years ago, Erich Fromm, among others, described in his book, To Have or to Be?, our disconnection to nature and our belief in ultimate technological development:

With industrial progress, from the substitution of mechanical and then nuclear energy for animal and human energy to the substitution of the computer for the human mind, we could feel that we were on our way to unlimited production and, hence, unlimited consumption; that technique makes us omnipotent; that science makes us omniscient. We were on our way to becoming gods, supreme beings who could create a second world, using the natural world only as building blocks for our new creation (Fromm, 1976: 1). 
But where is all this technological development leading us? It seems that we miss out on meaning. Where is pleasure and fulfillment, apart from materialistic growth? What is the intention of technological development? It seems that the main intention is economic growth and increasing efficiency in production and distribution: "Our obsession with economic growth and the value system underlying it has created a physical and mental environment in which life has become extremely unhealthy" (Capra, 1982: 264). We are living on a finite planet with a model of infinite growth. But where is unlimited growth in a limited system leading us? In 500 B.C. Han Fei Tzu stated: "People at present think that five sons are not too many and each son has five sons also, and before the death of the grandfather there are already 25 descendants. Therefore people are more and wealth is less; they work hard and receive little” (Meadows et al., 1972: 25).

Exponential growth in a finite system is only possible for a very short time. But still, our modern way of life is driven by the goal of growth - growth in all aspects. And technological development is driven by the industrial increase of efficiency of production. From an ecological perspective, this is a very doubtful motivation.

To use the words of the Dalai Lama:

Science is vitally important, but it is only one finger of the hand of humanity, and its greatest potential can be actualized only so long as we are careful to remember this. Otherwise we risk losing our sense of priorities. Humanity may end up serving the interest of scientific progress rather than the other way round. Science and technology are powerful tools, but we must decide how best to use them. What matters above all is the motivation that governs the use of science and technology, in which ideally heart and mind are united (Dalai Lama, 2005: 11).

The Cuban philosopher José Martí (Martí, 1974) used the words: "No hay monstruos mayores que aquellos en que la inteligencia está divorciada del corazón." Loosely translated: There are no monsters greater than those whose intelligence is divorced from the 
heart.

The following subchapters depict our major problems: energy, environment, climate change, economic inequality, meaning and values, followed by a conclusion on the interdependence and complexity of these challenges.

\subsection{Energy}

Energy is the basis of all activity: "Without energy, nothing moves nor transforms; and so a sustainable society can only exist based on a sustainable energy system" (Wissenz, 2015). Energy regimes shape the nature of civilizations - how they are organized, how the fruits of commerce and trade are distributed, how political power is exercised, and how social relations are conducted (Rifkin, 2011: 107).

The world's social and economic structures waste energetic resources at an increasing rate, and our current energy sources are centralized, based on extracting highly concentrated forms of energy, such as fossil fuels and nuclear energy, which in contrast to renewable energies are only found in certain regions of the world. Therefore, fossil fuels are also called "elite energies" and they require a significant military investment to secure their access and continual geopolitical management to assure their availability (Rifkin, 2011: 108). They also require centralized, top-down command and control systems and massive concentrations of capital to move them from underground to the end users.

Our current economic system and consumption-based lifestyle demands more and more energy. Everything seems to be based on growth and consumption, where energy is made available through a linear and highly centralized, top-down hierarchic structure. This modern society lifestyle is energetically not sustainable and provokes serious consequences for the 
natural environment. Even if we develop more sophisticated technologies we are not able to change the laws of physics; we are not able to create energy. There is, however, another side effect of energy use, which is independent of the fuel source. Energy consumption has to be seen in the frame of the first and second laws of thermodynamics, which state that energy cannot be created and that all energy transformation increases entropy ${ }^{2}$. This means: "Essentially all of the energy used by man must ultimately be dissipated as heat. If the energy source is something other than incident solar energy (e.g., fossil fuels or atomic energy), that heat will result in warming the atmosphere, either directly, or indirectly through radiation from water used for cooling purposes" (Meadows et al., 1972: 73).

In 1980, Jeremy Rifkin (with Ted Howard) published an entire book on thermodynamic limits and the future of humanity, titled Entropy: A New World View. In this book he explained:

The fact is, technology never creates energy; it only uses up existing available energy. The larger and more complex the technology, the more available energy it uses up. As awesome and impressive as our technology might sometimes appear, it too operates under the supreme reign of the first and second laws (of thermodynamics), just like everything else in nature. Those laws again: first, all matter-energy in the world is constant; it can be neither created nor destroyed only transformed from one state to another. Second, the transformation of energy is always from an available to a dissipated form, or from an ordered to a disordered state. Technology is the transformer - nothing more, nothing less.

Even though all of this is rather obvious, we still continue to live under the delusion that our technology is freeing us from dependence upon our environment, when nothing could be further from the truth. Life is not a closed system. Human beings, like all other living things, can only survive by exchanging with the environment. Without the constant flow-through of energy from the environment we would all perish within days. Technology makes us more dependent upon nature, even as it

\footnotetext{
${ }^{2}$ Entropy is a standard scientific measure of waste heat and disorder. Whenever activities consume useful energy, they produce entropy; the entropy of the world therefore increases steadily and irreversibly.
} 
physically and moves us further away from it; we have become more dependent as we have required increasing doses of nature's energy to sustain our cultural patterns and our personal life-styles (Rifkin, 1980: 95).

It is not only for the environment that we have to switch from subsurface energy supply (nuclear energy and fossil fuels) to unearthly energy (renewable energy from the sun) supply. Overcoming the decoupling from energy creation and energy consumption will lead to decentralized energy supply and energy autonomy. From a physical perspective, decentralized energy is only possible through renewable energy, since nonrenewable energy is not evenly distributed, whereas energy from the sun is natural, endless and free.

The transition from centralized nuclear energy and fossil fuels to decentralized renewable energy will have severe political, economic and structural implications (Fechner, 2010). Jeremy Rifkin makes a relevant argument about the Industrial Revolution, when he speaks about decentralized renewable energy supply:

If the First Industrial Revolution gave rise to dense urban cores, tenements, row housing, skyscrapers and multilevel factories, and the Second Industrial Revolution spawned flat suburban tracts and industrial parks, the Third Industrial Revolution transforms every existing building into a dual-purpose dwelling - a habitat and a micro-power plant (Rifkin, 2011: 45).

The necessary transition to decentralized energy autonomy is obviously clashing with the interest of the powerful energy industry: "This transition will be the biggest structural change of economy in the industrial era" (Fechner, 2010).

\section{Fossil Fuels:}

About 80 per cent of the energy that people use in the world today comes from burning fossil fuels (IEA Energy Statistics, 2014: 6), considered the most economic way to generate electricity. Fossil fuels contain a high percentage of carbon and include coal, oil and gas. As 
the name suggests, fossil fuels "are derivatives of plant and animal fossils that are millions of years old. These are primarily formed from the remains of the decayed plants and animals of the carboniferous era" (Conserve Energy Future, n.d.). Fossil fuels are a finite resource. A huge, and probably very serious disadvantage of fossil fuels is the environmental pollution they cause when they are burned. The combustion of fuels releases carbon dioxide, one of the primary gases responsible for global warming. The issue of global warming is covered separately in the following chapter on Nature and Environment.

Fossil fuels are mostly used for electricity generation and transport. Coal and gas are burned in huge, centralized and designed-for-continuous-operation power plants. During this electricity transformation process, burning fossil fuels produce heat energy, some of which is used to generate electricity. The thermal energy is then successively transformed into mechanical energy and then into electricity. Because the process is inefficient, much of the heat is released to the atmosphere or to the water that is used as a coolant. The efficiency of a coal power plant is around 45 percent, that of a gas-fueled power plant, up to 60 percent (Union of Electricity Industry, 2003: 7). More than 50 percent of today's primary energy supply is based on coal and gas (IEA Energy Statistics, 2014: 6).

Through the invention of the internal combustion engine, petroleum has powered the transportation of the modern world for almost 100 years. More than 30 percent of the world's primary energy consumption is based on burning oil. Unfortunately, the efficiency of the combustion engine is also rather low (around 30 percent), such that an enormous amount of heat is released into the atmosphere, as well (around 70 percent). Transport is globally increasing. For example, "ship traffic has quadrupled over the past two decades" (WWF Living Planet Report, 2014: 10). 
Fossil fuels cause 99 percent (coal and gas, 64 percent; oil, 35 percent) of today's carbon dioxide emissions (IEA Energy Statistics, 2014: 44). Yearly carbon dioxide emissions are twice as high as the natural environment is able to absorb through photosynthesis (Living Blue Planet Report, 2015: 24).

Apart from carbon dioxide emissions, there are several other chemical compositions released by fossil fuel combustion, which cause air pollution and acid rain: "Air pollution can result in asthma, chronic obstructive pulmonary disorder and lung cancer. Long-term exposure may increase respiratory infections in general population" (Conserve Energy Future, n.d.). In different cities around the world, we face the problem of smog and fine dust. Especially in the recent years, in many cities the use of cars is restricted to lessen air pollution and decrease health risks. Clean air is essential to life and health.

The production, transportation, and use of oil can also cause water pollution. Huge oil spill like Exxon Valdez ${ }^{3}$ and Deepwater Horizon ${ }^{4}$ are not the only examples of disasters that leave waterways and their surrounding shores uninhabitable for some time. Countless other oil spills not covered by the media also cause contamination of the environment and ground water.

Fossil fuels' integral role in human civilization cannot be overestimated, but their impact on the environment is drastically destroying human livelihood. Although fossil fuels are a limited resource, extracting and burning them completely would have severe implications on our atmosphere, a foundation for life on this planet.

\footnotetext{
${ }^{3}$ In 1989, off the coast of Alaska, the oil tanker Exxon Valdez ran aground and spilled over 30 million gallons of crude oil. It is considered to be one of the most devastating human-caused environmental disasters (New York Times, Retrieved from http://www.nytimes.com/topic/subject/exxon-valdez-oil-spill-1989, June 26, 2016) ${ }^{4}$ In April 2010, an explosion of the oil rig Deepwater Horizon, located in the Gulf of Mexico, caused the largest marine oil spill in history. Without any opposing force, oil began to discharge into the gulf. A governmentcommissioned panel of scientists estimated that 4.9 million barrels of oil had leaked into the gulf (Encyclopedia Britannica, Retrieved from http:/www.britannica.com/event/Deepwater-Horizon-oil-spill-of-2010, June 26, 2016)
} 
With all this information about dangerous hazards and health risks, it is hard to understand why we still hold on to fossil fuels as our primary energy source. Taking the following statement into consideration, it becomes even more unbelievable: "It has taken hundreds of millions of years for fossil fuels to form, and yet the sun provides more energy in a single year than humanity will ever extract from fossil fuels" (Wissenz, 2015).

\section{Nuclear Energy}

Nuclear energy seems to support our modern energy-demanding consumer lifestyle. However, the question about radioactive waste remains unanswered, not to mention the risk of catastrophic failure. The complex energy transformation process needs highly specialized technology, and "specialization goes hand in hand with increased complexity and centralization" (Rifkin. 1980: 92). Centralization and complexity limit access to the technology to very few people, to only a few companies, to only a few countries.

An average nuclear power plant brings in around 1.5 million Euros per day for its operating company (Fechner, 2010):

There is a lot of discussions on the trivial economic question of whether they will last for twenty, twenty-five, or thirty years. No-one discusses the humanly vital point that they cannot be dismantled and cannot be shifted but have to be left standing where they are, probably for centuries, perhaps for thousands of years, an active menace to all life, silently leaking radioactivity into air, water and soil (Schuhmacher, 1979: 112).

If we do not consider the environmental impact, nuclear energy seems to be good business for its operating companies. But by including waste management and decommissioning of the nuclear plant, nuclear energy proves to be much more expensive. The almost endless radiation of nuclear waste requires endless storage facilities. Endless waste by definition 
means endless costs. Including all the secondary costs related to nuclear energy demonstrates that it is also not sustainable from an economic perspective.

One problem of the general discussion on energy is its focus on climate change. It seems that the reduction of carbon dioxide becomes the highest priority. Without a doubt, nuclear energy does not emit carbon dioxide and fuels global warming much less. But the discussion presumes that "our energy supply would be totally intact if there were not the problem of carbon dioxide. But this is not the case at all" (Fechner, 2010).

There are serious problems with nuclear energy. Radioactive substances continually poison our environment: "The effects are not immediate but gradual, and they are accumulating to more dangerous levels all the time" (Capra, 1982: 258). Nuclear power implies the highest risk for our environment and life and far exceeds all other hazards of our technology: "The health hazards of nuclear power are of an ecological nature and operate on an extremely large scale, both in space and time" (Capra, 1982: 258). The technology of nuclear energy is truly a new dimension in human history, given the fact that we are able to create radioactive elements and not able to reduce their radioactivity once they are created: "No chemical reaction, no physical interference, only the passage of time reduces the intensity of radiation once it has been set going" (Schuhmacher, 1979: 111). Significant risks for health and life are obvious:

One pound of plutonium, if uniformly distributed, could potentially induce lung cancer in every person on earth. [...] Once created, plutonium must be isolated from the environment virtually forever, since even the tiniest amounts will contaminate it for eons to come. It is important to realize that plutonium does not simply vanish with the death of a contaminated organism. A contaminated dead animal, for example, may be eaten by another animal, or it may decay and rot away, its dust scattered by the 
winds. In any case the plutonium will remain in the environment and will continue its lethal action, on and on, from organism to organism, for half a million years (Capra, 1982: 262).

Plutonium is the most dangerous and most long-lifed radioactive byproduct. A nuclear power plant produces four hundred to five hundred pounds of plutonium a year. It is the basic raw material used for nuclear bombs (Rifkin, 1980: 108) and it remains poisonous for at least 500,000 years. No human technology can create safe containers for such an enormous life span:

It is difficult to grasp the enormous length of this time span, which far exceeds the length of time we are used to contemplating within our individual lifetimes, or within the lifetime of a society, nation or civilization. Half million years as shown on the chart below is more than 100 times longer than all of recorded history. It is the time span 50 times longer than that from the end of the ice age to the present day, and more than 10 times longer than our entire existence as humans with our present physical characteristics. This is the length of time that plutonium you must be isolated from the environment (Capra, 1982: 261).

How can we deal with nuclear waste in such a time span? The following graph seeks to depict the enormous time span we have to deal with nuclear waste.

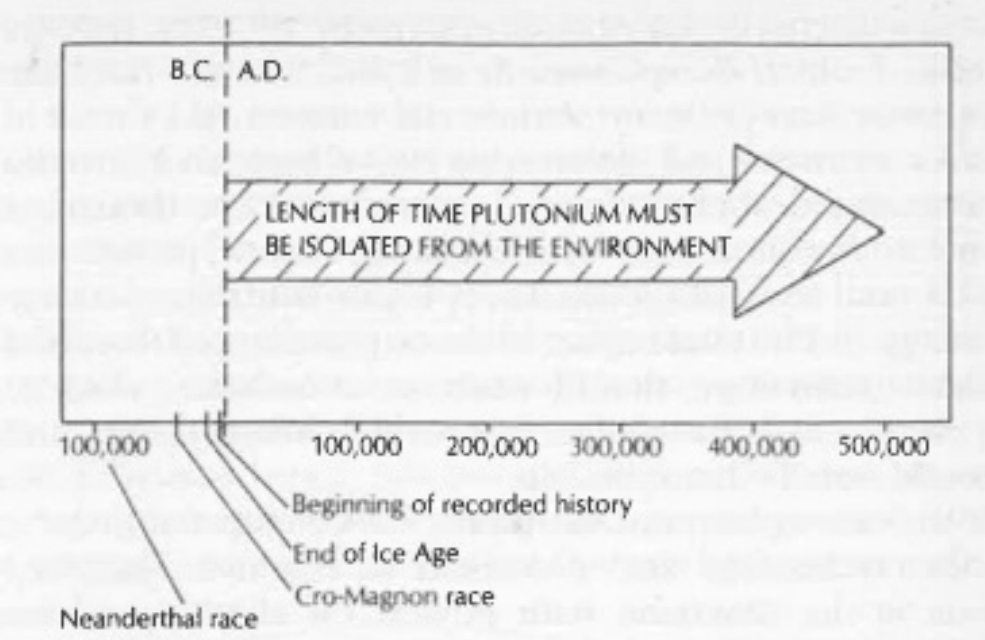

[2.1] Length of time plutonium must be isolated from the environment (Capra, 1982: 263) 
It is still an unresolvable problem: "With all the attention placed on nuclear research and development and after spending billions of dollars to erect existing plants, the scientific community, the energy companies, and the government have not yet figured out how to get rid of the radioactive waste" (Rifkin, 1980: 109). Where would a safe place be? What qualifies as a safe place?

What is a technology's value if it pollutes the environment for thousands of years while containing such high health risks for all life on Earth? What about the risk of catastrophic failure, as seen in Chernobyl and Fukushima? It is estimated that Chernobyl won't be inhabitable for at least another 20,000 years. With these two heavy nuclear accidents it is very visible that a nuclear disaster is a disaster that never ends. This chapter closes with an important question offered by Jeremy Rifkin: "What moral right do we have to leave such a deadly legacy to thousands and thousands of generations?" (Rifkin, 1980: 262).

\subsection{Nature and Environment}

Our nature and environment face several severe global problems. From water pollution to global warming and dying species, environmental issues affect every person, community and nation on this planet. We are in a state of planetary emergency, with environmental problems piling up high around us (Conserve Energy Future, n.d.). The lives of many species are threatened by pollution and climate change, and our current environmental problems require urgent attention. Population growth and our modern life-style have increased the global ecological footprint. The demand on nature is constantly increasing: "These demands include the renewable resources we consume for food, fuel and fibre, the land we build on, and the forests we need to absorb our carbon emissions" (Living Planet Report, 2014: 10). The 
Ecological Footprint in the illustration below shows that "1.5 Earths would be required to meet the demands humanity makes on nature each year” (Living Planet Report, 2014: 10).

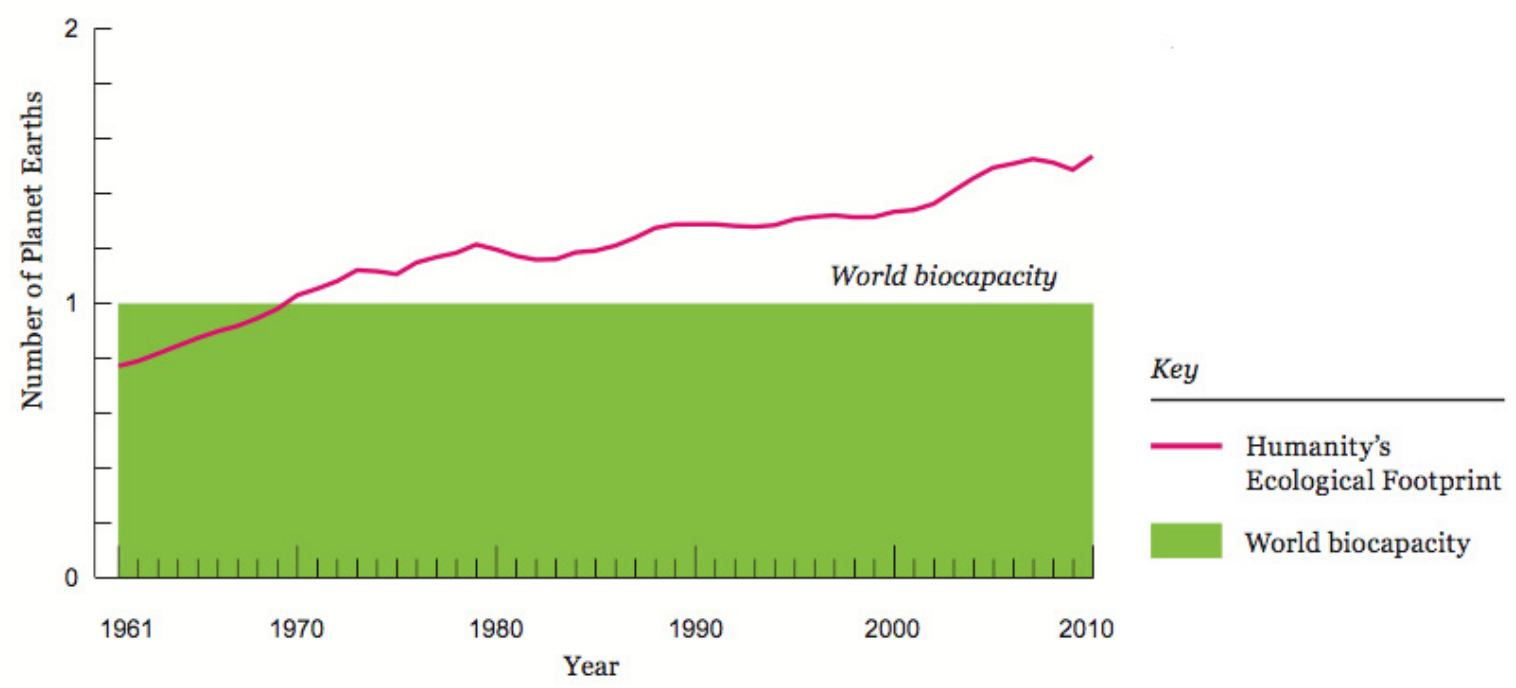

[2.2] Humanity’s Ecological Footprint (Living Planet Report, 2014: 10)

Humanity's demand has exceeded the planet's biocapacity - the amount of biologically productive land and sea area available to regenerate these resources (Living Planet Report, 2014: 10). This severe development has drastically increased in speed within the last few decades:

Over-population and industrial technology have contributed in various ways to a severe degradation of the natural environment upon which we are completely dependent for life. As a result, our health and wellbeing are seriously endangered. Our major cities are covered by blankets of choking, mustard-colored smog (Capra, 1982: $3)$.

Climate change and global warming, loss of biodiversity and the extinction of species, and pollution and deforestation are the main challenges of our time. 


\section{Climate Change and Global Warming}

Global warming has become unmistakably important. Our environment is facing the melting of ice (glaciers), rising sea levels and noticeable changes of climate. Flooding of low-lying areas and more extreme weather events are the consequences. Islands might disappear and inland agricultural zones could suffer an increase in the frequency of droughts: "In spite of alarming scientific and experiential evidence for an accelerated climate change, we, as global system, continue to operate the old way - as if nothing much has happened" (Scharmer, 2009: 3). With deforestation and burning fossil fuels (increase of carbon dioxide) we accelerate global warming. If such conditions continue, our Planet Earth will face some serious consequences in the near future.

\section{Environmental Pollution and Waste}

Pollution of air, water and soil is heavily affecting the environment. The pollution of air is a serious problem for our health. There are regions (mostly in cities) in China where air pollution is 40 times higher than the international safety standard set by the World Health Organization (Huffington Post, 2013). Because of the thick smog, buildings are barely visible, and in many regions in the world people are already getting used to wearing masks to protect their lungs from polluted air: "We can control the soft landings of space craft distant planets, but we are unable to control the polluting fumes emanating from our cars and factories" (Capra, 1982: 26). What is our intention and where are our priorities?

Water: our bodies consist mainly of water and we can't live without it. Thankfully, much of the Earth is made of water, as well. However, water pollution has widespread consequences for the entire biosphere: "Adverse alteration of water quality presently produces large scale illness and deaths, accounting for approximately 50 million deaths per year worldwide, most 
of these deaths occurring in Africa and Asia" (Hogan, 2014). Water pollution is happening through chemical or physical water pollutants, or by radioactive substances: "Locally, waste heat or 'thermal pollution' in streams causes disruption in the balance of aquatic life" (Meadows et al., 1972: 73). The most visible pollution is the physical pollution of plastic waste in our oceans. Eight million tons of plastic waste is dumped in the ocean each year (Living Blue Planet Report, 2015: 24):

Plastics are now one of the most common pollutants of ocean waters worldwide. Pushed by winds, tides and currents, plastic particles form with other debris into large swirling glutinous accumulation zones, known to oceanographers as gyres, which comprise as much as 40 percent of the planet's ocean surface - roughly 25 percent of the entire earth (Moore, 2014).

This degradation of water quality has a huge impact on marine life, and therefore also on the food chain. This means that the pollution of the ocean is entering the oceanic ecosystems, invariably polluting our food: "Clean drinking water is becoming a rare commodity. Water is becoming an economic and political issue as the human population fights for this resource. Industrial development is filling our rivers seas and oceans with toxic pollutants, which are a major threat to human health" (Conserve Energy Future, n.d.). Through industrial activity, agricultural chemicals and/or improper disposal of waste, our soils are contaminated:

We invest significant resources on our agriculture and food systems only to create non-sustainable mass production of low-quality junk food that pollutes both our bodies and our environment, resulting in topsoil degradation of a territory as large as India (the equivalent of 21 per cent of the present arable land in the world) (Scharmer, 2009: 2).

To use the words of Jeremy Rifkin: "Each day we experience the truth that biologists have long known: an organism cannot long survive in the medium of its own waste" (Rifkin, 1980: 204). 


\section{Deforestation}

Deforestation is clearing Earth's forests on a massive scale, often resulting in damage to the quality of the land: "Forests cover 30 per cent of the planet's land, and provide vital protection from sandstorms and flooding as well as the substantive natural habitat for wildlife" (Deforestation, 2015). Forests play a critical role in mitigating against global warming because they act as a carbon sink - soaking up carbon dioxide that would otherwise be free in the atmosphere and contribute to ongoing changes in climate patterns (Threat Deforestation, 2015). Forests provide vital oxygen and prevent soil erosion.

Forests are cut down for many reasons, but most of them are related to money or to people's need to provide for their families. The biggest driver of deforestation is agriculture, where land is being used for cattle and crops to meet with the increasing consumption of food. Deforestation comes in many forms, including fires, clear-cutting for agriculture, ranching and development, unsustainable logging for timber, and degradation due to climate change: "This impacts people's livelihoods and threatens a wide range of plant and animal species. Some 46-58 thousand square miles of forest are lost each year-equivalent to 48 football fields every minute" (Threat Deforestation, 2015). Deforestation is a global threat and we need to stop destroying our environment and start replanting trees and creating natural conservation areas: "The world's rain forests could completely vanish in a hundred years at the current rate of deforestation" (Deforestation, 2015).

\section{Loss of Biodiversity and Dying of Species}

Especially during the last century, we are witnessing the mass extinction of species and the collapse of our ecosystem: "Over $40 \%$ of the world's species are estimated to be at risk of extinction, primarily from human activities driving habitat loss, introduction of exotic species and global warming” (Smith, 2015). Our biodiversity includes all organisms, from 
microscopic bacteria to more complex plants and animals. Biodiversity plays an important role in the balance of our ecosystem. This includes "nutrients and water cycling, soil formation and retention, resistance against invasive species, pollination of plants, regulation of climate, as well as pest and pollution control" (Green Facts, n.d.). Energy and food security, access to clean water, and vulnerability to natural disasters are directly connected to biodiversity.

Numerous factors, such as land use, climate change, invasive species overexploitation and pollution, are combining to make species disappear much faster than before. Human activity is the primary factor leading to the extinction of species and habitats, as well as to the loss of biodiversity: "The No. 1 issue is habitat loss. Species are finding no place to live as more places are built up and altered by humans. Equally, the climate has a direct influence on the state of biodiversity. Estimates predict the loss of up to $30 \%$ of species by 2050 if climate change continues at its current pace" (Gabriel, 2007). The following graph depicts the loss of biodiversity:

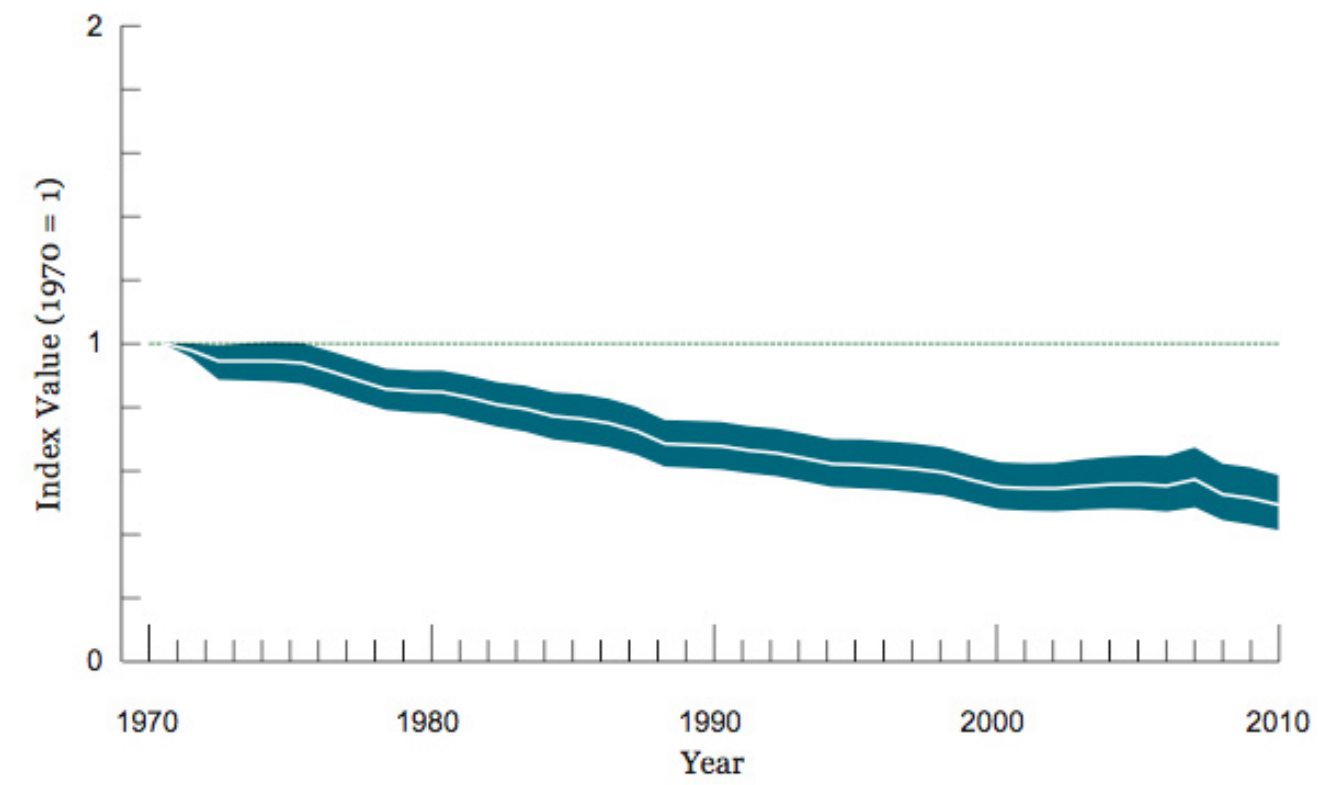

[2.3] Global Living Planet Index (Living Planet Report, 2014: 9) 
The Living Planet Index (LPI) ${ }^{5}$, published by the World Wilde Fund for Nature (WWF), indicates that there has been a decline of 52 percent between the years 1970 and 2010, which suggests that, on average, the population of vertebrate species has been reduced by half in the span of 40 years' time (Living Planet Report, 2014: 9).

From dolphins to coral, life in our oceans is suffering tremendously. The following graph 2.4 depicts the global trends in the state of marine fish stock. It is clearly visible that the world's fish stock is under pressure, "with 29 percent classified as overfished and a further 61 percent as fully exploited" (Living Planet Report, 2014: 26).

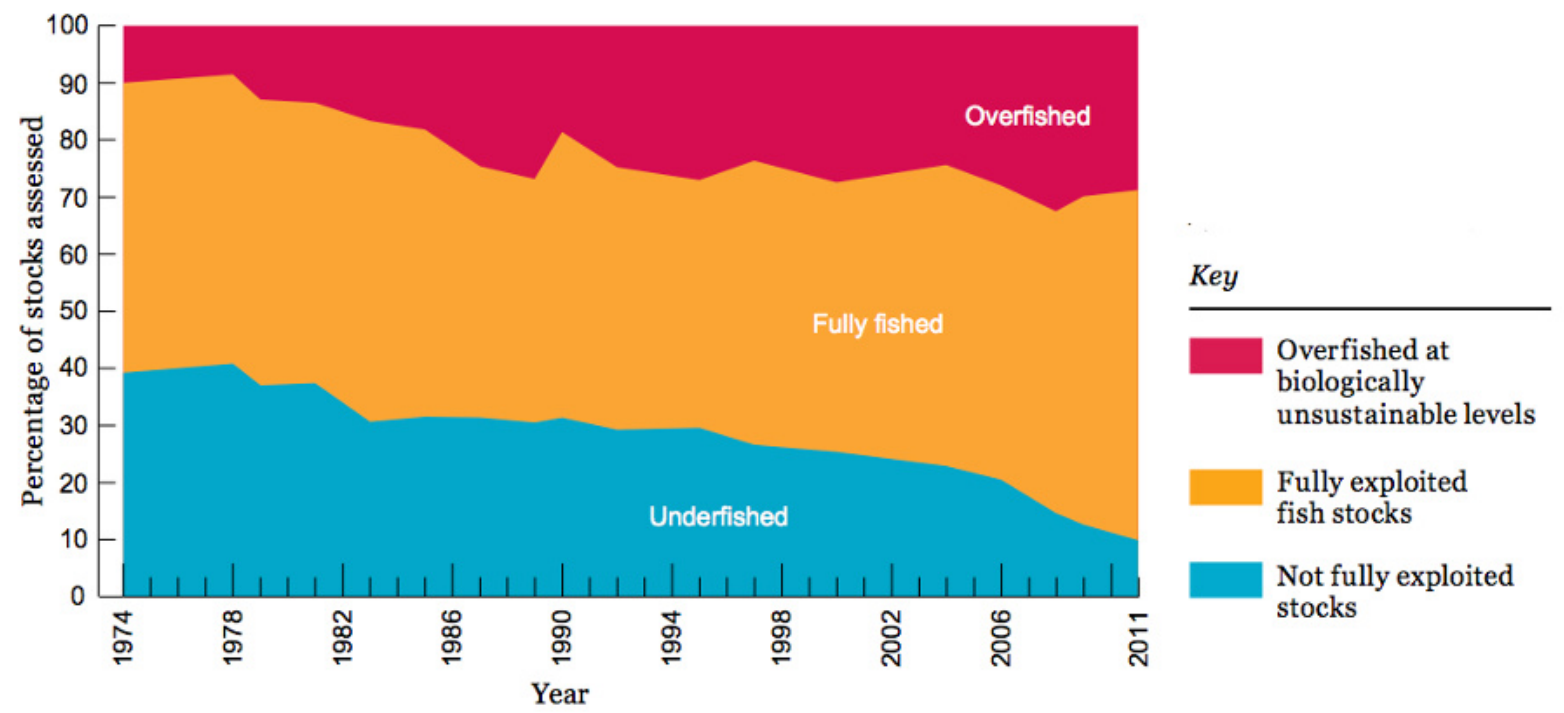

[2.4] Global Trends in the State of Marine Fish Stock 1974 - 2011 (Living Blue Planet Report, 2015: 28)

Similarly, according to the Living Blue Planet Report (2015), there has been an increase in the global average per-capita fish consumption from $9.9 \mathrm{~kg}$ in the $1960 \mathrm{~s}$ to $19.2 \mathrm{~kg}$ in 2012 . (Living Blue Planet Report, 2015: 24). The global subsidies for fishing are estimated to be US\$ 35 billion per year (Living Blue Planet Report, 2015: 26). This fuels, among other factors, a "race to fish" with severe impacts on marine life and people: "Overfishing not only

\footnotetext{
${ }^{5}$ The Living Planet Index (LPI) is a measure of the state of the world's biological diversity based on population trends of vertebrate species from terrestrial, freshwater and marine habitats.
} 
affects the balance and interaction of life in the ocean, but also the social and economic wellbeing of the coastal communities that depend on fish for their way of life" (Living Blue Planet Report, 2015: 25).

Our ecosystem is in severe danger, and it seems we are not aware of it: "As the world is ruled from towns where men are cut off from any form of life other than human, the feeling of belonging to an ecosystem is not revived" (Schumacher, 1979: 44).

\subsection{Economic Inequality}

Scharmer (2009:2) writes: "One of the primary issues is and remains that our current global system works for only a relatively small, elite minority of us, while in many parts of the world it doesn't work at all for the vast majority of the population." This is quite visible if we look at the distribution of wealth, which is very unevenly distributed. According to World Bank statistics, the global Gross Domestic Product (GDP) amounts to almost 78 trillion US\$ dollars in 2014 (World Bank, 2016). However, the GDP of the USA, China, Japan, Germany and the UK together equals 50 percent of the worlds GDP (39 trillion US\$). If we only take the USA and China into account, the global GDP share is more than 35 percent. The 20 countries $^{6}$ ranked with the highest GDP hold a world's GDP share of 80 percent (62 trillion US\$ dollar). What is more remarkable is that the combined GDP of the 180 lowest-ranked countries is still less than that of the USA (22 percent) (World Bank, 2016). It is very obvious that the population does not equally reflect GDP share around the world. The population of

\footnotetext{
${ }^{6}$ USA, China, Japan, Germany, UK, France, Brazil, Italy, India, Russian Federation, Canada, Australia, Korea Rep., Spain, Mexico, Indonesia, Netherlands, Turkey, Saudi Arabia, Switzerland 
the USA is less than five percent of the world's population, whereas the GDP of the USA is 22 percent of the world's GDP.

It is also important to note that GDP does not adequately represent the inequality of income among people inside a country. Wealth is extremely unevenly divided: "Nearly threequarters of the world's adults own under \$10,000 in wealth” (Inequality, 2015). Less than one percent of the world's population accounts for almost 50 percent of the world's wealth. As visible in the following graph, eight percent of the population holds 85 percent of the world's wealth. According to The Rules' website, "the richest 300 people on earth have more wealth than the poorest 3.5 billion" (The Rules, 2016).

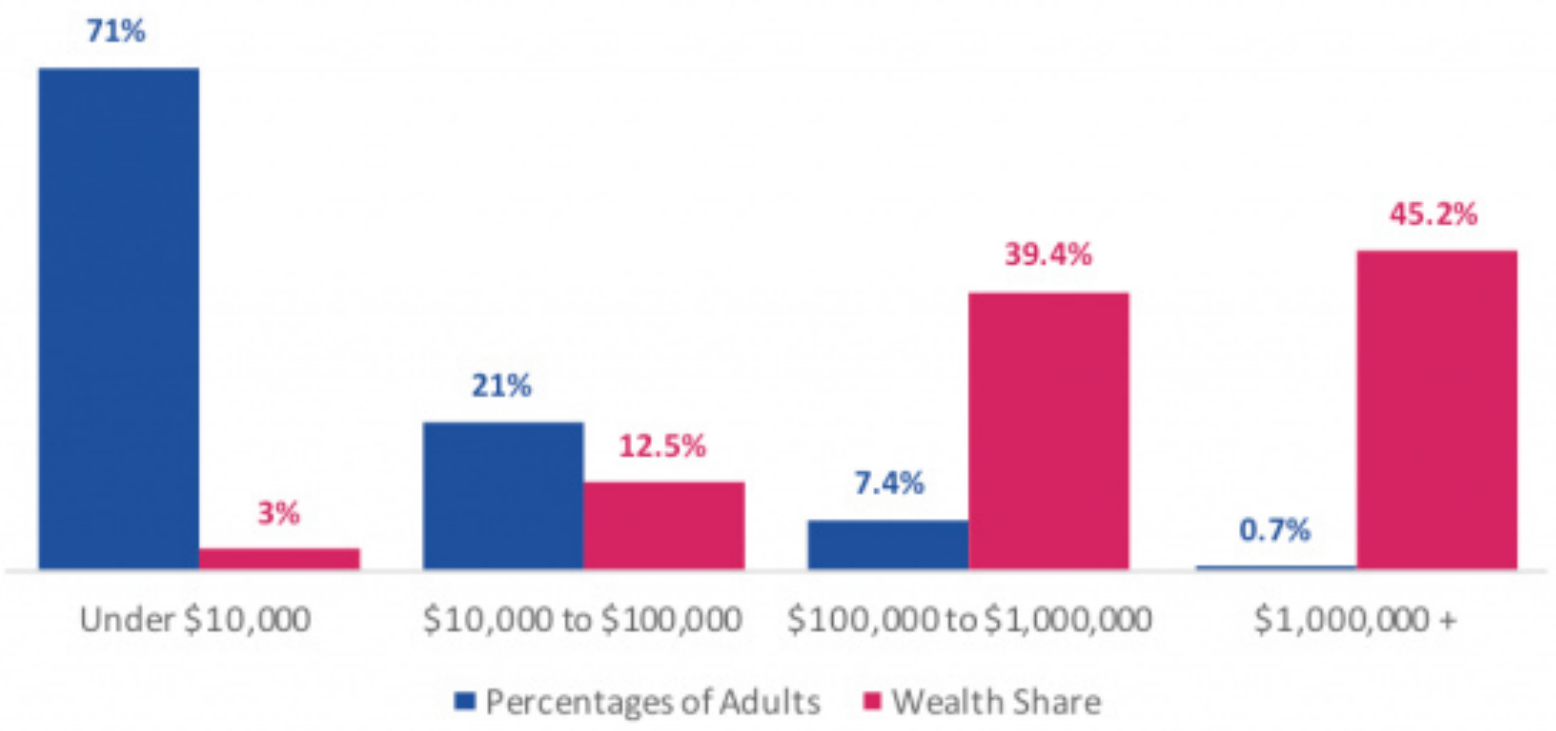

[2.5] Global Adult Population and Share of Wealth by Wealth Group 2015 (Inequality, 2015)

Where is this uneven distribution of wealth coming from, and where is it leading? What is our current global system fostering when there is so much inequality among us? Again, what is our intention? What is the intention of life? Scharmer (2009: 2) offers the following reflection: "We have created a thriving global economy that yet leaves 850 million people suffering from hunger and 3 billion people living in poverty (on less than two dollars a day). The poor of the world - about 80 per cent of mankind - live on 15 per cent of the world's total GNP.” 
As early as 1976, Fromm wrote: "Economic progress has remained restricted to rich nations, and the gap between rich and poor nations has ever widened" (Fromm, 1976: 2). This statement is still very real, now forty years later. Money keeps hijacking the political process. Further, Rifkin (1980: 195) explains the danger of such a concentration of wealth and power in very few hands: "History shows that whenever a societies energy (wealth) becomes so concentrated in the hands of a few individuals or institutions that the rest of the society suffers energy deprivation so great as to imperil their own survival, the society either crumbles or moves to revolution or both. While nature relies on self-regulating biological laws to restore balance, society must rely on agreed-upon principles of economic justice to achieve the same ends."

\subsection{Meaning and Values}

We have reduced reality to that which can be measured, quantified, and tested. We have denied the qualitative, to spiritual, the metaphysical. We have entered into a pervasive dualism - our minds separated from our bodies, our bodies divorced from the surrounding world. We have gloried in the concepts of material progress, efficiency, and specialization above all other values. In the process, we have destroyed family, community and tradition.

-- Jeremy Rifkin (1980: 204)

We focus on quantification and measurement. Our values are materialistic growth and personal fulfillment, which is often also archived through material growth or possession. Growth has become the most important value in the neoliberal capitalist worldview. And this worldview is spreading all over the world. Especially now that new communication technologies and globalization are increasingly omnipresent, money is all that counts. This also leads to more individualism, isolation and the breakdown of social structures: "The resulting loss of culture and structure leads to eruptions of violence, hate, terrorism and civil war, along with partly self-inflicted natural catastrophes in both the southern and the 
northern hemispheres" (Scharmer, 2009: 4).

In the following chapters on paradigms and open source, in particular, this thesis will deepen into humanity's crisis of meaning. For now, the following quote describes our current situation very well:

On his 1977 lecture tour of the United States, E. F. Schumacher noted: 'The most urgent need our time is and remains the need for metaphysical reconstruction, a supreme effort to bring clarity into our deepest convictions with regards to the questions what is man? Where does he come from? and What is the purpose of life?' These are the big questions of human existence, questions which have absorbed people for thousands of years. Today, in our nine-to-five existence, they are not much discussed and are, in fact, dismissed as being prescientific because they do not fit into the neat little standardized explanations of the world offered us by the Newtonian world view. Nonetheless, the big questions of the past are destined to reemerge in the low entropy-world that awaits us. For a low-entropy energy environment provides a completely different orientation to the goals of humanity. The governing ethical principle of low-entropy world view is to minimize energy flow. Excessive material wealth is recognized as irreversible diminution of the world's precious resources. In the low-entropy society 'less is more' becomes not a throw-away phrase but a truth of the highest magnitude. A low-entropy society deemphasizes material consumption. Frugality becomes the watchword. Human needs are met, but whimsical, selfindulgent desires - the kind of pandered to in every shopping center in the country are not. The traditional wisdom, as embodied in all the great world religions, has long taught that the ultimate purpose of human life is not the satisfaction of all material desires, but rather the experience of liberation that comes from becoming one with them metaphysical unity of the universe. The goal is to find 'the truth that will set us free'; to find out who we really are (Rifkin, 1980: 205).

Obviously, these questions do not seem important in a material growth-based society. Furthermore: "Unwarranted consumption, possessions and a general attachment to material things have been discouraged by all the great religious teachers in the past" (Rifkin, 1980: 206). 


\subsection{Conclusion}

Something is wrong with how the world is right now. Through our centralized, fossil fuel based energy sources we pollute our environment and speed up climate change. The uneven distribution of natural energy resources leads to violence and war. Apart from the enormous risk of catastrophic failure, nuclear energy has to be protected by military and police from terrorist attacks. For centuries, people have regarded nature as an inexhaustible source of raw materials and a convenient dumping ground, too vast to be affected by anything we do. In combination with technical development, we have reached a point where our natural environment is seriously in danger. No matter where we turn, every aspect of life seems to be seriously affected. Our seas are full of waste, affecting our food chain. Species are dying at a very high rate because of the reduction of living space and climate change. We use chemical fertilizers to make our monocultures grow more efficiently, and in the process are killing off the bees necessary for the pollination of plants and our very survival. We cut trees and decrease our forests, the "lungs of the world". We produce nuclear waste and have no clue how and where to store it for the next 500,000 years. The list could go on and on, and it is scary to contemplate when we look at our current problems and challenges.

And the question is: What for? Fromm (1976: 2) argues that "the achievement of wealth and comfort for all was supposed to result in unrestricted happiness for all." Under the assumption that wealth leads to happiness, we are not even reaching that goal. Because of the extremely uneven distribution of wealth, the majority of humanity lives in poverty.

We do not recognize the limitations of the dualistic, mechanistic world view. The current crisis seems to be a crisis of perception. In reality, the threats are all connected, yet we continue to deal with them separately in a piecemeal fashion: "What is most evident is that all these problems are interconnected that none of them can be solved in isolation. They are 
systemic problems, interconnected and interdependent" (Capra, 2015). This interdependence of all things is visible in many of the world's contemporary challenges:

Climate change cannot be addressed in isolation from the wealth-hoarding of capitalism that has made the world so unequal that a mere 62 individuals have the same aggregate wealth as 3.7 billion. Terrorism cannot be tackled in the absence of deep inquiries about what happens when money is treated as more sacred than life or spiritual tradition. Political corruption cannot be wrangled in without taking account of the commercialization of elections that treats each candidate like yet-anotherproduct to be bought and sold in the marketplace of ideas (Brewer, 2016).

Humanity has lived on this planet for thousands of years in different forms social organization. For example, we lived in egalitarian forms of society, which awarded cooperation and sharing, and punished selfishness. Today, we hold onto a system that is not making us happy and threatens our environment and life on this planet: "As long as the driving feedback loops of population and industrial growth continue to generate more people and a higher resource demand per capita, the system is being pushed toward its limit-the depletion of the earth's nonrenewable resources" (Meadows et al., 1972: 68).

With this rather dark and depressing assessment of the global crisis, the next chapter elaborates on the modern value system and where it comes from. Furthermore, it elaborates on alternatives and establishes the link to the approach of open source. 


\section{Paradigms}

The major problems in the world are the result of the difference between how nature works and the way people think. -- Gregory Bateson (Bateson, N. 2011)

Scientific knowledge is evolutionary and situated in time and space. We are living in a paradigm shift from mechanistic to organic, from machine to living. This chapter elaborates on the term "paradigm" and the "paradigm shift". The Merriam-Webster dictionary defines the term paradigm as "a philosophical and theoretical framework of a scientific school or discipline within which theories, laws, and generalizations and the experiments performed in support of them are formulated" (Merriam Webster, n.d.). This framework includes basic assumptions, methodology and the way people think. A paradigm shift is the radical change of the way of thinking in any discipline or group, which provokes a fundamental change of an individual's or a society's values and behavior. The term "paradigm shift" was formulated by the American philosopher and physicist, Thomas Kuhn. He introduced the term in his book, The Structure of Scientific Revolutions, published in 1962. In this book, Kuhn argues that science does not progress via a linear, continuous path, but rather undergoes periodic paradigm shifts. According to Kuhn, a scientific revolution occurs when scientists encounter anomalies that are not explained by the current, universally accepted paradigm. With numerous significant anomalies, the current paradigm is thrown into a state of crisis from where new ideas and approaches arise and eventually form a new paradigm. These paradigm shifts open up new approaches to understanding what scientists would never have considered valid before. Kuhn (1962: 113) writes: "What a man sees depends both upon what he looks at and also upon what his previous visual-conceptual experience has taught him to see." This means that scientific truth, at any given moment, is defined by the consensus of the scientific 
community. Further, science can never rely wholly upon objectivity as the ultimate truth. German theoretical physicist, and one of the key pioneers in quantum physics, Werner Heisenberg argued: "We have to remember that what we observe is not nature in itself but nature exposed to our method of questioning” (Heisenberg, 1962: 58). Therefore, our science is very limited. Science must account for subjective perspectives, as well, since all objective conclusions are ultimately founded upon the current scientific paradigm of its scientists.

The following chapters elaborate on the current mechanistic paradigm and the rise of an organic, living paradigm, of which the open source approach may be considered a part. The chapter concludes with a comparison of the two paradigms.

\subsection{The Modern Paradigm}

There is more to human existence and to reality itself than current science can ever give us access to. -- Dalai Lama (2005: 13)

The current paradigm in which we live is based on the modern concepts of predictability and control. It can also be called a scientific concept, which means there is no mind; that everything is matter. Everything that cannot be measured practically does not exist. The strictly mathematical and logical approach to science focuses on the quantitative, objective characteristics of matter (form, number, and movement). Other characteristics like color, sound, taste, or smell are seen as subjective projections of the mind and are expulsed from science (Dietrich, 2008: 241). The world, human beings and organizations are machines (Robert, 2011). In this context the modern paradigm is not understood as a clearly definable epoch with definite temporal demarcation of beginning and end. It is more a state of mind, for 
which several characteristics can be defined. In his lecture, "Transrational Interpretation of Peace”, at the University of Innsbruck, Norbert Koppensteiner (2010) offered the following words: "Modernity could be so grasped as the societal project characterized by Cartesian reductionism, Newtonian physics, the capitalist world-system and the nation state of Thomas Hobbes." Similarly, Francis Bacon, Nikolaus Kopernikus, Johannes Kepler, Galileo Galilei and Charles Darwin also have made their impact on the modern belief system (Dietrich, 2008: 241). The following sub-chapters depict the origin of this worldview and how it affects different aspects of daily life.

\section{Architects of the Modern World}

The modern paradigm arose around 300 to 400 years ago with the ideas of René Descartes, Isaak Newton, Thomas Hobbes and Francis Bacon. After 300 years, we are still living off of their ideas (Rifkin, 1980: 19). As already mentioned, knowledge is situated in time and space. The brutal environment of the "Thirty Years War" could be seen as a key factor for Thomas Hobbes, who lived in that time. His principle motivation for action is fear (Koppensteiner, 2010). His famous statement "Homo Homini Lupes est!"7 expresses his worldview and the basis of his ideas. He sees life as "nasty, brutish and short" (Hobbes 1651: 76). But this is unreasonable and against the interest of everybody. Therefore, he argues for a strong state as means of order within the society. According to this line of thought, the defence of internal welfare against others could only be reached by protectionism (mercantilism) and rearmament (Koppensteiner, 2010).

With his expression, "Cogito Ergo Sum", the work of René Descartes could be seen as the beginning of the modern philosophy of the subject. Descartes is attributed with the popularization of the machine metaphor and the split between mind and body, subject and

\footnotetext{
${ }^{7}$ Man is wolf to man.

${ }^{8}$ I think, therefore I am.
} 
object. He saw the body as a biological machine and the mind as something apart from the body. The biological machine can be reduced to its parts. This approach is quite visible in modern "industrial" medicine, where damaged, separate, replaceable parts of the biological machine we call the body, can be replaced with new ones by surgery. We are able to exchange a heart the same way we change the engine of a car. In his worldview, the being is reduced and "becomes a function of thinking" (Koppensteiner, 2010). The subject is the thinking being, the observer; and everything that is observed is object. Everything else, including other people and one's own body are categorized as object. This results in the assertion that only rational thought is true. The distinction between mind and body is called Cartesian dualism. Consequently, all science is considered certain and evident knowledge: "We reject all knowledge, which is merely probable and judge that only those things should be believed which are perfectly known and about which there can be no doubts. God is no longer necessary. Scientific truth is absolute" (Koppensteiner, 2010). The basic understanding of modern science is rooted in this assumption.

At the time of Descartes, kingdoms were interested in warfare, predictability and control (Robert, 2011). Merchant-driven research was interested in how to improve and make ships safer and more efficient by bringing spices from the East, for example. Linear predictability was important and the merchant-financed research defined the research outcomes (Robert, 2011). This type of linear connectivity is still important in our time. For example, insurance companies look into the past to predict the future (Robert, 2011), which is a process based on exactly the same principle. Embedded in this same time period, Isaak Newton developed three laws of motion, which were intended to describe the motion of the planets. It turns out that these laws could be applied in a seemingly general way (Mikulecky, n.d). Newton created a mathematical theory of the world and explained physical phenomena as action and 
reaction, cause and effect. As a consequence, the universe is determined by laws, "which in principle can be understood" (Koppensteiner, 2010). This means that if and when all the variables influencing a natural phenomenon can be described exactly, then a precise prediction would be possible. The universe works with the precision of clockwork. Time is reversible and everything is predictable. This means also that what we cannot predict is what we haven't yet understood, but will in the future. The broader application of these principles is the foundation of the modern scientific method (Mikulecky, n.d.).

Furthermore, it would be remiss not to mention the contributions of John Locke and Adam Smith to the modern paradigm: "John Locke brought the workings of government and society in line with the world machine paradigm, and Adam Smith did the same with the economy" (Rifkin, 1980: 23). Smith can be seen as the father of modern economics and laid the foundations of classical free-market economic theory: "Smith explicitly removes any notion of morality from economics, just as Locke had done with social relations" (Rifkin, 1980: 27).

These ideas, taken together, resulted in a worldview that excludes aesthetics, morals, values, sensations, feelings, and awareness (Dietrich, 2008: 241) from the scientific method. Spirit and soul have no space in science. And science, as a process of paradigm and a result of worldview, is perceived as universal truth.

Through colonization, this worldview and its accompanying values have spread throughout the world. Most of modern science and technology is a result of the Newtonian paradigm and Descartes reductionism. This worldview is visible in organizational structures, economics, science, medicine and almost all aspects of life. The following subchapters elaborate on the modern paradigm in certain aspects of life. 


\section{Paradigms}

\section{Science and Technology}

Modern approaches recognize as real only that which can be empirically described - they focus on the outer and secular aspects of existence (Koppensteiner, 2010). The universe is made out of material objects, which can be analyzed, understood and improved. Science and research is often financed and directed by private companies and governmental grants. This leads to technologies that improve humanity's living standards in many ways. The development of transport technology, for example, has improved rapidly within the last century; communication reached a new global connectedness in only a few decades. Technological changes are being introduced at an extremely high rate. However, these technological improvements are very linear and mechanistic, not to mention their impact on the environment. This begs the question: what is this technology for? Schumacher questions the benefit of technological improvements, as follows: "Scientific or technological 'solutions', which poison the environment or degrade the social structure and the man himself, are of no benefit, no matter how brilliantly conceived or how great their superficial attraction" (Schumacher, 1973: 20).

It is obvious that we cannot live without science and technology, and there is no doubt that there are many benefits from technological development. But the scientific direction of development is important. Alfred Einstein himself said, "almost all scientists are economically completely dependent and the number of scientists who possess a sense of social responsibility, is so small" (cited in Schumacher, 1973: 116). Science, in this worldview, is driven by increasing the profit of private companies, where a common human interest is secondary. It is no secret that physical products are engineered so that specific parts break after the designated time, with the intention to sell spare parts and make consumers buy new products. The oil and pharmaceutical industries, for example, have enormous influence on the political framework. 
Another aspect worth mentioning is the increasing complexity and specialization of technology. Today, one person alone is unable to build a car. Building a car requires many specialists who provide a little piece of the whole product. This has severe disadvantages for society and is probably not a healthy development for human society, as Rifkin explains from an energy transition perspective:

We have come to the point where each of us knows more and more about last less, until as a society we all know almost everything about nothing. Overspecialization, say the biologists, is one of the most important contributing factors in a species' becoming extinct. When a species becomes over specialized in the particular type of ecosystem, it is usually unable to adapt to a change in environment. It does not contain the flexibility and diversification to enable it to make the transition. The same holds true with human society. Today we have become so overly specialized and adopted to the existing energy environment that we have lost much of the flexibility required to make a transition into a radically new energy mode (Rifkin, 1980: 93).

With this way of life, we aim to have experts in every field. As a result, we transfer the power to someone else we trust and stay powerless on our own. But these "experts" are struggling with their fields of expertise - today even moreso than thirty years ago, when Fritjof Capra (1982: 5) wrote:

It is a striking sign of our time that the people are supposed to be experts in various fields can no longer deal with the urgent problems that have arisen in their area of expertise. Economists are unable to understand inflation, oncologists are totally confused about the causes of cancer, psychiatrists are mystified by schitzophrenia, police are helpless in the face of rising crime, and the list goes on.

Technological development is mainly driven by the mechanistic worldview. Subjectivity and research that goes beyond measurement and proof are not popular, nor are they supported, despite the reality that for human development they might be as important as our current technology and science. 


\section{Economy, Growth and Energy}

Our current economic system is based on consumption and growth. The typical lifecycle of a product is: buy, consume, throw away; a process that now happens in a shorter and shorter timeframe, supported by governments and media. A mobile phone is replaced after two years because the communications company is offering a new one for free. The life span of a car nowadays is much less than it was 20 or 30 years ago. It is obviously impossible in a finite system to provide an increasing population with ever-increasing "materialistic wealth". What will be the environmental impact of air pollution by having one billion Chinese driving cars? Not to mention the environmental impact of the energy consumption for the production of one billion vehicles. Ernst Schumacher (1979: 17) describes the desire to unlimited material wealth in a limited system as follows: "An attitude to life which seeks fulfillment in the single minded pursuit of wealth - in short, materialism - does not fit into this world, because it contains within itself no limiting principle, while the environment in which it is placed is strictly limited." On top of that, it seems that materialistic growth has replaced personal fulfillment. Erich Fromm (1976: 6) observed this development forty years ago and put it into the following words:

The development of this economic system was no longer determined by the question: What is good for Man? but the question: What is good for the growth of the system? One tried to hide the sharpness of this conflict by making the assumption that what was good for the growth of the system (or even for a single big corporation) was also good for people. This construction was bolstered by an auxiliary construction: that the very qualities that the system required of human beings - egotism, selfishness and greed - were innate in human nature; hence, not only the system but human nature itself fostered them. Societies in which egotism, selfishness, and greed - did not exist were supposed to be primitive, their inhabitants childlike. People refused to recognize that these traits were not natural drives that caused industrial society to exist, but that they were the products of social circumstances. 
An economy based on growth for an increasing population will ultimately reach a point where resources get very limited. What is it that we are actually growing when we grow the economy? The spirit of growth is blinding the fact that it is just a matter of time until natural resources reach their limits and "nature will fight back against human rapaciousness" (Fromm, 1976: 6). Moreover, human relations are changed by increasingly scarce resources, as Schumacher (1979: 45) describes:

As the world's resources of nonrenewable fuels - coal, oil and gas - are exceedingly unevenly distributed over the globe and undoubtedly limited in quantity, it is clear that their exploitation at an ever increasing rate is an act of violence against nature which must almost inevitably lead to violence between men.

Energetic predictability is very important in the current mechanistic worldview, primarily for the economy. Within this worldview, it is purely logical that the energy supply in such a system is provided in a hierarchical and centralized way. The current system is provoking a top-down, centralized energy supply, which makes us dependent on global companies distributing unevenly distributed resources. Our energy supply is based on fossil fuels and nuclear energy, allowing for such centralized, hierarchical distribution.

\section{Nature and the Environment}

In previous chapters we have explored the challenges associated with the current state of nature. To a large extent, these challenges can be seen as a result of our behavior and alienation from nature. John Locke declared: "The negation of nature is the way toward happiness'. People must become 'effectively emancipated from the bonds of nature" (Rifkin, 1980: 24). In a related sense, Bacon's empirical approach helped to clearly separate science from philosophy. He established an approach of using data collection to develop and test scientific theories. The proposed scientific method of Francis Bacon has influenced science a 
great deal, especially regarding the relationship of men to nature. Bacon understood nature as female, and wanted to treat nature with his empirical scientific method like the women were being treated at the witch trials during that time (Dietrich, 2008: 242). According to Carolyn Merchant (1980: 169) Francis Bacon advocated that nature be "hounded in her wanderings and made into a slave" and proposed that nature's secret should be tortured from her. His anti-woman, anti-nature stance reflects his culture, but it also reveals the origin of an important aspect of science that is still evident today - that the goal of science is to dominate nature (Oakley, 1995).

Today, we live in cities and lose connection to our natural environment. The temperature outside our apartments does not matter anymore. We have artificial climates, with heat or airconditioning. Water and electricity come out of the wall. Our food comes from the supermarket. Alienation from nature is visible in every modern society. With this alienation, people's relation to nature has become deeply hostile: "We have tried to solve our existential problem by giving up the Messianic vision of harmony between humankind and nature by conquering nature, by transforming it to our own purpose until the conquest has become more and more equivalent to destruction" (Fromm, 1976: 6). Sadly, nature is just as vulnerable as nation-states protecting themselves against the interests of corporations.

\section{Modern Values and Organizational Structures}

The mechanistic worldview is visible in our value system, as well. The Darwinian approach of 'survival of the fittest' seems applicable to our modern societies. We do not share; we compete. Fromm (1976: 5) describes this phenomenon in his book, To Have or to Be?:

The pursuit of individual egoism leads to harmony and peace, growth in everyone's welfare, is equally erroneous on theoretical grounds, and again its fallacy is proven by the observable data. To be an egoist [...] means: that I want everything for myself; that 
possessing, not sharing, gives me pleasure; that I must become greedy because if my aim is having, I am more the more I have; that I must feel antagonistic toward all others; my customers whom I want to deceive, my competitors whom I want to destroy, my workers whom I want to exploit. I can never be satisfied, because there is no end to my wishes; I must be envious of those who have more and afraid of those who have less.

He argues that we will not be happy and will suffer with egoism and competition. Consumption and personal fulfillment are important for us, and we do not care what resources we exploit to satisfy our "needs". We control nature and take from her what we think we need. We fly to the moon just for fun. Our acts are based on rational interest only. We exclude emotions and feelings, which are important drivers of our actions (Robert, 2011). Most of our organizational structures are top-down with strong hierarchies, where nothing moves until there is an impulse from the outside. The precondition or necessity for movement is an order from a higher layer, the superior or boss. The motivation for action is because of money, which allows "personal fulfillment" (Robert, 2011). This is a clear linear structure and works through the principle of action and reaction, as in the functioning of Newtonian Physics.

\section{Conclusion}

In a modern understanding, the world can be explained using the scientific principles of cause and effect. We have reduced the universe to material objects; a mechanical system composed of elementary building blocks, which can be measured, analyzed, understood and improved. God is no longer necessary. Scientific truth is absolute. Our minds are separated from our bodies, our bodies divorced from the "surrounding" world. We place men at the center of the universe and "define the ultimate purpose of our existence as the satisfaction of all possible materials wants, however frivolous" (Rifkin, 1980: 94). We exclude the qualitative, the 
spiritual, the sensation and the feeling. Life in society is seen as a competitive struggle for existence (Capra, 1996: 6). We believe in the concepts of unlimited material progress, archived through efficiency and economic growth. With our values we "have destroyed family, community, tradition. We have left behind all absolutes, except for absolute faith in our ability to overcome all limits to our physical activity" (Rifkin, 1980: 94). We are exploiting and polluting nature as never before in human history, and we push aside the alarming news about the environment and climate change as if they were not even real. This paradigm has dominated our culture for several hundred years, has shaped our modern western society and values, and has significantly influenced the rest of the world.

Let us conclude this chapter with a 40-year-old quote from Jeremy Rifkin, the significance of which is still poignant today:

The more our world slips deeper into chaos, the less willing we are to identify the source of the problem. Instead, we wrap ourselves up even tighter in our technological garb, defending it against all criticism, unable to acknowledge what it is doing to the environment we live in, and even less able to acknowledge what it is doing to us. We continue to cling to the fiction that we are securely clothed and protected, even as we become more exposed and endangered by the disordered fragments of the world of our own making (Rifkin, 1980: 96). 


\subsection{A systemic, organic Paradigm based on Life}

Indeed, to some extent it has always been necessary and proper for man, in his thinking, to divide things up, if we tried to deal with the whole of reality at once, we would be swamped. However when this mode of thought is applied more broadly to man's notion of himself and the whole world in which he lives, (i.e. in his world-view) then man ceases to regard the resultant divisions as merely useful or convenient and begins to see and experience himself and this world as actually constituted of separately existing fragments. What is needed is a relativistic theory, to give up altogether the notion that the world is constituted of basic objects or building blocks. Rather one has to view the world in terms of universal flux of events and processes. -- David Bohm (1980)

The "other" paradigm we are entering is based on unpredictability, non-linearity and life. It can be seen as organic, holistic and based on a new scientific understanding of life at all levels - organisms, social systems and ecosystems (Robert, 2011). The rising paradigm includes what has been excluded in the mechanistic-dualistic worldview. The spiritual, the emotional, the qualitative and feelings have an important part in this new paradigm. At the same time, it does not reject the modern paradigm but acknowledges its validity in a limited frame. The new paradigm "is based on a new perception of reality that has profound implications not only for science and philosophy, but also for business, politics, health care, education, and everyday life" (Carpa, 1996: 3). This scientific revolution started with the emergence of new sciences: quantum physics, thermodynamics, complex thinking, chaos theory, biosciences, ecology, gender studies, etc. (Robert, 2011). At the beginning of the last century, it became obvious that the modern, mechanistic approach is not sufficient to describe the world. It is a shift from Descartes and Newton to Einstein; a shift from the machine to patterns and living beings.

The mathematician Copernicus placed the sun at the center of the universe, which triggered the Copernican revolution and made the existing worldview at the time (the Earth is flat) 
obsolete. We all move around the sun, as the fixed point of the universe. The fact that there is only one point of reference in the universe was valid for almost 400 years. With Albert Einstein, a new paradigm began. Einstein came up with the idea that there is not only one point of reverence in the universe, but that there are many; and on top of that, they move (Robert, 2011).

Einstein, with the theory of relativity, was one of the first scientists at the forefront of what has been called "non-mainstream thinking", which began around a hundred years ago. Nonmainstream thinking was first in the field of physics, but gradually other areas were affected: biology, medicine, psychology and economics. All these disciplines deal with life in one way or another; "with living biological or social systems" (Capra, 2002: xvi). The following pages depict several streams (systems thinking, cybernetics and deep ecology) of this new understanding of reality, in which life itself is situated at the center.

\section{System Thinking}

The main characteristics of systems thinking emerged simultaneously in several disciplines during the first half of the last century, especially during the 1920s (Capra, 1996: 17). With systems thinking, a completely new form of thinking emerged; away from linear cause-andeffect toward connectedness, relationship and context (Robert, 2011). From the perspective of systems thinking, everything is connected with everything. We live in and are made of systems, embedded in systems. The approach of systems thinking is fundamentally different from classical forms of analysis. From a traditionally "modern" perspective, an analysis tries to reduce the studied object into separate individual parts (Aronson, 1996). Systems thinking is a perspective of seeing systems as a whole rather than as collection of parts: "This means that instead of isolating smaller and smaller parts of the system being studied, systems 
thinking works by expanding its view to take into account larger and larger numbers of interactions as an issue is being studied" (Aronson, 1996). This approach is the opposite of reductionism and also tries to integrate the excluded "spirit, soul and feelings":

Systems theory looks at the world in terms of interrelatedness and interdependence of all phenomena, and in this framework an integrated whole whose properties cannot be reduced to those of its parts is called a system. Living organisms, societies, and ecosystems are all systems (Capra, 1982: 26).

According to systems theory, the essential properties of an organism are properties of the whole. The so-called whole of investigation is a web of interconnectedness, which is also a key part in elicitive conflict transformation (Dietrich, 2010). Each part of the whole has different properties, and the properties of the whole arise from the interactions and relationships of its parts. Similarly, Capra (1996: 29) explains that "these properties are destroyed when the system is dissected, either physically or theoretically, into isolated elements." Systems thinking looks at the larger context. With this approach, the results of investigation are strikingly different from those generated by classical scientific forms of analysis. Systems thinking becomes very important in the context of life. As we will see later, all life consists of living systems. Albrecht Mahr, (1996, as cited in Mayr, 2010: 23) stated: "as soon as there is life, there are social systems to which we are bonded to by nature." In systems theory, the essence of life is self-organization.

\section{Cybernetics}

Cybernetics is about having a goal and taking action to reach that goal. The word Cybernetics comes from the Greek word meaning "the art of steering" (Pangaro, 2012). Cybernetics is a trans disciplinary approach for exploring regulatory systems, their structures, constraints and possibilities. Anthropologist Gregory Bateson noted that whereas previous sciences dealt 
with matter and energy, the new science of cybernetics focuses on form and pattern. Cybernetics focuses on the behavior of things and not on their material composition. The mathematician and philosopher Norbert Wiener is known as the originator of cybernetics. In 1948, he published the book, Cybernetics: Control and Communication in the Animal and the Machine, which offered a definition of cybernetics. In this book, he describes regulatory systems with goals, feedback loops, action, stability and regulation. His works on cybernetics has had a significant influence on engineering, biology, computer science, systems control and psychology, because many systems in the living, social and technological world can be understood in this way. These regulatory characteristics are evident in all intelligent systems, since:

all intelligent systems have this property of trying, acting, seeing the difference, changing, acting, seeing, sensing. This loop of acting, sensing, comparing to goal, acting sensing, comparing, is fundamental. A system cannot be intelligent unless it has those properties, because otherwise it goes and it succeeds or fails and who knows? But this way there is the self-correction all the time. So, a technological system, at least these interacting systems we are talking about, have these properties of self-correcting (Pangaro, 2012).

Here is an example: A self-correcting system could be a person navigating a ship. His destination is visible and he tries to navigate to it. By doing so he is acting on the steering wheel, which changes the direction of the boat. With a changed and new direction, he is now sensing his surroundings again and perhaps correcting the direction again, as well (Wiener, 1948: 7). Another example is a heating or air-conditioning system in a room. It is constantly measuring the temperature in the room. If the temperature is lower than the desired temperature, the system acts and the heating goes on. When the temperature is above the desired temperature, the system acts again and shuts down the heat (Ashby, 1956: 198). 
In the first example, the actions of the person navigating the ship will move it closer to his destination or ultimate goal. The same applies for the temperature regulation system. The measuring and permanent information flow is defined as a feedback loop, which can be positive or negative.

Cybernetics is about steering and getting to the goal, and "is the most powerful language to describe systems that have goals. That is the purpose of cybernetics" (Pangaro, 2012). Social, technical, biological, systems have goals, as do all living organisms.

\section{Deep Ecology}

Deep ecology is an ecological and environmental philosophy that does not separate humans or anything else from the natural environment. It brings together thinking, feeling, spirituality and action (Robert, 2011). It considers humans as an integral part of their environment and sees the world as an integrated whole rather than a dissociated collection of its parts (Robert, 2011). Capra (1996: 6) writes: "Deep ecological awareness recognizes the fundamental interdependence of all phenomena and the fact that, as individuals and societies, we are all embedded in (and ultimately depend on) the cyclical process of nature." Deep ecology sees the world as a subtle balance of complex inter-relationships of all living organisms. The core ideas of the deep ecology movement were formulated by Norwegian philosopher Arne Neass, who wasn't "the first to dream up the idea of a radical change in humanity's relationship to nature, but he coined the term 'deep ecology' and helped to give it a theoretical foundation" (Zimmerman, 1989). Arne Neass invented the term deep ecology in his article, "The Shallow and the Deep, Long-Range Ecology Movement: A Summary”, published in 1973.

Neass (1973) states that all living organisms have the right to live and flourish and that the right to live is a universal right, which cannot be quantified. The term "ecology" originates from the science of biology, which describes the interactions among organisms and their 
environment. For Naess (1973), facts and figures alone cannot answer ethical questions about how we should live. We need "ecological wisdom" to answer these questions. According to Naess (1973), we need to ask “deeper” questions, which may lead us to make deep changes in the way we look at ourselves, at the world and how we live. By doing so, we will create a deeper connection with life and nature. This also includes seeing ourselves as part of nature and not just as individuals alienated from nature and environment. Essentially, we are part of nature. As such, deep ecology focuses on:

deep experience, deep questioning and deep commitment. These constitute an interconnected system. Each gives rise to and supports the other, whilst the entire system is, what Naess would call, an ecosophy: an evolving but consistent philosophy of being, thinking and acting in the world, that embodies ecological wisdom and harmony (Harding, n.d.).

Deep ecology is grounded in eco-centric (Earth-centered) values, whereas the old paradigm is based on anthropocentric (human-centered) values (Capra, 1996: 11). Neass faults Western civilization "for the arrogance of its human-centered instrumentalization of nonhuman nature" (1973 as cited in Keller, 2009: 206). He sees mainstream environmentalism as merely an extension of Western anthropocentrism, whose "reasons for conserving wilderness and preserving biodiversity are invariably tied to human welfare, and it prizes nonhuman nature mainly for its use-value" (Keller, 2009: 206). Furthermore, Naess criticizes the arrogance of Western thought in its assumption that we humans exist to watch over nature like a highly respected middleman between the Creator and Creation. The deep ecology worldview, or paradigm, is much more radical and questions fundamental assumptions. This radical approach is also visible in the mission statement of The Foundation for Deep Ecology (n.d.):

We believe that stopping the global extinction crisis and achieving true ecological sustainability will require rethinking our values as a society. Present assumptions 
about economics, development, and the place of human beings in the natural order must be reevaluated. Nature can no longer be viewed merely as a commodity-a storehouse of "resources" for human use and profit. It must be seen as a partner and model in all human enterprise.

With this worldview, the inherent value of nonhuman life is acknowledged, and living organisms are bound together in a network of interdependencies: "When this deep ecological perception becomes part of our daily awareness, a radically new system of ethics emerges" (Capra, 1996: 11). With this new paradigm, where life plays the key role, we ask questions and experience nature. This results in our commitment to nature and a more environmentally sustainable way of living.

\subsection{Comparison of the two Paradigms}

The previous chapters outlined the mechanistic paradigm and the three branches (systems thinking, cybernetics and deep ecology) of a holistic, organic paradigm. It is quite visible that the organic worldview goes beyond personal materialistic growth and consumption. It goes beyond the anthropocentric approach and sees the human being as a part of the system, of nature, of the web of life. Whereas the modern paradigm tries to limit the observed as object of the perceiving human subject, the arising paradigm includes the whole instead of cutting things into pieces. It requires a completely new set of values and mindset where life itself is at the center. Relatedness and quality of relationships become important.

To give an overview of these two fundamentally different approaches, the following table offers a comparison: 


\begin{tabular}{|l|l|}
\hline Mechanistic Paradigm & Organic, Holistic Paradigm \\
\hline Mechanistic & Organic \\
\hline Machine & Living \\
\hline Parts, Pieces & Whole, Holistic, Systemic \\
\hline Structure & Order \\
\hline Matter & Pattern \\
\hline Quantity & Quality \\
\hline Result, Structure & Process \\
\hline Top-down & Bottom-up \\
\hline Domination, Competition & Collaboration \\
\hline Control & Adoption \\
\hline Predefined, Structured & Self-Organized, Spontaneous \\
\hline Static & Flexible \\
\hline Predictable & Uncertain \\
\hline Linear & Non-linear \\
\hline
\end{tabular}

[3.1] Comparison of the two Paradigms (author's illustration)

It is quite visible that the transition from the mechanistic to the organic paradigm requires a lot of trust and letting go of the rational mind. It is about non-linearity, unpredictability and trust in life. It is also obvious quality is more important than quantity. The process to reach a goal is important as well as the goal itself. It is more about the process and quality than the quantity. Western science insists upon an objective, reproducible measurement; the Eastern accepts our subjective experience as a valid measurement. Collaboration and selforganization become more important than control and predefined structures. Flexibility and creativity, including feelings and emotions aligned with actions are part of an organic worldview. We need actions based on both emotion and reason.

As we will see in the following chapters, many of the approaches and values of an organic paradigm are applied in the open source movement. 


\subsection{Conclusion}

In this and preceding chapters, we have seen that we are living a crisis of perception and values. Pre-modern, traditional forms of societies clash with the growing modern way of life, based on growth and expansion: "The social structures that we see decaying and crumblinglocally, regionally, and globally -are built on two different sources: pre-modern traditional and modern industrial structures of forms of thinking and operating. Both of them have been successful in the past. But in our current age each this integrates and crumbles" (Scharmer, 2009: 3). The fact that we are able to fix a fragment of a problem is obvious and possible, but only in a very limited way. Most problems are interconnected, where a linear approach is not enough. Koppensteiner (2010) used the words: “Modernity's dogmatism of reason has left large parts of the human potential untapped or denied." To go beyond linear mechanistic problem solving we cannot use our rational minds alone, but have to include our senses, feelings and emotions: "We must thoroughly understand the problem and begin to see the possibility of evolving a new lifestyle, with new methods of production and new patterns of consumption; a lifestyle designed for permanence” (Schumacher, 1979: 9).

Systems thinking shows us that everything is connected with everything, and we are only a part of a bigger system. The world, human beings and organizations are living beings and naturally in movement. A human being consists of about thirty billion components, which are constantly interchanging and in movement. All these elements in the system are interconnected. Spare parts do not exist (Robert, 2011).

Cybernetics is a language to describe regulatory systems, their structures and possibilities. Every living system is a regulatory system and tries to reach a goal. An example of a goal of a living system is keeping its temperature in a healthy range. Cybernetics looks at the relationships in between these system components and does not look at its materialistic 
composition. It is about the interrelation of its components, where relationships become important.

Deep ecology questions our whole way of life from an ecological perspective: "from an ecological perspective, from the perspective of our relationships to another, to future generations, and to the web of life of which we are part" (Capra, 1996: 8). Deep ecology includes our experience and feelings toward nature and argues for a whole new set of values for a more environmentally sustainable life.

All of these approaches are beyond the rational Newtonian paradigm in which we currently live, and which has severe implications on the environmental health of this planet and its humanity. I close this chapter with a powerful quote from Ludwig von Bertalanffy (1968: 45): "There is this hope, I cannot promise you whether or when it will be realized-that the mechanistic paradigm, with all its implications in science as well as in society and our own private life, will be replaced by an organismic or systems paradigm that will offer new pathways for our presently schizophrenic and self-destructive civilization." 


\title{
4 Open Source and Living Systems
}

\author{
Revolution doesn't happen when society adopts new technology- \\ it happens when society adopts new behaviors.
} -- Clay Shirky $(2009,160)$

For a few years now, we have had the possibility to connect effectively on a low-cost level and without geographical barriers through new technologies like the Internet and mobile phones. We are able to collaborate more easily, and we are developing software around the globe - 24 hours a day, in shifts, in different time zones. Particularly during the last few years, new software technologies have reshaped how we communicate, connect and collaborate. Examples like the free encyclopedia of Wikipedia show how we are able to gather, use and permanently rewrite knowledge in a global, non-hierarchical and open way. This tendency has occurred in software development for several years now, and is also starting to expand into hardware development. Currently, the number of open source (hardware) projects is increasing every day, where people with the same interest in finding a technical solution for a problem gather, develop and innovate in a collaborative way.

This chapter explores the approach of open source, covering the areas of open source ideas and projects in software, as well as in hardware. Some sections also deal with the technical possibilities of new media, which are fundamental to enabling the open source approach. Additionally, the theoretical background of networks and living systems is applied to selforganization and non-hierarchy. Throughout this chapter, I maintain the analogy between the open source approach and an organic, holistic paradigm based on life, as you will see. Building on a definition and introduction of open source, the following pages elaborate on the approach from a living, organic paradigm perspective, while investigating the subject from different angles. 


\subsection{Introduction to Open Source}

Open Source is a growing movement of the $21^{\text {st }}$ Century. It originally grew out of the Free Software movement, "which laid out the groundwork and developed methods for collaboration around a common pool of information and resources” (Open it Agency, n.d.). Like most definitions, it is difficult to define the term "open source", and is probably impossible to find a general definition. The term open source itself refers to the humanreadable code of software. Therefore, open source implies the accessibility to the "source" of a product. As software is a digital good, the documentation, source code and design can be made open entirely.

But the term 'open source' describes also a social movement, a development methodology, and/or perhaps some sort of underlying values held in common. Open source means new methods for communication and collaboration. Ronald Wyllys (2001: 1) used the following words to describe the open source movement:

Briefly, it is a world-wide movement composed, both formally and informally, of many people who feel that the best way to produce software that will be sophisticated, robust, and (relatively) bug-free is to enlist the cooperation of interested, skilled, altruistic programmers who are willing to work for free, inspired by the twin goals of producing high-quality programs and of working cooperatively with other similarly minded people.

The Open Source Initiative (OSI) ${ }^{9}$ defines open source as much more than just the access it offers to the source code of particular software. So, what is a source code? Basically a source code is text, written in programming language. This code is translated into a machinereadable version by a "compiler". After this compilation, a machine is able to understand the

\footnotetext{
${ }^{9}$ The OSI is a non-profit corporation with global scope formed to educate about and advocate for the benefits of open source and to build bridges among different constituencies in the open source community (Open Source Initiative, n.d.). Source: OSI Mission: http://www.opensource.org/, retrieved January 16, 2012)
} 
code and execute its orders. Proprietary software is generally delivered only in one machinereadable way, which means that the buyer or "owner" of the software is not able to observe the actual processing of the software, as he is not able to read the language. This means that the buyer of the software only acquires the license that allows him or her to use the software under certain conditions. Richard Stallman, the founder of GNU not UNIX ${ }^{10}$, and the General Public License states that: "Proprietary software keeps users helpless and dependent on the producer of the software” (Stallmann, 2011a). Furthermore:

Proprietary software means, fundamentally, that you don't control what it does; you can't study the source code, or change it. It's not surprising that clever businessmen find ways to use their control to put you at a disadvantage. Microsoft has done this several times: one version of Windows was designed to report to Microsoft all the software on your hard disk (Stallman, 2007).

The core idea of open source is rooted in conflicts with and reactions towards these limitations. People wanting to change minor functions of software were not able to do so because of its limited access and license. This formed the initial motivation for demands to be able to access the source code; and in combination with "bad news" about "company backdoors" in software, the open source movement became popular. The open source movement received another push after the publication of whistleblower Edward Snowden.

The need for a license, a categorization or definition became important, and the Open Source Initiative determined that open source software had to comply with the following principles (Open Source Initiative, n.d.):

- Free Redistribution

- Derived Works

\footnotetext{
${ }^{10} \mathrm{GNU}$ is a Unix-like operating system, which is free software. A Unix-like operating system is a software collection of applications, libraries and developer tools, plus a program to allocate resources and talk to the hardware, known as a kernel. (Source: Free Software Foundation, http://www.gnu.org/, retrieved April 4, 2012Free Software Foundation, n.d.)
} 


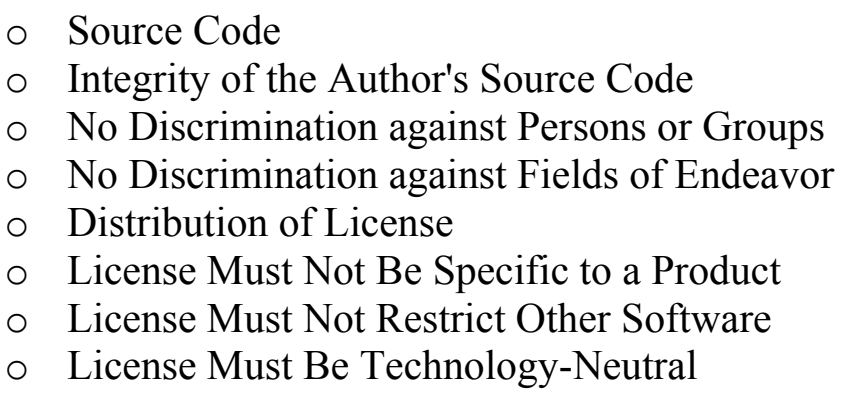

Instead of open source software, Richard Stallman also speaks about "free software", a term he created (Stallman, 2011a). This term is slightly different from that of open source, but is very closely related and essentially just boils down to a personal preference of wording. "Free software" is a matter of liberty, not price. To understand the concept, one should think of the idea of "free" as in "free speech", and not as in "free beer" (GNU Operating System, 2010). Stallman (2011a) defines four types of freedom towards software that allow the user to run, copy, distribute, study, change and improve on it. The four freedoms are:

- The freedom to run the program, for any purpose (freedom 0 ).

- The freedom to study how the program works, and change it so it does your computing as you wish (freedom 1).

- The freedom to redistribute copies so you can help your neighbor (freedom 2).

- The freedom to distribute copies of your modified versions to others (freedom 3) (GNU Operating System, 2010).

These freedoms are only accomplishable if the source code is open and available to any user. Therefore, the availability of the source code (open source) is a precondition to free software.

Furthermore, open source is the basis for a movement beyond software development. This movement is spreading towards many different areas of our lives as a process happening on a global scale, in which new technologies of communication, and particularly the Internet and social networks, play a crucial role. The following numbers only depict the crucial change we will face with these new technologies. Facebook, the most popular social media platform worldwide, reached 1.7 billion active users in the second quarter of 2016 . More than 600 
million people use social networks at least daily. Google+ needed only 16 days to reach 10 million users at the time of research. If the social network Facebook were a country, it would be the third largest in the world. ${ }^{11}$

People use social networks to stay connected with friends and family, mostly when they are living far away. These social networks, where mainly young people share ideas and interact across borders, have only recently emerged. This is happening in different languages and on a global scale; a current technical development connecting people in a way that was never possible before. At the same time, this enables people not only to communicate, but also to co-create and collaborate in a different way, beyond geographical and temporal constraints. For instance, a small team of developers could consist of people from different continents and countries all over the world, which again, to some extent, fosters creativity and flexibility. This specific topic will be covered more extensively in the chapter on the characteristics of open source projects.

The open source movement started with software. Software development is a specific field of production whereby no investment is necessary for participation. In general terms, everyone with a computer is able to participate in the development process and contribute an element to a software product. This might include some lines of code, a translation of documentation, or whatever is necessary.

\subsubsection{Open Source Software}

The range of open source software covers almost every application imaginable, including the operating system Linux, which is probably one of the most stable operating systems in the

\footnotetext{
${ }^{11}$ Statistics retrieved from (http://www.statista.com/topics/1164/social-networks/), August 08, 2016. / Pingdom Website Monitoring (http://royal.pingdom.com/2011/02/04/facebook-youtube-our-collective-time-sinks-stats/), / Jeffbullas.com (http://www.jeffbullas.com/2011/09/02/20-stunning-social-media-statistics/), / Alexa - Web Information (http://www.alexa.com/siteinfo/facebook.com\#), all accessed on April 14, 2012
} 
world. Surprisingly, the market share (of desktop operating systems) of Linux is only around 2\% (Netmarketshare, 2016a). However, this does not mean that it is not influential within that market segment; rather, the opposite is true. Linux is mostly used by governments, organizations and institutions who want to have full access and control of the software they use, similar to the four freedoms mentioned previously. The following examples show how and where Linux is used:

- U.S. Department of Defense (According to Linux.com, the United States Department of Defense is the "single biggest install base for Red Hat Linux" in the world. [...] as Brigadier General Nick Justice, the Deputy Program Officer for the Army's Program Executive Office proclaims "open source software is part of the integrated network fabric which connects and enables our command and control system to work effectively, as people's lives depend on it." Justice went on to state that "when we rolled into Baghdad, we did it using open source", and that he was indeed Red Hat's "biggest customer.")

- Google (Believe it or not, the gigantic, ever-growing cluster of servers that power Google's search and other apps runs Linux. Of course, in typical fashion, Google was not content to simply run an out-of-the-box version on its own hardware. Instead, the search giant had its engineers cook up a customized version of Ubuntu referred to within the company as "Goobuntu." Linux is also frequently used internally on desktop machines, beyond its use on Google servers.) (Focus, 2011).

Amazon, The U.S Navy Submarine Fleet, The City of Munich (Germany), Novell, IBM, Panasonic, Cisco, Peugeot, Wikipedia and many Universities, schools and governmental institutions all over the world are using and, apparently, trusting Linux more than a proprietary operating system (Focus, 2011). Most technical devices use a Linux-based operating system. The open source operating system "Android" accounts for 66 percent of the worldwide smart phone market share (Netmarketshare, 2016b). This is also the case with all other kinds of software, including applications for sectors such as healthcare, media, security, computer-added design, education, groupware, games, data management, etc. (Open Source List, n.d.). 
One of the most popular examples demonstrating the success of open source software is the web browser Firefox, promoted by the Mozilla Foundation. ${ }^{12}$ Its market share is set around 25 percent and many web developers favor this browser because of the given freedom to add whatever little program or function you wish (Netmarketshare, 2011).

Another example is Open Office, whose market share in Germany, Austria and Switzerland is 21 percent (WebmasterPro, n.d.), representing just one of the numerous open source office applications.

Open source content management systems (CMS) are another striking example, and include Wordpress, Joomla and Drupal, whose combined market share is 84 percent, and there are many more open source CMS in existence) (Builtwith, n.d.).

The Apache Server contributes perhaps one of the most impressive performances. The Apache Server is an open source webserver that runs on approximately 50 per cent of all webservers on the internet, as the graph on the following page demonstrates.. Law professor Yochai Benkler explains the development of the Apache Server in his TED ${ }^{13}$ talk in the following words:

In 1995, two groups of people said wow, this is really important, the web! We need a much better web server! One was a motley collection of volunteers who just decided you know, we really need this, we should write one, and what are we going to do with what - well, we're gonna share it! And other people will be able to develop it. The other was Microsoft. Now if I told you that 10 years later, the motley crew of people who didn't control anything that they produced acquired $20 \%$ of the market it would be amazing! Right? Think of it in minivans. A group of automobile engineers on their weekends are competing with Toyota. Right? (Benkler, 2005).

\footnotetext{
12 The Mozilla Foundation is a non-profit organization that promotes openness in the internet and supports and leads software projects, such as the web browser Firefox.

${ }^{13}$ Technology Entertainment Design (TED) is a non-profit organization devoted to ideas worth spreading. It started as a conference, and due to the exchange of technology online, talks are available on their website, www.ted.com.
} 
What no one knew at that time and what is still amazing today is that ten years later, in 2015, the market share of the Apache web server is still at 50 per cent. It is important

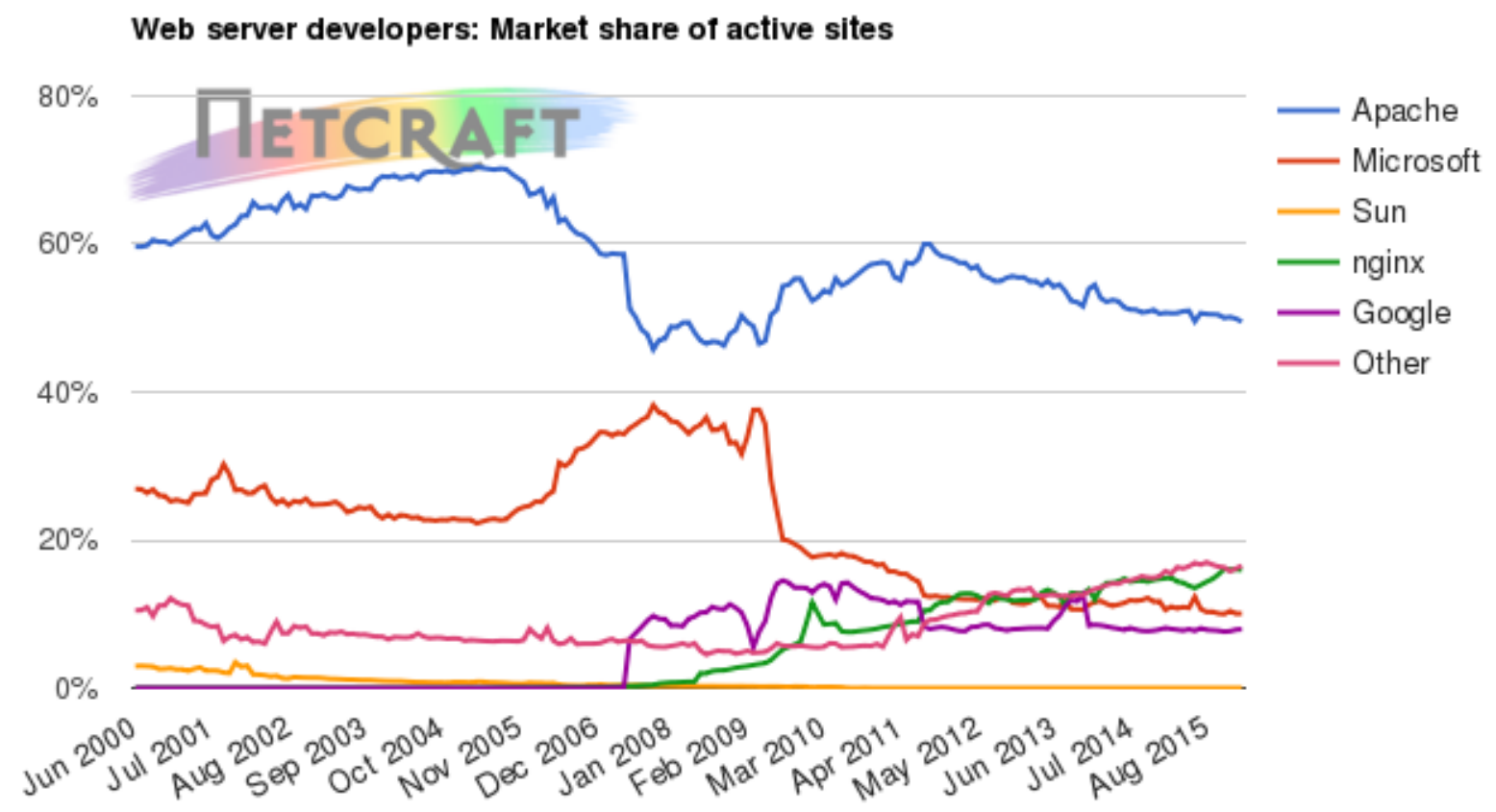

[4.1] Web server developers: Market share of active sites (Netcraft, 2016)

to mention the fact that a remarkable loss of market share is due to the fact that a new open source player, namely $\mathrm{NGINX}^{14}$, is gaining market share. With only a few years on the market, it is already close to being the second most important webserver, worldwide.

The model of open source software development has proven to be extremely successful: "Today most of the technical foundations of the internet are Free / Libre / Open Source Software and it is found throughout the software portfolios of most large enterprises" (Open it Agency, n.d.). Now that we have explored the origins and several key examples of open source software "success" projects, the next chapter will cover open source hardware projects, one field where the open source model is expanding its reach.

\footnotetext{
${ }^{14}$ NGINX is a free, open-source, high-performance HTTP server and reverse proxy, as well as an IMAP/POP3 proxy server. (Retrieved August 08, 2016 from www.nginx.com, n.d.) 


\subsubsection{Open Source Hardware}

Open source hardware gives people the freedom to control their technology while sharing knowledge and encouraging commerce through the open exchange of designs. - Open Source Hardware Association (n.d.)

As we have seen, there are many successful open source software projects in existence, and this approach seems to work with virtual products, namely with software. But is it also feasible in the hardware world? What is the difference between hardware and software?

Open Source Hardware (OSH) is hardware whose design is made publicly available so that anyone can study, modify, distribute, make, and sell the design or hardware based on that design. The highly decentralized and local approach gives people the freedom to control their technology while sharing knowledge. This enables users to contribute their experience during and after the design process. Therefore, OSH users become "prosumers" - both producers and consumers at the same time, which has a fundamental influence on the intention (product design and usage) and provokes the creation of a decentralized yet connected network of "prosumers".

OSH has become a reality because of the Internet, new media and recently developed communication technologies. Today, it is possible in less-developed countries to access Internet, download a "How-to build whatever" manual and "feedback" one's local experience into the community at the same time. Furthermore, this means that the intention of the product development is not commercially or profit-driven. It is more about a common irk or a fundamental passion to create a product as sustainable as possible and to avoid a low-cost solution intended to make the highest profit possible. The product is born out of a need and not a potential market. 
OSH can be described as a "second generation" of open source software, as the core idea is basically the same. The difference lies within the product itself, which is hardware. In OSH the actual product is physical, only the documentation is digital and can be shared. The hardware layout and design (i.e. manufacturing drawings, mechanical drawings, bill of materials, schematics, documentation, manuals, etc.) are entirely released, published for free and with the intent to share. All these documents for hardware are comparable with the source code of open source software. By sharing and collaborating on this information, products can be improved fast and everyone is able to contribute his/her part and unique experience, just as in the open source software world. Open source hardware started only a few years ago, but there are already many projects on the way, and its presence and impact increase daily. This includes cameras, computer components (cpus, graphic cards, etc.), audio and video electronics, all kinds of machines, tools, automotive and automation (P2P Foundation, n.d.).

Within the last few years, the open source hardware movement created a legal and organizational framework equivalent to, and already established, for open source software. The combination of the four freedoms and the open source hardware definition are two of the main frameworks for this movement.

The open source hardware association is a not-for-profit organization, aiming "to be the voice of the open hardware community, ensuring that technological knowledge is accessible to everyone, and encouraging the collaborative development of technology that serves education, environmental sustainability, and human welfare” (OSHWA, n.d.). The Open Source Hardware Association (OSHWA) defines open source hardware as "hardware whose design is made publicly available so that anyone can study, modify, distribute, make and sell 
the design or hardware based on the design" (OSHWA, n.d.). The OSHWA definition "serves as a set of agreed-upon standards for the main characteristics that open source hardware must have to be defined as open source hardware" (Gibb, 2015: 13). Furthermore, the OSHWA Definition is a "social contract that the open hardware community has agreed to uphold. It allows the community to have agreement on decisions such as whether open source hardware can be created with a noncommercial clause attached (it cannot) and whether the source files must be free of charge and accessible via a website (they must)" (Gibb, 2015: 13).

In September 2011, during the second Open Source Hardware Summit ${ }^{15}$ in New York, people from all over the world met to exchange ideas and define open source hardware. On Freedom Defined's website, freedomdefined.org ${ }^{16}$, the so-called "Open Source Hardware Definition" is available in version 1.0. It covers the following twelve points and strongly emphasizes the open source software definition outlined in the previous chapter:

1) 1. Documentation: The hardware must be released with documentation including design files, and must allow modification and distribution of the design files. Where documentation is not furnished with the physical product, there must be a wellpublicized means of obtaining this documentation for no more than a reasonable reproduction cost, preferably downloading via the Internet without charge. The documentation must include design files in the preferred format for making changes, for example the native file format of a CAD program. Deliberately obfuscated design files are not allowed. Intermediate forms analogous to compiled computer code such as printer-ready copper artwork from a CAD program - are not allowed as substitutes. The license may require that the design files are provided in fullydocumented, open format(s).

2) 2. Scope: The documentation for the hardware must clearly specify what portion of the design, if not all, is being released under the license.

3) 3. Necessary Software: If the licensed design requires software, embedded or otherwise, to operate properly and fulfill its essential functions, then the license may

\footnotetext{
${ }^{15}$ The Open Source Hardware Summit is a New York-based yearly conference on open hardware, covering all kinds of topics regarding open hardware, such as: $\mathrm{OH}$ in education, $\mathrm{OH}$ business models, digital fabrication, industrial design, competition and collaboration, etc.

${ }^{16}$ At freedomdefined.org, the Definition of Free Cultural Works is defined and published.
} 
require that one of the following conditions are met: a) The interfaces are sufficiently documented such that it could reasonably be considered straightforward to write open source software that allows the device to operate properly and fulfill its essential functions. For example, this may include the use of detailed signal timing diagrams or pseudocode to clearly illustrate the interface in operation.

b) The necessary software is released under an OSI-approved open source license.

- 4. Derived Works: The license shall allow modifications and derived works, and shall allow them to be distributed under the same terms as the license of the original work. The license shall allow for the manufacture, sale, distribution, and use of products created from the design files, the design files themselves, and derivatives thereof.

4) 5. Free redistribution: The license shall not restrict any party from selling or giving away the project documentation. The license shall not require a royalty or other fee for such sale. The license shall not require any royalty or fee related to the sale of derived works.

5) 6. Attribution: The license may require derived documents, and copyright notices associated with devices, to provide attribution to the licensors when distributing design files, manufactured products, and/or derivatives thereof. The license may require that this information be accessible to the end-user using the device normally, but shall not specify a specific format of display. The license may require derived works to carry a different name or version number from the original design.

6) 7. No Discrimination Against Persons or Groups: The license must not discriminate against any person or group of persons.

7) 8. No Discrimination Against Fields of Endeavor: The license must not restrict anyone from making use of the work (including manufactured hardware) in a specific field of endeavor. For example, it must not restrict the hardware from being used in a business, or from being used in nuclear research.

8) 9. Distribution of License: The rights granted by the license must apply to all to whom the work is redistributed without the need for execution of an additional license by those parties.

9) 10. License Must Not Be Specific to a Product: The rights granted by the license must not depend on the licensed work being part of a particular product. If a portion is extracted from a work and used or distributed within the terms of the license, all parties to whom that work is redistributed should have the same rights as those that are granted for the original work.

10) 11. License Must Not Restrict Other Hardware or Software: The license must not place restrictions on other items that are aggregated with the licensed work but not derivative of it. For example, the license must not insist that all other hardware sold with the licensed item be open source, nor that only open source software be used external to the device. 
11) 12. License Must Be Technology-Neutral: No provision of the license may be predicated on any individual technology, specific part or component, material, or style of interface or use thereof (Freedom Defined, n.d.).

Correspondingly, the "four freedoms" already established by open source software practice are adapted to open source hardware. The four freedoms in OSH are:

○ Freedom 0: The freedom to use the device for any purpose

- Freedom 1: The freedom to study how the device works and change it to make it to do what you wish. Access to the complete design is a precondition to this.

○ Freedom 2: The freedom to redistribute the device and/or design (remanufacture).

- Freedom 3: The freedom to improve the device and/or design and release your improvements (and modified versions in general) to the public so that the whole community benefits. Access to the complete design is a precondition to this (OHANDA, n.d.).

The Open Hardware and Design Alliance (OHANDA) ${ }^{17}$ provides a label for open hardware projects: "The four freedoms from Free Software Definition lay the foundation for sharing hardware through OHANDA" (OHANDA, n.d.). This label associates the four freedoms with any kind of physical device in the sense of a non-registered trademark. A product registered with OHANDA receives a unique key, which ties that product to a unique $\mathrm{URL}^{18}$. The idea is to engrave the URL on each physical product so that other people are able to access documents and information about the product and its license. The OHANDA label, depicted below, will conform with the Conformité Européenne (CE) norm, which ensures that the

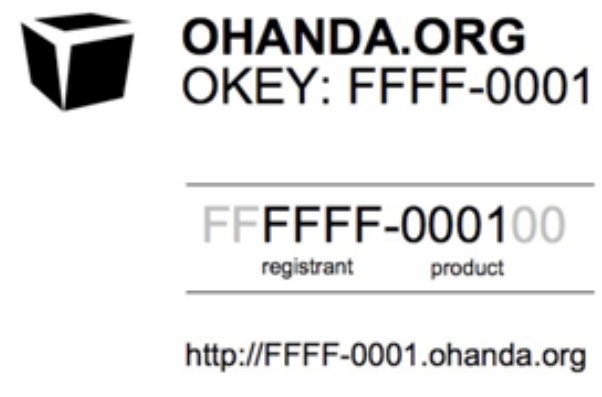

[4.2] OHANDA Product Identification Label (OHANDA, n.d.)

${ }^{17}$ OHANDA is an initiative to foster sustainable sharing of open hardware and design.

${ }^{18}$ URL - Uniform Resource Locator, is the address where a webpage is available on the World Wide Web. 
product conforms to the requirements of the applicable European Community directives. The idea behind this approach is to make the openness of physical hardware visible and accessible to everyone, while at the same time establishing quality and security standards.

The four freedoms and the open source hardware definition build the framework for OSH development. Following this framework for product licensing, many companies and individuals have built successful businesses: "The open source hardware community is continually trying to standardize documentation and efficient sharing of work related to a piece of hardware so that others can use and modify the work, and it encourages the development of a vibrant community of people who use and improve on other open source hardware projects" (Gibb, 2015: 150).

Open source hardware embodies the core practice of open source: "To share information openly - and in doing so, empower people and give them the freedom to understand, adapt and improve the objects and tools they interact with. This process enables unique opportunities and new forms of direct and indirect collaboration that can be used by companies to grow their business and enhance their standing with the community" (Open it Agency, n.d.).

With this understanding of the legal and organizational background, the following chapter elaborates on the diversity of open source hardware projects.

\subsubsection{Open Source Hardware Projects}

Especially during the last few years, most likely stimulated by the development of communication technology, open source hardware projects increased in number and developed more intensely. The application range of open source hardware is as broad as in 
open source software. In almost all sectors open source hardware projects and initiatives can be found, "including industrial machines, agriculture, mechanical engineering, manufacturing, transportation, education, sustainable energy and robotics” (Gibb, 2015: 134).

By now there are also online stores and shops like Sparkfun or Willow Garage available, which specialize in open hardware products. Adafruit Industries, founded in 2005, is one of the biggest stores for "Unique \& Fun DIY Electronics and Kits" (Adafruit, n.d.). At the annual hacker conference, Foo Camp ${ }^{19}$ 2010, Phillip Torrone and Limor Fried from Adafruit Industries gave a presentation on thirteen companies that generate million-dollar revenues by working with open source hardware. Torrone and Fried estimated that the industry will reach a billion dollars by 2015 (Singularity Hub, n.d.). This estimation is not limited to hardware itself, but also involves other business branches, like magazines and online communities, such as MAKE magazine. MAKE is a magazine for the open source hardware community that “unites, inspires, informs, and entertains a growing community of resourceful people who undertake amazing projects in their backyards, basements, and garages” (Make, n.d.).

For further understanding, the following pages offer some categorical examples of available open source hardware, licensed according to OSHWA principles.

\section{Automotive Industry:}

The Italian company OSVehicle developed a small open source vehicle named Tabby. A complete set of assembly drawings for the car are available to download, and "according to the company, it can be assembled in less than an hour in a customer's garage for less than

${ }^{19}$ Foo Camp is an annual hacker event hosted and initiated by the publisher O'Reilly Media. 
6000 Euros" (Gibb, 2015: 138). In their centralized community forum, customers can share improvements and make modifications to the design, which facilitates crowd-sourced research and development. This forum also serves as an online hub for communication and global collaboration (Gibb, 2015: 138).

Velocar is a platform for an open source bicycle that has a protective shell to shield the rider from the elements (rain, cold and wind, for example). It takes the form of a Velomobile, which is a tricycle covered by an aeroshell (Gibb, 2015: 138).

\section{Agriculture:}

Windowfarms is a hydroponic urban indoor vertical gardening system "made from repurposed water bottles and accessible plumbing supplies that deliver liquid nutrients to plant roots" (Gibb, 2015: 134). The windowfarm system allows the growing of plants in the window of any house or apartment, and "the plants thrive on natural light from the sun, coming into the window"(Gibb, 2015: 134). Similarly:

Along with a store for purchasing Windowfarms and an online wiki detailing how to build, set up, and maintain Windowfarms, a centralized social media community sharing website allows users to share their improvements to the design. To date, the Windowfarms community has grown to more than 42,000 users worldwide who have contributed to the online forum about modifications to the technology. This crowdsources development has facilitated rapid technology advancements to the point where it is today inexpensive and effective (Gibb, 2015: 135).

AKER is developing methodologies, tools and kits to integrate natural agricultural and ecological systems with urban environment "in order to restore balance to food production and consumption cycles" (Shuttleworth, n.d.). AKER includes garden kits to grow vegetables on your balcony and sensor enhanced beehives. The open source beehive sensor tries to 
monitor "honeybee-health indicating factors inside a beehive, and share the collected data on the Internet" (Open Source Beehives, n.d.). The goal behind this project is to make it easy for any beekeeper to gather information on the health of honeybee colonies and share it in a decentralized and transparent manner.

\section{Mechanical Engineering and Manufacturing:}

Lasersaur is an open source laser cutter designed and developed by an international research and development studio named Nortd Labs ${ }^{20}$ located in New York City; as stated on their website: "We designed it to fill the need of makers, artists and scientists who wanted a safe and highly capable machine" (Lasersaur, n.d.). The open source laser cutter "with outstanding price-performance ratio" can be produced using modular parts that can be sourced directly or bought locally by referencing the bill of materials (Gibbs, 2015: 138).

The distribution of the Lasersaur is based on its comprehensive documentation and bill of materials (BOM). With this approach it is possible to build a Lasersaur with locally sourced parts. The online documentation covers the resources needed for building and maintaining the Lasersaur, including hardware/software specifications, the source code, the building process, and instruction on how to run and operate the laser cutter. Additionally, there is a mailing list and a map of users. Gibb (2015: 138) describes the positive aspects of using open source hardware as follows:

The benefit of using open source hardware during the development process is that the Nortd Lab team was able to release the design sooner to the public. The hope was that open collaboration would help its design improve over time as people used it, contributed to the development, and documented improvements. Instead of putting years of effort and money into R\&D, Nortd Labs shared the design with the public at an early stage in the development. In turn, hackerspaces and universities participated

\footnotetext{
${ }^{20}$ Nortd Labs is a research and development collaboration-based studio of creative thought with a focus on art, architecture and system design.
} 
in the collective research and development. This crowd-sourced R\&D enabled tools that were typically created in a hierarchical development cycle to be developed by an international online network composed of academics, hobbyists, artists and scientists.

MiniCNC, as the name implies, is a small computer numeric controlled (CNC) milling machine (Repairable Machines, n.d.) developed by TimeLab, ${ }^{21}$. "The iniCNC can be assembled by a novice builder from the kit parts in 14 hours; that is, from a box of parts to a working machine in one weekend", Lieven Standeart ${ }^{22}$ told me at the 2011 Open Knowledge Conference in Berlin. The following photo depicts a miniCNC machine, presented at the Open Knowledge Conference 2011.

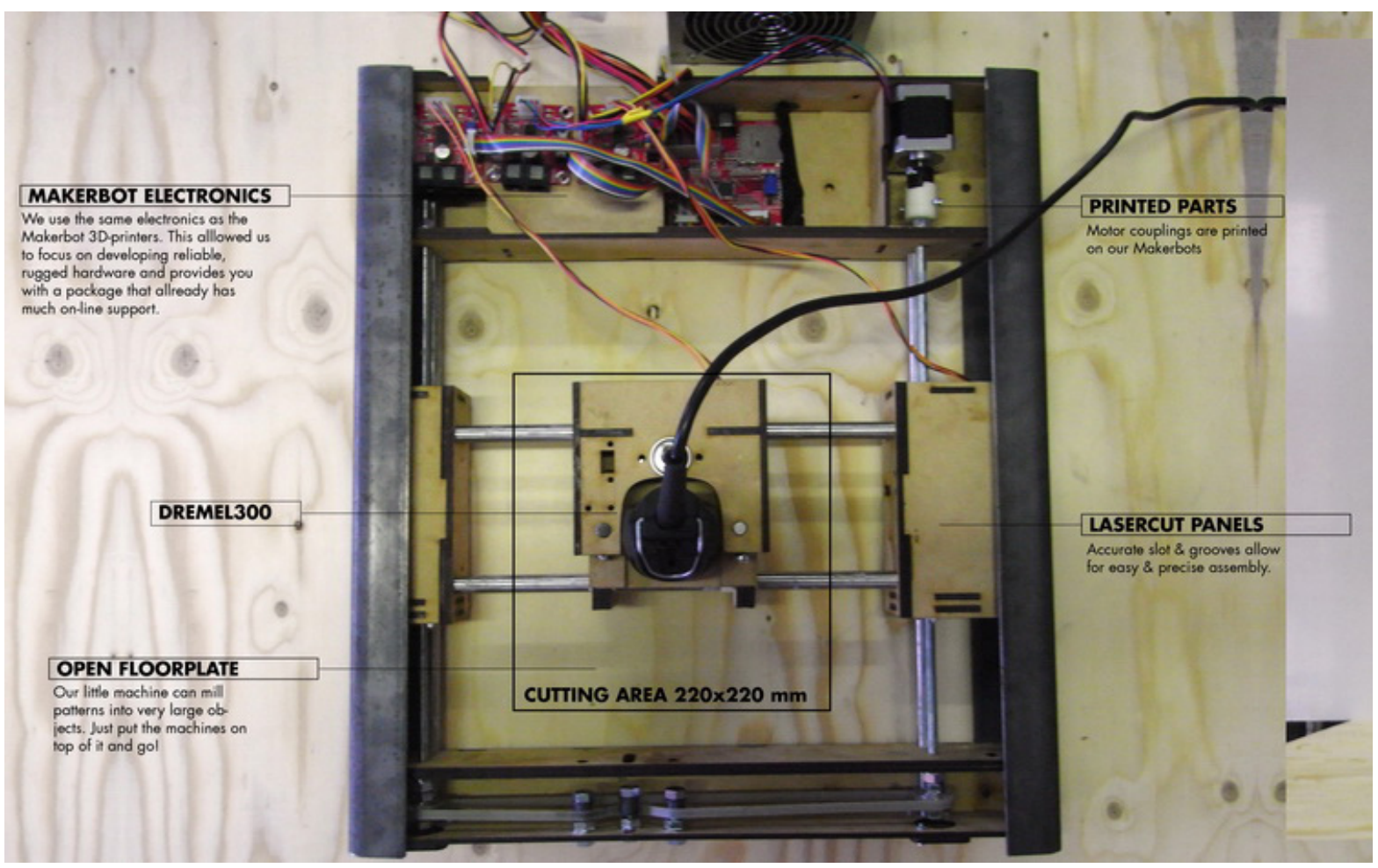

[4.3] MiniCNC from TimeLab (TimeLab, n.d.)

Another open source CNC milling machine named Shaproko was developed in California, USA: "The Shapeoko 3 comes with everything you need to make accurate parts from wood,

\footnotetext{
${ }^{21}$ TimeLab is a workplace for art, technology and society in Ghent, Belgium. It is a so-called hackerspace, including an artist in residence program, summer camps and much more.

${ }^{22}$ Lieven Standeart is one of the core developers of the miniCNC
} 
plastic, and aluminum" (Carbide 3D, n.d.). The parts required to build the modular, upgradeable, easy-to-assemble, low-cost CNC router, are available online through a bill of materials. The whole package can also be purchased online as a preassembled consumerfriendly kit (Gibb, 2015: 138): "You can built in it 1 hour" (Carbide 3D, n.d.). The other distribution channel is through the BOM and made with locally sourced products similar to the Lasersaur project, as already explained. The control unit is Arduino based, so the microcontroller is well known and accessible. The device's shape and bed size can be expanded or remolded based on the original design (Gibb, 2015: 138).

\section{D Printing:}

Numerous open source 3D printers have been developed within the last few years. The following picture shows the model Thing-O-Matic from MakerBot Industries, based in New York, which was a very popular open source 3D printer for the hobbyist and consumer market with a huge online community involved before it was bought by Stratasys.

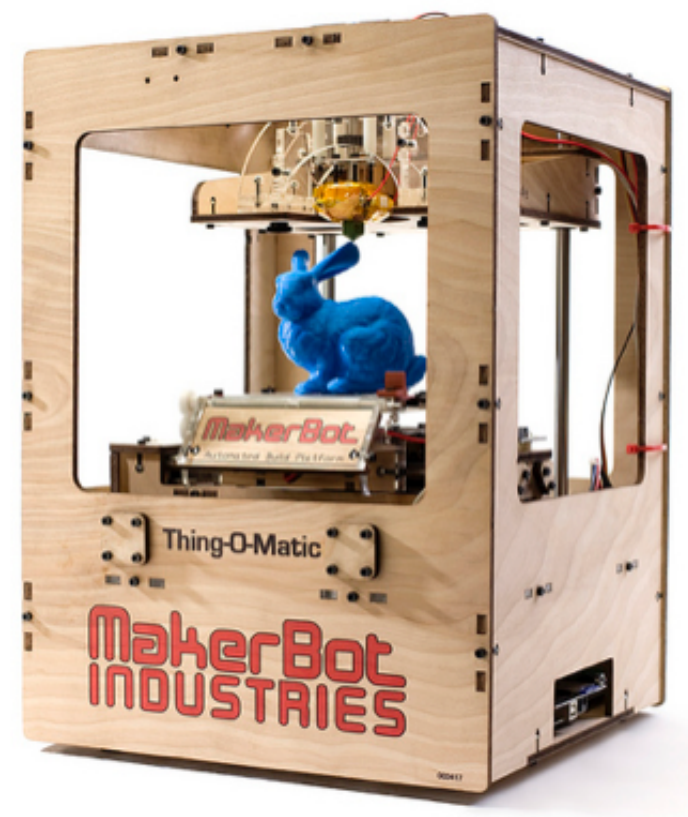

[4.4] Thing-O-Matic from MakerBot Industries (Brown, 2012) 
The $3 \mathrm{D}$ printing market is vibrant, and a wealth of derivates ${ }^{23}$ has emerged, with newer models being built, derived, modified and rebuilt. The influence of open source on 3D printing was pointed out by Alicia Gibb through the following words: "It is important to note that the consumer $3 D$ printing market would not exist as it does today without the RepRap $3 D$ printer being open source hardware, as the vast majority of consumer printers on the market are derivatives of the RepRap" (Gibb, 2015: 140).

\section{Air and Space Exploration:}

Chris Anderson is the founder of the largest web-based community for modular flying robots (DIY Drones) and the co-founder of the largest DIY drones distributor, 3D Robotics: "The DIY drones website has spawned a growing group of enthusiasts, composed of hundreds of developers, who have developed one of the largest Arduino programs collaboratively" (Gibb, 2015: 142). The 3D Robotics drone platform is now used worldwide by hobbyists and companies all over the world for things like unmanned aerial photography, mapping, data collection and transportation (Gibb, 2015: 142).

\section{Environmental Preservation, Disaster Relief and Mapping:}

OpenRelief is a platform that aims to enhance disaster relief efforts by developing communication tools to facilitate getting aid to the right place at the right time. Open Relief designed an inexpensive, semi-disposable open source hardware drone with the aim to map disaster zones. As stated on their website "the device can be launched from a small road" and Open Refief "ensures everyone, anywhere, can access our technology. We are working to complement other projects around the world to support the emergence of open, humanitarian drone technology” (OpenRelief, n.d.).

\footnotetext{
${ }^{23}$ A derivate is open source hardware that has been altered or modified but is based on an original design by another person or company.
} 


\section{Micro Controller:}

One of the best-known open source hardware projects started in Italy and is called Arduino. A search of the term "Arduino" via the search engine Google results in more than 55 million hits. According to the Arduino website:

Arduino is an open-source electronics prototyping platform based on flexible, easy-touse hardware and software. It's intended for artists, designers, hobbyists, and anyone interested in creating interactive objects or environments. Arduino can sense the environment by receiving input from a variety of sensors and can affect its surroundings by controlling lights, motors, and other actuators. The boards can be built by hand or purchased preassembled; the software can be downloaded for free. The hardware reference designs (CAD files) are available under an open-source license; you are free to adapt them to your needs (Arduino, n.d,).

Arduino is a striking example of how open source business models work. Today, various companies, most of them located in China, build and sell the hardware designed by the Arduino community.

It seems that open source hardware is an increasingly popular category of business, and many people are involved. But there is one aspect that all of the projects mentioned above have in common: none of them cover basic human needs, such as clean water, food, energy, etc. A laser cutter or 3D printer will primarily be built and used by people from the "Global North" 24 . These existing projects evidently follow the idea of open source and aim to build something that is affordable, customizable and more sustainable. However, they apparently tend not to contribute to reaching the United Nations Millennium Development Goals or helping people who lack technology satisfy their basic needs. There are only very few projects covering technologies for basic needs. One of them is open source ecology (OSE).

\footnotetext{
${ }^{24}$ For our purposes, the "Global North" refers to countries that rank higher than 7 on the UNDP Human Development Index. Most of these countries are geographically located in the north.
} 
The founder of the open source ecology group, Marcin Jakubowski, shares his motivation and vision in a TED talk from 2011:

We know that open source software has succeeded with tools for managing knowledge and creativity. And the same is starting to happen with hardware too. We are focusing on hardware, because it is hardware, which changes people's lives in such tangible and material ways. If we can lower the barriers to farming, building, manufacturing then we can unleash just massive amounts of human potential (Jakubowski, 2011).

Open source ecology develops open source industrial machines that can be made for a fraction of their commercial costs, with the ultimate goal of creating an efficient open source process to increase innovation through open collaboration (Gibb, 2015: 134). OSE is designing, developing and building the so-called Global Village Construction Set (GVCS), as stated on OpenSourceEcology.org: "The GVCS is a modular, DIY, low-cost, highperformance platform that allows for the easy fabrication of the 50 different Industrial Machines that it takes to build a small, sustainable civilization with modern comforts" (Open Source Ecology, n.d.). The following two pictures show the tractor and the whole portfolio of the Global Village Construction Set:

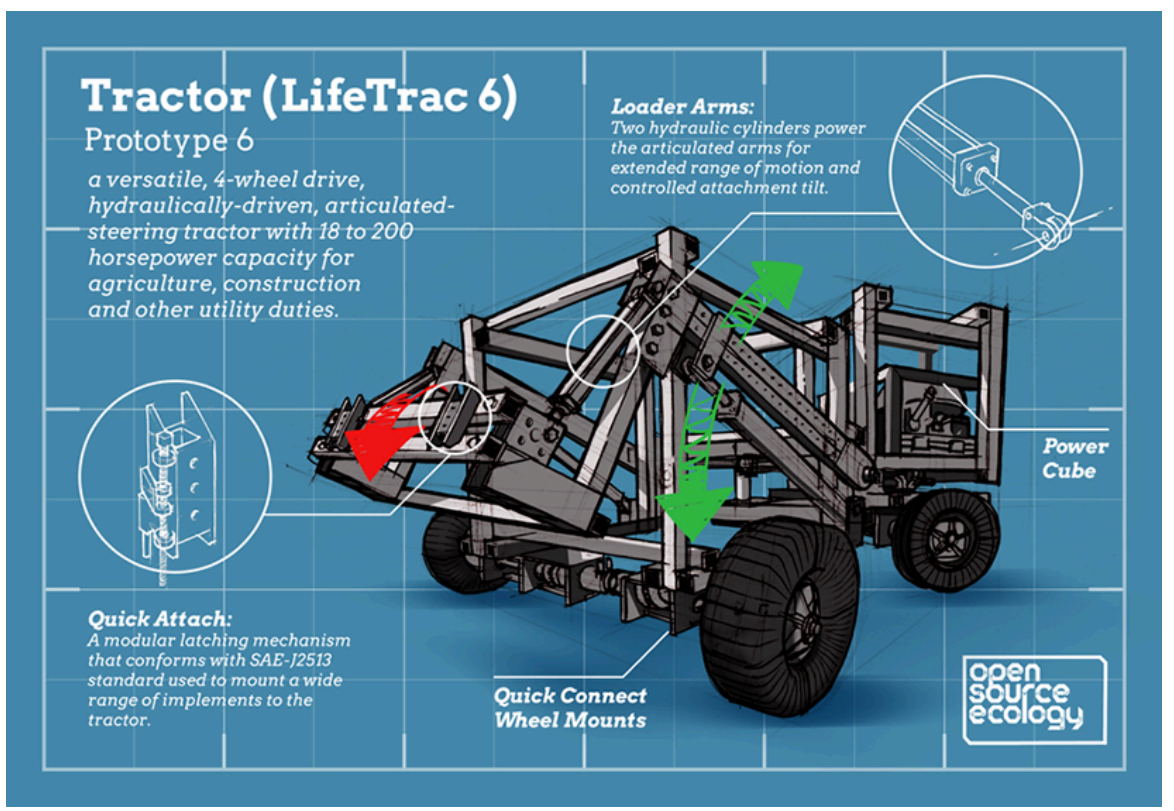




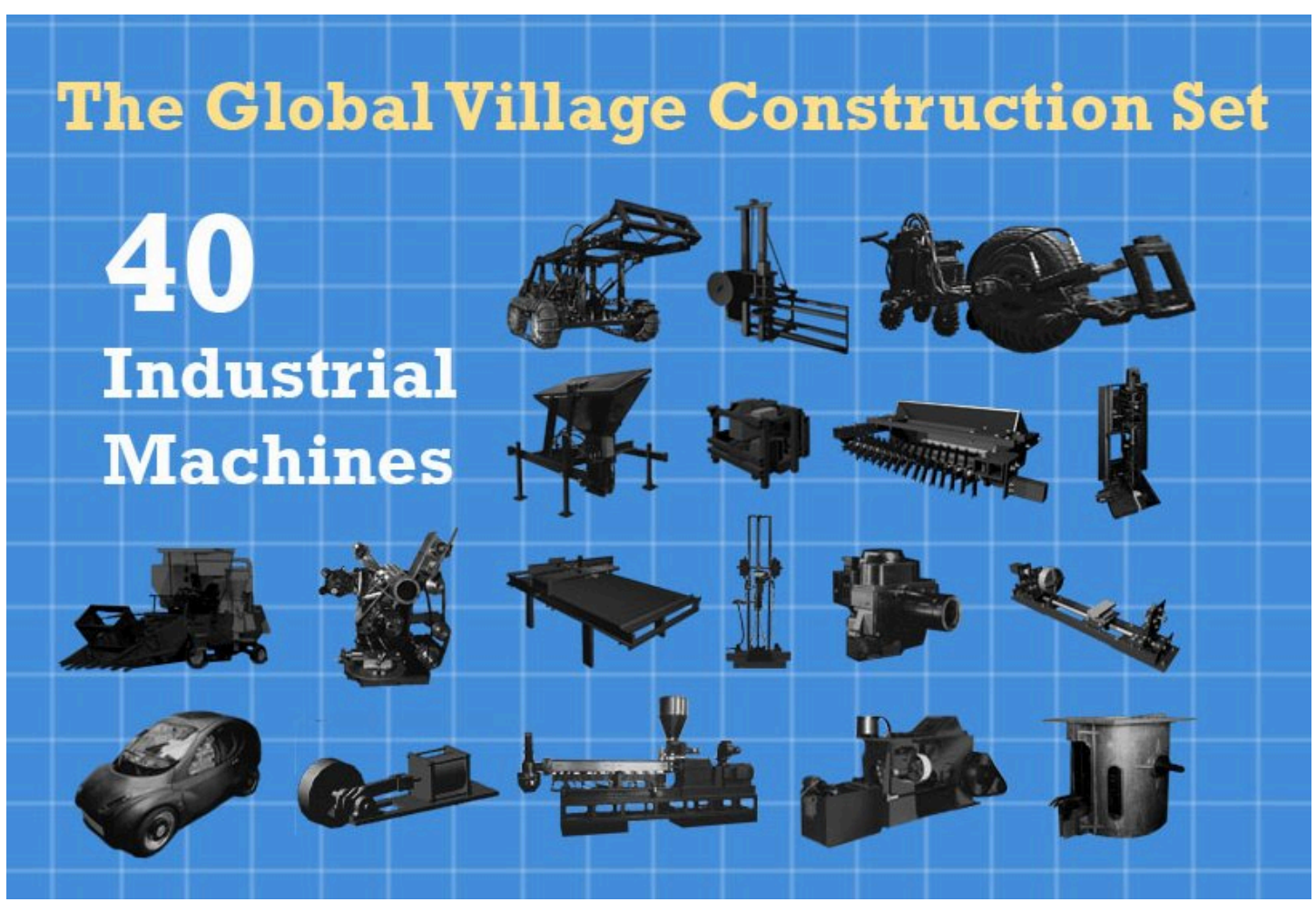

[4.5] Tractor and Global Village Construction Set from OSE (OSE, 2016a and 2016b)

Open Source Ecology is an international collaboration of people "who come together in different configurations for development opportunities, including design sprints and workshops" (Gibb, 2015: 134).

Originally founded in the USA, the OSE network is now spreading into different countries (Bulgaria, Germany, Greece, Italy, Spain, UK), where preparations are made, people come together and start building these machines to sustain their lives. This project goes beyond the successful OSH projects mentioned previously. It is not limited to one specific product or machine. It touches of the very fabric of how people live on this planet. Open Source Ecology published the Open Source Ecology Paradigm, which presents a number of relevant statements explored further in Chapter 4.3 - Personal Motivation and Meaning.

This overview of open source hardware projects serves as the basis for the following exploration into the characteristics of open source projects. 


\subsection{Characteristics of Open Source Projects}

A human being is part of the Whole...He experiences himself, his thoughts and feelings, as something separated from the rest... a kind of optical delusion of his consciousness. This delusion is a kind of prison for us, restricting us to our personal desires and to affection for a few persons nearest us. Our task must be to free ourselves from this prison by widening our circle of compassion to embrace all living creatures and the whole of nature in its beauty. Nobody is able to achieve this completely, but the striving for such achievement is, in itself, a part of the liberation and a foundation for inner security. -- Albert Einstein (1950)

This chapter will depict some main principles of open source projects and how they work. This covers theoretical backgrounds of networks, living systems, self-organization, motivation, creativity, striving for competence and empowerment. All these approaches of open source fit in an organic living paradigm and are the opposite of top-down and mechanistic models. In this chapter, there will be subchapters for dynamic living systems, the concept of strange attractor, networks, new media, creativity and self-organization.

\subsubsection{Dynamic Living Systems}

What is the difference between a living and a dead organism? This trivial question is very difficult to answer scientifically:

Speaking in terms of physics and chemistry, a living organism is aggregate of a great number of processes which, sufficient work and knowledge presupposed, can be defined by means of chemical formulas, mathematical equations, and laws of nature. These processes [...] are different in a living or a dead dog; but the laws of physics do not tell the difference, they are not interested in whether dogs are alive or dead (Bertalanffy, 1968: 141). 
Usually we do not find difficulties in distinguishing between a dead object and a living organism. The fundamental difference between a living and a dead system is that in a living system “innumerable chemical and physical processes are so 'ordered' as to allow the living system to persist, to grow, to develop, to reproduce, etc." (Bertalanffy, 1968: 139). The components of a living system are acting constantly, in parallel and permanently reacting to other components of the system. The overall behavior of the system is a result of the interaction of its individual components. This principle could also be seen in nature, where bird or fish swarms change direction literally at the same time. There is no visible order (meaning "command") from a leader of the community.

The primary order of the system "therefore, must lie in the process itself" (Bertalanffy, 1968: 141) and not in its physical and chemical composition. This order includes self-organization and regulatory feedback loops.

In the previous chapters, the idea of "Cartesian reductionism" was introduced. In this understanding, a machine is built from distinct parts and can be deduced to those parts without losing its machine-like character. For complex, living (real) systems this is not true "except under very special circumstances. Cartesian reductionism does not work for complex systems; it only reduces them to simple mechanisms" (Mikulecky n.d.).

It is very important to recognize that living systems are open, self-organized and interact with their environment (Bertalanffy, 1968: 141). This interaction with the environment occurs by means of information, matter and energy exchange. In the healthy system - an individual, a society, or an ecosystem - there is a balance between integration and self-assertion. This balance is not static but consists of a "dynamic interplay between the two complementary tendencies, which makes the whole system flexible and open to change" (Capra, 1982: 27). Furthermore, a living organism consists of a continual flux of matter, while its form is 
maintained (Capra, 1996: 18). In contrast, many scientific disciplines work only in and predefine a system as closed. Jeremy Rifkin (1980: 53) describes this approach in thermodynamics:

The reason scientists had such a hard time figuring out how living systems fit into the second law is because equilibrium thermodynamics is concerned with closed systems - systems in which energy and not matter can be exchanged with the outside surroundings. Living systems however, are open systems. Both matter and energy are exchanged with the outside. Living systems can never obtain an equilibrium state, while they are alive, because an equilibrium state means death.

Animals, people, organizations and communities are living systems. A tornado is perhaps a good example of a living system. A tornado looks like a stable structure form the outside, but it is actually a constantly moving system, permanently renewing itself. Particles are constantly moving in and out. The tornado is only stable as long as it is in movement. When the movement stops, it loses momentum and the tornado disappears and dies: "According to the systemic understanding of life, living Systems continually create, or re-create, themselves by transforming or replacing their components. They undergo continual structural changes while preserving their web-like patterns of organization" (Capra, 2002: 100).

A tornado is a nonlinear system away from static equilibrium. The equilibrium itself is dynamic, comparable to a horizon. It is always there, but can never be reached. This dynamic equilibrium is also called 'strange attractor', a concept that will be covered in the following chapter. In a tornado, there is a strange attractor, and there are also positive and negative feedback loops, expanding and stabilizing the whole system (Robert, 2011). Ludwig von Bertalanffy, one of the founders of general systems theory, explains that every living system is essentially an open system. Furthermore: 
It maintains itself in a continuous inflow and outflow, a building up and breaking down of components, never being, so long as it is alive, in a state of chemical and thermodynamic equilibrium but maintained in a so called steady state, which is distinct from the latter. This is the very essence of that fundamental phenomenon of life which is called metabolism (Bertalanffy, 1968: 39)

Open source projects tend to be chaotic and naturally in constant movement. Since there is no hierarchy, everyone is connected with everyone as in a chaotic system: "In a chaotic system, everything is connected, through negative and positive feedback, to everything else" (Briggs and Peat, 2000:34). People gradually fade when there is no activity. This is closely connected to the contribution of files to a software project. If there is no movement it might become boring and people might stop working on this project. There are many examples of open source projects that just fade away. But there is also the phenomenon that open source projects "reactivate" themselves: "It is fascinating to see how the social space of a collaborative software development project ebbs and flows over time" (Social Media Workgroup, 2011).

It is only when open source projects find the right balance between expansion and inclusion of their contributors that they are able to move on. If there is no structure at all, the community expands and it runs the risk of becoming too vague and losing its attraction. The same is valid for the opposite end of the spectrum, when the structure is too rigid. There are many examples where open source projects just stopped, and people went elsewhere to develop something else. Therefore, the dynamic equilibrium and its constant movement represent an important condition for an open source project. If there is no movement or openness to new inputs, the system turns into a closed system and the project will disappear. People lose interest. The constant movement and openness makes it stable and robust, just like dynamic self-organized systems, or a tornado. 


\subsubsection{The Strange Attractor}

A strange attractor in an organizational setting can be described as a shared vision, a team process and information flow. The term strange attractor is used in chaos theory and in complexity theory: "Chaos theory will be used to describe nonlinear, chaotic systems that are homogenous in nature and tend to move toward strange attractors. Complexity theory will refer to heterogeneous complex adaptive systems that move toward one or more attractor patterns" (Gilstrap, 2005: 60).

The strange attractor is outside and inside the system at the same time, and is never static. It is constantly in movement, never stopping and never traveling the same path twice: "Strange attractors are reflected in patterns of behavior, that is, shapes in space or movements over time, which are never exactly repeated but are always similar to each other" (Gilstrap, 2005: 60). A strange attractor is also a common idea or value system. In organizations it could be a corporate identity (CI); it could be a person. Nelson Mandela, when he was arrested and in prison, is a good example (Robert, 2011).

The system and the attractor mutually influence each other and resonate with each other. A strange attractor does not have to necessarily be a leader. A leader does; a strange attractor is. A strange attractor is also not necessarily limited to a person or an idea. In most cases, it is a combination of both or more (Robert, 2011). It is not necessary to have a leader in an organization. Rather, it could also work with the rotating principle we see in nature, particularly among birds or fish going in the same direction; one is leading, but this position is constantly changing. The strange attractor is not limited to one person. The strange attractor can be seen in movement likes "Al-Qaeda", “Anonymous” or "Occupy Wall Street”, where it is just an idea or common ground with which people resonate (Robert, 2011). 


\subsubsection{New Communication Technology and Networks}

It seems that new modes of communication, like Internet and mobile phones, enable us to share and exchange information very fast, thereby influencing organizational structures and the division of labor in a profound way. Reflecting that sentiment, Gibb (2015: 150) writes: "The rise of the Internet has allowed for rapid sourcing of tools for prototyping, designing, and manufacturing". Human networks can be seen as self-organized structures with the presence of an attractor. They could also be seen as (collective) living beings. These networks self-generate their own borders and interact multi-dimensionally. From a mechanistic perspective, this also includes actions that could be described as senseless; senseless in that they do not generate a measurable output. There will be some examples of this provided in the chapter on self-organization.

Living systems interact with the environment in a selective way to find and keep their dynamic equilibrium, a process which could be described as an open system with operational closures. These networks exist in every field, but are especially visible in open source software communities. Molly Beutz (2006) writes the following about network activism:

The same technologies that groups of ordinary citizens are using to write operating systems and encyclopedias are fostering a quiet revolution in another area-social activism. On websites such as Avaaz.org and Wikipedia, citizens are forming groups to report on human rights violations and organize email-writing campaigns, activities formerly the prerogative of professionals.

Human networks have intensified in number and geographical constellation since the Internet and new media created the possibility to connect more easily. Social networks are global networks beyond artificial borders and time zones; they are networks of communication. Through this shared context of meaning, individuals acquire identities as members of the social network, and in this way, the network generates its own boundaries. It is not a physical 
boundary but a boundary of expectations, of confidentiality and of loyalty, which is continually maintained and renegotiated by the network itself: "It is about making use of the vast opportunities of digital media and the Internet. Through sharing information and digital resources openly, there is great potential to make gains in innovation, distribution, reputation and marketing, collaboration and increase of efficiency and effectiveness" (Open it Agency, n.d.).

These new communication technologies are a precondition for the creation of centralized online knowledge hubs in the form of wikis and forums, which "allows for information exchange among people in remote locations, rapid distribution of design files, and a place to get questions answered fast" (Gibb, 2015: 150). Therefore, the reaction time in open source software communities is impressively fast. If there is a bug report (something not working as expected), it is, for the most part, resolved in a very short time. There is no organizational hierarchy to go through. As soon as a bug is reported, everyone is able to see the problem, there is no time delay, and everyone is invited to solve it. This method of collaboration is only possible through the communication technologies mentioned, which have been available for only a few years. This goes beyond nation-states, continents and cultures, since open source communities act mostly on a global scale.

A precondition for making this happen is transparency. It is not only necessary that the source code is open and the designs are published; it is also very important to keep track of changes to the software and document them in a transparent way. For that reason, there are several websites (e.g. sourceforge.net, github.com) offering exactly this type of service. Github.com is a web-based hosting service, including a control system for software versions: "Git is an extremely fast, efficient, distributed version control system ideal for the collaborative development of software. GitHub is the best way to collaborate with others. Fork, send pull requests and manage all your public and private git repositories" (Github, 2011). This way 
of working enables the contributors to be decentralized in their geographical locations. Everyone has the same access to the source of the software at the same time. If someone is attached to a bug, problem, product, etc., everyone is able to see it, be aware of the status of its development, and support co-creation and improvement. Similar ideas are starting to emerge in the open source hardware movement, as well, but are not ready at the moment.

\subsubsection{Innovation, Diversity and Creativity}

In practice, collaboration on a problem, question or topic is done in small working groups. In software development, bugs are published and then assigned to someone who is interested in the topic. Generally, this person is then supported by other people to solve the problem. These groups are often no bigger than a few members, and the composition of these groups changes from bug to bug, or from project to project.

What also happens many times is that someone starts to create a completely new module and functionality based on need, and then people jump on this train to support that person, because they might need the same end product, or they might just have fun doing it. None of this is predictable, and it is not controlled by anyone. There is no given intention of which direction the development has to go. Everyone participating on a project has his/her own intention. This way of working fosters creativity by its very nature.

To give an example of the complexity and diversity of open development, the comparison of Windows and Linux is relevant (Minichinelli, 2011). The following Figures 4.6 and 4.7 depict the timeline of the development of these two operating systems. The first figure portrays the innovation process on a closed, hierarchical, proprietary, linear software project. The second figure shows the innovation and diversity of an open, non-hierarchical software project. If we compare the timelines of the two operating systems until 2003, we see that the 
openness provokes diversity and results in creativity, which in the end bears a range of specific and appropriate technical solutions.

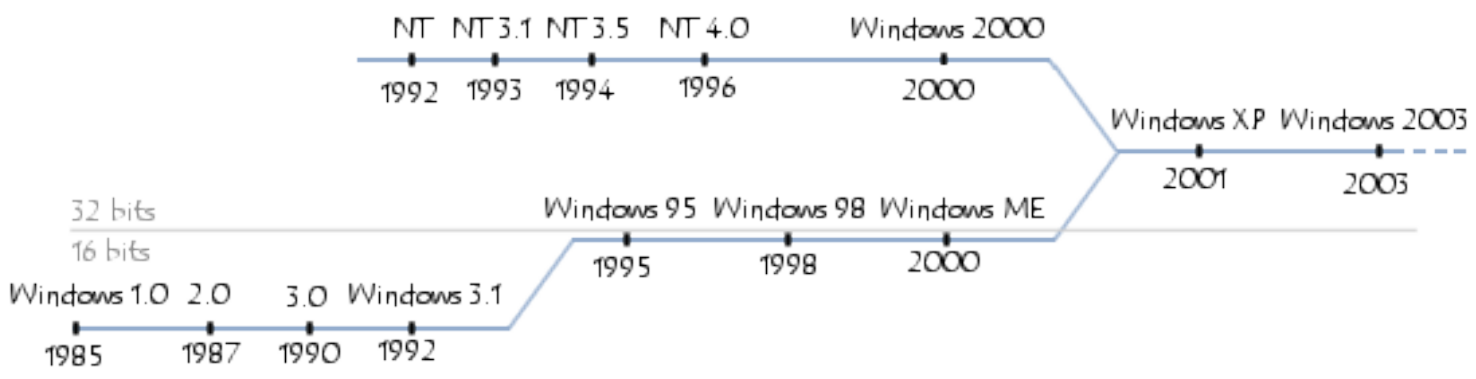

[4.6] Windows Family Tree (Nixcraft, n.d.)

It is also noticeable here that the formation is unpredictable, and that there are branches that stopped, known as "dead ends". In these cases, the interest and necessity of the technological solution was not appropriate anymore and the project was abandoned.

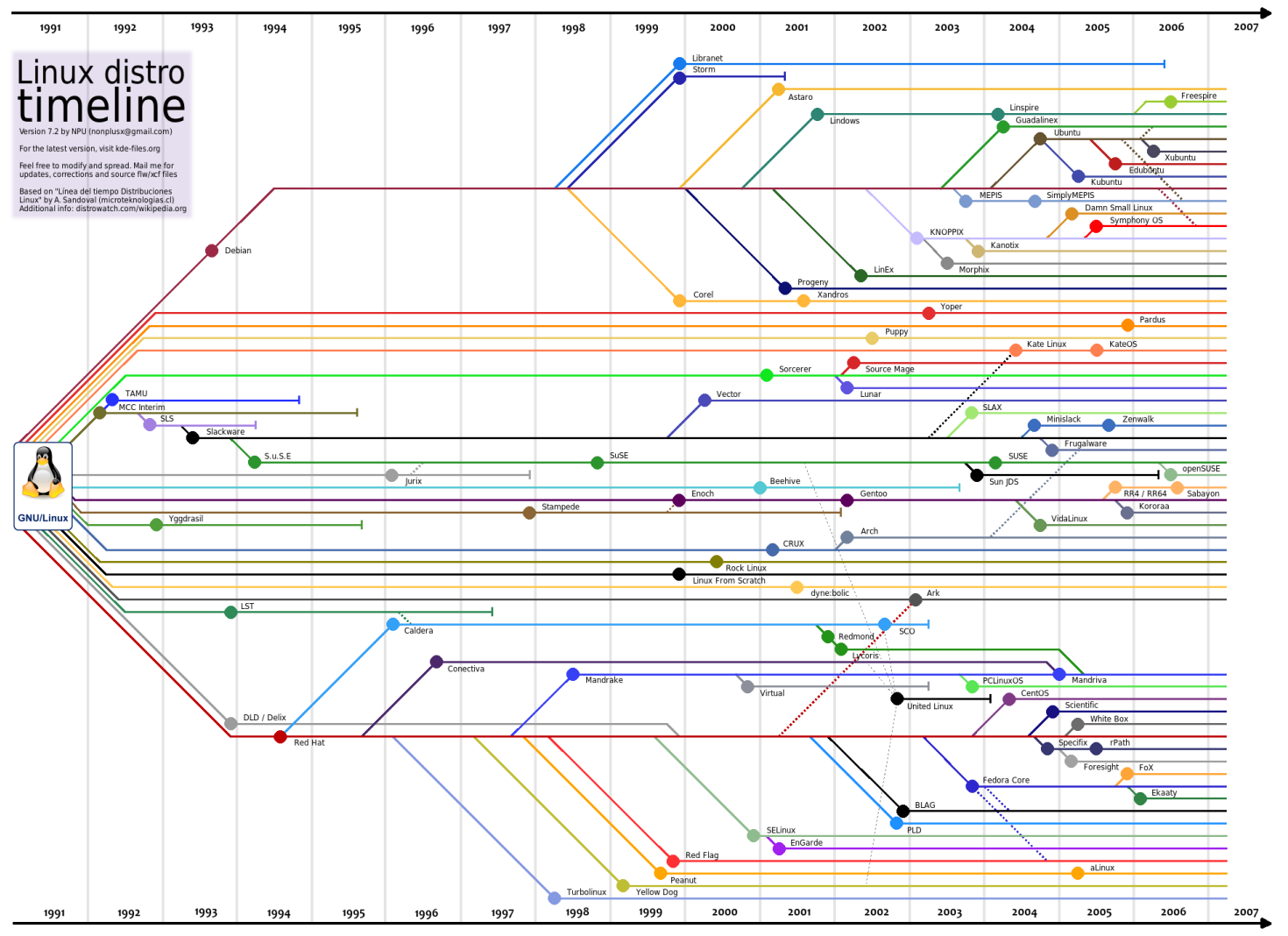

[4.7] Linux distribution timeline (Kioskea, n.d.) 


\subsubsection{Self-Organization and Do-Ocracy}

The essence of life is self-organization. A living system is self-maintaining, self-renewing, self-transcending.

Most organizations are set up in a linear, top-down manner, which is basically a way of functioning aligned with Newtonian Physics. Nothing moves until there is an impulse from the outside. Action is based on rational interest only, excluding feelings and emotions. No one in the hierarchical organization acts until there is an order from the top, from the boss of the organization. It is a clear linear structure and works through the principle of action and reaction.

If we look at the development work sector and at how the business sector is organized, we find top-down implementation at several points. Therein lies a common problem of development work. Donors exert a lot of pressure on development projects to achieve measurable results. The issue of the implementation process is a crucial point, as implementation mostly implies installing or introducing something from the outside, which can, to some extent, mean imposing something on someone. Conventional implementation approaches are mostly top-down in hierarchical structures. Michael Maren (1997) writes about his experience as a development worker and his personal benefits of reporting increasing numbers every year, while building things that were neither needed nor beneficial for anyone. Many other authors have written about this topic in a similar manner, and these observations are also visible in the business sector. In his book, The Hidden Connections, Fritjof Carpa argues that our hierarchical structure of organizations actually opposes a living system and describes a deep crisis of management and organization (Capra, 2002: 97). One of the main problems here is the top-down approach and its disconnection or alienation from 
life. Arturo Escobar goes a step further and states in his book, The Making and Unmaking of the Third World:

One of the many changes that occurred in the early post-World War II period was the "discovery" of mass poverty in Asia, Africa and Latin America. Relatively inconspicuous and seemingly logical, this discovery was to provide the anchor for an important restructuring of global culture and political economy. The discourse of war was displaced into the social domain and the new geographical terrain: the Third World (Escobar, 1995: 12).

The discourse on "if and how development work is legitimate" will not be answered here. However, it is important to mention, that the organizational structure of organizations combined with the "top-down" approach is not very successful most of the time. Intrinsic motivation is missing in this approach and self-organization is hardly happening.

One definition of self-organization is: "Self-organization is the spontaneous, often seemingly purposeful formation of spatial, temporal, spatiotemporal structures or functions in systems composed of few or many components" (Scholarpedia, 2008). Self-organizing systems are adaptive and robust: "They can reconfigure themselves to changing demands and thus keep on functioning in spite of perturbations" (Scholarpedia, 2008). These definitions of dynamic systems and self-organization serve also to describe the concept of networks, as already mentioned.

In open source projects, there is no formal authority over members in the community; there is no classical hierarchy. There is a hierarchy of engagement and experience, but not an organizational hierarchy as found in modern organizational structures. People self-organize in small working groups or teams and organize their contribution to the whole, which is some cases is the development of a product. As we have seen, this way of working is much more 
flexible than in strict hierarchical structures. This methodology also fosters much more creativity and flexibility, as many examples in open source software development have proven. By looking at the characteristics of open source projects, it becomes quite obvious that there is a strong connection to dynamic systems.

Therefore, the key concept of open source is rather the opposite of a classical hierarchical organizational structure: it is bottom up, as non-hierarchical as possible and self-organized. There is no hierarchical structure measuring results. There is a results evaluation on the micro level. If the result is positive, it might help at other micro levels, as well. It is not imposed on others; it is more a suggested solution. It is only an example of a local solution, based on local conditions, which might help in other cases, as well.

Self-organization is present in many cases in nature and looks chaotic from the outside, and it is quite visible and obvious in open source projects. There are no set hierarchies, no rules, and no pre-determined frame. Nevertheless, the system organizes itself and gives itself a structure. This structure stays open and flexible and is automatically undergoing a process of development. When there is constant movement, a project can be considered active, alive and functioning. This means that people from the outside entering, people from the inside leaving, as well as ideas, files or hardware, for example, go in and go out with almost constant frequency. Peter Fein, an activist from Telecomix ${ }^{25}$ described the process as follows:

People, all kinds of people, hang around in these IRC channels and just talk about all kinds of topics. Till at one point someone comes and says: let's do X. And then some people join, others wait and observe, others are busy with something else etc. It is self-organized, has no structure or hierarchy and also no way of decision-making. This guy who shows up and says let's do x could be a 14-year-old script Kiddy,

\footnotetext{
${ }^{25}$ Telecomix is a sociocyphernetic telecommunist cluster of internet and data loving bots and people, always striving to protect and improve the internet and defend the free flow of data. Telecomix, just like the Internet, knows no borders, technological or territorial (Telecomix, n.d.).
} 
sitting in his bedroom. The term self-organization is probably only valid at the second place. We use the term adhocracy ${ }^{26}$ much more (Fein, 2011).

Telecomix has no rules: "Telecomix is an adhocracy - we don't have leaders. We are all leaders. If you have an idea, find some collaborators and go do it.” (Fein, 2011, El Rodeo). This form of organization, "adho-cracy or do-ocrazy" is characterized through selforganization and decentralized shared authority. You do not have to ask anyone for permission, as you would in a hierarchical structure. It is open for anyone to create, innovate, do and share. This movement provokes also a lot of input that might not lead to anything substantive. But the reality is, that it could eventually lead to something, and that prompts creativity, which makes the difference. Many successful products were developed out of this approach.

The operating system Android or Google $\mathrm{Maps}^{27}$ might serve as an example here. At Google, employees are able to work one day per week on something that might be useful for the company. They are absolutely free to define the topic they want to work on. Android and Google Maps are a result of this approach, and they are extremely successful. Google Maps is one of the most powerful map services in the world, and the operating system Android is the most used operating system on mobile devices.

\subsubsection{Personal Motivation and Meaning}

If motivation is not driven by money in open source arrangements, where does the energy come from? One of the main characteristics of open source projects is that they are not driven by money or profit. People collaborate, contribute and share because they are motivated for

\footnotetext{
${ }^{26}$ Adhocracy, in this case, refers to a type of organization that could be described as the opposite of bureaucratic; non-hierarchical and without a common decision-making process.

${ }^{27}$ Android is an operating system for mobile devices. Google Maps is a web based mapping service.
} 
other reasons. According to the study, "Working for Free? - Motivation of Participating in Open Source Projects" from the University of Southern California, the sources of motivation are complex (Hars, 2003). A mixture of internal and external motivation is found among all of the contributors, and composition of contributors is diverse. Students and hobby programmers are more internally motivated, while paid programmers are more motivated by selling related products and services. One of the key findings of this study is that the composition of contributors and their motivation to participate in open source projects is very diverse and non-homogenous. As a consequence, the open source movement can draw from a diverse set of motivations (Hars, 2003). However, all these motivations have something in common. There is no immediate external reward and people are passionate toward what they are doing.

"If we want to know what is wrong with capitalism we have to look at the history of motivation and cooperation," explains Michal Bauwens, the founder of the P2P Foundation, offering the following words as an answer:

Pre-modern societies before capitalism were based on force. A slave had to give everything and a servant had to give half or more of what he produced. The genius of capitalism is to say instead of forcing you, why don't we create mutual self-interests and we will just exchange equal value with each other. In a way, that is great progress, because we evolved from outside, negative motivation (fear), to positive motivation, but still from the outside" (Bauens, 2008; personal conversation at Open Knowledge Conference, Berlin).

In the capitalist system, crucial problems are still inherent. If you do not have the prospect of earning money, and if you do not have outside motivation, you do not do anything. This system is based on personal self-interest on an individual basis. As a result, nobody is motivated to look outside of their self. No one looks at pollution or common goods, which 
should be free and available for everyone. Nobody wants to do anything that is not paid, at least not on a long-term basis. In contrast to the above-mentioned approach, participants of open source projects are driven by intrinsic motivation. The following list of motivation offers an idea of what moves people to collaborate and co-create:

○ Irk / Passion (to bring the common idea to the next level)

- Meaning ("to do something useful")

- Broader Consciousness (to support others / environmental sustainability)

- Shared dream / vision

- Relatedness (personal identification)

- Empowerment ("I am able to do this on my own")

$\circ$ Striving for Competence (Fame inside and outside the community)

Open Source Ecology serves as a good example, an organization that aims "to develop accessible blueprints for industrial and agricultural machines, called the Global Village Construction Set" (Gibb, 2015: 134). The core idea is the accessibility of technology for decentralized, local manufacturing and sustainability.

\subsection{A Collaborative Society? - Some Observations}

This chapter offers conclusions on the approach of OS and depicts a number of examples in society that are linked to the ideas of collaboration, sharing and sustainability. This can be seen as a shift in values, toward a post-capitalist society, with the intention of sustainability. This chapter covers the idea of Appropriate Technology (AT), Peer Production, Distributed Enterprises, 3-D Printing, and Hackerspaces, while at the same time offering comments on the transition to renewable energy. The importance of renewable energy is described in the chapter on industrial revolutions, which explores how communication technology and the use and distribution of energy define our economic model and organizational structures. 


\subsubsection{Third Industrial Revolution and Collaborative Economy}

A more distributed and collaborative industrial revolution, in turn, invariably leads to a more distributed sharing of the wealth generated.

-- Jeremy Rifkin (2011: 115)

Industrial revolutions and fundamental economic transformation occur "when new communication technology converges with new energy systems" (Rifkin, 2011: 35). According to Rifkin, organizational structures are based on communication technology: "The new forms of communication become the medium for organization and managing the more complex civilizations made possible by new sources of energy. The infrastructure that emerges annihilates time and shrinks space, connecting people and markets in more diverse economic relations" (Rifkin, 2011: 35). When put in place, economic activity advances and the living economy is based on the organic relationship between communications technologies and energy sources. To emphasize the importance of communication technology for the economic structure, Rifkin offers the following words:

Communication technology is the nervous system that overseas, coordinates, and manages the economic organism, and energy is the blood that circulates through the body politic, providing the nourishment to convert nature's endowment into goods and services to keep the economy alive and growing. Infrastructure is akin to a living system that brings increasing numbers of people together in more complex economic and social relationship (Rifkin, 2011: 35).

The first industrial revolution was based on the introduction of steam-powered technology into printing, which then became the primary communication tool. The steam printing machine greatly increased the speed of printing and significantly reduced the cost:

Print material, in the form of newspapers, magazines, and books, proliferated in America and Europe, encouraging mass literacy for the first time in history. The advent of public schooling on both continents between the 1830 s and 1890 s created a 
print-literate workforce to organize the complex operations of a coal-powered, steamdriven rail and factory economy (Rifkin, 2011: 35).

The first industrial revolution started with mass printing and the mechanization of the textile industry. The second industrial revolution took place when electrical communication converged with the oil-powered internal combustion engine in the first decade of the twentieth century: "The electrification of factories ushered in the area of mass-produced goods, the most important being the automobile. [...] Virtually overnight, millions of people began to trade in their horse and buggies for automobiles" (Rifkin, 2011: 35). With the moving assembly line, mass production began.

The third industrial revolution (TIR), which is already under way, is based on digital fabrication and the Internet. We have entered again into a process of convergence between communication technology and energy regimes: "The conjoining of Internet communication technology and renewable energies is giving rise to a Third Industrial Revolution” (Rifkin, 2011: 35). The TIR will reshape the way we transform and use energy, how we do business and organize. The TIR is organized around distributed renewable energies, which are available everywhere for free. These dispersed energies will be collected at millions of local sites and then bundled and shared with others over intelligent power networks (Rifkin, 2011: 115). It will change almost every aspect of our lives. Rifkin (2011: 35) shares the example of music and knowledge sharing and how it affected the traditional economic powers, as follows:

The music companies didn't understand distributed power until millions of young people began sharing music online, and corporate revenues tumbled in less than a decade. Encyclopedia Britannica did not appreciate the distributed and collaborative power that made Wikipedia the leading reference source in the world. 
Thirty years ago, the very idea that hundreds of thousands of professional and amateur scholars from every part of the world would collaborate and co-create academic and popular essays on virtually every conceivable topic in every discipline, without any payment, and make the information available to everyone, would have been unthinkable. Today, the English version of Wikipedia offers more than 5.2 billion articles and continues to grow at a rate of around 20,000 articles per month. If the English version of Wikipedia would be printed (without images and other multimedia content) in book form with a format similar to Encyclopedia Britannica, it would be 2319 volumes ${ }^{28}$. Wikipedia is the sixth most visited site in the Internet ${ }^{29}$.

There are many more examples, previously mentioned in part, where traditional business models and established industry branches clash with new ideas made possible through new communication technologies and established networks of individuals. This shift from markets to networks includes also a different business orientation, as Jeremy Rifkin (2011: 116) notes:

The adversarial relationship between sellers and buyers is replaced by a collective relationship between suppliers and users. Self-interest is subsumed by shared interest. Proprietary information is eclipsed by a new emphasis on openness and collective trust. The new focus on transparency over secrecy is based on the premise that adding value to the network doesn't depreciate one's own stock but, rather, appreciates everyone's holdings as equal nodes in a common endeavor. In industry after industry, networks are competing with markets, and open source commons are challenging proprietary business operations.

This shift in one's intention has already been explored in depth in the previous chapters on motivation and the open source approach. At this point, the study will elaborate on the specific technologies that are making the third industrial revolution possible.

\footnotetext{
${ }^{28}$ Statistics retrieved September 12, 2016 from https://en.wikipedia.org/wiki/Wikipedia:Size_of_Wikipedia ${ }^{29}$ Page Rank retrieved September 12, 2016 from http://www.alexa.com/siteinfo/wikipedia.org
} 


\section{D Printing}

In 1973, Ernst Friedrich Schumacher was asked the following question: "What is it that we really require from the scientists and technologists?" He answered: "We need methods and equipment which are:

- cheap enough so that they are accessible to virtually everyone;

- suitable for small-scale application; and

- compatible with man's need for creativity

Out of these three characteristics is born non-violence and a relationship of man to nature which guarantees permanence" (Schumacher, 1973: 21). Forty years later we have one technology "which guarantees permanence", and it is called 3D printing.

With a 3D printer, a product can be virtually designed and printed to create a solid object by building up successive layers of material. In the last few years, 3D printing has exploded into the mainstream and is transforming the way we design, manufacture, and repair objects. Today, 3D printers are in use in scientific research labs, manufacturing facilities, classrooms and universities, and your local community hackerspaces and FabLabs. Hackerspaces (sometimes called makerspaces) are collective spaces for people who are interested in experimenting with art, technology and science. In hackerspaces, space, tools and knowledge and experience are shared: "Many hackerspaces teach classes and have open hack nights for the public to come learn some tricks of many different trades" (Gibb, 2015: xv). FabLabs (Fabrication Laboratory) are similar to hackerspaces with the difference that they focus on digital fabrication: "From TechShop and Langton Labs in San Francisco to iHub in Nairobi, there is now an emergence of community hacker and maker spaces in urban areas around the world that utilize open source tools" (Thomson \& Jakubowski, 2012: 64). Open source hardware, DIY and 3D printing are closely connected: 
What you may not have seen in the $3 D$ printer media rush is how $3 D$ printing is spreading the use and creation of open source hardware. Not only are many of the desktop $3 D$ printer in use today based on open source hardware designs, but they also greatly facilitate the creation of new open source hardware (Gibb, 2015:94).

Local manufacturing and global development is possible. With the Internet and open access to online information, along with high-speed communication, designs can be adapted to local needs and improved from anywhere in the world. In combination with the local transformation of renewable energy and $3 \mathrm{D}$ printing, the costs of production will decrease significantly. In his book, The Zero Marginal Cost Society, Jeremy Rifkin also argues that the cost for transportation will decrease because renewable energy-fueled vehicles, unmanned and totally autonomous, will eliminate the costs of human labor and fuel. And the manufacturing of the transport vehicles will decrease because of local 3D printing, which is also driven by renewable energies. He calls it the "Zero Marginal Costs Society" and predicts an increase in efficiency of production with an increased awareness of the natural environment.

What all these developments have in common is collaboration, decentralization and trust. With trust I mean a form of empowerment and believe in oneself. Jeremy Rifkin goes a step further and also connects the decreasing interest in politics in Western democracies with the rise of the TIR and a shift towards collaboration. He notes:

In the coming era, both capitalism and socialism will lose their once-dominant hold over society, as a new generation increasingly identifies with Collaboratism. The young collaboratists are borrowing the principle virtues of both the capitalists and socialists, while eliminating the centralizing nature of both the free market and bureaucratic state (Rifkin, 2014: 23). 
Another example worth mentioning, aligned with the TIR and collaboration, is that of PeerProduction and Distributed Enterprise.

\subsubsection{Peer Production and Distributed Enterprise}

One of the most fateful errors of our age is the belief that the 'problem of production' has been solved

--- (Schumacher, 1979: 1)

Peer production is a way of co-creating and producing goods, services or ideas through a selforganized community of individuals who gather to produce a shared outcome. The term itself was first used in The Wealth Of Networks, a book published by Yochai Benkler in 2006. He argues that humans are equipped with the deep will to create, communicate and connect with other people. This, combined with the enormous potential of the workforce, as well as the whole range of diversity that humanity provides, holds significant potential for development:

A billion people in advanced economies may have between two billion and six billion spare hours among them, every day. In order to harness these billions of hours, it would take the whole workforce of almost 340,000 workers employed by the entire motion picture and re- cording industries in the United States put together, assuming each worker worked forty-hour weeks without taking a single vacation, for between three and eight and a half years! [...] They have diverse interests - as diverse as human culture itself. Some care about Viking ships, others about the integrity of voting machines. Some care about obscure music bands, others share a passion for baking. As Eben Moglen put it, "if you wrap the Internet around every person on the planet and spin the planet, software flows in the network. It's an emergent property of connected human minds that they create things for one another's pleasure and to conquer their uneasy sense of being too alone (Benkler, 2006: 55). 
Howard Rheingold (2005) gives an overview of the history of increasing wealth, stating that "cooperation; collective action and complex interdependencies play a more important role”. He argues that in the history of humanity, new forms of collective action have always increased wealth. By collaborating in hunting together, people were able to increase wealth in the form of protein. Through book printing and mass literacy, new forms of collective actions emerged in the fields of politics, physics and religion: Jefferson, Newton and Luther. The outcome was an increase of wealth. Now, a new form of cooperation, peer production, seems to create new forms of wealth: "Peer production describes a process by which many individuals, whose actions are coordinated neither by managers nor by price signals in the market, contribute to a joint effort that effectively produces a unit of information or culture" (Benkler, 2003: 1256).

Benkler defines peer production as radically decentralized collaborative production. This phenomenon is fostered by the shift from the industrial information economy to the networked information economy:

What we're seeing now is the emergence of a system of social sharing and exchange. Not that it's the first time that we do nice things to each other, or for each other, as social beings. We do it all the time. It's that it's the first time that it's having major economic impact (Benkler: 2003).

Peer production is only possible because of modern communication technology and is strongly connected to motivation. Michael Bauens (2008) described the advantages of peer production as follows: "The genius of peer-to-peer is that it filters out negative outside motivation, positive outside motivation and only stays with intrinsic motivation, voluntary passionate production". And thus, the project is designed so that individual interest in improving something corresponds with value for everybody, because everything you improve for yourself becomes part of the whole project, and the whole project is accessible for 
humanity as a whole. "Peer-to-peer is post-capitalistic because is it not about commodities, not about wage relationships, not about producing for the market (with commodities and exchange value), so if you do that then part of you is already out of the market" (Bauens, 2008 and personal conversation at Open Knowledge Conference 2011 in Berlin).

Peer production is also strongly related to innovation. In companies, innovation is mainly driven by competition. You want to innovate and improve, because you want to be better than the competitor. Unfortunately, financial advantage often lies behind this innovation process. If there is a monopoly, there is no competition.

In peer production, the intrinsic motivation of innovation is the driving force behind invention and productivity. People want to innovate, not just to be better than the other person, but they also want to permanently make the best possible product available. The grand scale advantage of this is that the resulting outcomes are shared with everyone. In this way, innovations and development are very fast and flexible.

According to the Open Source Ecology Paradigm (2011): "Global collaboration in open product and process design leads to best practices being commonly available. This is opposed to the dominant paradigm of today - where a few companies having the best products or monopoly control, and by definition, the rest is mediocre".

A precondition for reaching global peer production is transparency and openness. Collaborative development is only possible when the significant product information is available. If access to the technology blueprints and to outside participation in development is granted, a product can be seen as open source. A step further is the idea of a distributed enterprise, which additionally also gives access to the enterprise blueprints: "A distributive 
enterprise is a social enterprise that focuses on open economic development. More specifically, it is a transparent enterprise that promotes-at the core of its operational strategy - the capacity for others to replicate the enterprise without restrictions" (Thomson \& Jakubowski, 2012: 62). A traditional franchising system is similar to this approach; with the difference that it would be made open and replicable by everyone. The model of distributive enterprise is a highly human-centered mode of economics and collaborative production, "whereby citizens regain their autonomy in a complex world" (Thomson \& Jakubowski, 2012: 63).

In the OSE paradigm, the importance of reconnecting to nature is stated as follows:

Transparency of the connection between technology and nature means that people begin to respect nature. This happens when people begin to respect that their wellbeing comes from nature. This transparency is facilitated when economically productive activities happen as close to the community as possible - not out of sight, out of mind in remote locations. This is true environmental accountability - as one tends to not destroy their own environment. Thus, there is a direct connection between transparency of production to natural regeneration - as people begin to make more sound production choices - by understanding the connection of production to the land. This means that industry no longer needs to occur in the form of toxic wastelands - but instead - eco-industry, on a human scale - serving the needs of people, not centralized industries competing for world domination (Open Source Ecology, 2011).

Decentralization and local production are essential for awareness-building and a reconnection to nature. This is especially valid for energy supply and consumption . With decentralized energy supply, consumption is as close as possible to the source of energy production, so that people become aware again of what nature offers and where the limits are. If there is no forest, there is no water. If there is no water, there is no energy. A natural consequence, then, 
is that humans take care of the forest that will provide their necessary water in the dry season. This "depends on increasing the density of knowhow and technology in every community which comes from the open paradigm - open information, open communication, open everything” (Open Source Ecology, 2011).

During the Open Knowledge Conference 2011 in Berlin, I had a conversation with a conference participant, who described peer production and open economy in the following way:

I have talked to people from Luxemburg, Canada, South Korea, and from every other country I can think of. We are all doing this for exactly the same reason and we are all coming from completely different places, with different experiences and various backgrounds, but through collaboration we stimulate and push each other constantly to new limits and share our experience. This is what the world should be like.

\subsubsection{Appropriate Technology 2.0}

New forms of organization and society-oriented intentions might also be visible through Open Source Hardware and Appropriate Technology (AT). AT is an ideological movement, initiated in the late 1960s by the influential work of Dr. Ernst Friedrich Schumacher, "Small is Beautiful." Characteristics of AT solutions are typically small-scale, decentralized, locally controlled, environmentally friendly and people-centered. AT was the main approach in development work until the 1990s and was highly focused on needs, a bottom-up approach, in theory. Unfortunately, due to several reasons (e.g. acceptance and technological exchange) this approach lost its momentum, and the AT movement declined in recent decades.

Open Source Hardware combined with AT and new communication possibilities creates an environment I will call the AT 2.0 approach. Global design and local manufacturing are 
changing our intentions on design, production, use, and our relation towards nature and environment in a profound way, clearly visible with decentralized renewable energy solutions. This approach has the potential to provoke profound social change away from a linear, hierarchical, centralized system based on growth, towards a non-linear, self-organized, interconnected, decentralized system based on sustainability and existing in a more harmonious relationship with nature.

As a nuanced approach, AT 2.0 can be extended to all kinds of technological fields, like science and education, where OSH has been introduced through university and research laboratories. According to Joshua Pearce, Director of the Open Sustainability Technology Research Group at Michigan Technological University, open source hardware is lowering the cost of laboratory hardware to a fraction of what it was before. Experimental research is mostly done with purchased hardware equipment and custom single-built equipment, fabricated in-house. Pearce explains that distributed digital manufacturing of open source hardware will make science both cheaper and more accessible (Pearce,n.d., as cited in Mills, 2016): "In science, we all have this problem where we pay so much for scientific equipment that it overwhelms our budgets." In fact, a lot of equipment is simple-mechanically speaking - and can even be manufactured with a do-it-yourself 3D printer like the RepRap (Pearce,n.d., as cited in Mills, 2016).

\subsection{Conclusion and Transition to Renewable Energy}

It is quite impressive how the development of communication technology fosters means of collaboration, collectivism and sharing. Open source is a wonderful example for non-linear and non-mechanistic forms of self-organized collaboration and non-competitive sharing. Just like a tornado, an open source project is an unpredictable living system. One of the most 
interesting things is that this non-mechanistic approach of solving problems and developing high-tech products is actually able to develop mechanistic and highly technical solutions. The collective, lateral power of people, connected directly to each other is a totally new form of organizing, sharing, exchanging and problem-solving. The highly decentralized and autonomous approach is possible thanks to new information technology. According to Jeremy Rifkin (2011), industrial revolutions occur when new forms of communication meet with new forms of energy. It is obvious that there is a current shift from fossil fuels to renewable energy from the sun, which Jeremy Rifkin describes as democratic energy since it is renewable and therefore endless, free and accessible for everyone - in contrast to centralized fossil fuel and nuclear energy distribution.

Along with the Internet and communication technologies, highly decentralized renewable energy supply is among the driving factors for the Third Industrial Revolution. The next chapter will give an overview of physical concepts for renewable energy principles and solutions for rural electrification, potentially applicable in Global South and open source scenarios. This chapter provides the pretext for the feasibility study presented afterwards. 


\section{Renewable Energy and Rural Electrification}

This chapter outlines the necessity for rural electrification through renewable energy, and offers a number of technical options. First, I define energy consumption and energy access, describing the current situation as they exist today. Secondly, I offer an introduction on renewable energy, including comments on the environmental and their importance for the preservation of this planet. Then, I describe four different technical concepts of renewable energy transformation and offer an analysis of their positive and negative aspects for rural electrification. The focus here is firstly on the technical concept, including its basic physical background, because this is crucial for understanding the thesis and a precondition for Do-ItYourself (DIY) feasibility. The physical energy transformation processes will be explained in "excurse sections", which give a purely technical access to the these purely physical topics. In addition to the technical elements, I focus on the concepts of manufacturing, scalability and resource availability. The technology comparison table and feasibility matrix will be followed by a SWOT-Analysis ${ }^{30}$ of small-scale hydro power, with which I close the chapter.

\subsection{Population Growth, Energy Consumption and Electrical}

\section{Distribution}

This chapter offers an overview on energy consumption and electrical distribution worldwide. To some extent, this also includes historical and prospective outlooks on the development of energy consumption and electrification in the near future. The focus here is on the field of

${ }^{30}$ SWOT "Strengths Weaknesses Opportunities Threats Analysis" 
rural, remote-area electrification, which is particularly important in so-called low-income economy countries, where national electricity grids often do not cover remote areas.

\section{Energy Consumption:}

The following graph shows the composition of all forms of energy consumption in use (not only electricity), whereas this study focuses solely on renewable energy. As we can see, energy consumption has almost doubled from 1973 to 2012 and reached over 8979 Mtoe $^{31}$ in 2012, which is a growth rate of almost 93 per cent (more specifically, from 4672 Mtoe in 1973 to 8979 Mtoe in 2012). We can also see that energy consumption has significantly

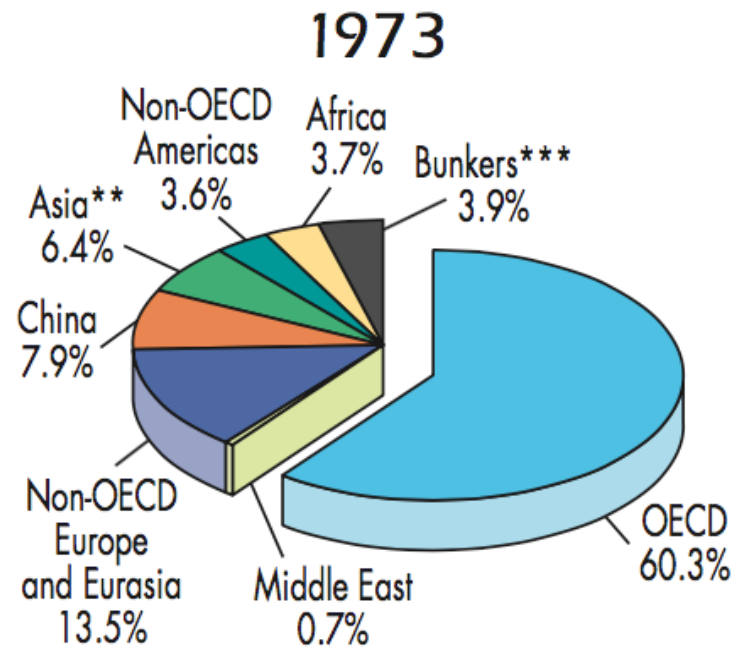

4672 Mtoe

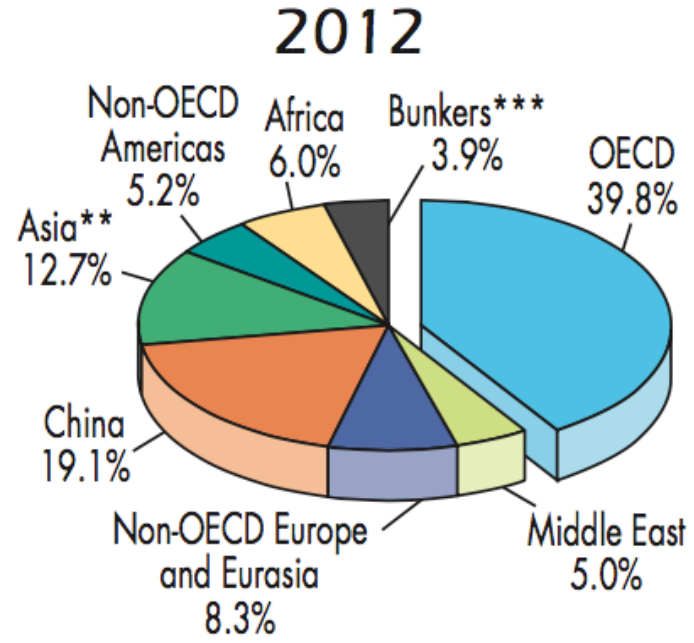

8979 Mtoe

[5.1] Energy total final consumption by area (IEA Energy Statistics, 2014: 30) **Asia exclude China

*** Includes international aviation and international marine bunkers

shifted to Asia, Africa and Non-OECD America ${ }^{32}$, whereas the consumption of OECD ${ }^{33}$ countries decreased. China almost tripled its consumption up to almost 20 percent in the

\footnotetext{
${ }^{31}$ Ton of oil equivalent (toe) is a physical unit equal to amount of energy released by burning one ton of crude oil. The amount of energy is approximately 42 Giga Joules

${ }^{32}$ America without Canada, Chile, Mexico and USA
} 
timeframe of 39 years. Africa and Non-OECD America behaved comparably and doubled their energy consumption with an increase up to roughly 12 per cent of world energy consumption. (IEA, 2014: 30). The most remarkable increase is evident in the Middle East, with consumption rising from 0.7 per cent in 1973, up to 5 per cent in 2012. At the same time, the energy consumption share of OECD countries decreased from over 60 per cent in 1973, to below 40 per cent in 2012 .

This statistic demonstrates the enormous global increase in energy consumption and an evident shift of energy consumption from higher-income countries to lower-income countries.

\section{Energy Consumption Per Capita:}

The variation of energy consumption by region is vast. In the graphs of figure 5.1 above, we see that in 2012 OECD countries still utilized roughly 40 per cent of the world's energy; China roughly 20 per cent. The population of OECD countries and China is almost the same. In 2012 roughly 1.358 billion people lived in China, whereas the population of the OECD countries was about 1.254 billion (IEA, 2014: 48). The population of the OECD countries is a little less than the population of China, whereas its energy consumption is double that of China. OECD countries and China consume 60 per cent of global energy while including 2.612 billion people, representing only 37 per cent of the global population.

The following map [Figure 4.2] depicts the imbalance of electricity consumption of the state of New York and Sub-Saharan Africa, which is roughly equivalent: "In other words, the 19.5 million inhabitants of Now York consume in a year roughly the same quantity of electricity, 40 TWh, as the 798 million people of Sub-Saharan Africa” (IEA, 2010: 11). Another

\footnotetext{
${ }^{33}$ OECD countries: Australia, Austria, Belgium, Canada, Chile, Czech Republic, Denmark, Estonia, Finland, France, Germany, Greece, Hungary, Ireland, Israel, Italy, Japan, Korea, Luxemburg, Mexico, Netherlands, New Zealand, Norway, Poland, Portugal, Slovak Republic, Slovenia, Spain, Sweden, Switzerland, Turkey, United Kingdom, United States
} 
example, the population of Africa, Asia (without China) and Non-OECD America is about 3.870 billion. That is 55 per cent of the world's population ( 7.037 billion) and only 22.2 per cent of the world's energy consumption.

With these convincing examples, it is obvious that energy consumption per capita differs drastically around the world. We see that energy consumption per capita is very different from region to region, and from country to country. Moreover, per capita energy consumption differences can be great within one country, as well, with less energy often consumed in rural areas, as we will see later.

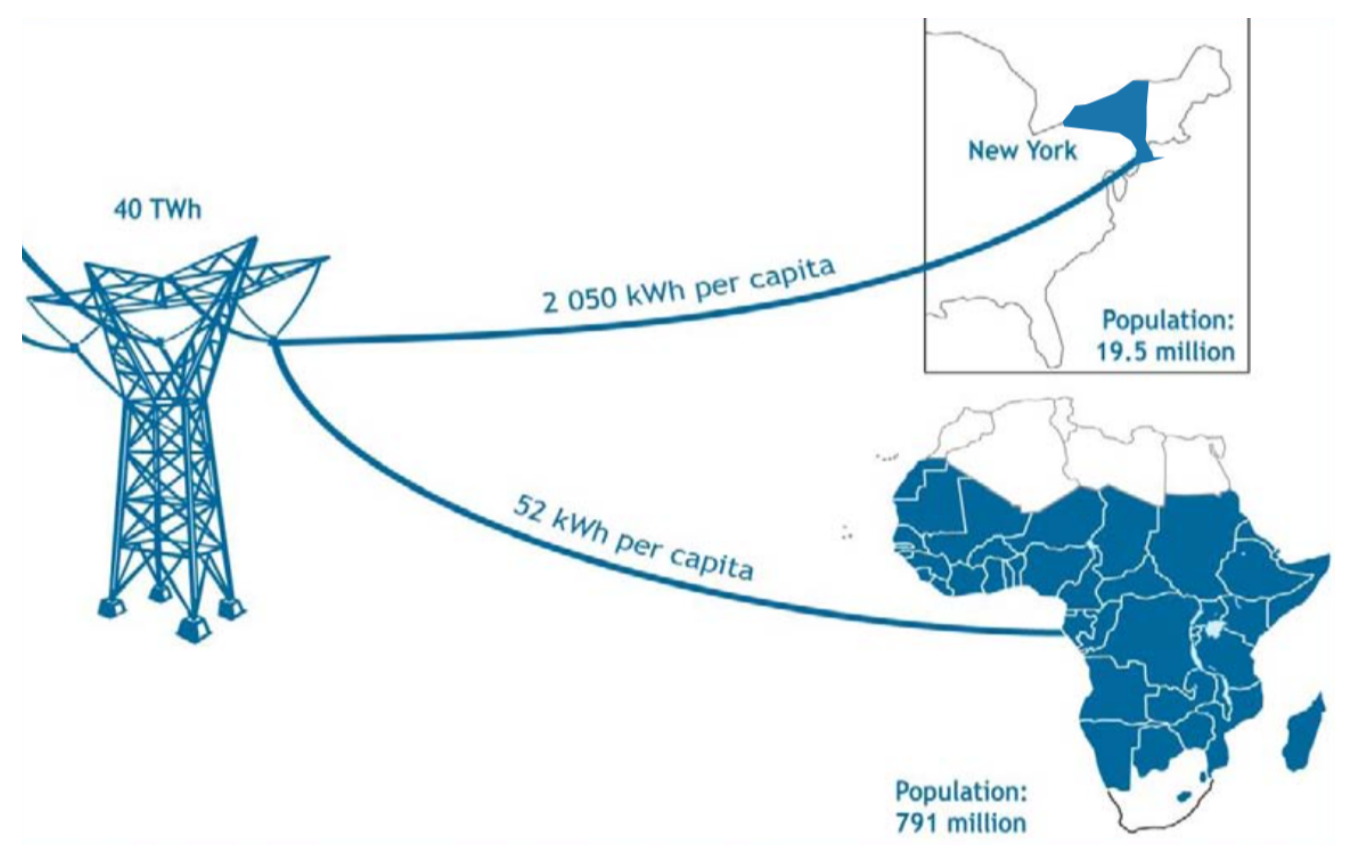

[5.2] Energy consumption in New York and Sub-Saharan Africa (IEA, 2010: 11)

\section{World Population and Population Growth:}

A tremendous change in population growth occurred with the Industrial Revolution, whereas it had taken all of human history until around 1800 for the world population to reach one billion (Population, 2015). The second billion was reached in only 30 years (1930), the third billion in less than 30 years (1959), the fourth billion in 15 years (1974), and the fifth billion in only 13 years (1987). In 207 years, the global population grew from 1 billion in 1804 up to 7 billion in 2011 (Population, 2015). During the 20th Century alone, the world population 
grew from 1.65 billion to 6 billion. The United Nations (UN) Population Division graph [Figure 5.3] demonstrates that in 1970 there were roughly half as many people in the world as there are now. UN Population Division projections imply that population growth will continue into the 21 st century, although more slowly (United Nations, 2004: 4).

The same graph also depicts the fact that population growth is mainly driven by Africa and Asia, whereas the population numbers of Europe and the Americas seem to stay almost stable or even decrease.

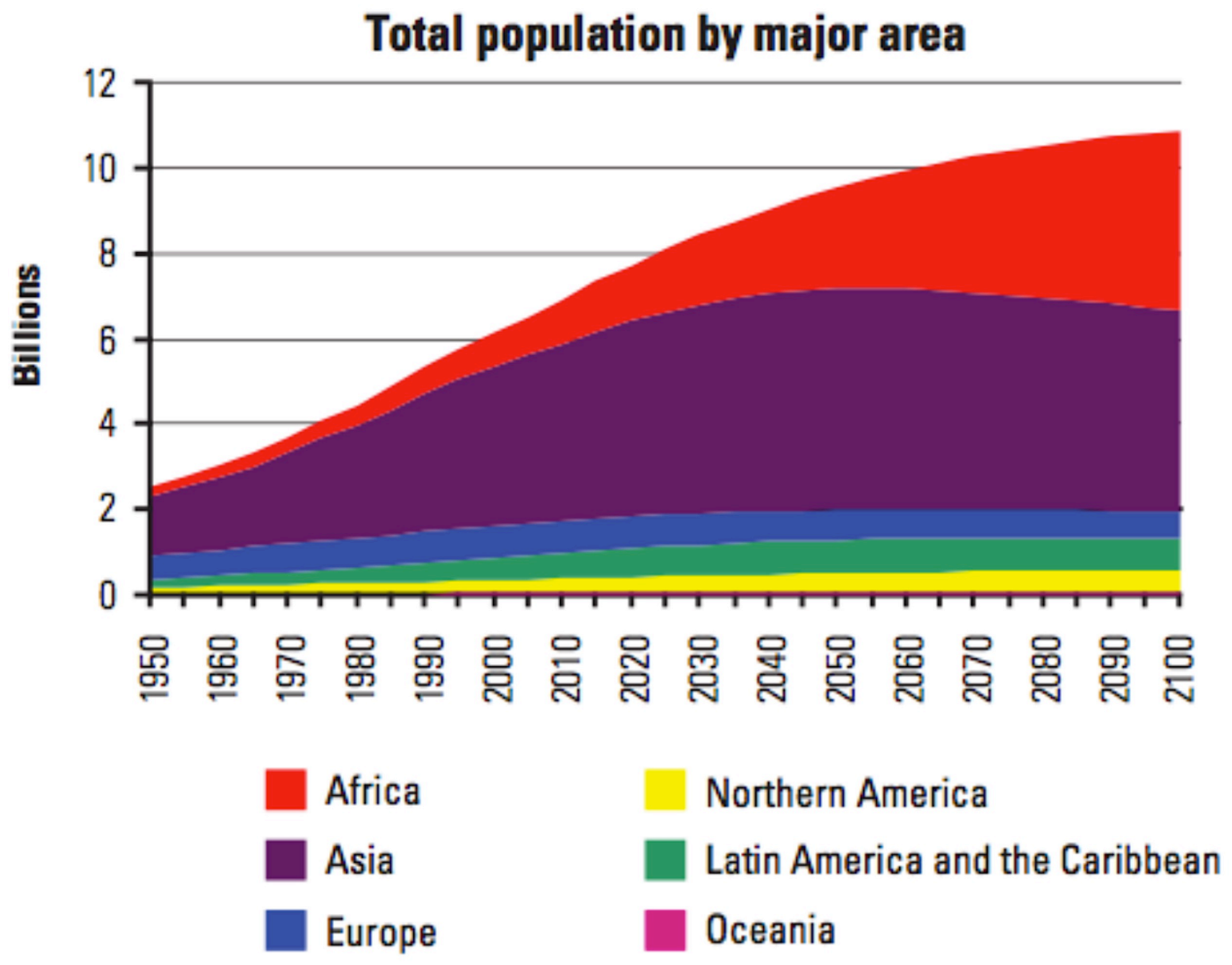

[5.3] World Population 2012 (UN Population Division, 2012: 2)

We have seen a global increase in energy consumption of about 93 per cent from 1972 to 2012. In the same timeframe, the global population grew from almost 4 to over 7 billion, reflecting a growth rate of 90 per cent. We have seen where population growth happens, and we have seen in which area energy consumption is shifting. It seems evident, then, that energy consumption growth corresponds with population growth. 
The following section explores the current state of energy access and distribution in the areas where population growth is happening.

\section{Energy Access and Distribution:}

The International Energy Agency $^{34}$ (IEA) estimates that in 2012, 1.3 billion people, a population equivalent to that of the entire $\mathrm{OECD}$, continue to live without access to electricity. This equals 18 per cent of the global population and 22 per cent of those living in developing countries (IEA, World Energy Outlook, 2014). The share of people lacking access to electricity differs significantly across regions, but is much greater in the least developed countries (LDCs) and sub-Saharan Africa than in other countries (United Nations Development Program, 2009: 11). Table 5.4 shows that the electrification rate of OECD and transition economies reaches almost 100 per cent, whereas the world average is at 82 per cent. The category of developing countries has an electrification rate of about 76 per cent. The share of people without electricity access for developing countries is visible in map 5.5 on the following page.

\begin{tabular}{|l|c|c|c|}
\cline { 2 - 4 } \multicolumn{1}{c|}{} & $\begin{array}{c}\text { Total Population } \\
\text { (in millions) }\end{array}$ & $\begin{array}{c}\text { Electrification } \\
\text { rates (\%) }\end{array}$ & $\begin{array}{c}\text { Total population without } \\
\text { electricity (in millions) }\end{array}$ \\
\hline World & 7.138 & 82 & 1.285 \\
\hline $\begin{array}{l}\text { OECD and transition } \\
\text { economies }\end{array}$ & 1.254 & $\begin{array}{c}\text { almost } \\
100\end{array}$ & 1 \\
\hline Developing Countries & 5.345 & 76 & 1.283 \\
\hline
\end{tabular}

[5.4] Electrification rate by economic power, (IEA, 2014)

The United Nations Development Program investigated and specified the location of where people without access to electricity actually live:

More than 80 percent of people without electricity access live either in sub-Saharan Africa or in South Asia. While sub-Saharan Africa makes up about 14 percent of the

\footnotetext{
${ }^{34}$ The IEA is an autonomous organization of 28 member states founded in response to the 1973 oil crisis, aiming to ensure reliable, affordable and clean energy. 
total population of developing countries, it accounts for almost 40 percent of the population without electricity access (UNDP, 2009: 11).

Access to electricity also varies dramatically among countries in the same region. For instance, in Latin America and the Caribbean, 62 per cent lack access in Haiti, but only 2 percent lack access in Brazil. In sub-Saharan African countries such as Chad, Liberia, and Burundi, more than 95 per cent of people lack electricity access, while the numbers are 25 per cent in South Africa, and less than 1 per cent in Mauritius (UNDP, 2009: 11). While, for instance, the electrification rate for Latin America reaches 95 per cent, there are still regions and countries like Haiti where the electrification rate is around 2 per cent. This unbalance is visible in figure 5.5 below, showing statistics for Africa.

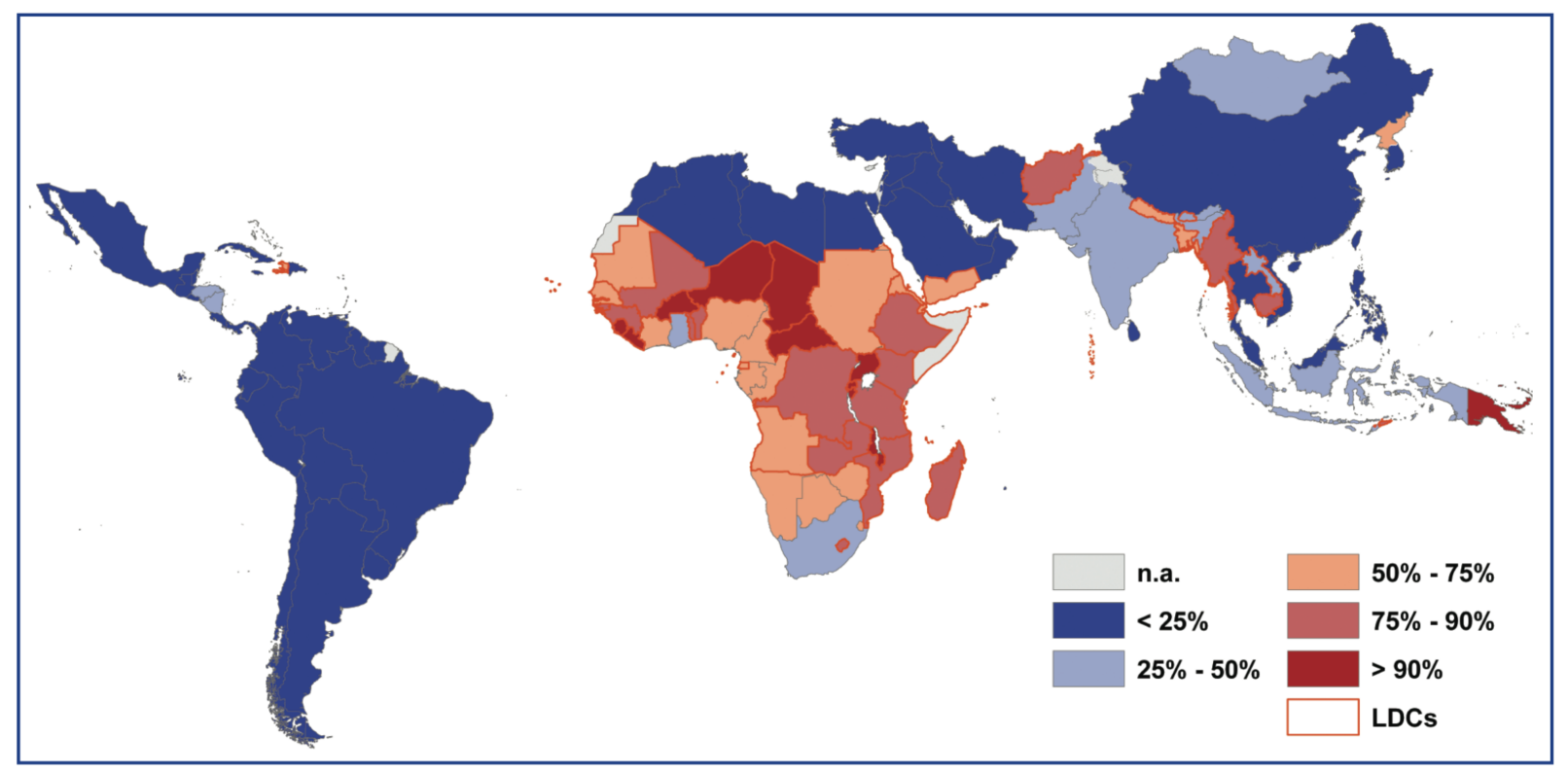

N.A. = not available.

[5.5] Share of people without access to electricity in developing countries (UNDP, 2009: 11)

The following table 5.6 gives an overview of absolute numbers of people lacking access to electrical power. In total Africa it is around 622 million people whereas in Sub-Saharan Africa it is 621 million people. This shows the different access of different regions. Similarly, the differences within a country can also be dramatic, as we see when we compare urban and rural electrification rates. In Latin America, for instance, the urban electrification rate is 99 
per cent, whereas rural electrification is at only 82 per cent. The most remarkable gap between urban and rural electrification lies in Sub-Saharan Africa (almost 60 per cent urban electrification and below 20 per cent rural electrification).

\begin{tabular}{|l|c|c|c|}
\cline { 2 - 4 } \multicolumn{1}{c|}{} & $\begin{array}{c}\text { Total Population } \\
\text { (in millions) }\end{array}$ & $\begin{array}{c}\text { Electrification } \\
\text { rates (\%) }\end{array}$ & $\begin{array}{c}\text { Total population without } \\
\text { electricity (in millions) }\end{array}$ \\
\hline \hline Africa & 1.091 & 43 & 622 \\
\hline North Africa & 178 & 99 & 1 \\
\hline Sub-Saharan Africa & 913 & 32 & 621 \\
\hline Developing Asia & 3.647 & 83 & 620 \\
\hline China & 1.358 & 100 & 3 \\
\hline India & 1.216 & 75 & 304 \\
\hline Latin America & 460 & 95 & 23 \\
\hline Middle East & 225 & 92 & 18 \\
\hline
\end{tabular}

[5.6] Electrification rate by region (IEA, 2014)

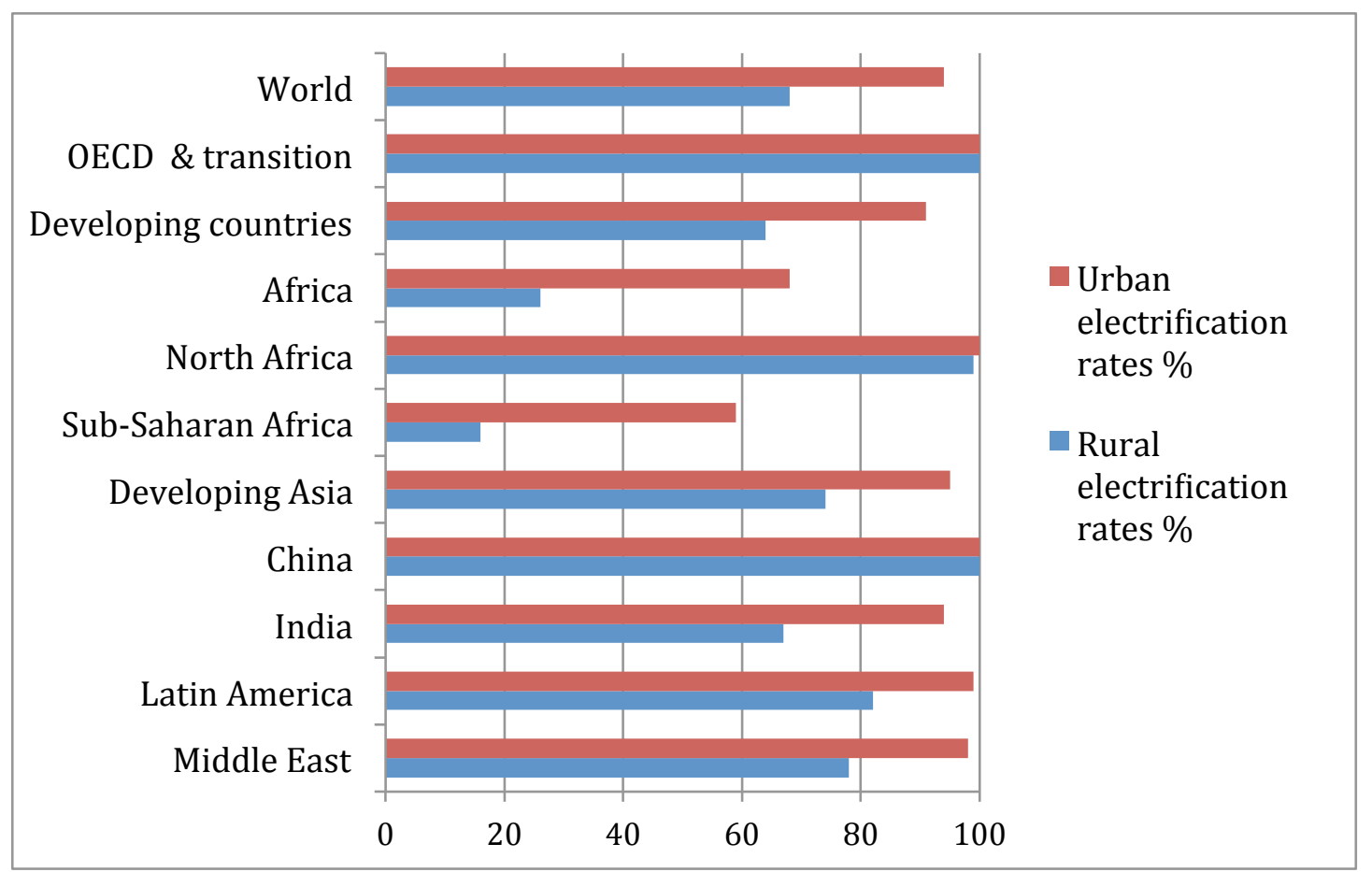

[5.7] Urban und rural electrification rates (author's illustration, numbers from IEA, 2014)

Graph 5.7 gives an overview of urban versus rural electrification. The world's urban electrification rate is at 94 per cent while rural electrification stands at 68 per cent. In most regions, the urban and rural electrification rates differ more than 10 per cent. The most obvious difference is visible in Sub-Saharan Africa, where only 16 per cent of the rural 
population has access to electrical power: "The lack of electricity access is much more severe in rural areas of LDCs and sub-Saharan Africa (87 percent and 89 percent, respectively, lack access) than in developing countries in general (41 percent lack access)” (UNDP, 2009: 11).

Within countries this imbalance is even more evident. The following chart 5.8 visualizes the discrepancy of rural versus urban electrification in selected countries. Senegal, for instance, has an electrification rate of around 90 per cent in urban areas and only 55 per cent nationwide. However, only about 28 per cent of the rural population has access to electricity. In Kenya, only 7 per cent of the rural population is connected to electrical energy, whereas the national electricity supply is available for 60 per cent of the country's urban population.

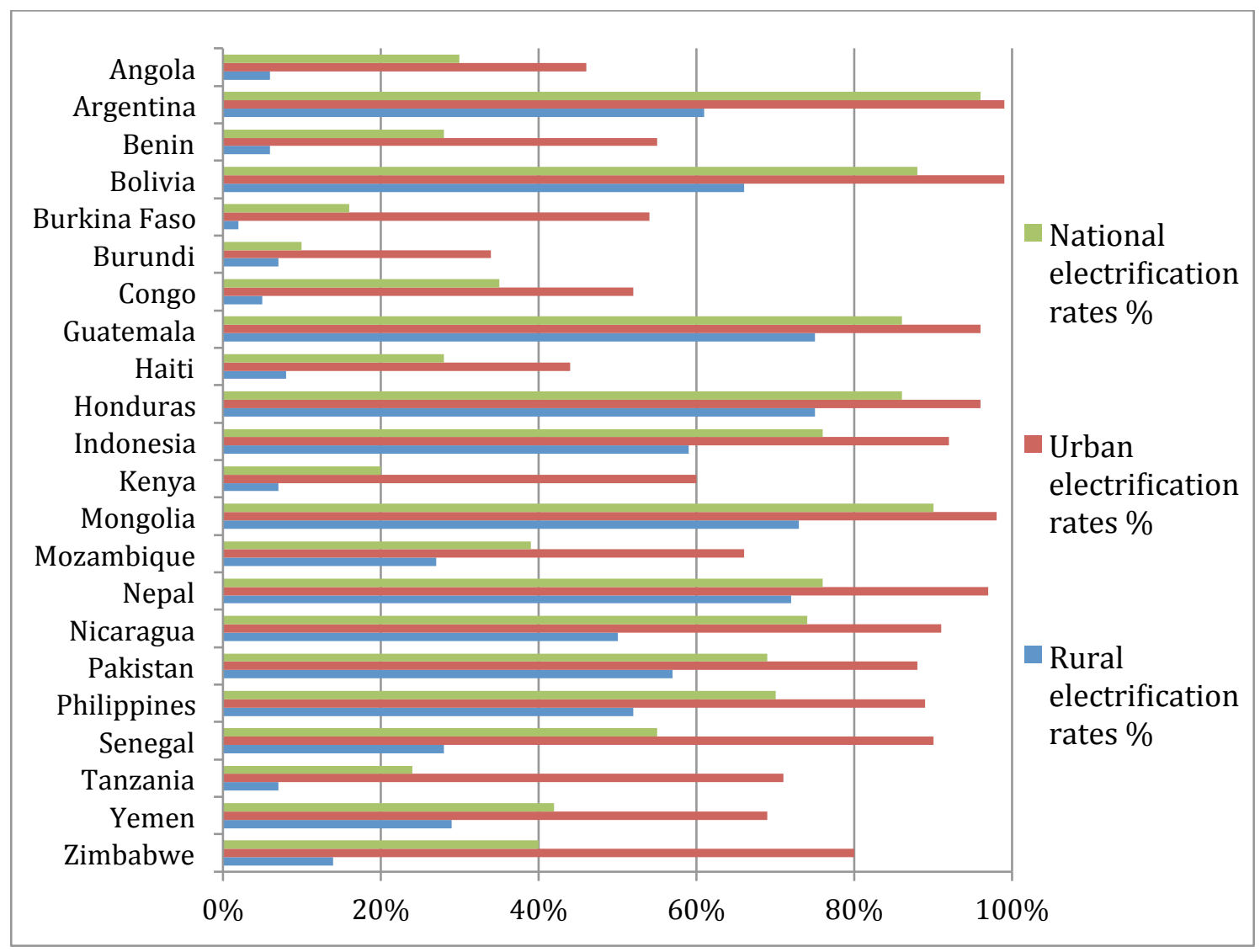

[5.8] National, urban and rural electrification rates (author's illustration, numbers from IEA, 2014) 
In Tanzania, the rural electrification rate is at 7 per cent, whereas urban electrification stands at 71 per cent. In countries like Argentina and Bolivia, the urban electrification rate is almost 100 per cent, but rural electrification rates are around 65 per cent. The rural population of countries where national electrification rates are below 50 per cent has virtually no access to electricity. The chart above demonstrates that access to electrical energy is very limited in developing countries. Many of them do not reach a national electrification rate of 50 per cent. For populations living in rural areas, electricity access is very limited, and often, less than 10 per cent of the people are able to use electricity.

\section{Conclusion:}

What do all of these numbers and figures tell us? It is obvious that low income economy countries are approximating OECD countries in energy consumption. It seems slow, but is a visible and continuous process. The figures drawn upon above also illustrate the extreme number of people living without access to electricity as well as the enormous differences in access to electricity, within continents, regions and even countries. The electrification rate in many countries seems high. Taking a closer look and comparing these numbers to the population growth and the location of where people live, one thing becomes very obvious. There is a lack of electrification located particularly in rural areas, specifically in rural areas of LCDs and Sub-Saharan Africa. 


\subsection{Rural Electrification - Outlook and Potential}

In the previous chapter, we saw that many people live without access to electricity in rural areas. Particularly in the Global South ${ }^{35}$, but also in other regions, the percentage of households lacking access to electricity is high. Many of these remote areas are very difficult to access, making it technically challenging and expensive to expand national electricity grids to include them. For that reason, it is financially more effective and practically easier to use a more decentralized energy supply approach, with local production of renewable energy. According to the figures of 2012, as mentioned previously above, 1.3 billion people, representing 18 per cent of the world's population, are living without electricity:

Since 2002, the un-electrified population has decreased worldwide by 161 million, despite the growth in world population of more than 500 million. However, if globally the situation is improving, the regional developments diverge ever more. Whereas Latin America and Asia have substantially accelerated their electrification, most of Sub Saharan Africa lacks behind and does not even keep up with population growth (Alliance for Rural Electrification (ARE]), 2010).

It is also important to ask: What are the benefits of having access to electricity? The benefits of having access to electricity are diverse. Electricity is necessary to meet some of our basic needs. Not having access to electricity means spending a lot of time, money and effort on securing energy supply for basic needs. Small amounts of energy can free up large amounts of human time and labor. Electricity also has an enormous impact in the fields of education and health. With the use of electrical light, the quality of indoor air is improved, since petroleum lighting need not be used any more. Also, refrigerators have a positive effect on

\footnotetext{
${ }^{35}$ The "Global South" refers to countries that score lower than 8 on the Human Development Index, as determined by the UN Development Program. Most of these countries are geographically located in the South. 
health. Food can be stored much longer, which potentially reduces hunger. Being able to keep vaccines and medicine cooled has a direct positive effect on health.

Replacing a diesel generator for electricity generation with a renewable energy source will make fossil fuel transportation less and less necessary. In some areas, fossil fuels are only available far away, requiring a lot of effort to reach remote areas, which also makes it more expensive. Without lighting systems, activity is limited to daylight hours. With electrical lighting, the community's daylight hours allow more time for education or all kind of productivity.

Rural electrification benefits include outcomes such as reducing poverty, creating jobs and reducing biodiversity loss. The field of rural electrification has enormous potential as it intermingles with a complex set of dynamics and interrelations. The Alliance for Rural Electrification (ARE, 2010) gives an overview on the benefits of rural electrification:

○ Education: Enhance education by allowing studying beyond daylight, introducing better learning conditions such as computer facilities, internet and distance learning if there is a lack of teachers

- Reduce Isolation: Reduce isolation and marginalization by the improvement of communication and information channels such us telephony, TV, cinema, radio and computers

- Improve Healthcare: Improve healthcare conditions by providing drinking water and lighting for rural clinics where vaccines could be conserved, blood storage refrigerators could be installed, operations could be carried out with sterilizations measures.

- Foster Productivity: Foster productivity, since electricity also allows for irrigation, crop processing, food preservation, water pumping, ice making, etc.

According to a World Bank-initiated study on the potential of "The Picohydropower ${ }^{36}$ Market for Low Income Households in Ecuador" (Energy Sector Management Assistance Program (ESMAP), 2005: 2), almost 2.5 million households are without electricity in the Andean

\footnotetext{
${ }^{36}$ Pico hydro power refers to very small hydro power units suitable for a single household or a small group of households. In this study it covers a capacity up to $5 \mathrm{~kW}$.
} 
region alone. The technically achievable number of households that could use picohydro in Bolivia, Peru, Ecuador, Colombia and Venezuela is 1,230,000 (ESMAP, 2005: 2).

\section{Remote Area Electrification through Open Source Hardware:}

Would open source hardware for rural electrification make an enormous contribution to the access gap? The following chapters evaluate the feasibility of such an endeavor, starting with the physical background of renewable energy and technical solutions for rural electrification.

\subsection{Introduction to Renewable Energy}

By definition, renewable energy is all energy that comes from natural resources (wind, sun, water, tidal and geothermal energy, for instance) and is renewable, or can be naturally replenished. The word 'energy' itself is derived from the Greek en (in) and ergon (work) (Boyle, 2004: 3). "99.9\% of the energy, which is transformed at the earth, is energy from the sun” (Kaltschmitt \& Wiese, 1997: 43). Almost all renewable energy sources are derived from the enormous power of the sun's radiation. There are two exceptions, tidal energy and geothermal energy Boyle defines tidal energy as: "the result of the interaction of the gravitational pull of the moon and, to a lesser extent, the sun, on the seas" (2014:197). The energy saved in the form of heat underneath the earth's surface is called Geothermal Energy (Hennicke \& Fischedick, 2007: 54). It is important to know, that "the net solar power input to the earth is more that 10,000 times humanity's current rate of use of fossil and nuclear fuels" (Boyle, 2004: 66).

This study focuses on renewable energy forms that are deflected from the sun. Energy from the sun is natural, endless and free. Direct solar energy is used as solar thermal energy 
(cooking, heating, water treatment, concentrated solar power, etc.) and through photovoltaic modules. This study focuses on solar power applications, which transform solar energy into electricity. Wind, hydropower and bioenergy are indirect solar energy, which can be transformed into electrical energy as well.

Renewable energy is the oldest energy source used in the history of humankind, as the following paragraph describes:

Solar Power [...] was the energy source upon which early human societies were based. [...] Societies went on to develop ways of harnessing the movement of water and wind, both caused by solar heating of the oceans and atmosphere, to grind corn, irrigate crops and propel ships. [...] Technologies for harnessing powers of the sun, firewood, water and wind continued to improve right up to the early years of the industrial revolution. But by then the advantage of coal, the first and most plentiful of the fossil fuels had become apparent. These highly concentrated energy sources soon displaced wood, wind and water in the homes, industries and transport systems of the industrial nations. Today the fossil fuel trio of coal, oil and natural gas provides three quarters of the world's energy (Boyle, 2004: 2).

Illustration 5.9 gives an overview of renewable energy sources, their harnessing, conversion, generation, storage and distribution. Renewable energy resources can be distinguished into four categories: Direct Sunlight, Biomass or Organic Waste, Water and Wind Energy. These resources have to be harnessed, with the exception of wind power and sunlight (if the energy conversion takes place through photovoltaic cells). In the case of Biomass and Water and Wind, the energy is converted into rotation, kinetic energy. This kinetic energy then is transformed into electrical energy by a generator. From there on it is distributed to the consumers or stored in battery banks. These different forms of renewable energy will be discussed in more detail in the following subchapters. 


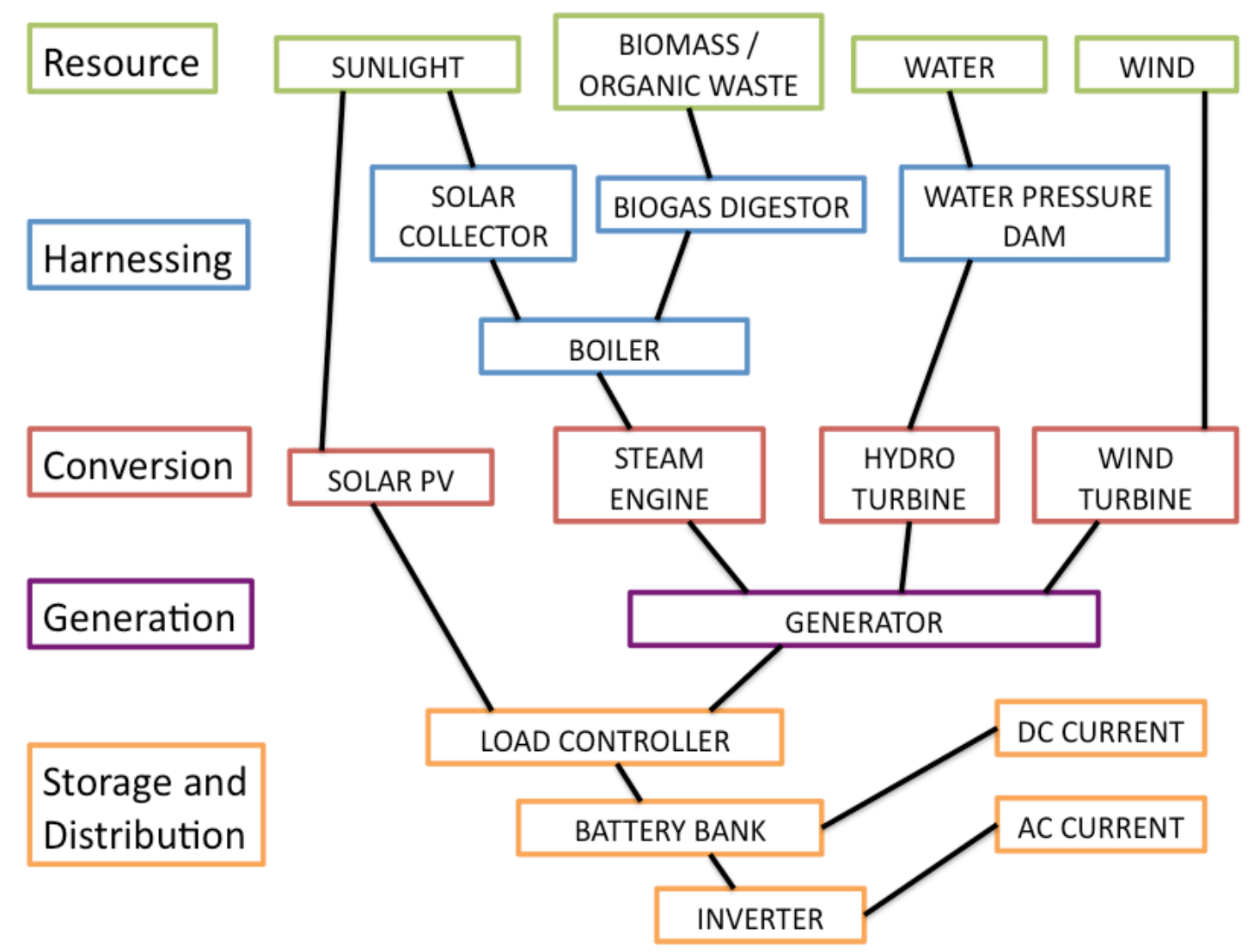

[5.9] Overview of renewable energy sources and transformation processes (author's illustration)

Small-scale renewable energy technologies avoid greenhouse emissions, "have low operation and maintenance costs, generate employment and allow decentralized production for the development of dispersed populations. Within rural areas, they are capable of electrifying homes, villages, farms and small industries, as well as being used for telecommunications, water supply and irrigation" (ARE, 2010).

We are living in a limited system known as Planet Earth. Renewable energy is crucial for this planet. The atmosphere has a limited capability for absorbing burned fossil fuels. Therefore, the world's energy demand can only be satisfied with renewable energy sources. Burning all fossil fuels available on this planet will most probably cause severe environmental problems and change the living conditions for plants, animals and humans in a drastic and unbearable 
way. The crucial limit is not the available amount of fossil fuels itself; it is the limitation of the atmosphere to absorb burned fossil fuels (Edenhofer, 2011). However, from the perspective of supply and demand, the available amount of fossil fuels is also important. The so-called "peak-oil" 37 phenomenon is already very influential on the energy price.

Apart from that, the distribution of fossil fuels is limited to a few countries, which is one significant cause of conflict and war. Renewable energies are reducing dependence on fossil fuels imports, which may help discourage conflict related to diminishing oil and gas resources. Unfortunately, like fossil fuels, renewable energy resources are also not distributed equally. The distribution of water, for example, also represents a source of conflict. Nevertheless, renewable energy is helping diversify the energy supply, therefore lessening dependence on one dominant energy resource, which might result in a more equal distribution of energy.

Another very important justification for renewable energy is the potential for local industries to create components for renewable energy plants, which "includes also service and maintenance and assists diversity, competition and democracy in corresponding countries" (Hennicke \& Fischedick, 2007: 25). The technology used for harnessing renewable energy sources could be considered "high-tech". Particularly in the last few years, this business sector grew enormously, and the role of renewable sources in the global balance of power continues to increase (IEA, 2013: 3). In other words: “The renewable energy resources [...] are at once the most ancient and the most modern forms of energy used by humanity" (Boyle, 2004: 2).

\footnotetext{
${ }^{37}$ Peak Oil is a theoretical point in time where the maximum rate of global petroleum extraction is reached. After that point, the rate of petroleum extraction will decline.
} 
Renewable energies are the most adaptable, flexible and easy-to-use technologies for isolated rural areas: "Renewable energy sources are among the least costly and most feasible solutions, since they are coming from unlimited and accessible sources, they are sustainable (minimum maintenance needs) and will cause no impact towards fragile ecosystems" (ARE, 2010). Financially, renewable energies are getting more and more competitive and the prices are decreasing (ARE, 2010). This is happening for different reasons. On one hand, increasing demand enables companies to manufacture higher quantities, which lowers prices:

The continuing maturation of the renewable energy industry in the developed world will keep on bringing down their costs. Unlike most conventional energy sources, the cost of producing energy from renewable energy sources will decrease dramatically in the future, given the necessary conditions (ARE, 2010).

On the other hand, renewable energy is in direct competition with energy solutions based on limited and decreasing natural resources. As a limited resource, the prices of crude oil and gas will inevitably increase, and renewable energy technologies will progressively become a financial alternative to fossil fuels.

Unfortunately, renewable energy sources for rural electrification are still perceived as highcost options (ARE, 2010). Yes, the installation of renewable energy systems is often very expensive compared to the capital costs of conventional energy technologies; but this comparison strongly depends on what is calculated into these costs. Often, environmental costs associated with fossil fuels are not fully accounted for. Furthermore, often operation and maintenance costs are not taken into account as the Alliance for Rural Electrification describes: "In many cases, particularly in remote locations, the low operation and maintenance costs as well as the inexistent fuel expenses and the increased reliability and the longer expected useful life of renewable energy technologies, offset initial capital costs, but 
this kind of life cycle accounting is not regularly used as a basis for comparison" (ARE, 2010). Therefore, public and private investments are mostly made elsewhere.

Bioenergy, wind, solar and hydropower are applicable for off-grid usage and also convenient for the idea of open source hardware and DIY construction kits. The following chapter depicts the physical background of transforming and using renewable energy sources in the form of electrical power and electricity.

\subsection{Technical Options for Rural Electrification}

This chapter explores existing forms of renewable energy, explains how the process of energy transformation works, and describes how these energies are used in rural electrification. This includes the physical background and a first outlook on its applicability for local production and Do-It-Yourself (DIY).

Due to their geographical location and critical mass, rural areas are mainly suitable for renewable energy off-grid applications (ARE, 2010). This research only applies to off-grid applications and systems that are directly connected to the consumer or to a battery via a charge controller. Hybrid systems are not part of this research.

\subsubsection{Wind Energy}

Wind power is the process of using the energy of moving air - what we call wind, of course which is caused due to changes of temperature, density and pressure. Gas expands when heated and contracts when cooled. Air moves from an area of low temperature to an area of 
high temperature. Warm air is lighter than cold air, and it will rise to higher altitudes. The density of warm air is less than that of cold air. Air moves from an area of high pressure and density to an area of low pressure and density. These effects, in combination with the Earth's rotation and the effect of Coriolis ${ }^{38}$, are the main roots of the Earth's wind system and are used in wind power. Boyle (2004: 245) explains this dynamic as follows: "The earth's wind systems are due to the movement of atmospheric air masses as a result of variations in the atmospheric pressure, which in turn are the result of differences in the solar heating of different parts of the earth's surface". For wind energy, the movement of air is converted through wind turbines.

Wind energy is one of the oldest forms of energy and has been used for milling grain, pumping water and other mechanical power applications for thousands of years (Steiner, 2003: 119), with attempts to generate electricity from wind dating back to the end of the nineteenth century (Boyle, 2004)

Wind power systems transform kinetic energy into mechanical rotation, or mechanical energy. Mechanical energy is the sum of potential and kinetic energy. Kinetic energy is "the energy contained in the wind" (Boyle, 2004: 248) and can be transformed into electrical energy with the help of a generator.

There are two principles of transforming the streaming energy of air into mechanical or electrical energy. One is by using the largest area possible to catch as much wind as possible (like on a sailing boat) and using it for rotation. Old windmills were mostly designed in that way, featuring huge blades with high surface area. This design works with the so-called "stagnation pressure" or "total pressure", the magnitude of which can be derived from the Bernoulli equation. With this design, it is only possible to transform a theoretical degree of

\footnotetext{
${ }^{38}$ The Coriolis force, described by the French mathematician Gustave Gaspard Coriolis in 1835, is a force that acts on objects, which are in motion relative to a rotating reference frame.
} 
12 percent of kinetic energy into other energy forms (Hennicke \& Fischedick, 2007: 48). For that reason, what we call windmills today are mostly used for pumping water. It is important to mention, that the power contained in the wind is not in practice the amount of power that can be extracted by a wind turbine. "This is because losses are incurred in the energy conversion process. In addition, some of the air is 'pushed aside' by the rotor and bypasses it without generating power" (Boyle, 2004: 249). The other principle is using aerofoil-shaped blades that use lift force of the profile. With this principle, it is theoretically possible to transform 59 percent $^{39}$ of the wind's energy into mechanical energy (Hennicke \& Fischedick, 2007: 48). Wind turbines design can vary from horizontal axis to vertical axis design, integrating 1 to 3 blades. "In general, the commonly used horizontal wind turbines have a higher efficiency than vertical ones, as well as a higher reliability due to a better rotor balance” (ARE, 2011: 18).

Usually, a wind turbine is designed to work at a specific wind speed. This is called the "design point" and generally defines the peak efficiency. At wind speeds above or below the design speed, the efficiency is the same or less, often much less: "A wind turbine generally operates at lower than its best efficiency, because wind speeds are never constant or average” (Watson, 2010).

Sketch 5.10 outlines the main components of a wind power system. The most important part is the rotor, which turns as a result of moving air masses; in other words, as the result of wind. This is where the energetic transformation from wind into mechanic (kinetic) energy happens. The rotor is connected through a shaft with the generator. For optimizing the speed of the generator and its energy transformation, a gearbox can be used in between the rotor and generator. The generator is where the second energetic transformation happens, where

\footnotetext{
${ }^{39}$ The $59 \%$ is rooted in the Betz-law, also known as theBetz-limit, discovered by the German physicist Albert Betz. It states that the maximum theoretical efficiency of wind turbines is the ratio of maximum power obtained from the wind to the total power available in the wind. This value is also called power coefficient $c_{p}$
} 
kinetic energy is transformed into electrical energy. Most small wind turbines have a permanent magnet generator and do not need a gearbox. This type of generator produces ACvoltage current, which must be rectified to DC by means of a simple bridge rectifier. Similar to PV systems, the DC-voltage can be stored in a battery bank. For in-battery charging systems, a charge controller is added to prevent the battery from over-charging (ARE, 2011).

Sketch 5.11 shows the electrical schematic drawing of a small wind turbine.
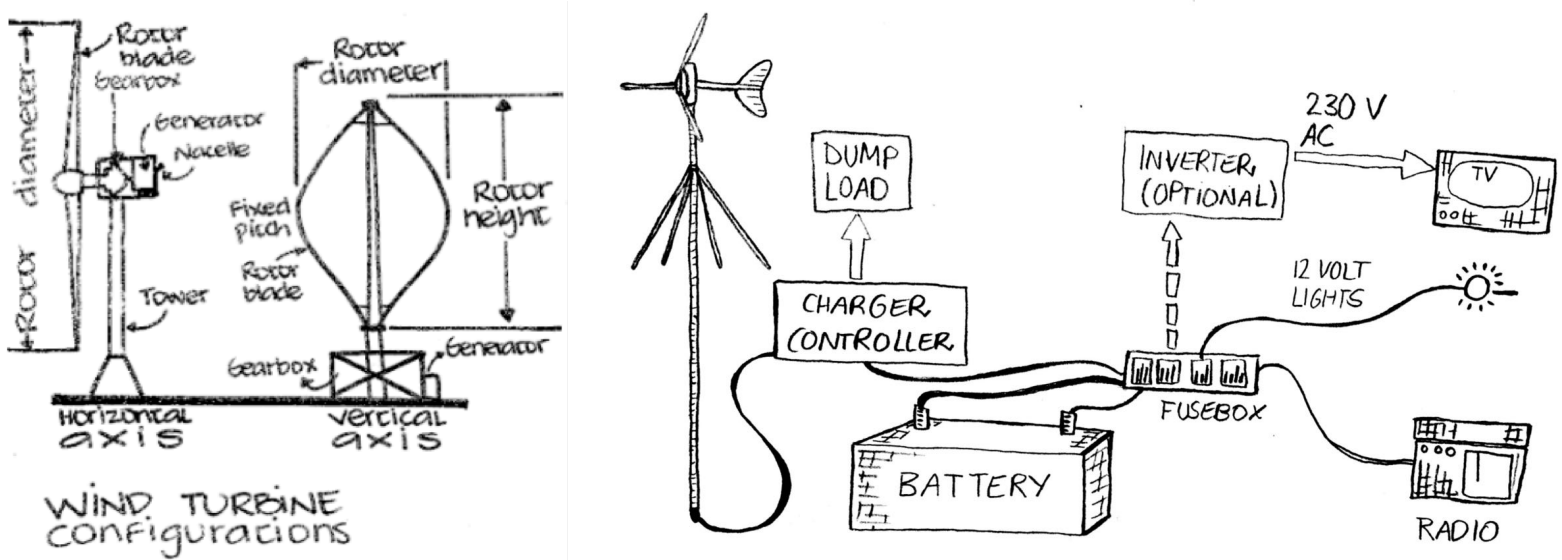

[5.10] Configuration of wind turbines, left (author's illustration, drawing by Hernandez, I.) [5.11] Electrical schematic drawing of a small wind turbine, right (author's illustration, drawing by Blatter, I.)

To a certain extent, the more blades, the higher the efficiency. Since the difference in efficiency between three or four blades is rather low, most turbines are built with three blades. A four- or five-blade design would be less cost-effective. Lowering the cost through a two-blade design, on the other hand, decreases efficiency too much. This is mainly valid for horizontal axis wind-power systems: "Nowadays, most of the machines are built horizontally with three blades and are running around 500 revolutions per minute" (Hennicke \& Fischedick, 2007: 48). Vertical wind power designs are available with all kinds of blade designs and quantities and are especially interesting for very small power outputs. 
The physical fundamental framework of fluid dynamics was discovered by the Swiss mathematician Daniel Bernoulli, who published a book called Hydrodynamica in 1738 (Encyclopedia Britannica, 2015), which considers the fundamental relationship of velocity, pressure and density of moving fluids (liquid or gas). Bernoulli's theorem states that the "mechanical energy of the flowing fluid, comprising the energy associated with fluid pressure, the gravitational potential energy of elevation and the kinetic energy of fluid motion, remains constant” (Encyclopedia Britannica, 2015). Some of these ideas are crucial for understanding the basics of wind and hydro power. The next paragraph outlines some of the basic principles of fluid mechanics.

\section{Fluid Dynamics and the Bernoulli Principle:}

Wind and hydro power turbines work with fluids (gas or liquid). The kinetic and potential energy of the fluid is transformed into mechanical energy by a turbine. The outcome is a rotation of a shaft that can be used directly or by a generator to transform it into electrical energy. Fluid mechanics is the physical field that studies fluids and the forces upon them. The following depicts only a select few of the fundamental physical laws of fluid mechanics necessary for understanding how wind or water turbines work.

Bernoulli stated that the mechanical energy of a flowing fluid remains constant. The following sketch 5.12 depicts the Bernoulli Effect. Mechanical energy consists of kinetic and potential energy. This means that the flow velocity, kinetic energy, can be transformed into pressure, potential energy, and vice versa. If the flow velocity is lower, the pressure is higher. If the pressure is low, the velocity of the flow is high. The efficiency of this transformation depends on the characteristics of the liquid in use and the way it flows. Gas has different characteristics (density, viscosity, etc.) than water while flowing. Wind and hydro power 
turbines use this effect in several ways. Sketch 5.13 depicts how the Bernoulli effect is used in aviation.

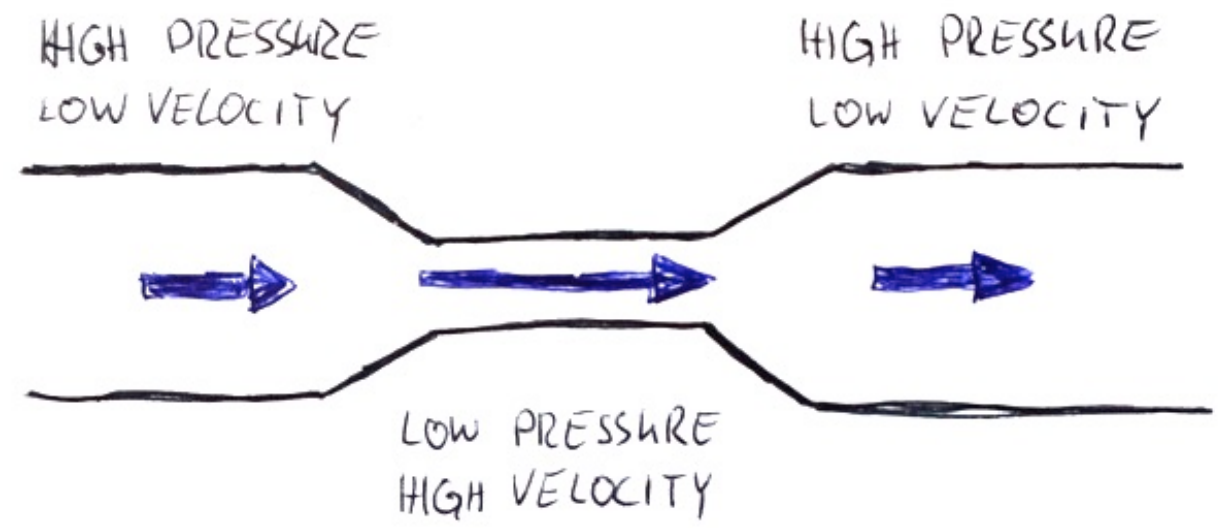

[5.12] Bernoulli Effect, (Author's illustration)

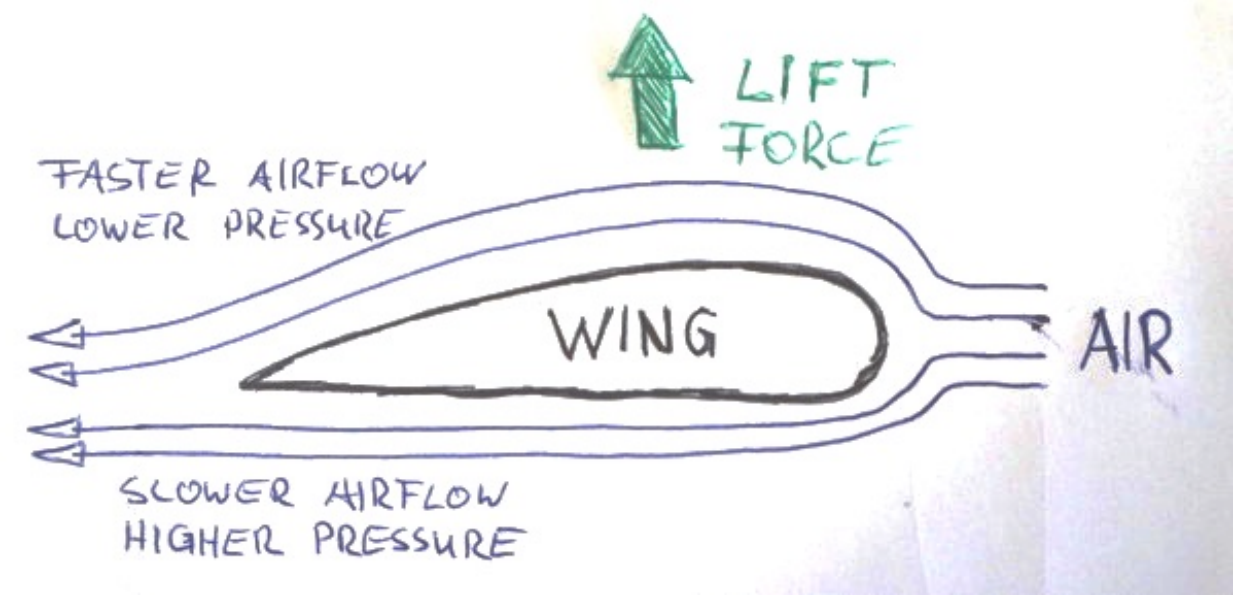

[5.13] Accelerated Airflow lowers Pressure, (Author's illustration)

Boyle describes this physical effect with the following words: "An object in an air stream experiences a force that is imparted from the air stream to that object. We can consider this force to be equivalent to two component forces acting in perpendicular directions, known as the drag force and the lift force. The magnitude of these drag and lift forces depends on the shape of the object, its orientation to the direction of the air stream, and the velocity of the air stream” (Boyle, 2004: 256). 
Due to a longer pathway on the upper side of the blade, the air moves faster. The path for the air of the lower side is shorter; therefore, the air moves slower. Since the amount of energy is constant, the difference in velocity is transferred into a pressure difference, which creates a lifting force. The drag force acts in the opposite direction of the flow. Wind power systems, which are designed with aerofoil blades (and therefore with lifting force), work with a higher efficiency than windmills.

There are two main families of windmills: vertical axis machines and horizontal axis machines. There are several technical parameters used to characterize wind turbine rotors. One of them is the power coefficient. Another is the tip speed ratio.

The power of a wind-stream is transformed into mechanical energy through slowing down the moving air mass that crosses the rotor area. For a complete extraction of power, the air mass would have to be stopped entirely. Even if the efficiency of the wind turbine is high, there is a limit. Betz's power coefficient $c_{p}$ describes the ratio of the total amount of wind energy that can be extracted theoretically if no losses occur. The $c_{p}$ theoretical maximum is at 59 percent. Practically, and especially for small wind turbines, the power coefficient is much lower. For small wind turbines, a power coefficient of around 25-45 per cent is considered a good aerodynamic solution (Noble, 2008: 2). The tip speed ratio is defined as the ratio of the speed of the extremities of a windmill rotor to the speed of the free wind. (Noble, 2008: 2).

How can we calculate the theoretical capacity of a wind power system? In order to answer this question, we have to look at the physical background and its related equations: 


$$
\begin{aligned}
& P=\frac{1}{2} \cdot \dot{m} \cdot c^{2} \\
& \dot{m}=\dot{V} \cdot \rho \\
& \rho=\frac{p}{R \cdot T} \\
& \dot{V}=A \cdot c \\
& A=\frac{d^{2} \cdot \pi}{4} \\
& P=\frac{1}{2} \cdot \dot{V} \cdot \rho \cdot c^{2}=\frac{1}{2} \cdot A \cdot \rho \cdot c^{3}=\frac{1}{8} \cdot d^{2} \cdot \pi \cdot \rho \cdot c^{3} \\
& \Rightarrow P=c_{p} \cdot \frac{1}{8} \cdot d^{2} \cdot \pi \cdot \rho \cdot c^{3}
\end{aligned}
$$

The main relationships apparent from the above equations indicate that the theoretical power depends strongly on the diameter of the rotor and the velocity of the wind:

- Diameter: The power increases with the square of the rotor diameter. Doubling the diameter increases the theoretical power output four times. For that reason, large wind power systems are in use and have increased their size in recent decades.

○ Wind Speed: The power increases with the cube of the wind velocity. Doubling the wind speed will increase the theoretical power output eight times.

In table 5.14 on the following page the characteristics of different wind turbine settings and rotor designs are depicted. We see here that the power coefficient of vertical axis wind turbines is lower than that of horizontal axis wind turbines.

Another indirect influence on horizontal axis wind turbine (HAWT) efficiency is the flow condition, rooted in the distance from the rotor axis to the ground. Higher rotor axis levels result in less turbulent wind conditions where the air flows more constantly because there are no buildings, trees, or other objects changing the wind direction and creating turbulent flow (Watter, 2011: 49). Working in less turbulent wind results in higher efficiency. Therefore, large wind turbines tend to have a higher efficiency than small wind turbines. In theory, the density of the air also has an impact, although it is not very significant or variable in practice. 
Renewable Energy and Rural Electrification

\begin{tabular}{|l|c|c|c|c|}
\hline Type & Speed & Torque & Manufacture & Power Coefficient (Cp) \\
\hline Horizontal Axis & Low & Medium & Simple & $.05-.15$ \\
\hline \hline Cretan sail & Low & High & Moderate & $.15-.30$ \\
\hline Cambered plate fan & Moderate & Low & Moderate & $.20-.35$ \\
\hline $\begin{array}{l}\text { Moderate speed } \\
\text { aero-generator }\end{array}$ & High & Very low & Precise & $.30-.45$ \\
generator & Low & Medium & Crude & .10 \\
\hline Vertical Axis & Moderate & Medium & Moderate & $.25-.35$ \\
\hline Panemone & Moderate & Very Low & Precise & $.20-.35$ \\
\hline Savonius & Moderate & Very Low & Precise & \\
\hline Darrieus & & & \\
\hline Variable Geometry & & & & \\
\hline
\end{tabular}

Table [5.14] Comparison of Rotor Types (Noble, 2008: 3)

Wind Power has great potential to be used for rural electrification. Wind power systems in small sizes are not complicated to build, and the power rate is appropriate for rural electrification. Boyle (2004: 244) writes: "Small wind machines for charging batteries have been manufactured since the 1930s". Wind energy is very cost-effective and maintenance costs are quite low.

Small wind turbines do not occupy huge parcels of land like those used for building wind farms. Because of their low energy production, they need to be located close to the source of energy consumption. The amount of energy used in transport would be too high if there were long distances involved. From both a technical and financial perspective, long transmission is not applicable for small-scale solutions. The costs for the electrical transmission would be too high. In contrast, large wind farms can prove very expensive if they are not located near the end-user or grid station. 
A precondition, of course, is a sufficient amount of wind. Weather and wind conditions can be looked up on wind maps of national weather institutions. Practically, this does not often help, since the necessary data is not always available, is not accessible or is just not helpful for small-scale communities. Microclimatic conditions have a heavy influence. If no data is available, wind measurements might be necessary prior to installation. Ideally, this type of wind mapping should be conducted over a long period of time (one year) to integrate the different seasonal variations. During site assessments, the information from local people is extremely valuable, and "what grandpa said" helps in the decision-making process.

During my research, I found several wind turbine projects of note, with different designs, both horizontal and vertical. Among others, these projects include:

○ Hugh Piggott's wind turbine

○ Chispito Wind Generator

- Zoetrope Vertical-Axis Wind Turbine

○ Vertical Axis Dynasphere Wind Module

Generally, vertical axis wind turbines (VAWT) are less efficient. On the other hand, they also have some advantages. For example, VAWT start working with lower wind speeds, do not need a specific wind direction, and are almost maintenance-free. From an aesthetic perspective, VAWT blade designs offer a wide range of creativity, which is also a crucial factor for a wind turbine. Photograph 5.15 shows the diversity of arrangements and designs. The power output range is different in these designs, but rather low (usually well below 1 $\mathrm{kW}$ ) depending on the wind velocity and the size of the wind turbine itself. Often, these designs are not completely open source. 


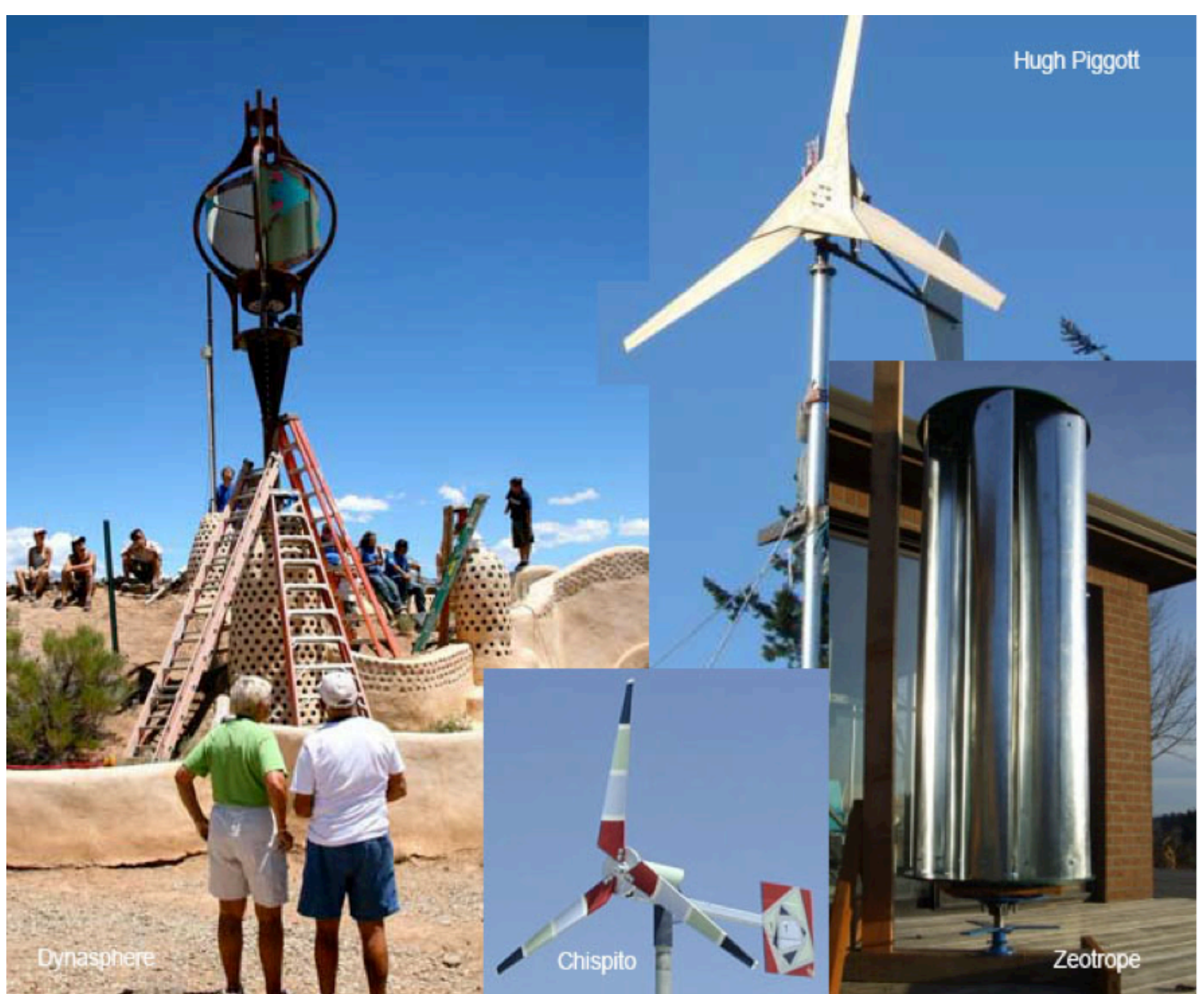

[5.15] Wind Power Design examples (author's photo collage compiled from sources clockwise from left: Earthship, Hugh Piggot, Zeotrope, Chispito. See reference list: "Zoetrope Photo Collage")

Some of the designs are accessible only in part, some are available in books, and others are only available through workshops. This creates an important networking effect. Meeting people face-to-face and getting hands-on experience will likely not be replaced entirely through new media technologies. Even in open source software like Drupal, this networking effect is used in regular "Drupal Camps" all over the world. These camps and workshops create social dynamics and foster creativity. Similarly, they bring together diverse people with diverse backgrounds working on the same problems.

Wind power systems are environmentally friendly: "The generation of electricity by wind turbines does not involve the release of carbon dioxide or pollutants that cause acid rain, 
smog or radioactivity or contaminated land, sea or water courses” (Boyle, 2004: 270). In small-scale wind turbines, noise pollution is also very low. The noise pollution of wind turbines is due to two factors. One is the mechanical noise from the equipment (generator, gearbox, etc.), and the second is the aerodynamic noise, which originates from the interaction of the airflow with the blades. As explained previously, the circumferential speed of the blades is in direct correlation with the diameter of the rotor. The circumferential speed of the rotor blades of bigger turbines is much faster as compared with that of smaller machines because of the rotor diameter. Wind farms can be noisy. The moving parts, especially the rotor, produces a constant sound, which can make life hard for a neighborhood. Small wind power systems do not reach these high speeds at the outer ends of the rotor blades. They are usually installed as single entities, and the noise pollution is therefore very low, especially with VAWT designs.

Another aspect of the environmentally friendliness of wind power systems is in regards to their interaction with birds. Wind power can be dangerous for birds, especially when groups of birds are migrating. Birds can be killed when they hit the quickly rotating propeller of a wind turbine, which occurs mostly with large wind turbines. Bird interaction with small wind turbines is much less common.

Of significant note regarding the physical background of wind turbines is that the area where wind can impact the turbine is a very important factor for how much energy can actually be transformed. Because of their design, VAWTs have a small area for catching wind, and therefore have lower potential for transforming energy. Additionally, the efficiency of this setting is lower. Nevertheless, VAWTs have significant advantages since they are easy to build, are almost maintenance free, and work at lower wind speeds. VAWTs do not depend 
on a specific wind direction. This is a great advantage, especially for the open source approach and DIY construction kits. Despite the fact that the efficiency is rather low, VAWTs seem appropriate to use as DIY decentralized energy solutions and are quite promising in their potential.

\subsubsection{Biomass Energy}

In bioenergy the sun's energy is saved in organic matter. The term bioenergy describes all energy that can be derived from materials of biological resources. "Biomass" can be "produced in forestry (woody biomass), in agriculture (energy crops) or from part of waste derived from different kinds of (biomass residues.) Biomass is the term used to describe all plant-derived material and biomass resources can be found in almost any part of the world" (WISIONS, 2010: 20). There are several technological ways to transform bioenergy into electricity. There are at least four main steps::

1. Biomass supply / collecting biomass (agriculture, forestry, organic waste, etc.)

2. Conditioning biomass (preparing the material for energy conversion, includes size reduction, cleaning, drying, etc.)

3. Energy conversion (e.g. through an energy-generator set or a fuel cell)

4. Transport and Storage (may be necessary between the outlined stages above)

Biomass can be transformed into electrical power via different transformation techniques. The following figure depicts schematically the four main transformation paths for generating electrical power out of biomass. Direct combustion, gasification and vegetable oil production are not adequate for household electrification. The necessary amount of biomass and organic waste needed for electrification is not available in such quantities for small families. Additionally, the technical complexity and financial expenditure for the energy conversion required are quite high for families to consider. 


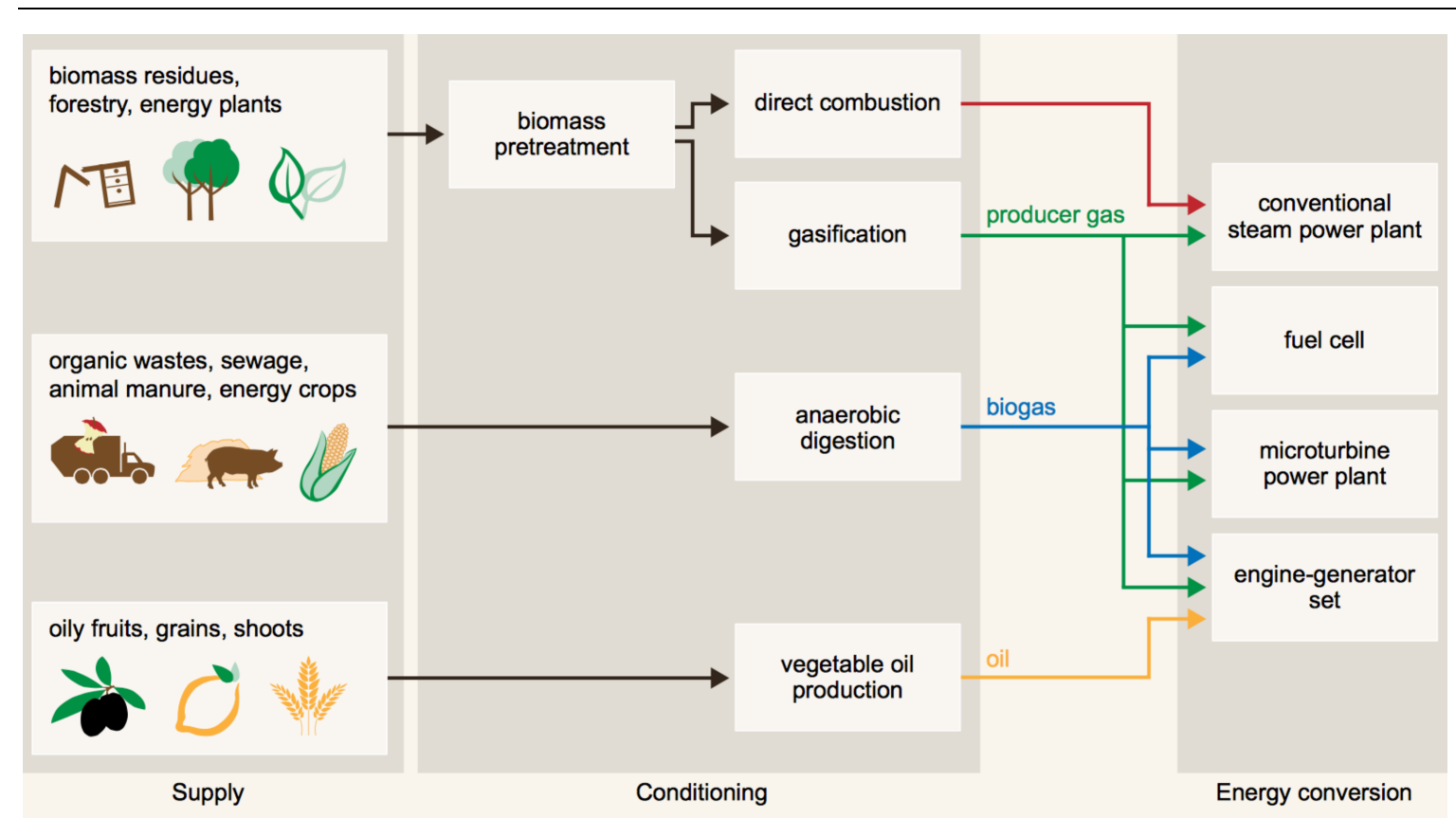

[5.16] Schematic view of some transformation paths to generate electric power from biomass (WISIONS, 2010: 20)

For rural electrification, biomass conditioning as biogas seems appropriate. Biogas is a wellestablished fuel for cooking and lighting in a number of countries. The biogas process is known as anaerobic (without air) digestion, and provides a clean cooking and lighting fuel. In other words: "Biogas is a product of breaking down organic matter in the absence of oxygen” (WISIONS, 2012: 22). Biogas can be produced on a scale varying from a small household system to a large commercial plant of several thousand cubic meters, which is a more deployed use-case.

As the schematic view 5.17 on the following page depicts, biogas could then be used to power a fuel cell, a turbine or an engine-generator set. For electricity generation, fuelconventional engine-generator sets are principally used. The combustion engine creates the rotary motion for the shaft, which is transformed through a generator into electrical power. Knowledge of the fundamental processes involved in methane fermentation is necessary for planning, building and operating biogas plants. Furthermore sufficient "fuel" must be 
available over the year "or supplies will be inconsistent and people will lose confidence in the technology" (Bates, 2007: 2).

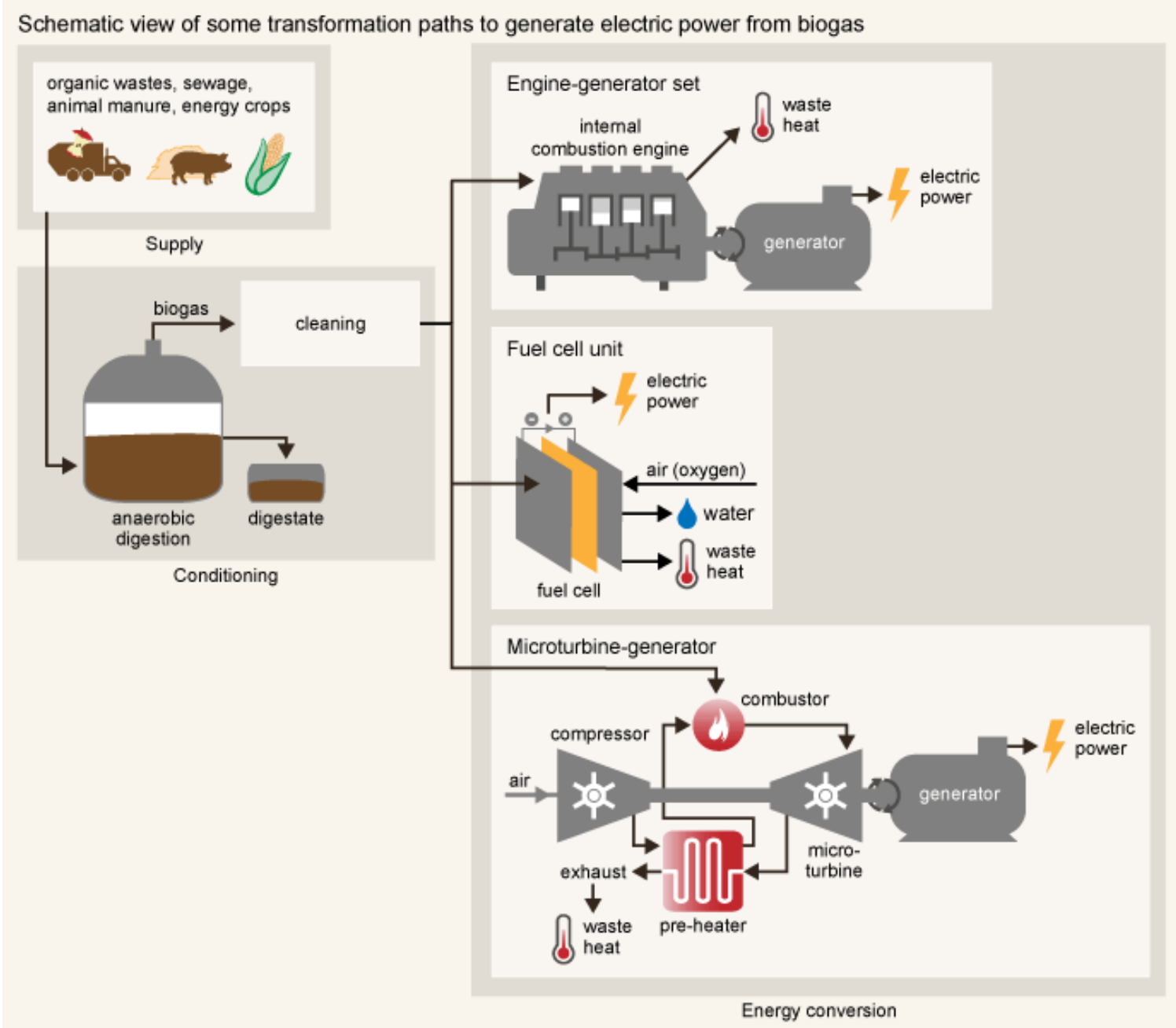

[5.17] Schematic view of some transformation paths to generate electric power from biogas (WISIONS, 2010: 22)

Another important detail is the adequate design and construction of the plant. For example, the interior of the plant has to be temperature- and pressure-resistant, which requires adequate skills and know-how. Furthermore skills are needed also "to build and to maintain biogas plants. Many units built in the past have been abandoned for lack of servicing skills" (Bates, 2007: 2).

In the process of this research, I was in contact with the local group of Engineers without Borders (EWB) in Berlin, a non-profit organization that plans and implements technical 
projects in the field of sustainable technical development work. EWB is a decentralized organization with engineers all over the world. One of its strengths is that project work is based on local working groups, while on the national and international levels people work on specific topics, like renewable energy, intercultural communication, etc. Technology is developed in a network, and the project itself is accomplished locally; thus, the technical solution for biogas, as well as the organizational approach of EWB, is close to the OS approach.

After successful accomplishment of a feasibility study in 2008 under the administration of the local EWB group in Berlin, a biogas plant concept was developed and tested at two universities in Germany. This also included an artificial greenhouse to simulate tropical climate conditions and grow "fuel" for the biogas plant. In cooperation with the Tanzanian NGO MAVUNO ${ }^{40}$, the biogas plant was implemented in Tanzania (BiogasT, 2012). This technically successful initiative won the German Innovation Prize for Climate and Environment $2011 .^{41}$

The goal of this project was to develop and build decentralized small-scale biogas plants for energy use in cooking and other fields. The fifth phase of this project started in November 2011 and primarily includes capacity building in Tanzania, signaling that the implementation, evaluation and optimization phases have been successfully completed. With the success of this project in mind, and due to the scale of this study, I focus here on wind, hydro and solar power.

\footnotetext{
${ }^{40}$ MAVUNO is a non-governmental project based at Ihanda in Karagwe District, Kagera Region in North-West Tanzania, founded 1993 by 34 rural farmer groups there. MAVUNO's name (the Swahili word for harvest) stands for its grassroots-oriented approach to improve income and living standards in rural communities of Karagwe (MAVUNO, 2013)

${ }^{41}$ Gewinner des deutschen Innovationspreises für Klima und Umwelt 2011
} 


\subsubsection{Solar Energy}

As explored previously in the introduction chapter on renewable energy, the net solar power input for the Earth is enormous. A square meter on the Earth's surface easily receives hundreds of watts, depending on the degree of latitude. ${ }^{42}$ Harvesting the sun's radiation on earth would undoubtedly contribute to reducing energy scarcity, if not solve the energy problem on Planet Earth. The following pages describe two methods of directly transforming the sun's energy into electricity: photovoltaic and concentrated solar power.

\section{Photovoltaic:}

In photovoltaic power, solar energy is converted into electricity in a solid-state device: "The term "photovoltaic" is derived by combining the Greek word for light, photos, with volt, the name of the unit of electromotive force - the force that causes the motion of electrons. The volt was named after the Italian physicist Count Alessandro Volta, the inventor of the battery. Photovoltaic thus describes the generation of electricity from light (Boyle, 2004: 66).

A photovoltaic cell, or solar cell, uses the characteristics of silicon, a special type of melted sand. These modules are made from a number of materials and fabricated in a variety of different designs. The cell consists of two or more thin layers of semi-conducting material with opposite charges - one positive, one negative. When sunlight shines on these materials, it frees electrons from fixed sites. The wavelength of the sunlight absorbed depends on the "band gap" of the material. The materials are designed so that the electrons cannot return to these sites easily, except by flowing through an external circuit, thus generating a current (Ahmed, 1994: 49). In brief, a photovoltaic cell generates electricity when photons (light) emitted by the sun strike the cell. The photovoltaic effect was discovered by the French

\footnotetext{
42 "The average energy flux at this distance on a surface perpendicular to the sun's rays is about $1353\left[\mathrm{~W} / \mathrm{m}^{2}\right]$ (Tipler, 1987: 316)
} 
physicist Edmond Becquerel. In an 1839 published paper he described his experiments "with a 'wet cell' battery, in the course of which he found that the battery voltage increased when its silver plates were exposed to light" (Boyle, 2004: 67). The working principle of a solar cell is very well explained on the Strathclyde University website, as follows:

When the sunlight strikes the solar cell, electrons are knocked loose and move toward the treated front surface of the solar cell. This creates an electron imbalance between the front and back of the cell and causes electricity flow - the greater the intensity of light, the greater the flow of electricity (Strathclyde University, n.d.).

The PV cell has no moving parts and could theoretically operate for an indefinite period of time without losing its efficiency. In practice, this is not yet possible. In normal operation, photovoltaic energy systems do not emit any gaseous or liquid pollutants or radioactive substances. Boyle writes, however, that " small amounts of toxic chemicals are used in the manufacture of some PV modules" (2004: 95-96). In addition, photovoltaic technology is a clean renewable energy source, and PV modules have no moving parts, making them safe in the mechanical sense and implying that there is no noise emission and very few maintenance requirements.

A stand-alone PV system can be defined as an off-grid system with one or several solar PV modules. According to their size (power output), Different size models (based on power output) exist on the market and are grouped as follows::

○ PicoPV Systems (PPS)

○ Classical Solar Home Systems (SHS)

○ Solar Residential System (SRS) (ARE, 2011: 9)

Due to its limited power output, PPS systems are mainly used for lighting and mobile phone charging. The advantage is its small size and low price. However, the power potential is very low, so this study does not include PPS. 
The SHS, perhaps the most well-known and installed type of renewable energy system around the world, is already nearly thirty years old and is the symbol of PV standalone systems. The application of SHS includes lighting and phone charging, as well as radio and television. According to the Alliance for Rural Electrification the prices range from "€140 to $€ 1.600$, whereas many SHS remain within payment capacity of rural end-users and are commonly sold without any subsidy" (ARE, 2011: 10). Figure 5.18 shows a schematic view for an SHS application composed of several independent components: PV modules, charge controller, battery and the consumers. The central component of the system is the charge controller, who is responsible for the overall energy management (ARE, 2011: 10). Figure 5.18 also depicts the simplicity of the technology.
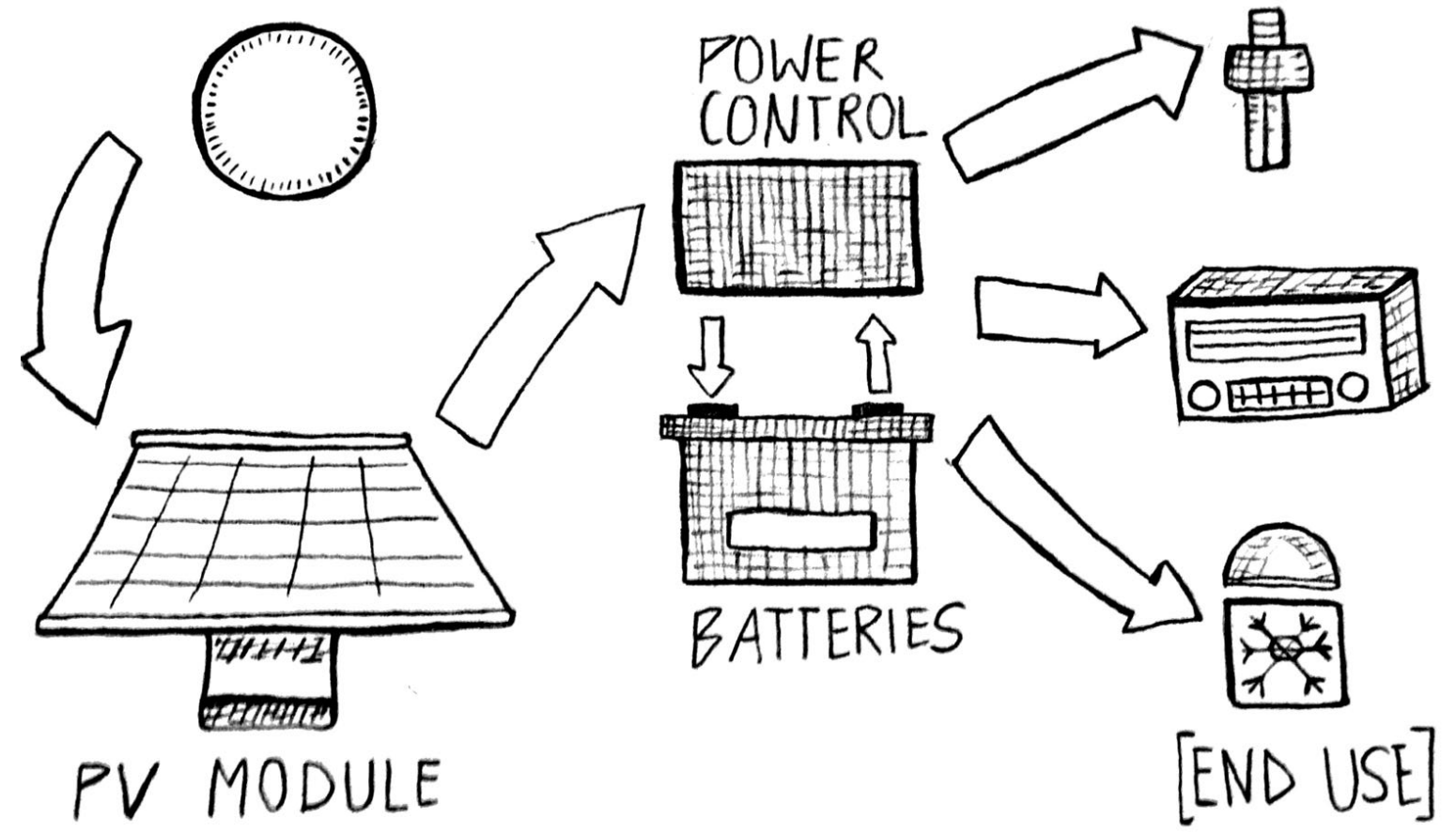

[5.18] Schematic view of an off-grid PV-System (author's illustration, drawing by Blatter, I.)

SRS start with a potential capacity of about 500 [W] up to several $[\mathrm{kW}]$ and therefore offer a wide range of applications. SRS often include an inverter allowing the use of AC loads (ARE, 2011: 9-10). Because of the size and higher investment costs, this study is limited to 
"classical" SHS systems, which are able to provide a decent amount of energy for a family. In off-grid areas far away from the grid, with no access to water or wind power, photovoltaic energy can be highly competitive with other forms of energy supply - especially in countries with a high solar radiation level. In addition, "rapid progress in increasing the efficiency and reducing the cost of PV cells has been made over the past few decades" (Boyle, 2004: 69).

The only environmental impact of PV systems is an aesthetic one, since rooftop panels will be visible to neighbors, as already discussed in the wind power section. Unfortunately, PV cells are still rather expensive in their production and therefore not very cost effective. As of today, there is no other way to manufacture solar panels, and even if the supply of panels produced has increased in the last few years, which lowers the price, it is still too high compared to other energy sources. Apart from the production of cells, solar photovoltaic energy seems to be an applicable technical solution for off-grid rural electrification.

Unfortunately, the technology of PV seems to be limited in its applicability as an open source hardware approach. A PV cell cannot be made without proper equipment, tools and material resources. As such, it is very difficult to become independent from a PV cell manufacturer. Perhaps this will change through technical development in the near future.

\section{Solar Thermal Energy:}

Solar thermal technologies harness the sun's radiation and transform it into heat. Solar thermal technology is principally applied for crop drying, water heating, cooking and distillation. Solar thermal energy is among the oldest forms of energy transformation, as Punter states: "Many solar thermal technologies have existed for centuries and are wellunderstood. They have established manufacturing bases in many sun-rich countries. Unlike 
photovoltaic technologies, manufacturing can be done on a small scale without using expensive equipment" (Punter, 2011: 1). Concentrated solar thermal power systems (CSP) use mirrors and tracking systems to focus a large area of sunlight into a small beam. More advanced and sophisticated designs can be also used to generate electricity. Kulsum Ahmed $(1994,31)$ explains that the methods "of solar-thermal power generation are essentially the same as conventional technologies, except that the 'fuel' is direct heat energy rather than stored energy in the form of fossil fuels, from which the heat energy needs to be released by combustion" (Ahmed, 1994: 31).

Today, there is a wide range of concentrated solar thermal power technologies available on the market. For cooking, CSP systems mainly use parabolic mirror systems to concentrate a large area of sunlight into a small area. The following sketch depicts the three main types of systems in use for concentrating and collecting diffuse sunlight. The principle behind these forms of collection is the same: The sun's radiation is harnessed on a large area and then, via mirrors, focused to one point.

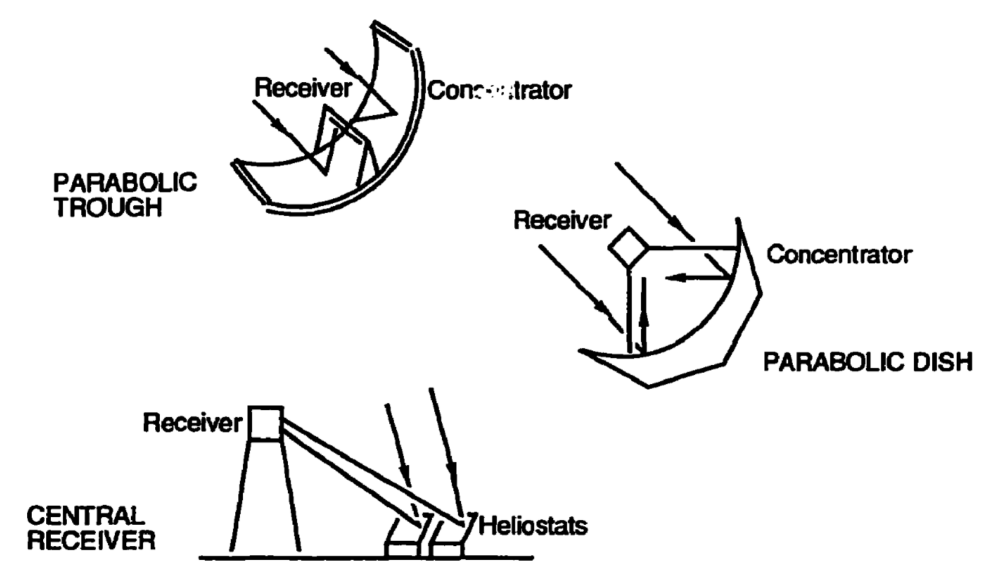

[5.19] Concentrator and Receiver Systems for Solar Thermal Electric Technologies (Ahmed, 1994: 34)

In the case of generating electricity, the sun's rays are used to heat a fluid. The heated fluid, in form of steam, can then be used to drive a turbo generator" (Ahmed, 1994: 31). Because 
of this physical multistep process, the generation of electricity from solar thermal heat is technically extensive.

In my research, I found an open source concentrated solar power project called Solar Fire ${ }^{43}$. The mission of GoSol, under whose auspices Solar Fire operates as an open source initiative, is stated on its website as follows: "Through its eco-system, GoSol's mission is to eradicate energy poverty and minimize the damage of climate change by spreading DIY tech and breaking down the barriers to solar energy access" (GoSol, 2015). GoSol's main product is a lightweight, commercial-class solar concentrator design to be built locally to lower cost and increase autonomy (GoSol, 2015).

The following photographs depict a Sol4 model built in Finland and its replication in Islamabad, Pakistan.
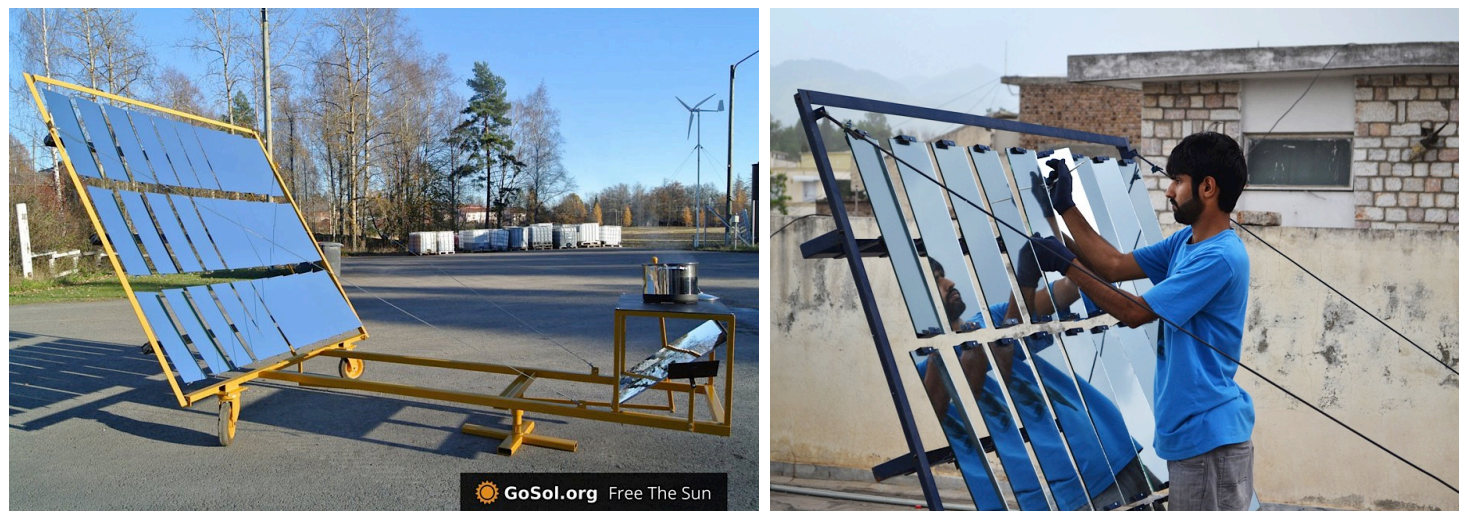

[5.20] GoSol solar concentrator and its replication in Islamabad, Pakistan (GoSol, 2015)

GoSol is about to release construction guides to build a solar concentrator. Early adopters are already able to access these manuals, and their release for the wider public is scheduled for fall 2015.

The uses for this solar concentrator include roasting, dehydrating, baking and cooking. In India, it was once successfully tested to run a steam engine. However, this test was only run

\footnotetext{
${ }^{43}$ Over the course of my research project, solarfire.org transformed into a "social" company based in Tampere, Finland. Today, the open source branch exists as an "initiative" under the name gosol.org (Source: personal conversation with Urs Riggenbach in Bern 26.07.2015)
} 
for a very short time, and this technology is still far from being a promotable configuration and design. Results have shown that none of the installments produce electricity. The capability to generate electricity out of small-scale concentrated power systems is still very difficult, as Urs Riggenbach explains: "You can use concentrated solar power for all applications connected to heat. For electronic electricity demand, you have PV panels" (personal conversation, July 26, 2015).

GoSol has proven to provide an important contribution to open source renewable energy in the form of solar concentrated power. However, its application range is limited to cooking, baking and other thermal applications.

\subsubsection{Hydropower}

Last but not least, there is the technical option of transforming the potential and kinetic energy of water into electrical energy. Figure 5.21 explains the world's water cycle, which determines water availability on Planet Earth. The origin of this cycle is, again, the sun's energy, which powers this remarkable process.

The sun's energy in the form of heat and light causes water to evaporate from oceans, lakes and rivers. The vaporized water then rises to the atmosphere, lifted up by warm air currents from the Earth's surface, and stored in the atmosphere. "When the air currents reach the cooler layers of the atmosphere, the water vapor condenses around and clings on to fine particles in the air. This step is called condensation" (Foundation for Water and Energy Education, 2015). As the air gets increasingly moist and humid, these particles begin to form clouds, and inevitably, the water will fall down to the Earth's surface again in the form of rain or snow. This process is called precipitation. Much of the water will go into streams and 
rivers at different altitudes. The Earth's surface is not flat, and therefore, water has a potential energy. Ultimately, it will flow to lower altitudes, and eventually into the ocean at sea level. From there, the cycle starts again.

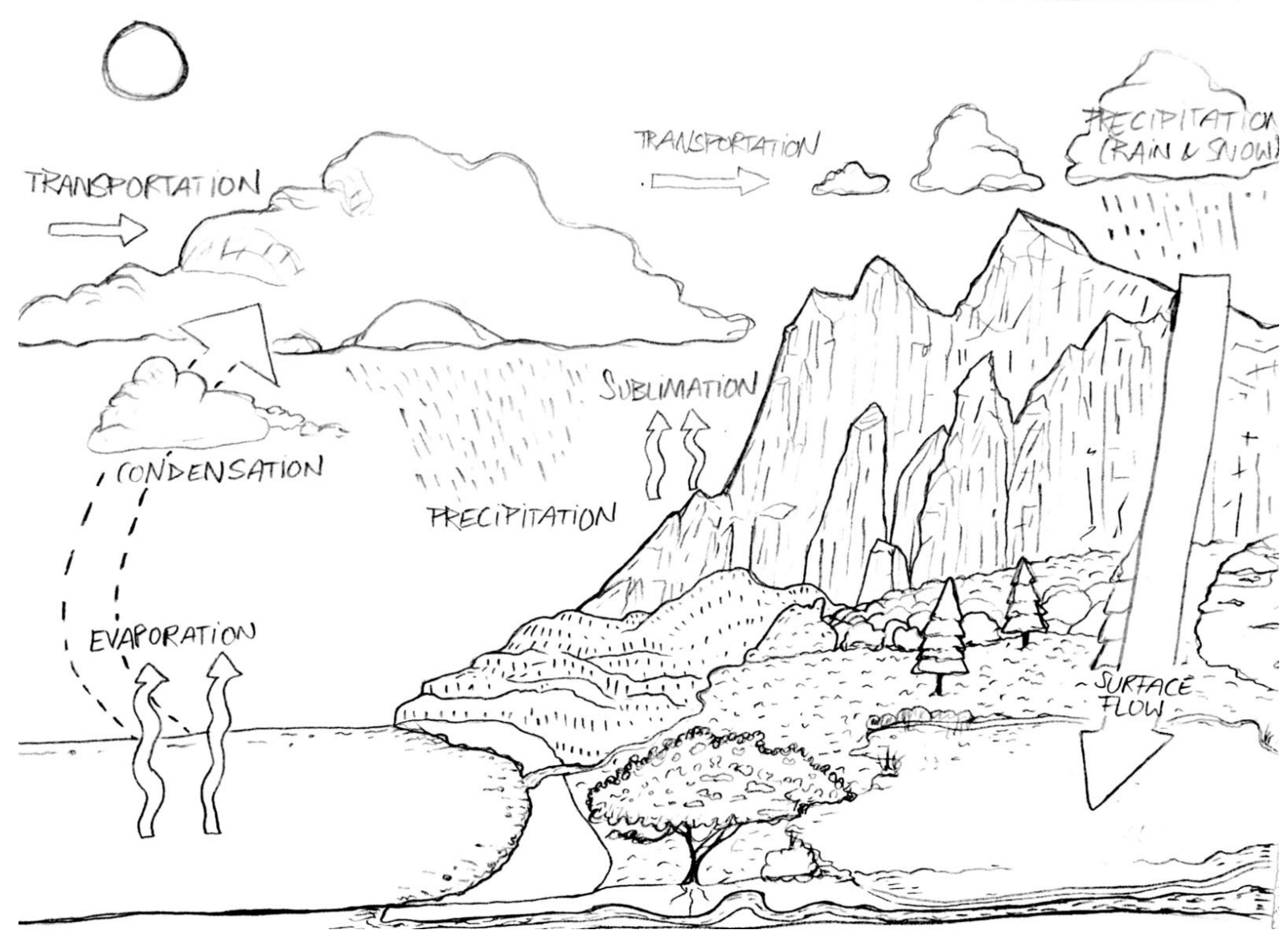

[5.21] The Hydrologic Cycle (author's illustration, drawing by Blatter, I.)

The principle behind generating electricity from water is quite similar to wind power; however, the medium of water has a much higher density and is caught vertically rather than horizontally ${ }^{44}$ as it is in wind turbines. Another important difference is that in hydropower the medium is directed towards a turbine, whereas in wind power it is a free flow of air. A hydroturbine converts energy in the form of falling water into rotating shaft power. A hydroelectric plant consists of the following components:

${ }^{44}$ With the exception of bulb turbines, which are a special form of Kaplan turbines oriented on a horizontal axis. 
○ Civil Works (damn, Intake, Weir, etc.)

○ Penstock

○ Powerhouse and Electromechanical Equipment (Turbine, Gearbox, Controller, Generator)

- Electrical Transmission and Distribution

The following chapter provides the physical background of hydropower harnessing and conversion principles.

Physical Background of Hydro Power:

Hydro-energy is available in two forms: in potential energy from high heads of water retained in dams, and kinetic energy from current flow in rivers. ${ }^{45}$ Like wind power, hydropower is a very old method of transform energy and has been done in many different ways, as Woodbank states: "Many ingenious ways have been developed for harnessing this energy but most involve directing the water flow through a turbine to generate electricity" (Woodbank, 2005). As a wind turbine does with wind, a hydro-turbine converts the kinetic energy of water into rotational motion of the turbine shaft. The working fluid (water) then flows back into the river (tailrace), ideally with almost no energy left.

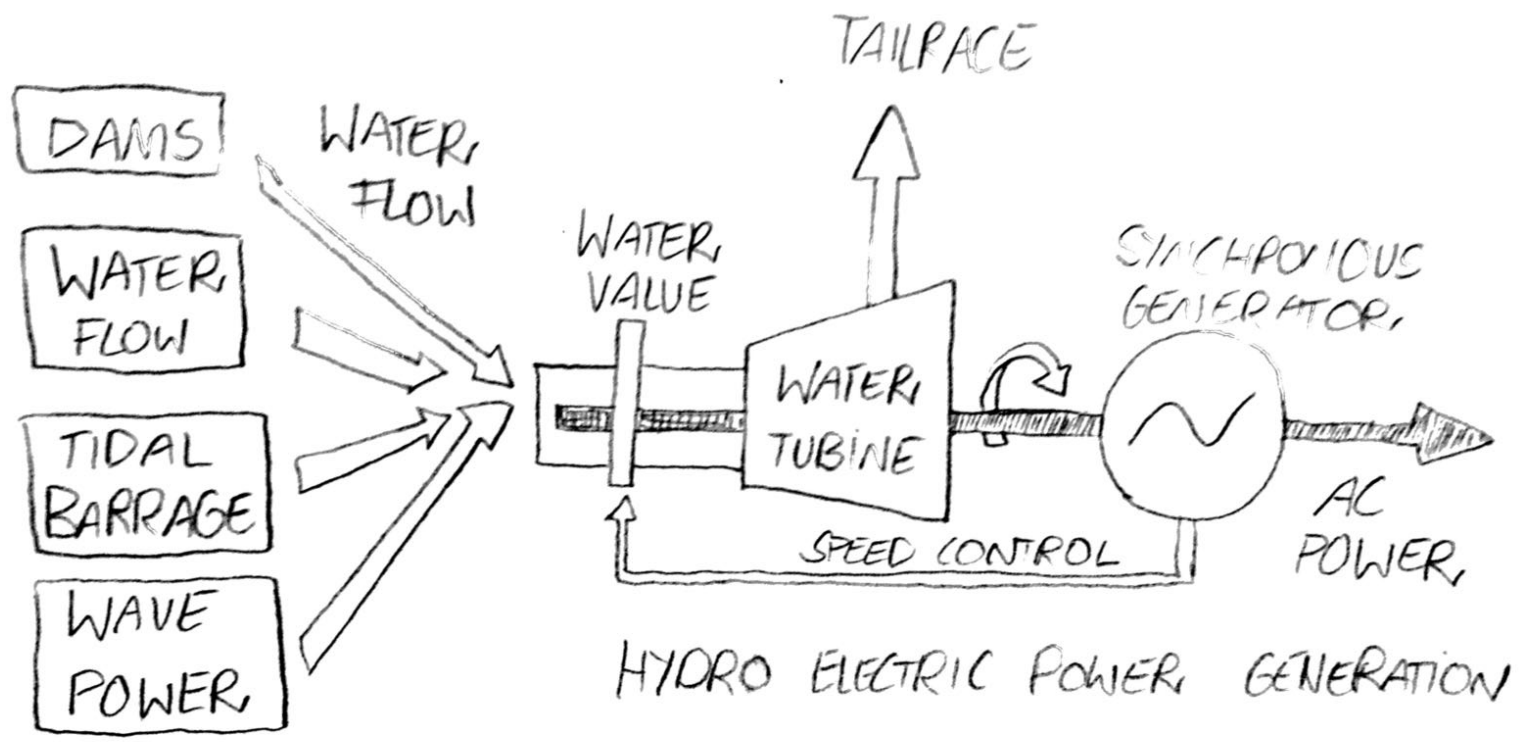

[5.22] Hydro-electric power generation, (author's illustration, drawing by Blatter, I.)

${ }^{45}$ Kinetic energy from tidal barrages or from the movement of waves is not part of this study. 
The turbine shaft is connected to a generator, if necessary through a gearbox. The generator transforms the rotation of the shaft into electrical energy in the form of alternating power (AC). Schema 5.22 depicts the hydroelectric power transformation process as described.

Due to the different application conditions in height and flow, different types of turbines have been developed to optimize the performance and efficiency of energy transformation, as Woodbank (2005) describes: "Water turbines depend on the impulse of the working fluid on the turbine blades or the reaction between the working fluid and the blades to turn the turbine shaft, which in turn drives the generator"

The fundamental relationship of pumps and turbines was discovered by Swiss mathematician Leonhard Euler in 1754. Euler demonstrated that "the torque on the shaft is equal to the change in angular momentum of the water flow as it is deflected by the turbine blades and the power generated is equal to the torque on the shaft multiplied by the rotational speed of the shaft" (Woodbank, 2005). The only thing that matters is the change in angular momentum of the fluid between the turbine's (or pump's) input and output. This is valid for all kinds of turbines. With the help of these equations, the geometry of the head of the turbine (or pump) can be determined. The illustration 5.23 on the next page explains Euler's Turbine Equation with velocity triangles of turbine input and output.

A seminal work on hydroelectricity was written by the German-Hungarian civil engineer Emil Mosonyi. Among hundreds of other publications, he published Water Power Development in the first half of the 20th Century. This two-volume book covers a wide range of aspects on hydroelectric power usage, describes and gives examples for hydroelectric designs and settings, and also formulates the basic equations and physical background for the 
use of this technology. It also states that the essential characteristics of a hydro site are a result of two factors: the height (the height $(\mathrm{H})$ through which water falls in meters) and the

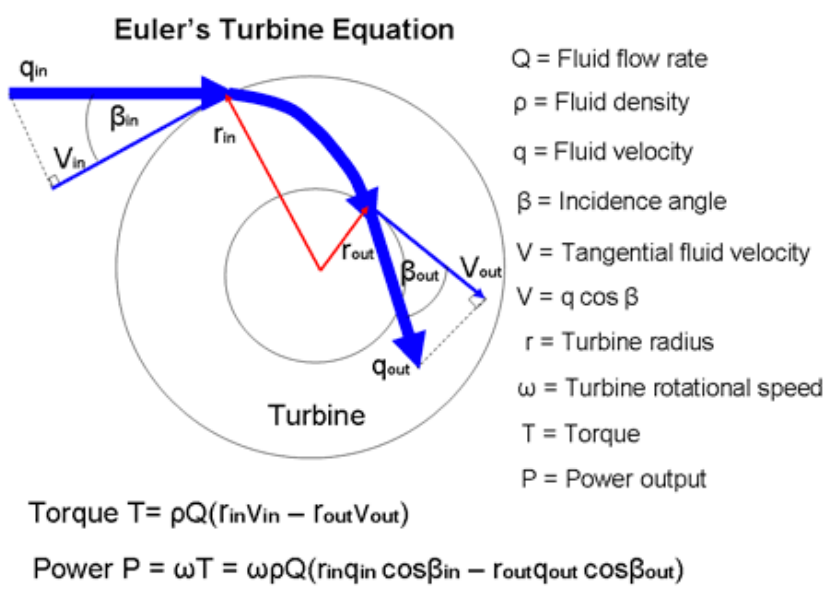

[5.23] Euler's Turbine Equation (Hydroelectric Power, n.d.)

flow (the flow (Q) in cubic meters per second) ${ }^{46}$ of water. According to Mosonyi (1987: 151), a basic equation can be used to estimate the potential of transformable energy carried out by water:

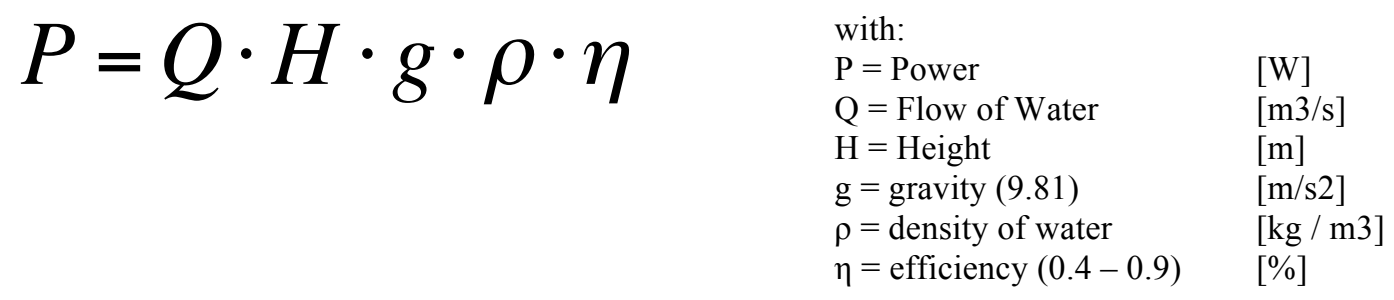

In hydropower, the maximum possible energy extractable from water is defined through the product of water flow, height difference (head), gravity, density and a factor of efficiency. The only variables in this equation are flow and height, whereas it is always desirable to maximize efficiency.

Unfortunately, "in any real system the water will lose some energy due to friction drag and turbulence, and the effective net head will be less than the gross head" (Boyle, 2004: 156).

\footnotetext{
${ }^{46}$ In the case of rural electrification, the unit used is liters per second $[1 / \mathrm{s}]$ instead of cubic meters per second $\left[\mathrm{m}^{3} / \mathrm{s}\right]$ 
Power loss is valid for all parts of a hydroelectric scheme. The following sketch 5.24 depicts typical losses for each part of the installation.

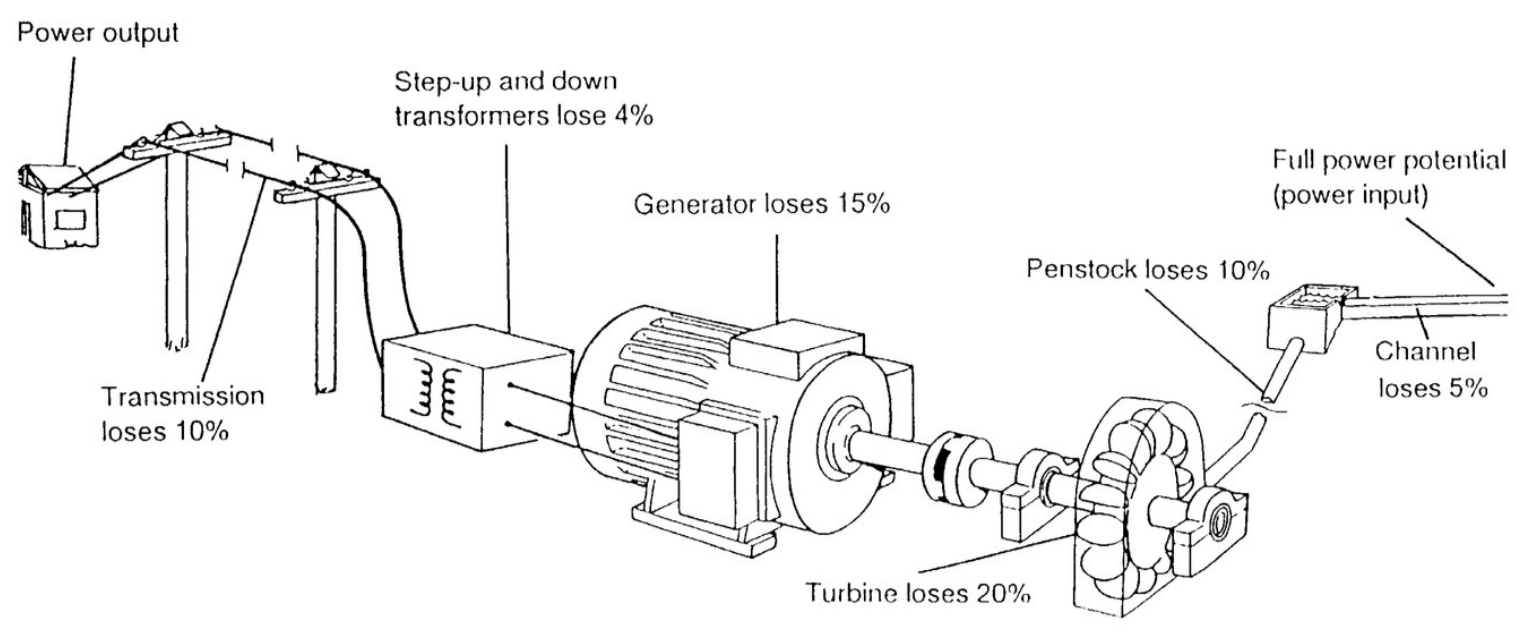

[5.24] Typical System Efficiency for a Scheme Running at full Design Flow (Harvey, 1993: $4)$.

The conversion of hydraulic power to mechanical power by means of a turbine is connected to losses. Part of the power is lost in the form of heat and noise. The efficiency quantifies these losses. and the overall efficiency of the system is the product of all losses as follows:

$$
\begin{aligned}
\text { Overall efficiency } & =e_{\text {civil works }} \times e_{\text {penstock }} \times e_{\text {turbine }} \times e_{\text {generator }} \times e_{\text {power input }} \\
& =0.95 \times 0.9 \times 0.8 \times 0.85 \times 0.96 \times 0.9 \times \text { power input } \\
& =0.5[-]
\end{aligned}
$$

These values are an example for pico hydropower, where the overall efficiency of the system is around 35 to 60 per cent. In large hydropower, the factors of gravity [9.81] multiplied by efficiency $[0.9 \mathrm{x}]$ is equal to approximately 90 per cent or above. The losses through the water flow vary greatly from system to system and depend strongly on the turbine setting: "In some cases the effective head is not more than 75 per cent of the actual height difference, in others as much as 95 per cent" (Boyle, 2004: 156). Additionally, there are other energy losses due to friction, among other causes, in the plant itself. A hydroelectric power plant running in optimum conditions can be one of the most "efficient machines, converting all but a few 
percent of the input power into electrical output" (Boyle, 2004: 156). Nevertheless, the efficiency - the ratio of output to input power - is always less than $100 \%$.

The following chart [Figure 5.25] depicts energy efficiency for different energy conversion technologies. As we can see, large hydropower is by far the most efficient energy transformation technology, followed by large gas-fired CCGT (Combined Cycle Gas Turbine) power plants. ${ }^{47}$ The efficiency of CSP, biogas and wind turbines is much smaller if they are applied in the range of a few kilowatts. From the perspective of efficiency of energy transformation, large hydropower is the only technology that works efficiently.

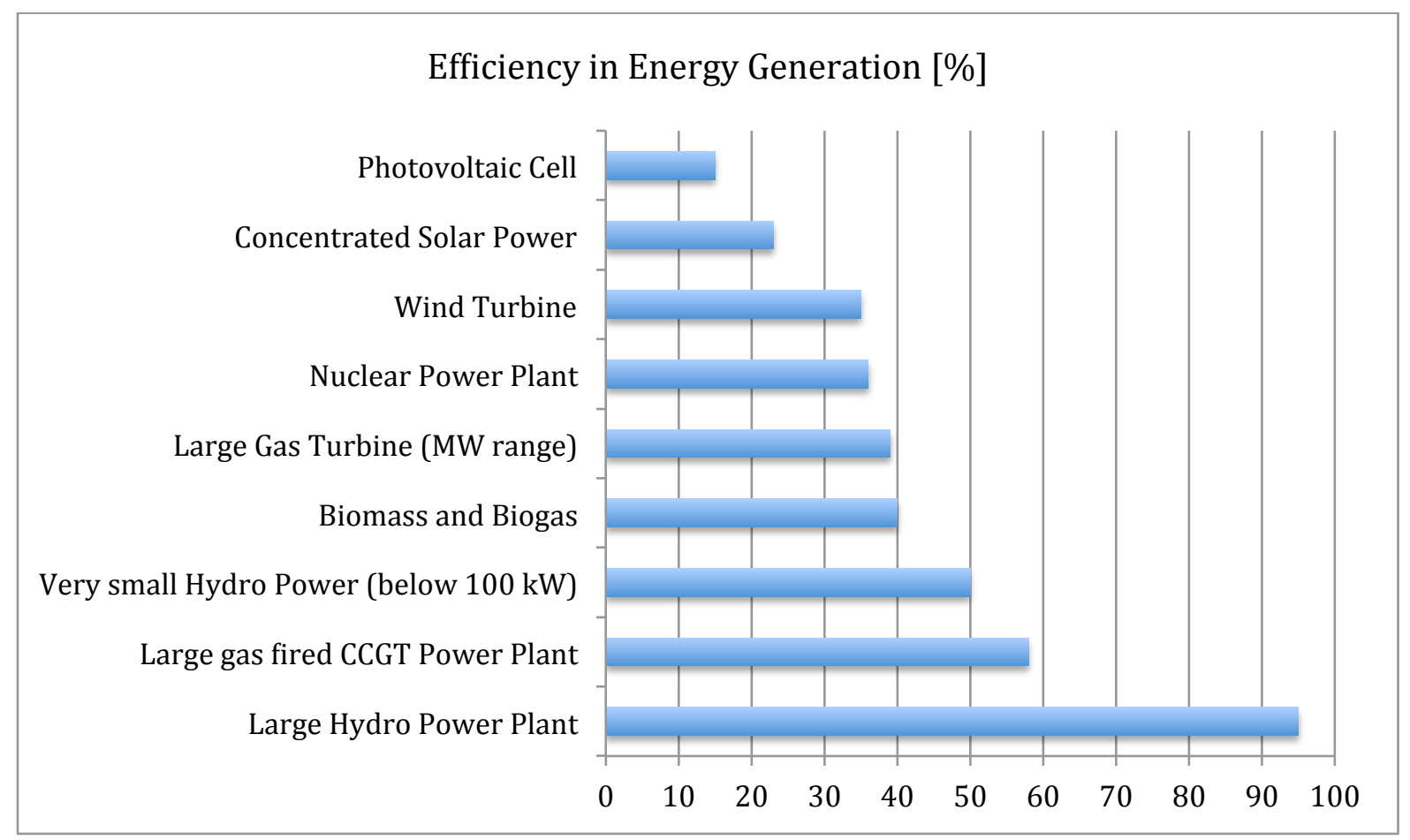

[5.25] Efficiency in Energy Generation (Union of Electricity Industry, 2003: 8-12)

Unfortunately, the efficiency of small hydro-turbines, called pico turbines, with a capacity up to 5 kilowatts $[\mathrm{kW}]$ is much lower, and only a fraction of the theoretical amount of energy available. The losses, compared to the energy transformation, are much higher. But even for a pico hydroelectric setting, the overall efficiency of 50 per cent (which is realistic) would be a

\footnotetext{
${ }^{47}$ In CCGT, power is generated more efficiently than in a simple gas turbine cycle. The hot exhaust gases of the gas turbine are used to produce steam that generates electricity in a steam turbine cycle.
} 
very high efficiency compared to other renewable energy technologies. However, compared to large hydro it would not even have half of the efficiency of a megawatt power station. This is the same principle we have seen in the previous chapter on wind power: efficiency increases with size.

Most hydraulic pumps and turbines are designed and based on preliminary model studies: "The behavior of these models are based on the principles of hydraulic similitude" (European Small Hydro Association [ESHA], 1998: 164). Hydraulic similitude is achieved if the following criteria are met:

○ Geometrical similarity

○ Kinematic similarity

○ Dynamic similarity (Matthias, 2003: 36)

The result of experimental values is typically presented with characteristic figures. These figures help during design calculation. The "specific speed" is one of these characteristic figures, determining the speed of a turbine under the condition of 1 meter of head and 1 cubic meter per second of flow. Any turbine with identical geometric proportions, even if the sizes are different, will have the same specific speed. Different turbine types can be distinguished with regards to their different specific speed: "If the model had been refined to get the optimum hydraulic efficiency, all turbines with the same specific speed will also have an optimum efficiency" (ESHA, 1998: 164). The characteristic figure of specific speed is a useful criterion to select a specific turbine for a specific combination of head and flow.

Chapter 6 will describe characteristics and application ranges of different turbine types. First, in Chapter 5.5, I will provide a comparison of the different technologies presented, along with a feasibility matrix evaluating their applicability for open source rural electrification. 


\subsection{Technology Comparison and Feasibility Matrix}

This chapter offers a comparison of the different renewable energy technologies described

(table 5.26), followed by a feasibility matrix evaluating the different conversion technologies.

The feasibility matrix evaluates selected criteria and multiplies them with weighting factors.

The criteria were selected during the study and cover the main aspects of the available technical solutions, considering technical, economic and organizational aspects.

\section{Comparison table:}

The following table gives an overview of the application range, listing advantages and disadvantages of the selected technologies. This compact illustration is the basis for the feasibility matrix that follows.

\begin{tabular}{|c|c|c|c|}
\hline Technology & Application & Advantages & Disadvantages \\
\hline Wind power & 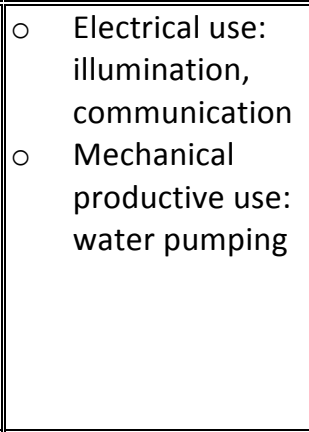 & \begin{tabular}{|ll}
$\circ$ & No costs for fuel \\
$\circ$ & Quick installation \\
$\circ$ & Local production \\
& feasible \\
$\circ$ & Low operational costs \\
$\circ$ & Low investment costs
\end{tabular} & \begin{tabular}{|ll} 
O & $\begin{array}{l}\text { High maintenance (1 or } 2 \\
\text { variances) }\end{array}$ \\
& $\begin{array}{l}\text { Unstable enery supply } \\
\text { (wind changes) }\end{array}$ \\
$\circ$ & Battery bank: expensive \\
& and short lifetime \\
$\circ$ & Proper wind setting \\
& location necessary \\
$\circ$ & DC current and battery \\
storage necessary
\end{tabular} \\
\hline Biogas & \begin{tabular}{|ll} 
O & cooking \\
0 & (electricity)
\end{tabular} & \begin{tabular}{|ll} 
& $\begin{array}{l}\text { Use of organic waste for } \\
\text { electricity generation }\end{array}$ \\
& Production of Charcol
\end{tabular} & $\begin{array}{ll}\circ & \text { Availability of biomass } \\
\circ & \begin{array}{l}\text { Social acceptance } \\
\text { (cooking) }\end{array} \\
\circ & \begin{array}{l}\text { Conversion to electricity is } \\
\text { very complex }\end{array} \\
\circ & \begin{array}{l}\text { Temperature and fuel } \\
\text { availability (if interrupted, } \\
\text { system has to be emptied } \\
\text { and set up anew) }\end{array} \\
\end{array}$ \\
\hline $\begin{array}{l}\text { SHS System } \\
\text { (Photovoltaic) }\end{array}$ & 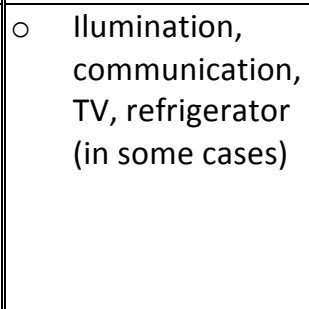 & 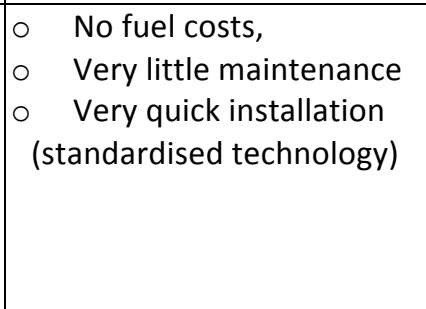 & \begin{tabular}{|ll} 
& Costly replaceable battery \\
bank \\
O & DC voltage \\
$\circ$ & Little energy compared to \\
& investment costs \\
& Battery charging only \\
when there is sun
\end{tabular} \\
\hline
\end{tabular}




\begin{tabular}{|c|c|c|c|c|c|c|}
\hline $\begin{array}{l}\text { Solar Thermal } \\
\text { Power (CDP) }\end{array}$ & O & $\begin{array}{l}\text { Mainly cooking } \\
\text { (electricity) }\end{array}$ & $\begin{array}{l}0 \\
0 \\
0 \\
0\end{array}$ & $\begin{array}{l}\text { No cost for fuel } \\
\text { Simple technology } \\
\text { Fast setup and } \\
\text { installation } \\
\text { Little maintenance } \\
\text { required }\end{array}$ & $\begin{array}{l}0 \\
0\end{array}$ & $\begin{array}{l}\text { Social acceptance } \\
\text { (cooking) } \\
\text { Complex technology for } \\
\text { electricity generation } \\
\text { Energy only when there is } \\
\text { sun }\end{array}$ \\
\hline Hydropower & 0 & $\begin{array}{l}\text { Electrical use: } \\
\text { illumination, } \\
\text { communication, } \\
\text { TV, soundsystem, } \\
\text { refrigerator, etc. } \\
\text { Mechanical } \\
\text { productive use: } \\
\text { grinding, etc. }\end{array}$ & $\begin{array}{l}0 \\
0 \\
0 \\
0 \\
0\end{array}$ & $\begin{array}{l}\text { Long lifetime } \\
\text { Minimal costs } \\
\text { Low maintanence } \\
\text { High reliability } \\
\text { 24-hour AC current, no } \\
\text { battery banks needed } \\
\text { Local manufacturing } \\
\text { possible }\end{array}$ & $\begin{array}{l}0 \\
0 \\
0 \\
0\end{array}$ & $\begin{array}{l}\text { Availability of water and } \\
\text { natural conditions } \\
\text { necessary } \\
\text { Complexity of technology } \\
\text { Accessibility of technology } \\
\text { Long installation time } \\
\text { High maintenance } \\
\text { necessary }\end{array}$ \\
\hline
\end{tabular}

[5.26] Advantages and disadvantages of rural electrification technologies (author's illustration)

We see here that electrification is technically very difficult through biogas and concentrated solar power. On a small scale, these two technologies are successfully applied for cooking. Additionally, the technologies listed above have advantages and disadvantages, which I evaluate using a five-category rating system.

The following feasibility matrix evaluates five renewable energy solutions. This is done through evaluating selected criteria and multiplying them with weighting factors. The criteria were selected during the study and cover the main aspects of available technical solutions, considering technical, economic and organizational aspects. The evaluation of the criteria uses the following five-category rating system (RS):

- $\quad$ RS --: very negative effect

- RS -: negative effect, but not crucial

- RS 0: no significant effect / rather neutral

- $\quad$ RS +: positive effect, but not crucial

- $\quad$ RS ++: very positive effect 
The outcome of the RS is multiplied with weighting factors (WF). Weighting factors are helpful, since there are certain criteria that are more important for the overall project feasibility than others. The weighting factors range from one to three, as follows:

-WF 1: no special focus on the criteria

○ WF 2: important criteria, but not crucial

○ WF 3: high-factor, crucial criteria

The identified criteria are key factors for decision-making regarding the technical approach selected. Financial competitiveness, scalability, energy availability and other uses of energy are less important factors. Energy availability is important, yes. However, renewable energy is free and even if there is only a sun current available during the day, a PV system could be an additional energy source for higher energy use during the day. Technical complexity and maintenance complexity refer to the installation, maintenance and general complexity of the technology. It is very important that the selected technology can be handled without significant effort or serious capacity-building processes. The same is valid for the manufacturing process, which must be as easy as possible. If manufacturing is complex and requires special tools, it might not be possible to reach rural and remote areas, where complex manufacturing facilities are rare.

Another important factor is that the selected technology can be adapted to the DIY approach. This includes the whole process, from the site assessment to manufacturing, installation and maintenance. Technical standardization would be helpful for all of these factors, and would help users create, exchange and share routines that could support each step of the entire process. The most important factors are local access of the energy source and if the technical principle is already available in an open source manner. The local accessibility refers to the energy source. For wind power, a certain wind speed is necessary; solar power requires a sun current, etc. It is crucial that the energy source is available in amounts that can be 
transformed into electrical energy. Every technology has its characteristics and limitations. Similarly, it is important that the technical solution selected exists in open source. If a technical solution has already been developed, used in an open source manner, and/or with a community already working on that topic, it makes most sense to contribute to such a community rather than start a new one. As such, this is also a crucial factor in the technology selection process.

In table 5.27, the combination of WF with RS results in a ranking of available renewable energy solutions.

\begin{tabular}{|c|c|c|c|c|c|c|}
\hline Selected Criteria & WF & Biogas & Wind & PV & CSP & Hydro \\
\hline "Technical Complexity & 2 & - & + & +++ & - & - \\
\hline Manufacturing (local) & 2 & + & ++ & -- & ++ & ++ \\
\hline Financial Competitiveness & 1 & ++ & + & - & + & + \\
\hline Open Source Availability & 3 & - & -- & - & -- & ++ \\
\hline Scalability & 1 & ++ & + & ++ & ++ & ++ \\
\hline $\begin{array}{l}\text { Efficiency of Energy } \\
\text { Transformation }\end{array}$ & 1 & - & 0 & 0 & - & ++ \\
\hline $\begin{array}{l}\text { Local Accessibility of } \\
\text { Energy Source }\end{array}$ & 3 & + & - & - & - & -- \\
\hline Maintenance Complexity & 2 & -- & - & + & ++ & - \\
\hline Energy Availability (24/7) & 1 & ++ & -- & -- & -- & ++ \\
\hline DIY Ability & 2 & + & + & - & ++ & + \\
\hline $\begin{array}{l}\text { Other Use of Energy } \\
\text { (mechanical) }\end{array}$ & 1 & -- & ++ & -- & -- & ++ \\
\hline Technical Standardization & 2 & ++ & ++ & ++ & ++ & -- \\
\hline $\begin{array}{l}\text { Sum (without weighting } \\
\text { factors) }\end{array}$ & - & 4 & 4 & -3 & 2 & 10 \\
\hline $\begin{array}{l}\text { Sum (including weighting } \\
\text { factors) }\end{array}$ & 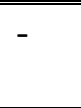 & 5 & 3 & -5 & 3 & 7 \\
\hline
\end{tabular}

[5.27] Technology Selection (author's illustration) 
Hydropower and biogas are ranked best. Solar photovoltaic solutions do not seem appropriate, also because of their low DIY applicability. The crucial categories "Local accessibility of energy source" and "open source availability" are weighted with 3 , and therefore have a heavy influence on the ranking. Wind and CSP are ranked equally, and both seem appropriate as rural electrification technologies. However, they are both limited in their capacity for transforming electrical energy, especially wind power. This study will not cover wind power or CSP, because there are open source wind turbines available already, and although GoSol's focus is cooking, the electrical application will follow in the near future. Biogas appears to have some advantages, but is also a very technically complex solution. The "plant" itself can be built with standard material, very low cost and minimal time investment. However, the chemical process is complex and the gas production depends on many factors, including the working temperature of the bacteria and the "fuel" itself, since there are significant differences as to how much and what kind of excrement is used. Despite its poor performance in "Local Accessibility of Energy Source" and "Technical Standardization", hydropower is ranked best. According to this evaluation, with and without weighting factors, hydropower as a DIY solution for rural electrification appears to be the most appropriate technology.

\section{Synthesis:}

Hydropower is perhaps one of the most complex technical solutions for renewable energy. However, one advantage is that after the installation it runs almost maintenance-free. Its financial range is feasible, it is scalable, and the mechanical form of energy can be used at the same time - for milling grain during the day and charging batteries at night. The overall evaluation of the available technologies for rural electrification ranks hydropower with the most potential. In the following chapter, hydropower as a rural energy solution will be 
evaluated using a SWOT analysis to determine opportunities and threats, as well as the strengths and weaknesses.

\subsection{Hydropower SWOT Analysis}

With the goal of using the energy of water to create electricity on a small scale, this chapter explores the strengths and weaknesses of such a solution, using a SWOT analysis (SWOT stands for Strengths, Weaknesses, Opportunities and Threats). A SWOT analysis is a management tool used to identify an organization's strengths and weaknesses (S-W), as well as broader opportunities and threats $(\mathrm{O}-\mathrm{T})$, and helps to develop a broader awareness of the situation and helps to define a strategy as the basis for an action plan and decision making. (University of Kansas, 2015). It explores the possibilities for new efforts and identifies opportunities for success, evaluating internal factors of an organization, as well as the external factors of the environment.

Strengths and weaknesses are internal factors of an organization and tend to be experienced in the present. The internal analysis for open source hydropower projects is limited in its validity at the moment, since there is no typical hierarchical organization in open source, and at the moment, there is no specific organization at all. at this moment there is no organization at all. Nevertheless, considering these aspects supports the creation of a more holistic view on the endeavor.

A SWOT analysis also elaborates on opportunities and threats, the environmental framework and external factors, which tend to be projected onto the future. The following table depicts the SWOT analysis matrix for open source hydropower as DIY rural electrification 
technology. This table provides an overview of internal and external factors as the basis for deriving and articulating a strategy to form a legal entity and technical design for open source hydropower. The strategy aims to overcome weaknesses and prepare for threats, as well as to maintain strengths and take advantage of opportunities. A strategy is the abstract basis for developing a roadmap and a specific action plan. The defined actions are measured in accordance with the strategy. 


\begin{tabular}{|c|c|c|}
\hline External Factors & $\begin{array}{l}\text { Opportunities } \\
\circ \text { High rural electrification potential } \\
\circ \text { (Open Source) Technology hardly } \\
\text { available } \\
\circ \text { Low investment costs } \\
\circ \text { Timing of benefit through } \\
\text { international OSH movement and the } \\
\text { need for renewable energy } \\
\circ \text { Raising awareness for sustainable } \\
\text { living }\end{array}$ & $\begin{array}{l}\text { Threats } \\
\text { ○ Technical complexity, difficult to } \\
\text { communicate to "consumers" } \\
\circ \text { Complex technology, } \\
\text { standardization } \\
\circ \text { Very limited market segment, low } \\
\text { demand }\end{array}$ \\
\hline $\begin{array}{l}\text { Strengths } \\
\text { - Niche, very small market segment } \\
\text { - Network of Hydropower specialists }\end{array}$ & $\begin{array}{l}\text { SO-Strategies } \\
\circ \text { Establishing a pico hydro-turbine } \\
\text { project in a niche market }\end{array}$ & $\begin{array}{l}\text { ST-Strategies } \\
\circ \text { Develop easily understandable } \\
\text { manuals and documentation } \\
\circ \text { Reduce complexity where possible }\end{array}$ \\
\hline $\begin{array}{l}\text { Weaknesses } \\
\text { - Competition with other renewable } \\
\text { energy technologies } \\
\text { - Financial resources for first design, } \\
\text { prototyping and testing } \\
\text { - No technical testing facility available } \\
\text { - Lack of working force }\end{array}$ & $\begin{array}{l}\text { WO-Strategies } \\
\circ \text { Create clear project documentation } \\
\circ \text { Fundraising and gathering people and } \\
\text { supporters }\end{array}$ & $\begin{array}{l}\text { WT-Strategies } \\
\circ \text { Standardization, as far as possible } \\
\circ \text { Develop easily understandable } \\
\text { manuals and documentation } \\
\circ \text { Integrate network of hydropower } \\
\text { specialists } \\
\circ \quad \text { Install a "Showroom" test-site }\end{array}$ \\
\hline
\end{tabular}

[5.28] SWOT Analysis Matrix for open source Hydropower (author's illustration, 2013) 


\section{Weaknesses / Threats:}

The identified weaknesses and threats concern financial, technical and market issues. To overcome them, different measures must be taken. Financially, it is important to find an initial funding source and a manufacturing and testing facility. Technically, it is important that the documentation is easily understandable and that the complex context is communicated in an appropriate way. At the same time, the design should be standardized, as much as possible, to make it easier to understand. There is very limited market segment, and therefore very low demand for the technology, which is a threat to the project as an external factor that cannot be influenced. However, although the market segment is limited, there is still is a market segment representing a certain level of demand.

\section{Strengths / Opportunities:}

The strengths and opportunities of this technology are the global energy situation and enormous potential for rural electrification. At the same time, the unique selling proposition of starting a community in the field of hydropower is a very special situation. Given that investment costs are low regarding time and money, the overall project opportunity appears attractive. Also, the opportunity for joining the special dynamics of the OSH movement could contribute to the rapid growth and development of such a project. In such a niche market, at a time where awareness for sustainable living is growing, it appears to be an ideal moment for sharing a sustainable technology for decentralized electrification under an open license.

From the SWOT analysis, the following strategies were developed:

\section{$\underline{\mathrm{SO}}$ - Strategies}

Establish a pico hydro-turbine project in a niche market 


\section{$\underline{\text { ST - Strategies }}$}

- Develop easily understandable manuals and documentation

- Reduce complexity where possible

WO - Strategies

- Create clear project documentation

$\circ$ Fundraising and gathering people and supporters

\section{WT - Strategies}

- Standardization, as far as possible

- Develop easily understandable manuals and documentation

- Integrate network of hydropower specialists

○ Install a "Showroom" test site

\subsection{Conclusion}

According to the technology comparison table, the feasibility matrix and the hydropower SWOT analysis, hydropower seems to be an appropriate technology for the open source approach. It is clear that hydropower represents a rather complex field of mechanical engineering, requiring extensive knowledge to build, run and maintain such a machine. However, given hydropower's enormous potential and low investment efforts, it is feasible to form an open source hydropower group and start designing a Pico Hydro-Turbine.

The following chapter investigates which of the turbine types are the most appropriate for this endeavor. 


\section{Feasibility of an Open Source Hydro Turbine}

The outcome of the comparison matrix demonstrates that hydropower is a feasible option for rural electrification. The SWOT analysis offered additional perspectives on hydropower as a useful rural electrification solution. This chapter investigates the technical options for different types of hydro-turbines, evaluating for functionality, complexity, manufacturing processes and financial aspects.

\subsection{Turbine Types and Technical Evaluation}

There are several ways to classify the different types of hydro-turbines. According to their fundamental working principle, hydraulic turbines are classified into two groups - impulse turbines and reaction turbines. Impulse turbines are those in which the water hits the turbine blade at atmospheric pressure, and the turbines work with the impulse of water. In this case, the water pressure is completely converted into kinetic energy before entering the runner "The kinetic energy is in the form of a high-speed jet that strikes the buckets, mounted on the periphery of the runner" (ESHA, 1998: 164). After striking the buckets of the runner, ideally the water falls into the tail water with only a little bit of remaining potential and kinetic energy. Therefore, the housing for impulse turbines can be light and serve mainly to prevent splashing. Pelton and Michell Banki (Cross Flow) turbines are impulse turbines.

In reaction turbines, the water pressure applies a force on the runner blade that decreases as it proceeds through the turbine. The British Hydropower Association (BHA) has formulated the following explanation: "Reaction turbines exploit the oncoming flow of water to generate hydrodynamic lift forces to propel the runner blades" (BHA, 2012: 28). The runner is fully 
immersed in water, and the water is directed by movable guide vanes. As such, the turbine casing must be strong enough to withstand the operating pressure. Kaplan and Francis turbines are examples of reaction turbines. Hydraulic pumps also work with the reaction principle and, if turning backwards, can be used as turbines. Reaction turbines "have a diffuser known as a 'draft tube' below the runner through which the water discharges. The draft tube slows the discharged water and so creates suction below the runner which increases the effective head" (BHA, 2012: 28). The working principle of impulse and reaction turbines is visible in illustration 6.1. On the left, we see the horizontal axis Kaplan turbine, a reaction turbine, completely submerged in water. The Pelton turbine on the right side is impacted by a small stream of water and runs in the air.

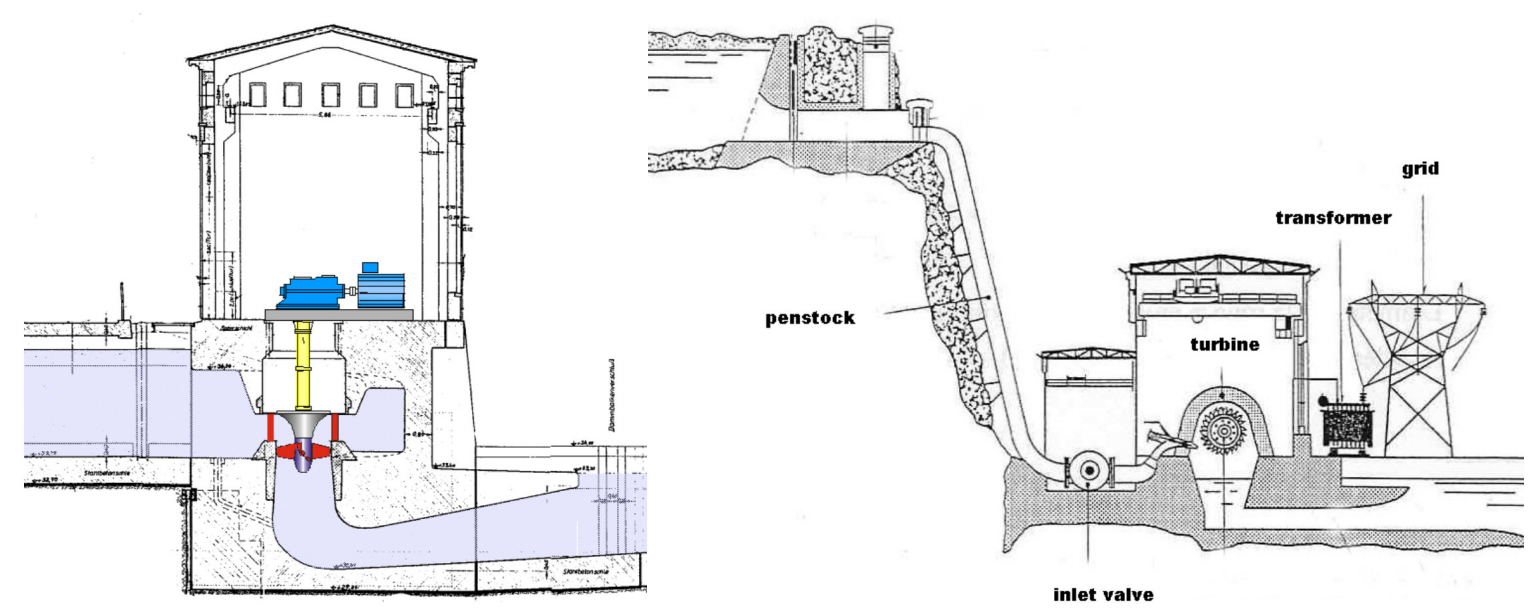

[6.1] Schematic view of a low and high head power house (ESHA, 2008: 155)

Another more widespread classification of hydro-turbines is according to the height and flow range, referring to the characteristics of the environment in which they can be used, as follows:

○ High head and small flow hydraulic turbines

- Medium head and medium flow hydraulic turbines

○ Low head and high flow hydraulic turbines 
The following table 6.2 illustrates the aforementioned classification criteria, operation type and application range, in one table. This illustration is very useful for a initial step of defining an appropriate turbine type for a hydropower site.

\begin{tabular}{|l|l|l|}
\hline Type of Operation & Type of turbine & Head and Flow \\
\hline \hline \multirow{5}{*}{ Action } & $\begin{array}{l}\text { Pelton } \\
\text { Turgo }\end{array}$ & High head and little flow \\
\cline { 2 - 3 } & $\begin{array}{l}\text { Michel Bank / Cross Flow } \\
\text { Pelton (with multiple } \\
\text { injectors) }\end{array}$ & $\begin{array}{l}\text { Medium head and medium } \\
\text { flow }\end{array}$ \\
\hline \multirow{5}{*}{ Reaction } & $\begin{array}{l}\text { Francis } \\
\text { Pumps as Turbines }\end{array}$ & \\
\cline { 2 - 3 } & $\begin{array}{l}\text { Propeller } \\
\text { Kaplan }\end{array}$ & Low head and high flow \\
\hline
\end{tabular}

[6.2] Classification of Turbine Types (author's illustration 2014)

\section{Turbine selection:}

Each turbine has certain advantages and disadvantages to consider, and selecting the best turbine for any particular site depends on the characteristics of the site, particularly the head, flow and power demand. These classifications are useful for initiating the selection process; however, a more precise turbine selection for a given site can be made by evaluating the specific speed. According to laboratory test results, every turbine has a specific speed range where it works at optimum efficiency. The concept of specific speed is mentioned often in hydropower texts, and it is a useful value for turbine classification. The specific speed required for a site can be calculated as follows (Raabe, 1985: 326):

$$
N S=N \cdot \frac{\sqrt{P}}{H^{\frac{5}{4}}} \quad \begin{array}{ll}
\text { with: } \\
\text { Ns }=\text { Specific Speed } \\
\mathrm{N}=\text { Nominal Speed } \\
\mathrm{P}=\text { Power } \\
\mathrm{H}=\text { Height }
\end{array} \quad \begin{aligned}
& {[-]} \\
& {[\mathrm{kWm}]} \\
&
\end{aligned}
$$


This equation expresses the relationship between head, turbine output power and actual turbine speed, and is applicable to all turbine types. According to Harvey (1993), the specific speed is defined as follows:

Specific speed is a constant which describes the particular machine as fabricated, running at its design/peak efficiency, and has a value which con only be supplied by the turbine manufacturer. Larger or smaller turbines of the same shape would have identical specific speed (Harvey, 1993: 160).

The specific speed of various turbine types is visible in illustration 6.3. For high head turbines the specific speed has a low value, and for low head turbines the specific speed has a high value. With the field measurements of the particular site, it is possible to calculate the specific speed. Ascertaining the specific speed makes it easy to find an appropriate turbine for the site. Unfortunately, manufacturers of micro and pico turbines do not provide information on the specific speed of their turbine models. This is perhaps due to cost and lack of access to a fluid dynamics laboratory, where turbines can be tested to determine specific speed. However, the specific speed is a good initial indicator for determining what kind of turbine is required for particular site conditions.

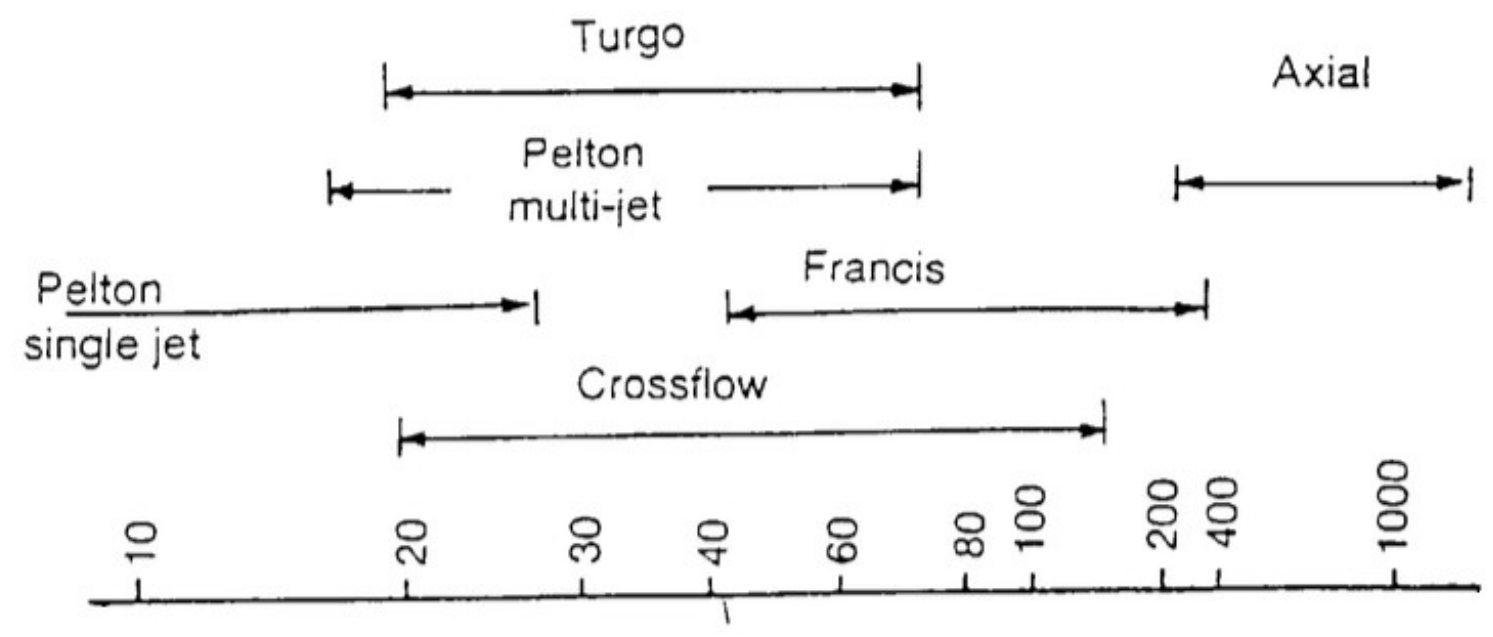

[6.3] Specific Speed for various turbine types (Harvey, 1993: 156) 
Another way to select the appropriate turbine type is according to height and flow. The following graph [6.4] depicts the application ranges of different turbine types in relation to the head and flow, visible on the two axes. The figure is based on large hydropower plants, so the flow rates depicted are rather high. In pico hydro, the flow rates are much lower; therefore, the energy output is much lower, and the dimensions of the turbines are smaller. According to the hydraulic similitude, turbine dimensions are scalable. Therefore, the application range for different turbine types is also applicable for pico hydropower.

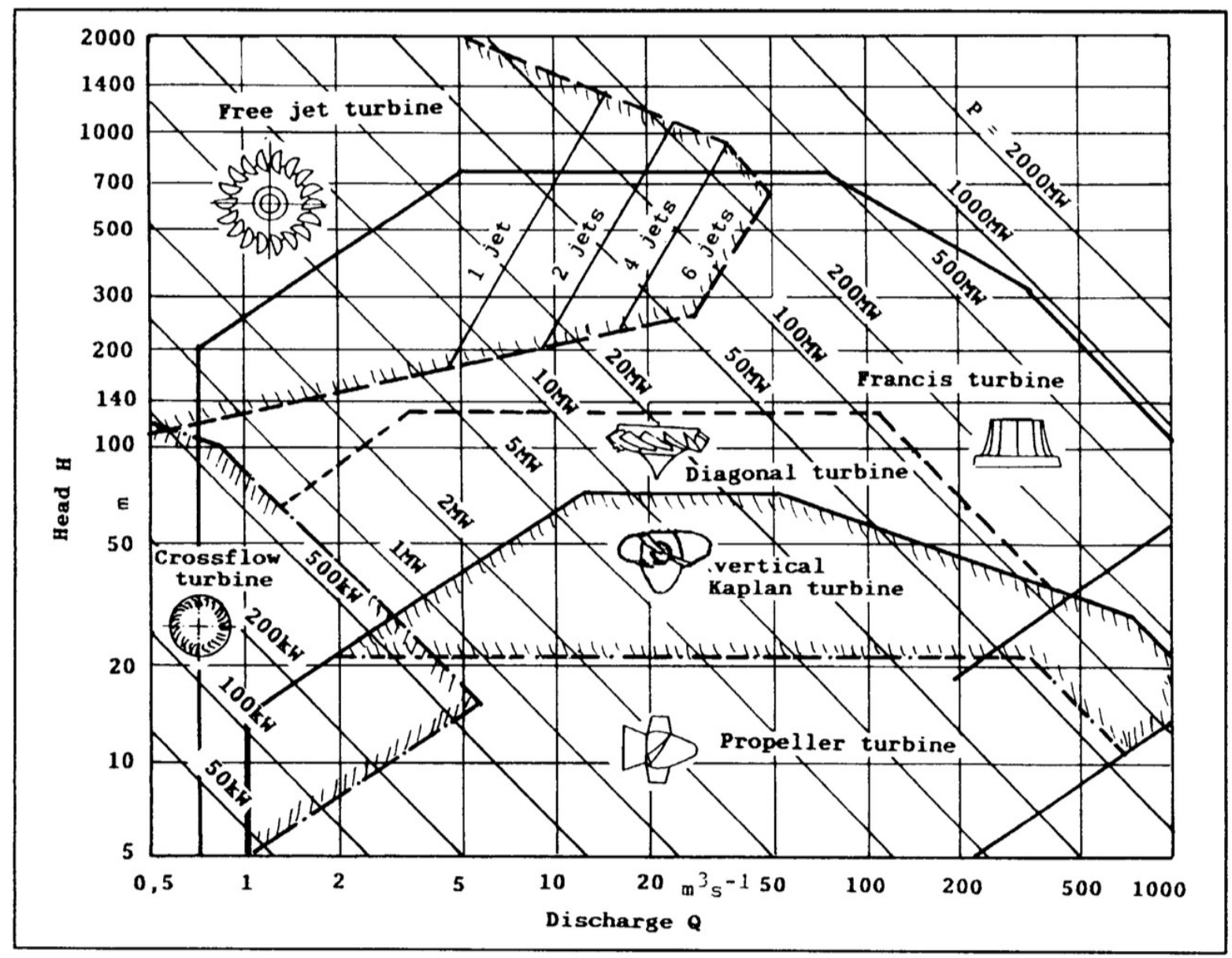

[6.4] Functional Range of different Water Turbines by Escher-Wyss (in Eisenring, 1991: 2)

As we can see, Kaplan turbines are at a high flow and at low heights; therefore, they are also called low head turbines. Michell Banki turbines are more flexible in flow and height and are applicable in a broader range. The same is valid for Francis turbines, which operate best at slightly higher heads. Unfortunately, Francis turbines have limited availability for pico hydro 
applications, and are more difficult to manufacture where DIY solutions are concerned. For pico hydro and DIY applications, the technology of "pumps as turbines" (PAT) is used. For less flow at high head, the Pelton turbine is applicable. Pelton and Banki turbines are impulse turbines, which work with the impulse, or momentum, of moving water.

Other turbine selection methods - monograms, for example - are not explored within the frame of this research, since the focus here is not on turbine selection techniques. With the methods mentioned here, it is possible to select the appropriate turbine for a particular site.

Selecting the appropriate turbine for a specific site requires knowledge on the turbine's characteristics. The following pages present research on the overall efficiency of a hydroelectric scheme and the specific efficiencies of the different turbine types.

\section{Part Flow Efficiency:}

A significant factor in the comparison of different turbine types is their relative efficiency, both at their design point and at partial flow. In general, turbine manufacturers provide the efficiency curves associated with each turbine. The curve describes the relationship between the flow and the efficiency under a certain or design head.

Graph 6.5, borrowed from the Colorado Energy Office (CEO), presents the typical turbine efficiencies for different turbine types. In this diagram, we see how each turbine will perform under specific conditions. A Cross Flow turbine will not reach efficiencies as high as a Francis or double-regulated Kaplan turbine. This is valid for the best operation point, at 100 per cent of design flow.

In rural electrification in the Global South, the typical use case does not guarantee the availability of the design flow throughout the year, since these regions are often exposed to 
periods of rain and dryness. For that reason, the part flow efficiency is very important, perhaps even more so than the efficiency of the best operating point. "Generally, a flatter efficiency curve represents a turbine that can operate under broad ranges of head and flow. Curves that are steeper and narrower are indicative of a turbine designed for more focused ranges of operation" (CEO, n.d.: 40).

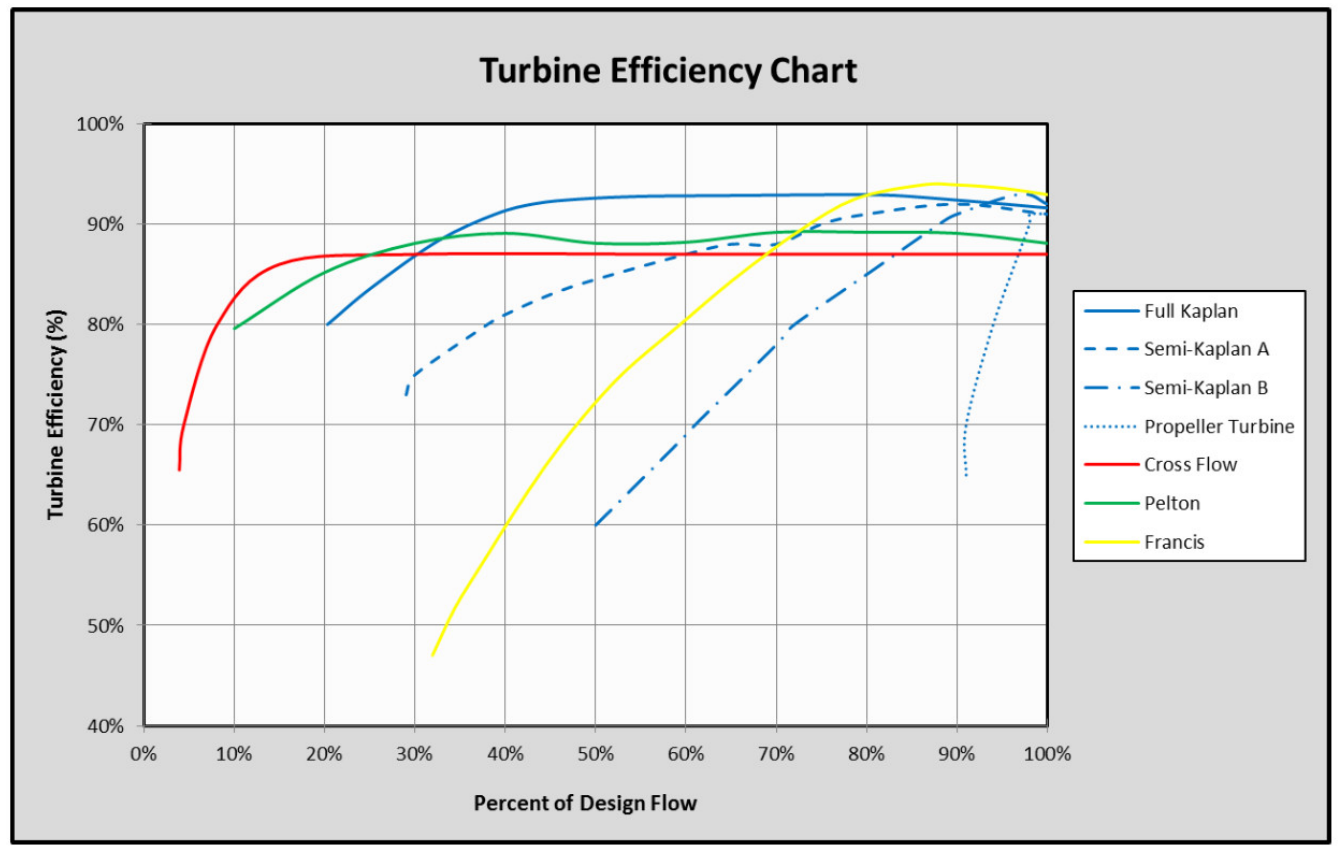

[6.5] Typical Turbine Efficiency by Type ${ }^{48}$ (CEO, n.d.: 40)

An important point to note is that the Cross Flow, Turgo ${ }^{49}$ and Pelton turbines retain very high efficiencies when running below design flow. The Cross Flow turbine's efficiency is almost stable to as low as 20 per cent of the design flow. The performance of the Pelton turbine shows similar behavior, whereas in contrast, the efficiency of the Francis and Propeller turbines falls away more sharply if run at below half their normal flow. Unfortunately, "propeller turbines with fixed guide vanes and blades can operate

\footnotetext{
${ }^{48}$ Full Kaplan, Semi-Kaplan A and Semi-Kaplan B are axial turbines with "regulation". Guide vanes, runner blades or both (double regulated) are regulated according to the flow. These technical, more complex and more costly solutions are not applicable for pico hydro. In pico hydro the Propeller Turbine chart applies instead of the Kaplan chart.

${ }^{49}$ Although not covered in this chart, the performance of the Turgo turbine is similar to the Pelton turbine, as visible in the micro hydro design manual (Harvey, 1993: 156)
} 
satisfactorily only over a very limited range close to their rated discharge" (ESHA, 1998: 179). From the perspective of the use case and the part flow efficiency, impulse turbines seem appropriate for rural electrification in the Global South.

The following subchapters investigate the working principles of the five turbine types (Propeller / Kaplan, Cross Flow, Pumps as Turbines (Francis), Turgo and Pelton), including Functionality, Complexity, Efficiency, Manufacturing and Maintenance. This chapter closes with a comparison table of the different turbine types and a concluding paragraph suggesting the appropriate turbine type for use in an open source hardware project for rural electrification.

\subsubsection{The Propeller and Kaplan Turbines}

Propeller and Kaplan turbines are axial-flow reaction turbines, generally used for low heads and high flow rates, which is the most suitable design for large, slow-running rivers. Designed to work fully submerged in water, they are similar in form to a ship's propeller, but as turbines they rotate in the reverse direction. Victor Kaplan, an Austrian engineer, invented the Kaplan turbine by adding adjustable blades to the propeller-shaped runner and guide vanes, adopted from the Francis turbine to optimize the flow direction of water. The Kaplan runner looks like a propeller, as depicted on the following page (Tiemersma \& Heeren, n.d.: 36).

However, the difference between the Kaplan turbine and the Propeller turbine is that the Kaplan turbine has adjustable blades and often also has adjustable guide vanes. If both the blades and guide-vanes are adjustable, is the turbine is described as "double-regulated". If one of the two, particularly the runner blades, is fixed, it is "single-regulated". Unregulated propeller turbines are used when both flow and head remain practically constant. Usually 
three to six blades are used; three are used in the case of very low head units (Harvey, 1993: 179). The difference between a Propeller turbine and a Kaplan is its moving blades and moving guide vanes. In general, designs are optimized for a particular flow rate, and efficiencies drop off rapidly if the flow rate falls below the design rating. This can be minimized by adjusting runner blades and guide vanes through an electromechanical automatic governing system. The adjustable vanes and blades enable the turbine to work efficiently over a range of flow rates. Various positions of the rotor blades adjusted to accommodate different flow are visible in the schematic drawing of a Kaplan runner (left side: low flow operation; right side: high flow operation).
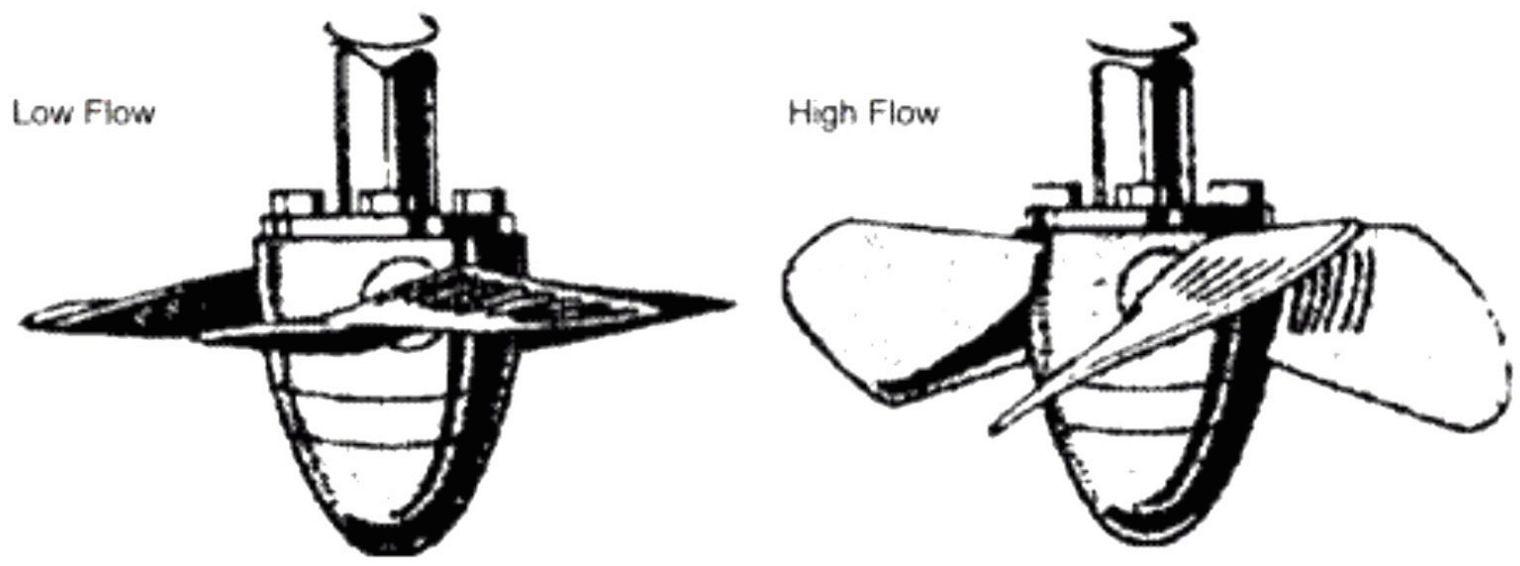

[6.6] Kaplan Turbine with different Blade Angles (Tiemersma \& Heeren, n.d.: 36)

Additionally, a Kaplan hydroelectric scheme could also have adjustable guide vanes: "The Kaplan Turbine is highly adjustable, in both the pitch of the runner blades as well as the inlet guide vanes. This adjustability increases efficiency and allows for a larger flow operating range" (CEO, n.d.: 40). These double regulated designs are rather complex and therefore not used in pico hydropower. The Kaplan turbine has the same setting as the Francis turbine. These vertical axis turbines are surrounded by a scroll case, as visible in Illustration 6.1 and explained as follows: 
The flow enters the case radially inward before being deflected axially and passing through the runner. Because flow is parallel to the shaft, this turbine is also referred to as an axial flow turbine.... The water under pressure enters the turbine through a series of angled guide vanes designed to induce a tangential or whirl component of velocity. On passing through the runner, water pressure is reduced as energy is imparted to the runner. The water then passes on to the tailrace through a draft tube, which recovers some of the remaining head (Inversin, 1986: 182-183).

\section{Technical Complexity and Efficiency:}

The advantage of an axial turbine is that there is no penstock or complicated civil works necessary. The application range is up to about two meters of height difference. For a large amount of flow, a damn is necessary, which usually is a much bigger and cost-intensive construction than the intake of a high head turbine. It has to handle much more water and might be the most expensive part of the hydroelectric scheme.

As mentioned previously, the part flow efficiency of a fixed blade axial turbine drops significantly and can be seen as very poor. This has to be taken into consideration, especially for rural electrification in the Global South, where flow varies throughout the year.

\section{Fabrication:}

The manufacturing of an axial turbine can be done simply in a standard workshop without the use of special sophisticated tools. Some welding is necessary, mainly for the spiral case, and a standard lathe, accompanied with some basic cutting and bending of metal sheets, is enough. The following picture 6.7 depicts an "Axial Simplex" turbine fabricated in León, Nicaragua. The expected power output is up to 5 kilowatts. Although manufacturing is possible, it is complex and technically challenging. As a result of that, axial turbines are not in use with smaller hydropower schemes. This is rooted in their more complex design, which 
makes it much more expensive. Consequently, the turbine used for low head sites is the Cross Flow turbine (Inversin, 1986: 185).

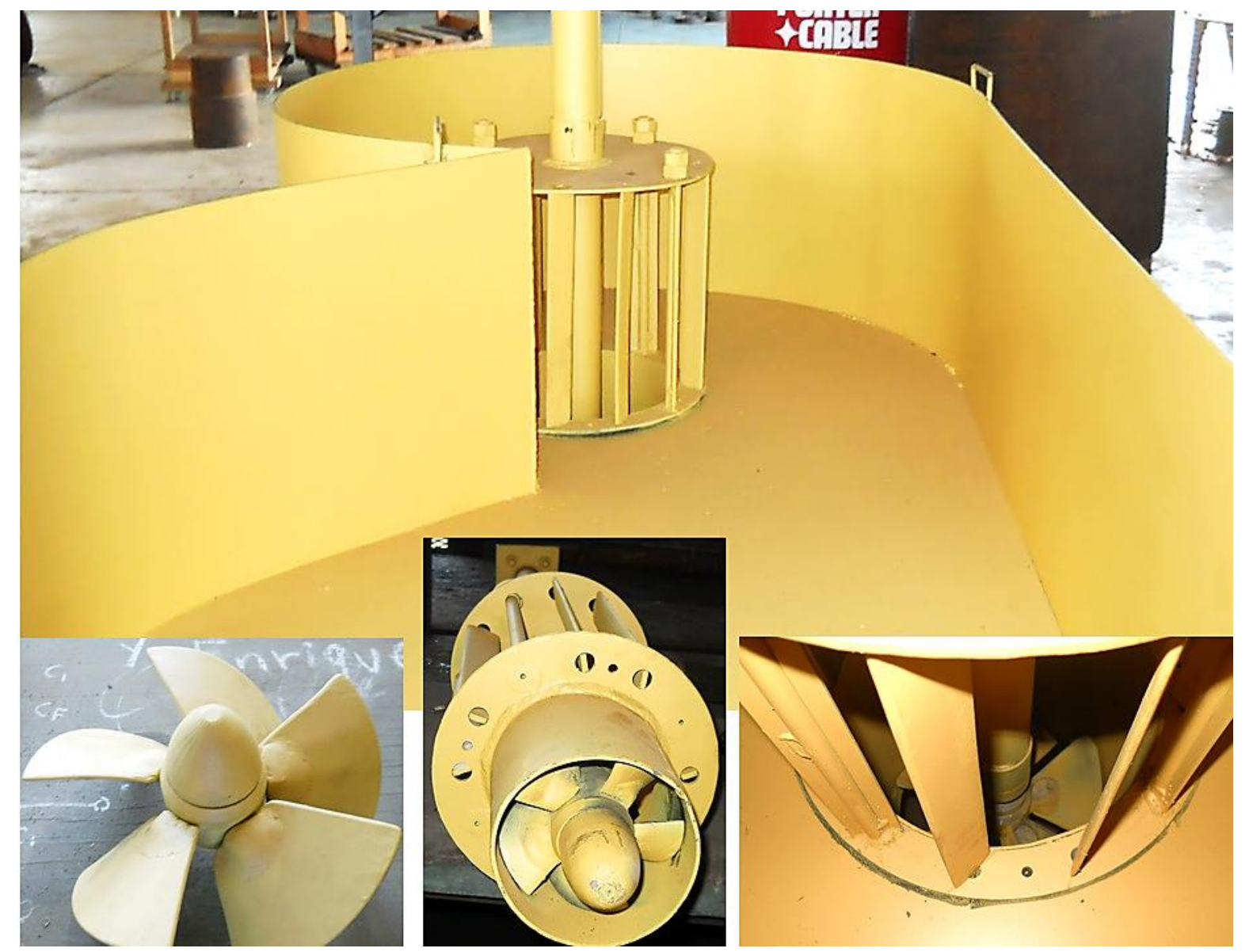

[6.7] Axial turbine fabricated in León, Nicaragua (author's photos)

\section{Installation and Maintenance:}

The most critical part of axial turbines is the need for civil works. The necessary dams may have significant impact on the environment; cutting off fish routes, for example. However, for very low head settings, Kaplan and Banki turbines are the only technical options. Apart from the civil engineering and construction required, the installation of an axial turbine is possible without serious effort. A penstock is not required, which is often a labor-intensive installment job. In the following pictures 6.8 , the installation of a locally manufactured one kilowatt "Simplex" turbine is illustrated. This turbine is used for lighting and as the outdoor laboratory for students of Polytécnico La Salle, León. 

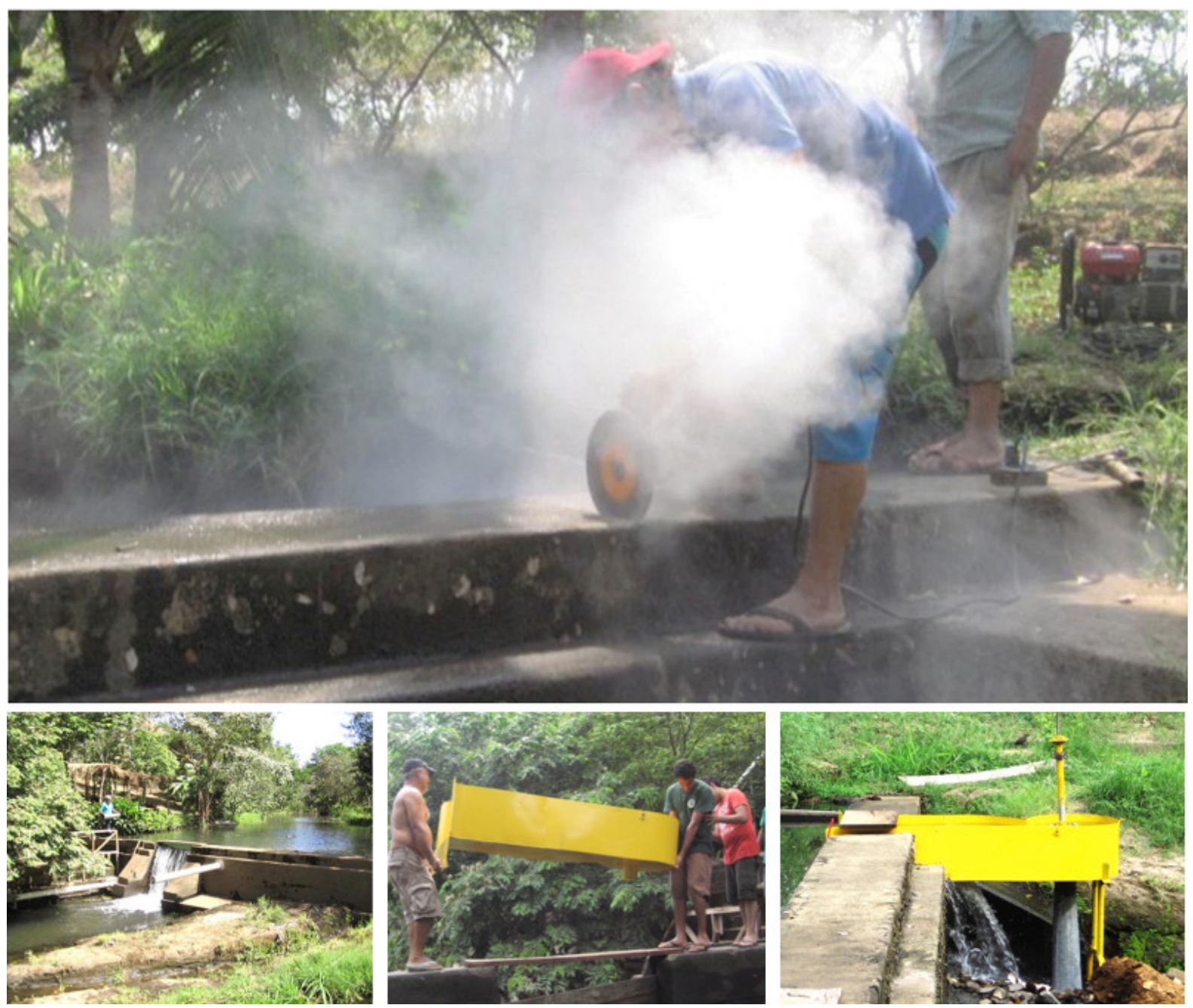

[6.8] Installation of an axial "Simplex" in León, Nicaragua 2012 (author's photos)
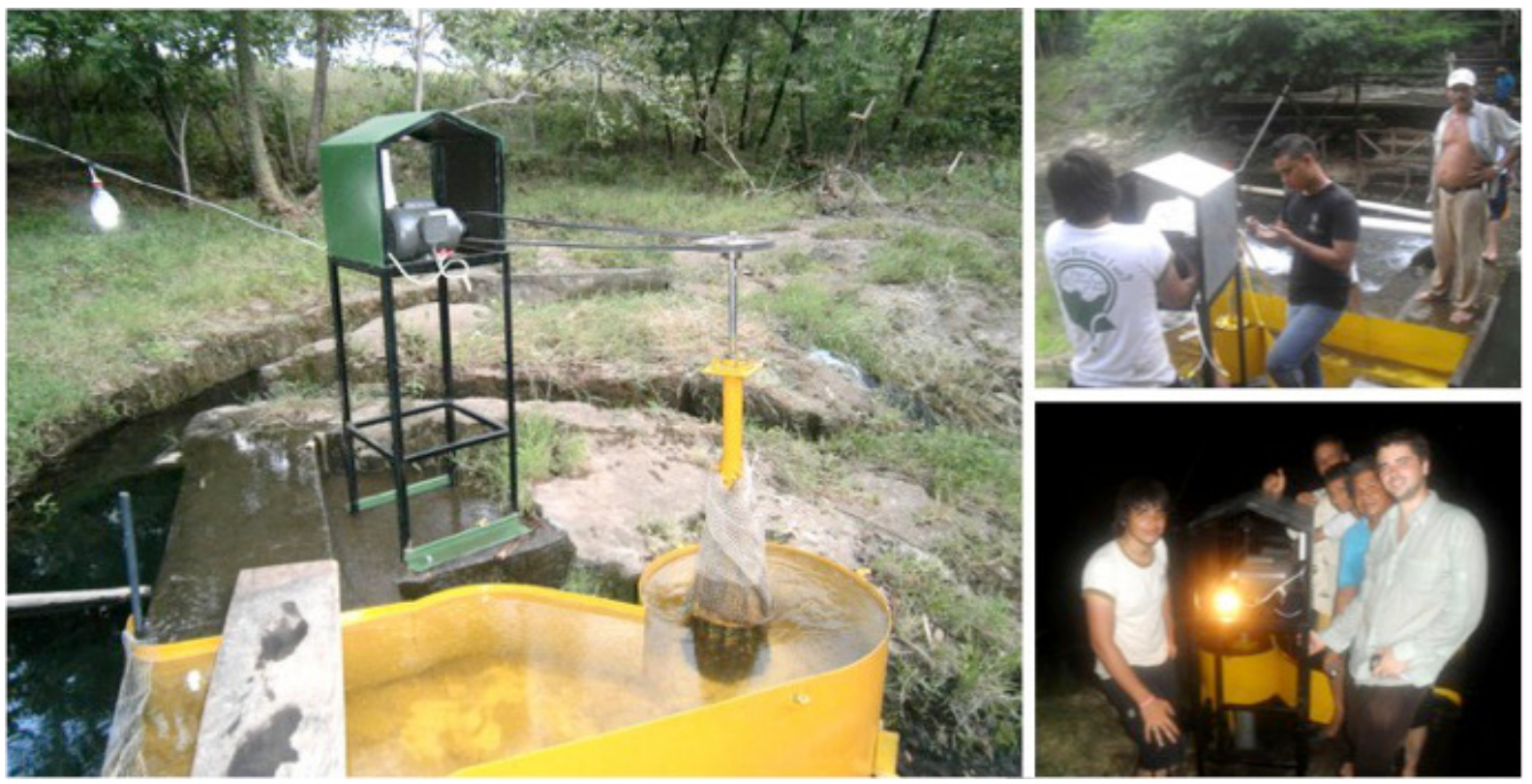


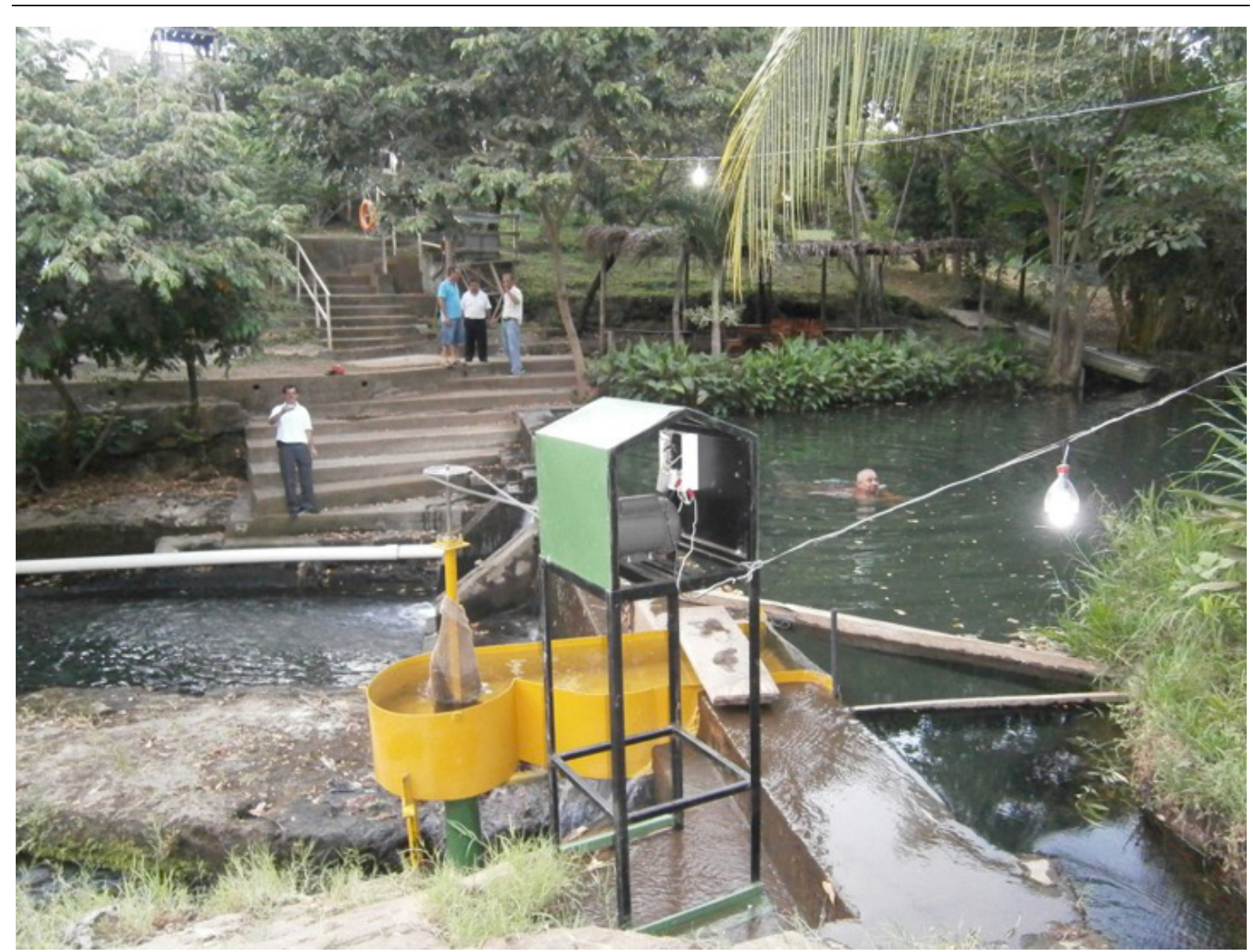

[6.9] Installation of axial "Simplex” Turbine in León Nicaragua 2012, (author's photos)

The difficult part of this turbine type is the maintenance. The turbine has to be protected from all kinds of trash, wood and other surprises that travel down the river. In this specific installation, a heavy rain caused a serious increase of flow and a tree floating down the river washed away the small motor, which was then found several hundred meters down-river. Luckily, it was not damaged and could be reinstalled after drying. Secondly, the "Simplex" design is not a market-ready product. The bearing, for instance, is a weak point and needs to be exchanged frequently. Overall, it is a great installation for renewable energy students in León, and is used as a hands-on outdoor hydro laboratory. 


\subsubsection{The Cross Flow / Banki Turbine}

"The development of the cross flow turbine has been credited to both Banki and Michell, and it has been manufactured commercially for over half a century by the Ossberger Turbinenfabrik in Germany. It therefore commonly goes by the name of the Banki, Michell, or Ossberger turbine" (Inversin, 1986: 178). The Cross Flow turbine is an impulse turbine. The name "Cross Flow" from the turbine's working principle, because the direction of the water is across the runner (CEO, n.d.: 41). The water is directed by one or more guide-vanes, located in a transition piece upstream of the runner. Harvey described the runner design as "the drum-shaped runner of a crossflow turbine is built of two parallel disks connected near the rim by a series of curved blades" (Inversin, 1986: 178). The flow enters in through a rectangular nozzle, rather than a round jet, and flows across the openings between the curved blades" (BHA, 2005: 28). Allen Inversin describes the waterflow as follows: "It then passes through the center of the runner and strikes the buckets a second time as it leaves, imparting a smaller amount of energy before dropping into the tail water" (Inversin, 1986: 178). The more complex flow path through the Cross Flow turbine results in a lower efficiency as compared with other impulse turbines.

The described path of the water and its working principle are depicted in sketch 6.8. On the left side, a section view of a turbine including the flow of water is depicted. On the right side, we can see the runner of a Cross Flow.

Unlike Pelton and Turgo turbines, which can have horizontal and vertical orientation, the Cross Flow is always installed with the shaft positioned horizontally. The photo 6.10 below shows a basic Cross Flow turbine design manufactured in Mozambique as part of a workshop of the multinational development program Energising Development (EnDev $)^{50}$.

\footnotetext{
${ }^{50}$ Energising Development (EnDev) is an energy access partnership currently financed by six donor countries: The Netherlands, Germany, Norway, Australia, United Kingdom and Switzerland. EnDev promotes sustainable
} 

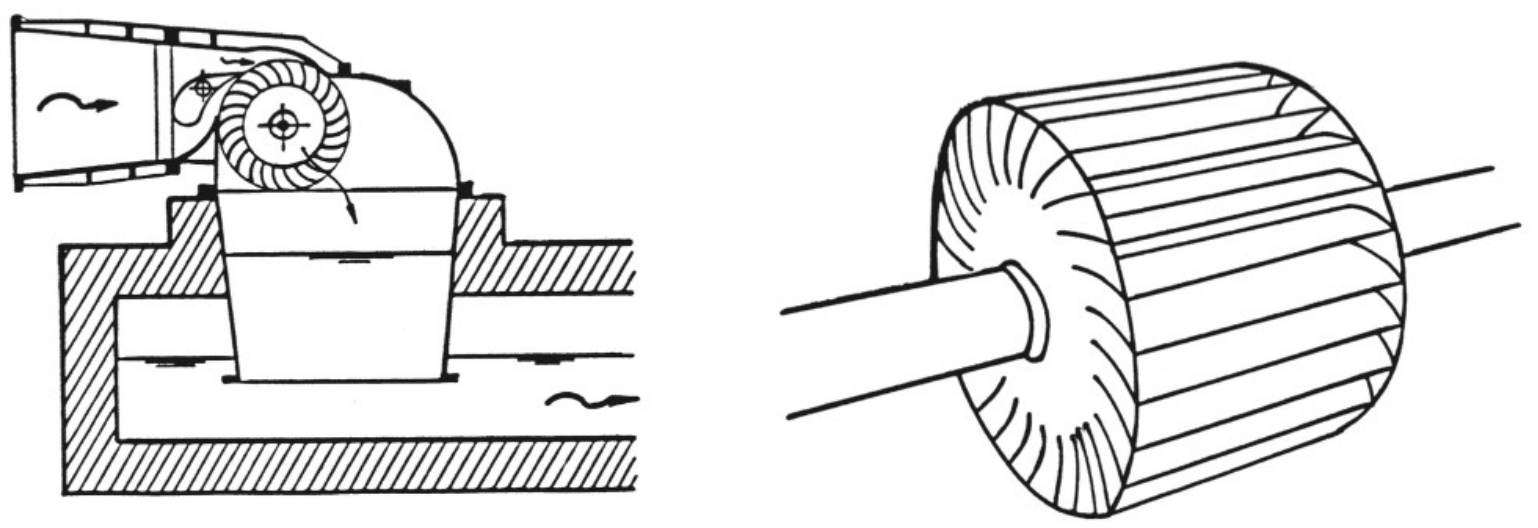

[6.10] Cross Flow working principle; left: turbine section, right: runner (Bundesamt fuer Kulturfragen, 1995: 29)
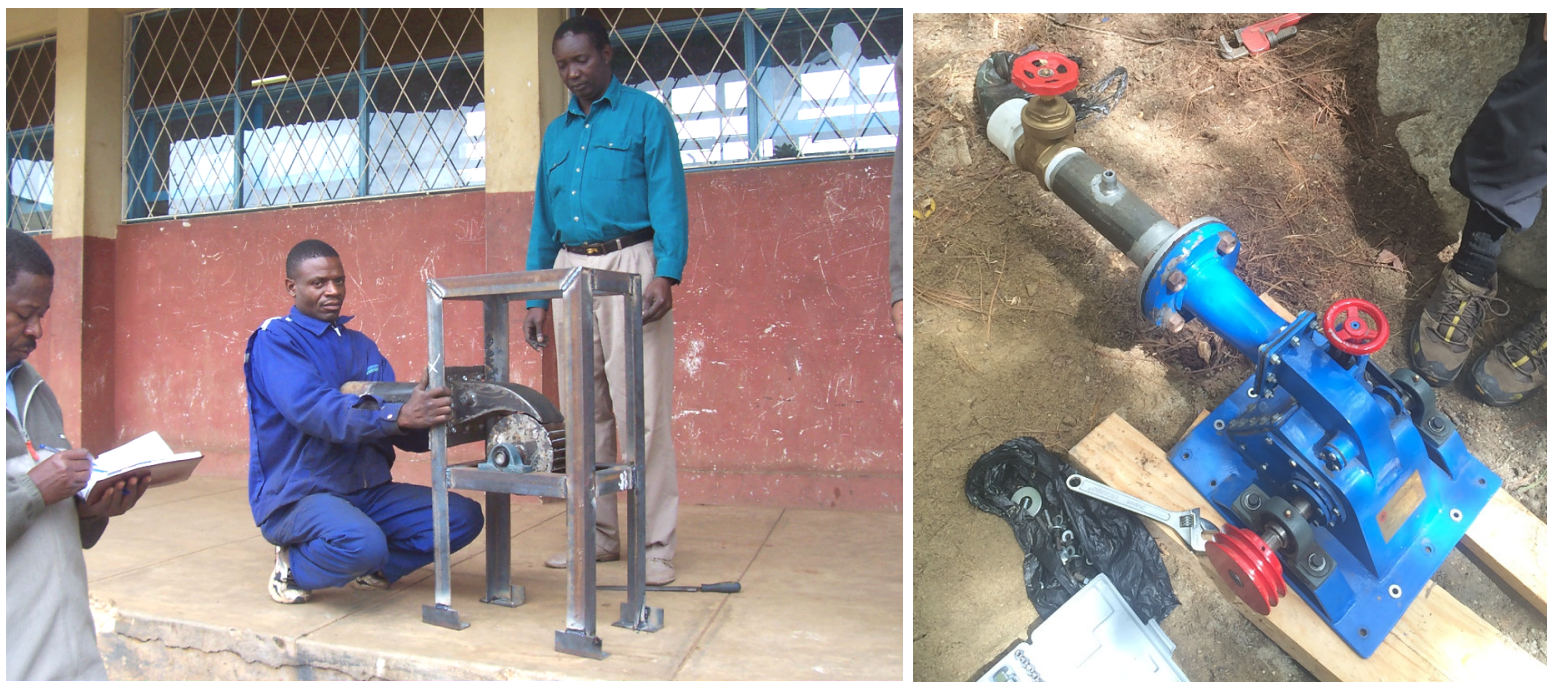

[6.11] Michell Banki Turbine fabrication in Mozambique (Gnecco, 2012)

[6.12] Michell Banki Turbine fabricated in Peru, installed in Nicaragua (Rodriguez, 2015)

A principle advantage of the crossflow turbine is that, because of the symmetry of its blades, the runner width theoretically can be increased to any value without changing the hydraulic characteristics of the turbine. Doubling the runner width for example, merely doubles the power output at the same speed, because the diameter remains unchanged (Inversin, 1986: 179).

As we have seen in the introduction to hydro turbines already the Cross Flow turbine is designed to accommodate large flows in a wide operation range of net head, "spanning from users (Endev, 2015). 
approximately 7 meters to 200 meters of head" (CEO, n.d.: 41). This means that it covers a wide range of possible hydropower sites. "Their efficiency is lower than conventional turbines, but remains at practically the same level for a wide range of flows and heads (typically about 80\%)" (ESHA, 1998: 158).

\section{Technical Complexity and Efficiency:}

The Cross Flow turbine consists of a rather simple principle, perhaps the simplest of hydro turbines. However, the overall turbine and system design can be more elaborate and complex in detail (separate chamber guide vanes, etc.). Especially for larger turbines, we find much more elaborate designs, which has a significant effect on the cost and efficiency of the turbine: "The efficiency of a crossflow depends on the sophistication of its design. Sophisticated machines attain efficiencies as high as 85 per cent. Simpler ones range typically between 60 and 75 per cent" (Harvey, 1993: 174).
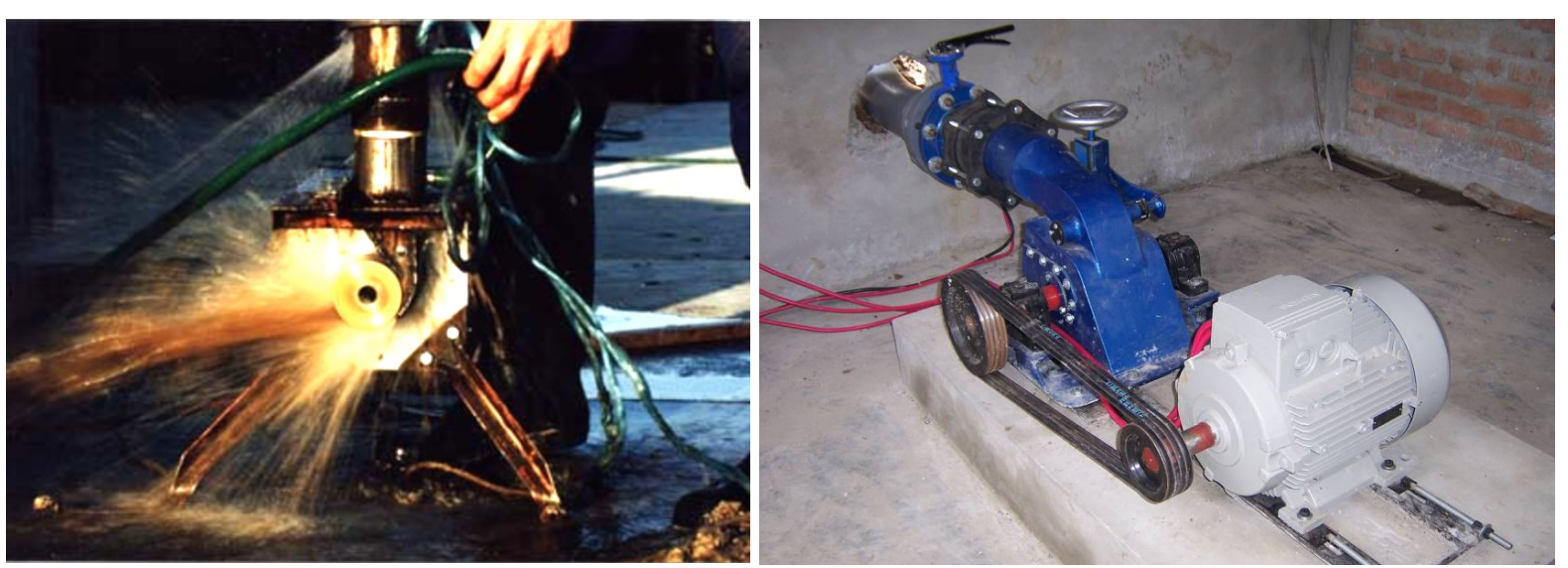

[6.13] / [6.14] Two Cross Flow designs; left: Firefly (Microhydropower, 2015). Right: Cross Flow Installation in Peru (Gnecco, 2012)

In the pictures shown above, two Cross Flow designs are visible: on the left side, an open "firefly" design; on the right side, a Cross Flow turbine designed and manufactured in Peru. 
As previously discussed, the Cross Flow turbine has a lower efficiency than most other hydro turbines. At the same time, it has the significant advantage, of part flow efficiency, which is important especially in the Global South, where the seasons affect the amount of water available: "As evident from the efficiency curve, the Cross Flow is able to maintain a consistent efficiency over a broad range of flow, making it well suited for seasonally fluctuating flow sources" (CEO, n.d.: 41).

\section{Fabrication:}

The runner can be built from two or more parallel disks, connected near their rims by a series of curved buckets or blades. For the buckets, a standardized pipe can be used and cut into appropriate pieces. The disks and buckets have to be welded together. By doing so, only a few tools are needed for the manufacturing of the runner: "Because of the simplicity of its design and the fact that the construction of the runner requires only cutting, welding and grinding (or machining, if available), the cross flow turbine is the most popular turbine for local fabrication" (Inversin, 1986: 180). According to Boyle, who stated: "some ingenious technological ideas have gone into the development of simple types which can be constructed and maintained without sophisticated engineering facilities and are therefore suitable for remote communities" (Boyle, 2004: 171).

Please find additional and more detailed information about design and manufacturing of a Cross Flow turbine in Chapter 7, Practical Application: Pico Cross Flow.

\section{Installation and Maintenance:}

The installation of a Cross Flow turbine is exactly the same as for a PAT, Turgo or Pelton turbine. An intake is necessary, mostly consisting of a small dam und some sediment 
protection according to the site's water condition. The scheme then consists of a penstock, the turbine house with its electromechanical equipment, and the transmission and distribution lines.

The following pictures 6.15 and 6.16 illustrate the installation of a $2 \mathrm{~kW}$ Cross Flow turbine in Ocotal, Nicaragua. The turbine was manufactured in and imported from Perú.
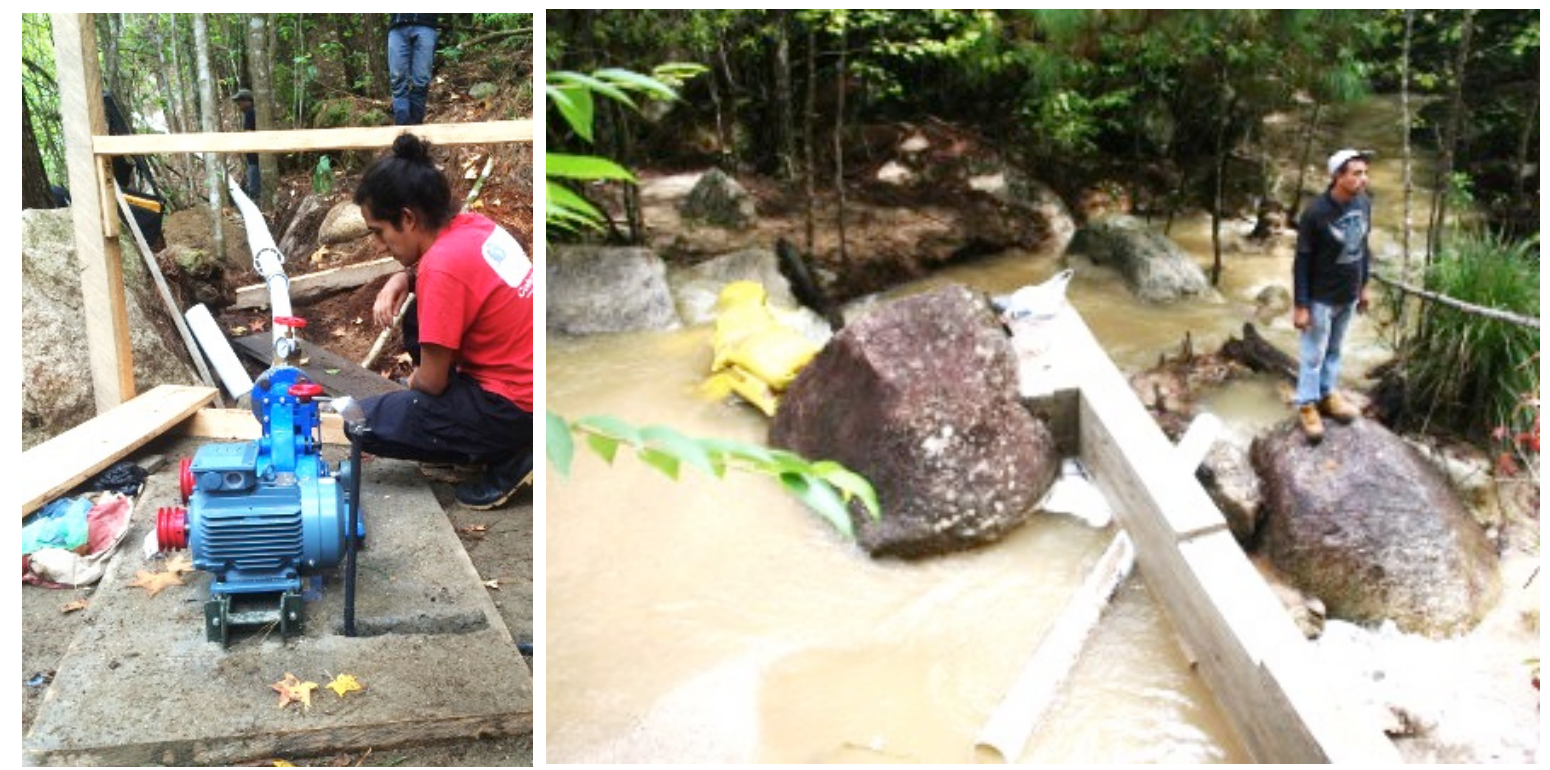

[6.15] Turbine and Generator connected to Penstock (Rodriguez, 2015)

[6.16] Damn and Intake (author's photo)

Most sediment and organic waste that enter the turbine are flushed away automatically. Due to its self-cleaning design and standardized componentry, the turbine maintenance is very low.. "and should operate efficiently for at least 40 years" (CEO, n.d.: 41). It is important to keep bearings lubricated and to clean the turbine from time to time. Apart from that, no maintenance on the turbine itself is necessary. 


\subsubsection{Pumps as Turbines}

The principal research on centrifugal pumps was conducted by the German engineer Carl Pfleiderer. He published much of his work in the first half of the 19th Century, holds many national and international patents, and might be seen as the "father" of the theoretical background of centrifugal pumps. A typical Francis

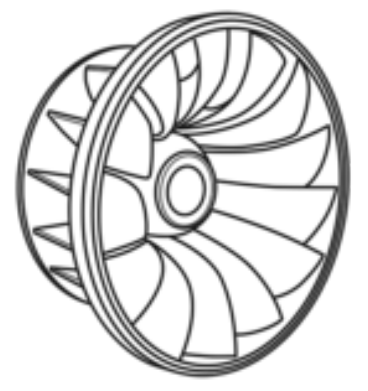
runner is shown on the right side (Vöstalpine, n.d.). In the second half of the 19th Century, research was conducted to predict the behavior of "pumps as turbines" (PAT). In the 1980s, lot of PAT research was done by the English engineer Arthur Williams.

The runner of a centrifugal pump is of the same design as the runner of the Francis turbine: "Francis turbines are radial flow reaction turbines, with fixed runner blades and adjustable guide vanes, used for medium heads" (ESHA, 1998: 159). Francis turbines are mainly used for large hydro sites. In these large hydropower stations, the Francis turbine is fitted with guide vanes. Guide vanes "regulate the water flow as it enters the runner, and usually are linked to a governor system, which matches flow to turbine loading” (Harvey, 1993: 178). As we see in the case of the Kaplan turbine, the same regulation system is used for Francis turbines. But this sophisticated regulation system is too complex and too costly for micro and pico hydropower systems, and therefore, there are no adjustable guide vanes available.

Usually, a pump pushes water to a higher potential energy level with the help of electromechanical energy. A turbine does the opposite. A turbine uses the potential energy of water and transforms it into mechanical energy. Under certain conditions, a reverse turning pump could be used as a turbine. The basic hydraulic theory is the same for both machines. The sketch 6.17 depicts the two working principles, followed by a table illustrating the 
differences between a pump and a turbine. As we can see, the operating conditions of a pump and a turbine are different. A pump usually operates with constant speed, head and flow, and is "therefore designed for one particular point (duty point) of operation and does not require a regulating device (guide vane)" (Chapallaz et al. 1992: 9). Turbines, on the other hand, operate under variable head and flow conditions.
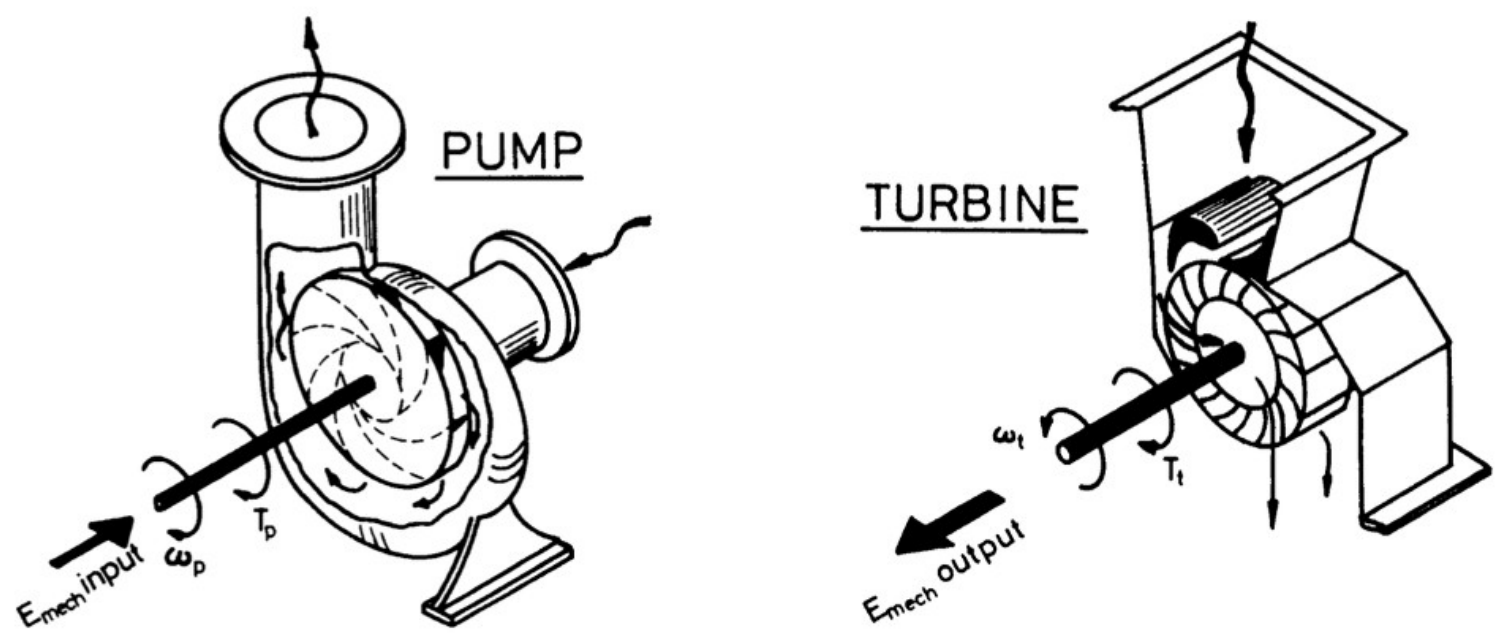

\begin{tabular}{|l|l|l|}
\cline { 2 - 3 } \multicolumn{1}{l|}{} & Turbine & Pump \\
\hline \hline Flow of energy & $\begin{array}{l}\text { Input of hydraulic energy (water } \\
\text { under pressure) } \\
\text { Output of mechanical energy } \\
\text { (torque on the shaft) }\end{array}$ & $\begin{array}{l}\text { Input of mechanical energy (torque } \\
\text { on the shaft) } \\
\text { Output of hydraulic energy (water } \\
\text { under pressure) }\end{array}$ \\
\hline $\begin{array}{l}\text { Hydraulic } \\
\text { pressure head }\end{array}$ & $\begin{array}{l}\text { Available turbine head decreases } \\
\text { with increasing flow (friction } \\
\text { losses) }\end{array}$ & $\begin{array}{l}\text { Total dynamic head to be } \\
\text { generated by the pump increases } \\
\text { with increasing flow. }\end{array}$ \\
\hline $\begin{array}{l}\text { Direction of } \\
\text { rotation }\end{array}$ & Turbine runner rotates in the opposite direction of a pump impeller. \\
\hline $\begin{array}{l}\text { Direction of } \\
\text { torque }\end{array}$ & In both operation modes the direction is the same. \\
\hline
\end{tabular}

[6.17] Sketch and Comparison Table of working Principle of Pumps and Turbines (Chapallaz, Eichenberger \& Fischer, 1992: 9)

The following photos 6.18 show a Francis turbine with regulated guide vanes (on left) and two PATs (on right). The PATs work without moveable guide vanes, with fixed head and flow, and directly coupled to the generators. 

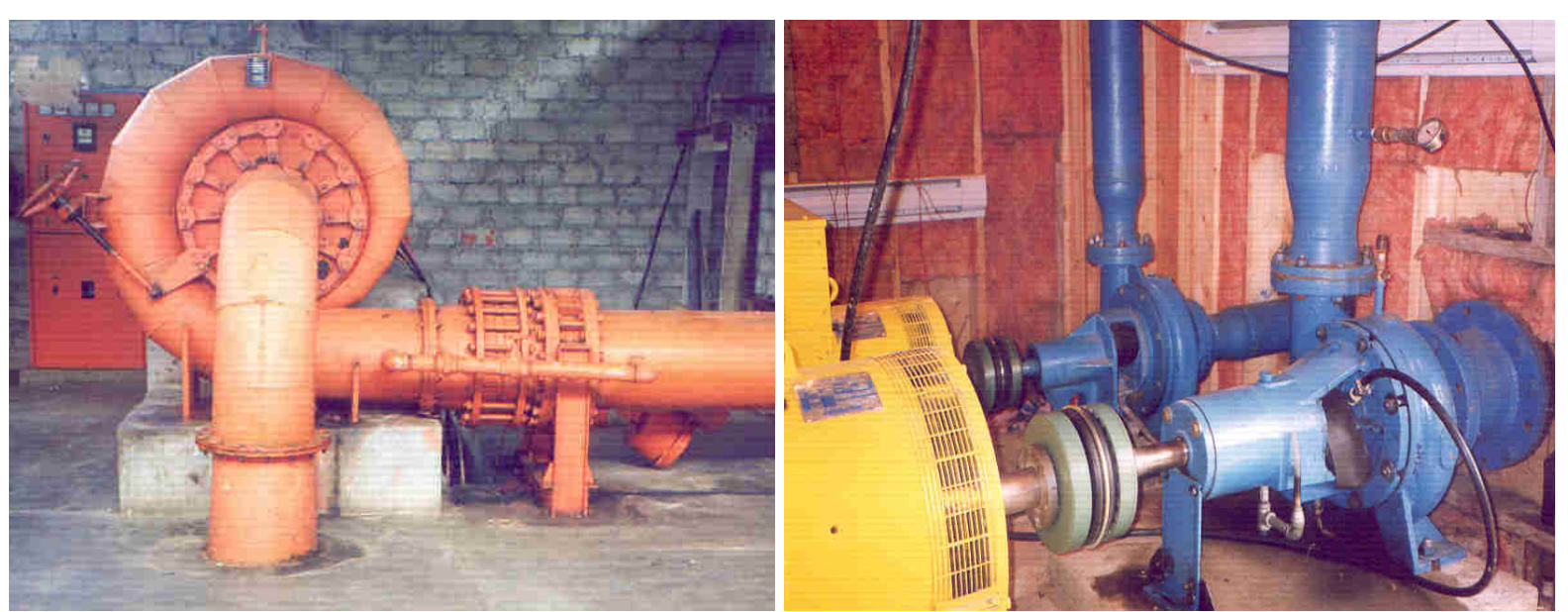

[6.18] Francis Turbine (left) and PAT directly connected to Generator (right) (Dávila, 2010)

The hydraulic design of a Francis turbine and a centrifugal pump is identical: "When operating a pump as a turbine, the flow pattern through the machine is similar to the flow conditions in a turbine" (Chapallaz et al. 1992: 9). The way of the water flow is described as follows with the words of Ray Holland: "The water enters from around the periphery of the runner (normally via a spiral casing), passes through the guide-vanes and runner blades and exits axially from the center" (Holland, 1983: 14). In operation, the water proceeds through the turbine as if it were enclosed in a closed-conduit pipe. It moves from "a fixed component, the distributor, to a moving one, the runner, without being at any time in contact with the atmosphere" (ESHA, 1998: 160). In turbine operation, the water enters radial and leaves the runner in axial direction. The runner receives most of the waters pressure energy, whereas the water leaves the turbine through the draft tube (Harvey, 1993: 178). What dictates the use of a pump as a turbine is the flow rate. The flow rate cannot be adjusted, and therefore, PAT systems are only suitable for sites where there is sufficient water supply throughout the year.

\section{Technical Complexity and Efficiency:}

Standard centrifugal pumps are manufactured in a number of sizes, to cover a wide range of heads and flows. Given the right conditions, pumps as turbines can be used over the range 
normally covered by multi-jet Pelton turbines and Cross Flow turbines. The use of a pump as a turbine has the greatest advantage in terms of cost and simplicity. Pumps are manufactured in many countries, mainly for water supply and irrigation purposes. Often, there are regional manufacturers of pumps but not of turbines in these countries. Therefore, PATs may be more economical, since shipping costs and import taxes are usually less

A purpose-built water turbine is generally fitted with a variable guide vane or a spear valve, which allows the machine to run efficiently with a wide range of flow rates. When a standard centrifugal pump is used as a turbine, no such adjustment is possible. However, once installed, a PAT that is well-matched to the site conditions will operate close to its maximum efficiency: "The efficiency of the pump turbine falls off rapidly as the flow rate reduces, and at $50 \%$ flow no power is delivered at all, but provided the water flow can be guaranteed when the power is needed, the saving in capital cost may be worthwhile” (Holland, 183: 14). Obviously, a huge disadvantage of PATs is the limitation of their flow rate. As we can see in the efficiency characteristics in sketch 6.20 , the performance of a PAT system is limited to a very small flow range. The part flow efficiency is very low, and therefore, PATs are not applicable for many sites.
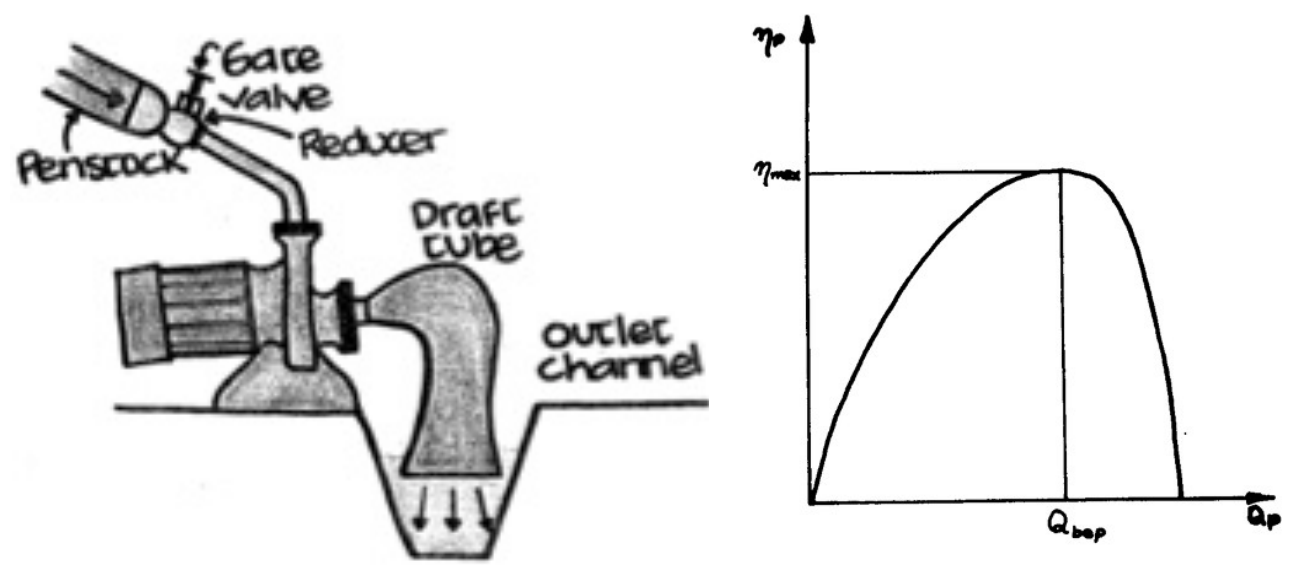

[6.19] PAT Principle Sketch (Hernandez, 2012)

[6.20] PAT Efficiency Curve, (Williams, 1995: 16) 
However, the very low part flow efficiency problem can be solved by using two or more units of different size, and switching between them depending on the flow rate. But having several machines leads to higher costs.

Advantages of PATs:

- Integral pump and motor can be purchased for use as a turbine and generator set

- Available for a wide range of heads and flows

- Available in a large number of standard sizes

- Short delivery time

- Spare parts such as seals and bearings are easily available

- Easy installation - uses standard pipe fittings

- Direct drive, no belt drive needed

- Very low friction loss in drive

- Easier installation, PAT and generator come as one unit

- Lower cost - no pulleys, smaller base plate

- Lower cost (mono-bloc design, simpler construction, fewer bearings, etc.)

○ Less maintenance

\section{Fabrication:}

There is no need to locally manufacture pumps as turbines. PATs are a standard product, mass fabricated and available at decent prices all over the world: "Standard pumps are simple and do not require highly qualified mechanics for maintenance; this makes standard pumps as turbines more appropriate for developing countries than sophisticated turbines" (Chapallaz et al., 1992: 4).

\section{Installation and Maintenance:}

The installation of a Cross Flow turbine is exactly the same as for a PAT, Turgo or Pelton turbine. An intake is necessary, mostly consisting of a small dam and some sediment protection according to the site's water condition. The scheme then consists of a penstock, the turbine house with its electromechanical equipment, and the transmission and distribution 
lines. The only difference is the turbine itself, that instead of a Pelton or Cross Flow, a PAT is used as the turbine.

The following photograph 6.21 depicts a PAT installation in San Antonio, Honduras followed by a PAT system with a separate coupled generator 6.22 .
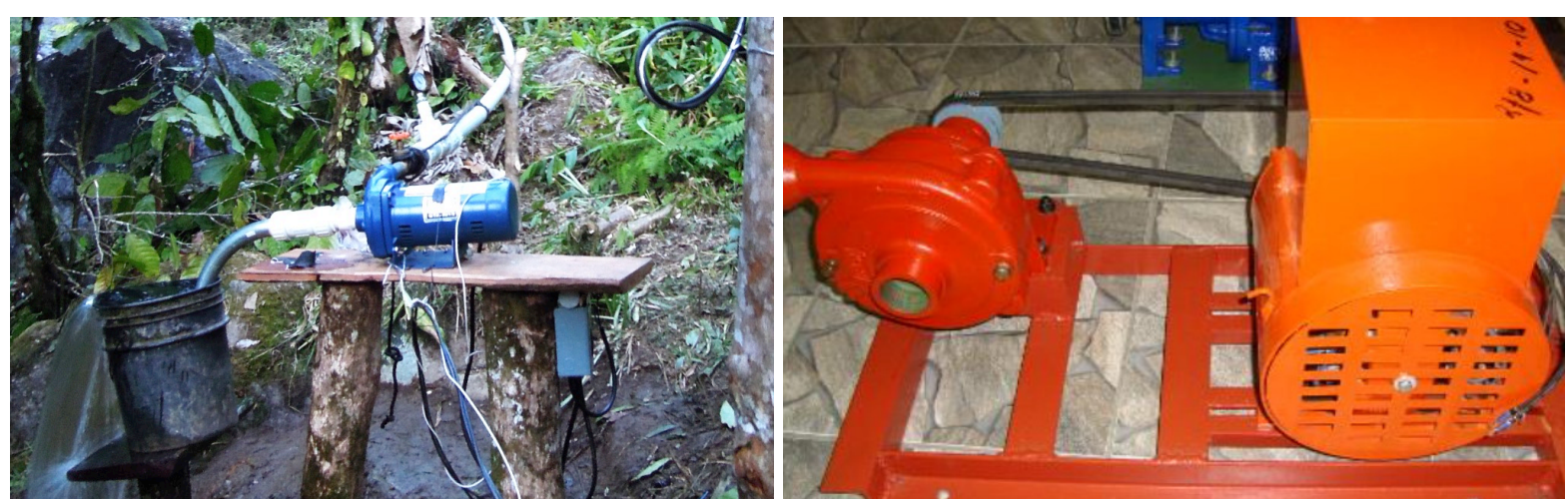

[6.21 / 6.22] PAT Installation in Honduras and PAT with separate Generator, (author's photo, 2012)

The maintenance of a pump as turbine depends strongly on its size and on the water conditions. The smaller the turbine, which is the case in pico hydropower, the more difficulties arise through sediment and organic waste, which are almost impossible to keep outside of the penstock. The very small Francis runner used in these turbines consists of a tiny entrance area for the incoming water. In my experience with installations in Honduras and Nicaragua, I have seen sediment blocking these spaces and hindering the water from entering the turbine.

Depending on the design of the entrance of the penstock and the amount of sediment in the water, this happens several times a year. When blocked, the turbine has to be opened and cleaned. However, the cleaning itself is not a time-consuming job since just a few screws from the PAT case have to be opened to access the runner. Apart from that, the typical maintenance of bearings is necessary, as with other turbines, as well. 


\subsubsection{The Turgo Turbine}

Next on the list from low head to high head turbines covered in this study, the following chapter covers the Turgo turbine. The Turgo turbine is an impulse-type water turbine design based on the Pelton turbine. Most of the characteristics of Pelton turbines are similar and also valid for Turgo turbines. Since the Pelton turbine is discussed in detail in the next chapter, this chapter only covers briefly the differences of the Pelton turbine. To understand the working principle of the Turgo turbine, it is necessary to read the chapter about the Pelton, as well, perhaps before reading this chapter.

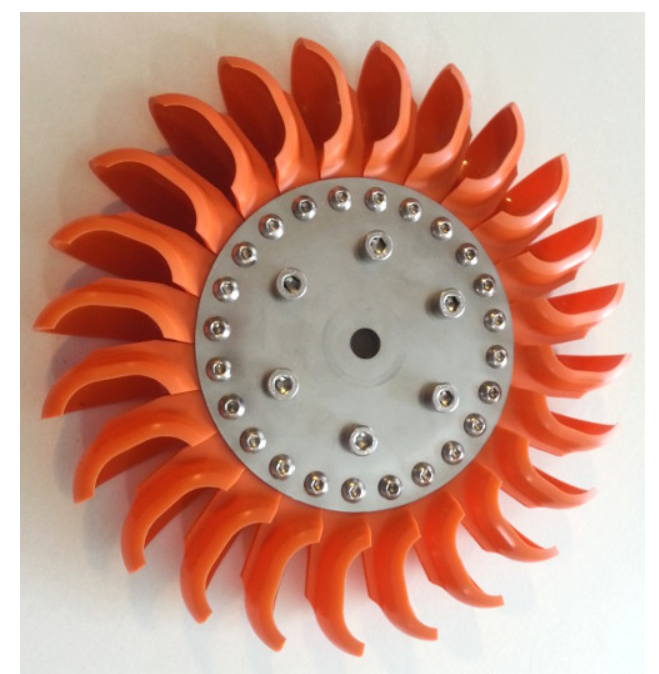

[6.23] Turgo Runner (Hartvigsen, n.d.)

For easier understanding, a typical Turgo runner with nylon spoons is illustrated above. Turgo turbines, like Pelton turbines, are typically used for lower heads and higher flow. The Turgo turbine is an impulse turbine "but its buckets are shaped differently and the jet of water strikes the plane of its runner at an angle of 20" (ESHA, 1998: 158) instead of tangentially. It is designed so that water enters at one side and exits at the other, as seen in illustration 6.24. In this way, the jet of incoming water is not hindered by the jet of outgoing water. The curved blades catch the water as it flows through the turbine and cause the turbine shaft to rotate. "As a consequence of this, the flow that a Turgo runner can accept is not 
limited by spent fluid interfering with the incoming jet (as it is the case with Pelton turbine).

Hence, a Turgo turbine can have a smaller diameter runner than a Pelton for an equivalent power. It therefore runs at a higher speed" (Harvey, 1993: 172).
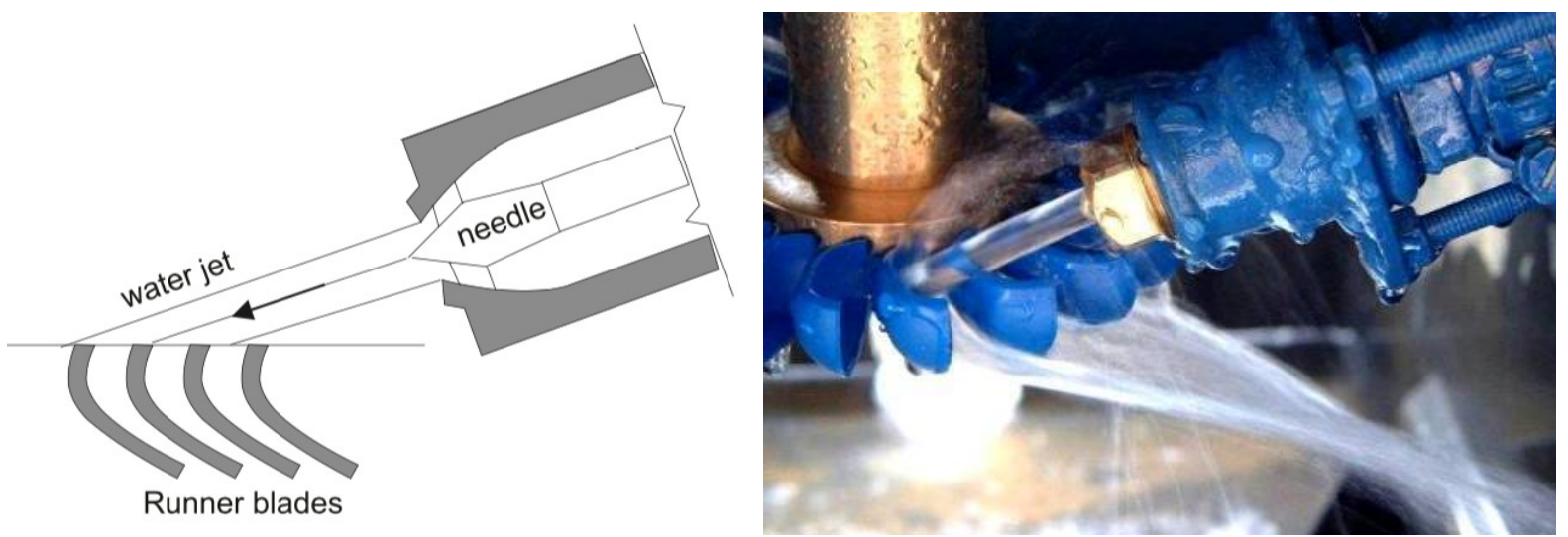

[6.24] Water Flow through a Turgo Turbine Runner (ESHA, 1998: 158)

[6.25] Water Jet impinging on the Rotor of a Turgo Runner (Renewables First, 2015)

This is a significant advantage, as "the resulting higher runner speed of the Turgo makes direct coupling of turbine and generator more likely (ESHA, 1998: 158). A direct-coupled generator will increase the overall efficiency because there is no need for a belt, and therefore no losses caused by friction. Like the Pelton turbine, the Turgo is efficient over a wide range of speeds. However, the Turgo runner is slightly less efficient than the Pelton runner and has a certain disadvantage regarding its design: "The Turgo experiences a substantial axial load on its runner which must be met by providing bearing on the end of the shaft" (Harvey, 1993:

172), which can be dealt with by appropriate shaft and bearing design. The following pictures 6.26 show a compact Turgo turbine design from China.
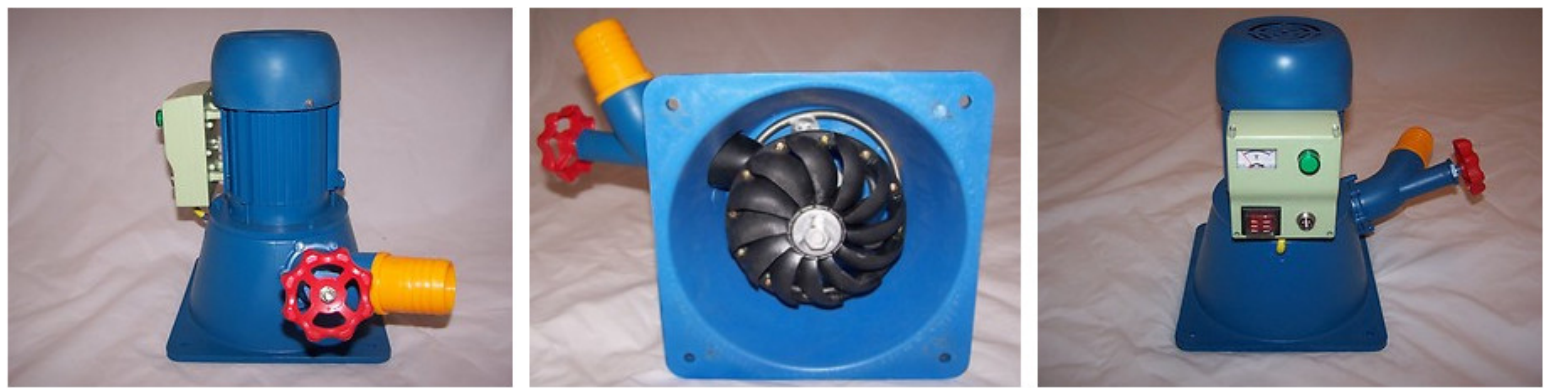

[6.26] A Chinese Design of a 300W Pico Turgo Turbine (Micro Hydro Power, 2015) 
In recent years, manufacturers of Turgo spoons appeared on the market, which is interesting from a DIY perspective. The manufacturers produce spoons in different sizes and materials (either in nylon or stainless steel). The spoons can be assembled into a rotor with a diameter to suit the head at the site. A typical DIY Turgo turbine rotor with nylon spoons is shown in figure 6.23 . These spoons are very small and can be easily shipped all over the world without significant costs. This is a very recent development and might affect the pico hydropower market in the near future.

\subsubsection{The Pelton Turbine}

Pelton turbines are a type of high head impulse turbine that "evolved during the gold rush days of the late nineteenth century California, [and] was patented by Lester Pelton in 1880" (Boyle, 2004: 169). The Pelton consists of a movable wheel with buckets on its periphery, over which one or several water jets impinge at atmospheric pressure. The jets flow through several nozzles, which control the flow through a needle valve (ESHA, 1998: 157). The runner is provided with identical buckets, ranging between 18 and 28, and is of robust design with strong durability. The important power transforming elements of this turbine are the buckets and the nozzle. Basically, it is a wheel with a set of double cups or buckets attached around the rim as depicted in sketch 6.27. The water hits the runner tangentially through one or more jets. Each jet issues through a nozzle with a needle valve to control the flow. The nozzle forms and directs the jet against the bucket. The jet of water "hits the splitting edge between each pair of cups in turn as the wheel spins. The water passes round the curved bowls, and under the optimum conditions gives up almost all kinetic energy" (Boyle, 2004: 169). The water exiting the vanes slide at atmospheric pressure through the walls of the housing and return to the stream or river. 

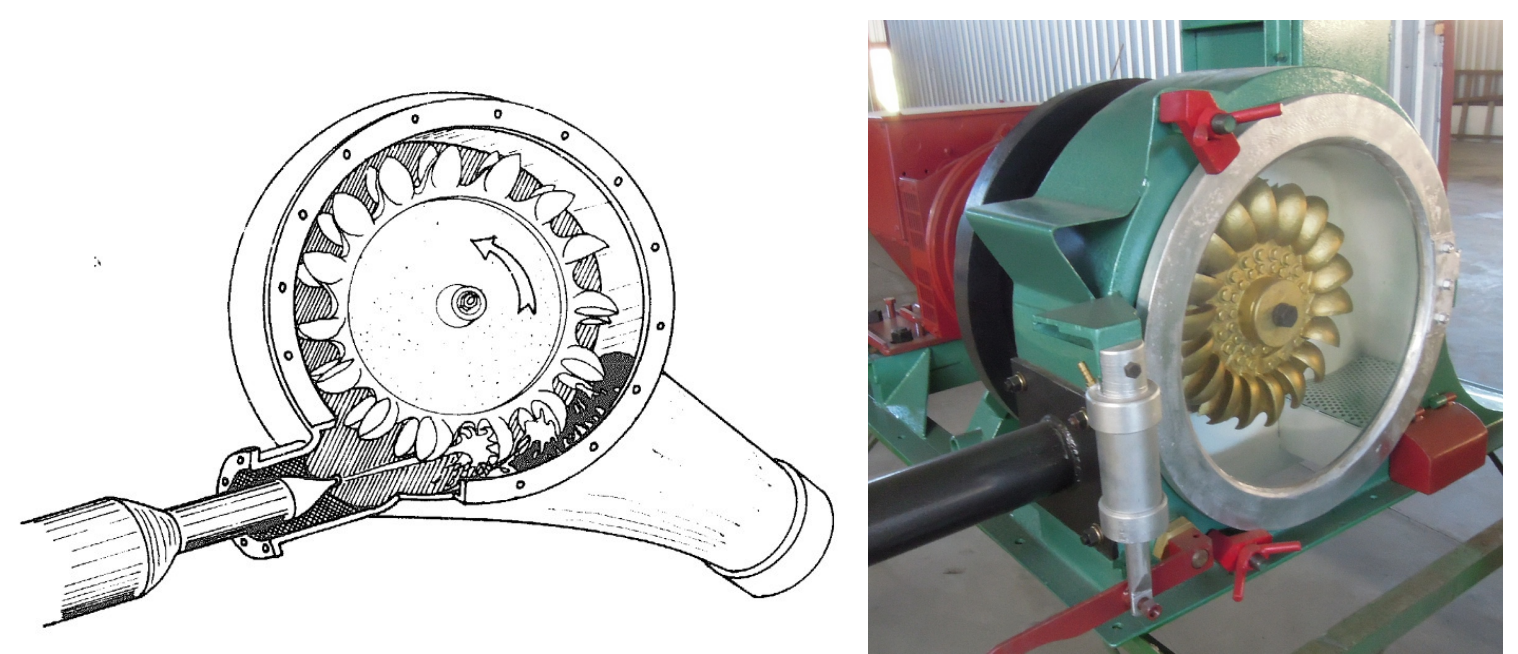

[6.27] Pelton Turbine Working Principle (Alward, Eisenbart \& Volkman, n.d.: 21)

[6.28] Pelton Turbine fabricated in Nicaragua (author's photo, 2012)

The function of the bucket is to convert the jet, represented by its high speed, into mechanical energy at the wheel shaft. Therefore, the buckets are designed to keep the exit velocities of the water to a minimum: "To do this, it must slow down the water from its high speed in the jet to practically zero speed when it drops into the tail water" (MacKillop, 1983: 35). This can be best achieved if the buckets are designed in the size and form presented in photo 6.29 and sketch 6.30 .
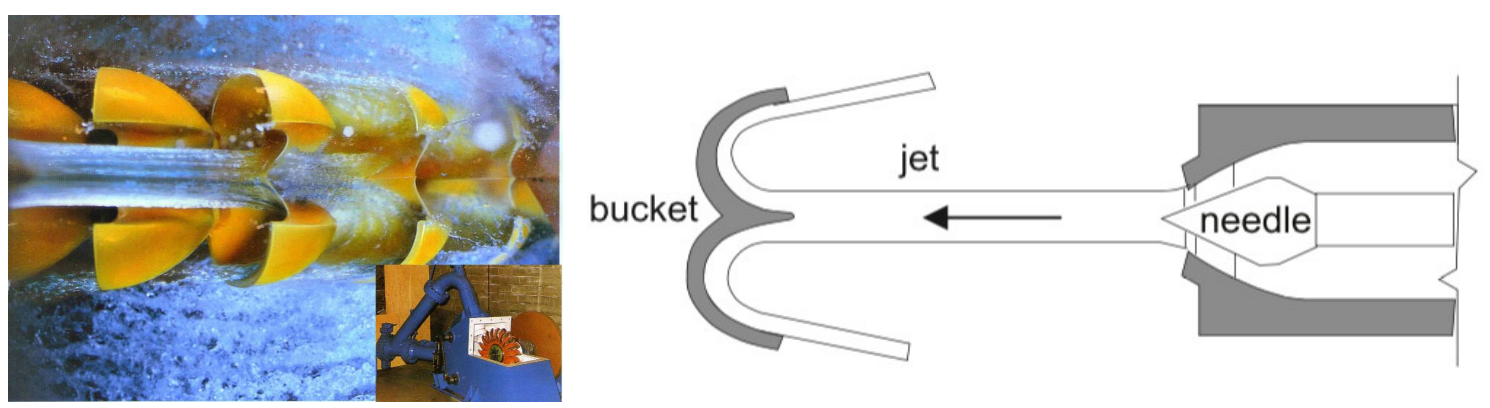

[6.29] Flow of water in a Pelton runner (Dávila, 2010)

[6.30] Pelton water flow theory (ESHA, 1998:157)

This shape serves to slow the jet by turning it smoothly through almost 180 degrees. In order to do so, the surface of each bucket is important and must be as smooth as possible, and all buckets must be as uniform as possible. Another important concern is the correct orientation of the jet to the bucket, since the jet must impinge upon each bucket at the correct wheel 
radius or lever arm. When the full jet strikes, in theory, the bucket should be perpendicular to reach highest efficiency. Practically, this is not possible, since the water has to exit.

The distributor of large Pelton turbines could have up to six injector jets (vertical axis design) of water impinging on the runner at the same time. For micro and pico hydro application systems, up to four jets are available.

To stop the turbine, the jet of water may be deflected by a plate so that it does not hit the buckets. This may be necessary due to load rejection as the turbine accelerates and reaches runaway speed.

\section{Technical Complexity and Efficiency:}

Since the Pelton design has been in use for many years, there is a lot of shared experience and knowledge available on how to design such a turbine with high efficiency. Boyle (2004: 170 explains): "The efficiency of a Pelton wheel is greatest when the speed of the cups is half the speed of the water jet. As the cup speed depends on the rate of rotation and the wheel diameter, and the water speed depends on the head, there is an optimum relationship between these three factors" (Boyle, 2004: 170).

Nevertheless, it is very important to have a turbine designed to work in the specific conditions at the selected site. As Boyle states: "If adjacent cups are not to interfere with the flow, the wheel diameter needs to be about ten times the diameter of the jet. But two or even four jets can be spaced around the wheel to give greater output without increasing the size" (2004: 170). It is very visible, that the hydraulic design of a hydro turbine is a complex task and many design rules have to be applied. 
Experiences show that small turbine manufacturers lack adequate knowledge of turbine design. Therefore, turbines might not have the right diameter, may not reach the adequate speed for the generator, etc. A minor example of these "mistakes" is shown in the following picture 6.31 depicting a Pelton turbine fabricated and installed in northern Honduras.

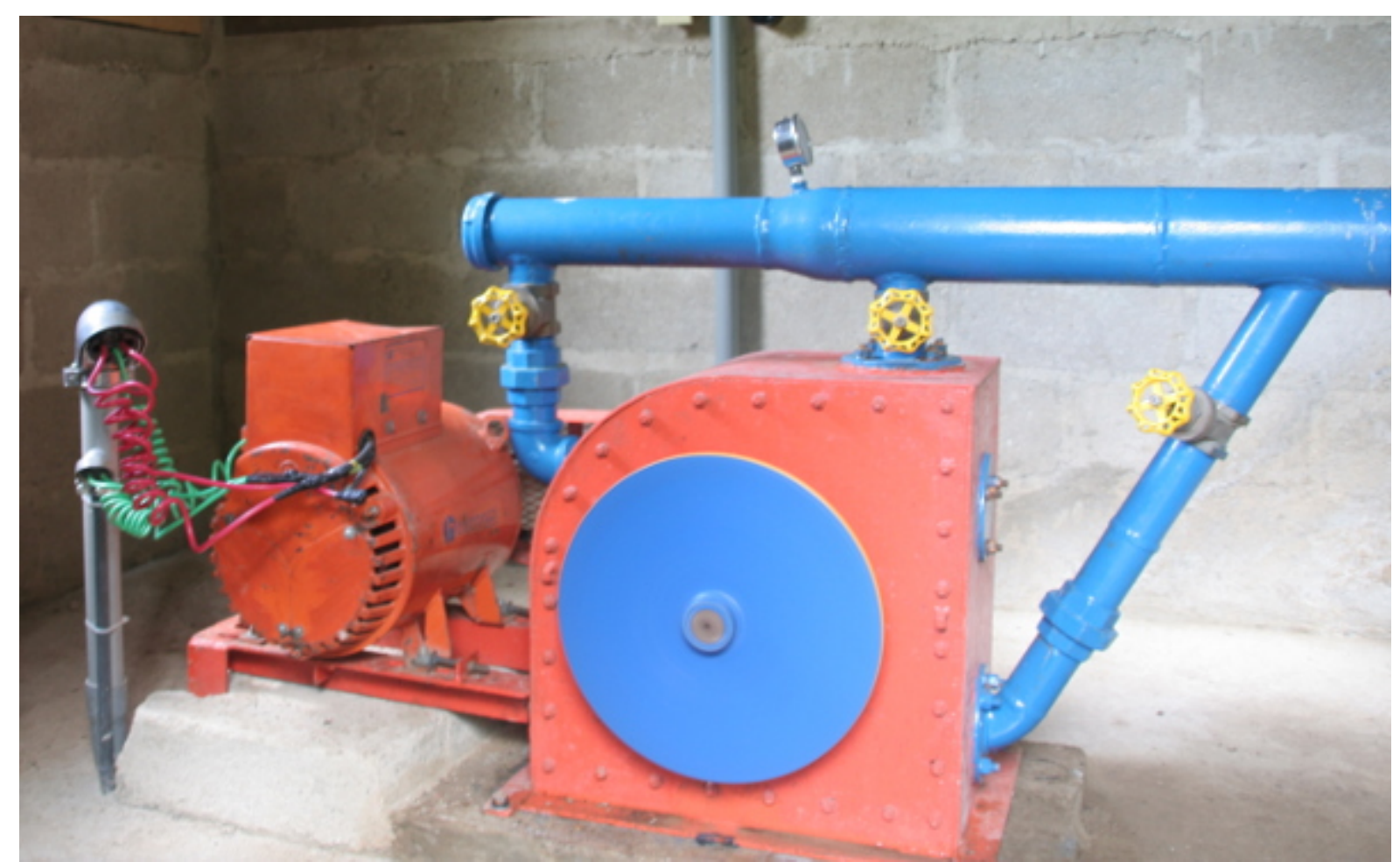

[6.31] Three Jet Pelton Turbine with Flywheel, fabricated and installed in Honduras (author's photo, 2013)

This is a three jet Pelton turbine, and in the distributor piping we see a 90 degree angle twice, which is a harsh direction change, provoking turbulences resulting in energy losses. These angles should be more smooth, as the first jet is designed. These small mistakes, taken together, produce a remarkable loss of efficiency of the overall system.

\section{Fabrication:}

The most important aspect of manufacturing is the shape of the buckets. Even assuming that a highly efficient bucket design could be calculated or available for free, a crucial problem remains. The manufacturing of the buckets is rather difficult, especially for a DIY solution. In 
general, these buckets are made out of forged steel. Casted buckets are also available, but involve a more complex form of manufacturing that requires appropriate machines and adequate experience.

Other parts of the turbine are comparably easy to manufacture. The shaft and the casing do not require a sophisticated design: "The turbine casing only needs to protect the surroundings against water splashing and therefore can be very light" (ESHA, 1998: 157).

\section{Installation and Maintenance:}

The installation of a Cross Flow turbine is exactly the same as for a PAT, Turgo or Pelton turbine. An intake is necessary, mostly consisting of a small dam and some sediment protection according to the site's water condition. The scheme then consists of a penstock, the turbine house with its electromechanical equipment, and the transmission and distribution lines. Pelton turbines are high head turbines. Distances tend to be longer, and there are height differences, as well. Therefore, a proper penstock design becomes more important, or even crucial. Apart from that, the only difference is the turbine itself, that instead of a PAT or Cross Flow, a Pelton is used as the turbine.

The Pelton turbine runs rather maintenance-free and can be compared with the Cross Flow and Turgo turbine schemes. The intake has to be cleaned from time to time, depending on the sediment density of the site. On the other side, the injector(s) has to be cleaned, if necessary. Most of the time, sediments are flushed away by the high speed of water leaving the injector nozzle. 


\subsection{Comparison of Turbine Types}

It is important to note that each site has different characteristics and requires a system designed to its specific and unique conditions. Due to the effort and expense of designing a hydroelectric system, it is recommended to use a flexible flow turbine, which has a wide application range. In this way, the design costs can be minimized. The advantages and disadvantages of the different turbine types shown above are summarized and illustrated in the following table 6.32 . 
Feasibility of an Open Source Hydro Turbine

\begin{tabular}{|c|c|c|c|c|c|}
\hline & Pelton & Turgo & $\begin{array}{l}\text { Michell Banki / } \\
\text { Cross Flow }\end{array}$ & $\begin{array}{l}\text { Pump as Turbine } \\
\text { PAT (Francis) }\end{array}$ & Kaplan / Axial \\
\hline $\begin{array}{l}\text { Application range } \\
\text { (Pico) }\end{array}$ & $\begin{array}{l}30-80[\mathrm{~m}] \\
3-12[\mathrm{l} / \mathrm{s}]\end{array}$ & $\begin{array}{l}25-60[\mathrm{~m}] \\
4-12[\mathrm{l} / \mathrm{s}]\end{array}$ & $\begin{array}{l}8-40[\mathrm{~m}] \\
12-60[\mathrm{l} / \mathrm{s}]\end{array}$ & $\begin{array}{l}25-50[\mathrm{~m}] \\
5-10[\mathrm{l} / \mathrm{s}]\end{array}$ & $\begin{array}{l}1.5-3[\mathrm{~m}] \\
30-120[\mathrm{l} / \mathrm{s}]\end{array}$ \\
\hline $\begin{array}{l}\text { Availability of } \\
\text { technology in the } \\
\text { market }\end{array}$ & $\begin{array}{l}\text { Yes, but limited to a few } \\
\text { manufacturers and } \\
\text { therefore has to be } \\
\text { shipped and imported }\end{array}$ & $\begin{array}{l}\text { Yes, but limited to a few } \\
\text { manufacturers and } \\
\text { therefore has to be } \\
\text { shipped and imported }\end{array}$ & $\begin{array}{l}\text { Yes, but limited to a few } \\
\text { manufacturers and } \\
\text { therefore has to be } \\
\text { shipped and imported }\end{array}$ & $\begin{array}{l}\text { Yes, a wide range of } \\
\text { pumps from various } \\
\text { manufacturers are } \\
\text { available in almost every } \\
\text { country }\end{array}$ & $\begin{array}{l}\text { Yes, but limited to a } \\
\text { few manufacturers in } \\
\text { Asia }\end{array}$ \\
\hline $\begin{array}{l}\text { Fabrication } \\
\text { Complexity }\end{array}$ & $\begin{array}{l}\text { Low, but needs casting } \\
\text { for runner fabrication }\end{array}$ & $\begin{array}{l}\text { Low, but needs casting } \\
\text { for runner fabrication }\end{array}$ & $\begin{array}{l}\text { Very Low, welding } \\
\text { design }\end{array}$ & $\begin{array}{l}\text { Complex, but available in } \\
\text { almost every country for } \\
\text { little cost }\end{array}$ & $\begin{array}{l}\text { Very Low, welding } \\
\text { design }\end{array}$ \\
\hline $\begin{array}{l}\text { Overall project } \\
\text { Costs (tendency, each } \\
\text { project is } \\
\text { different, please } \\
\text { chapter see } \\
\text { Economic Aspects for } \\
\text { further information) }\end{array}$ & $800-4500$ [US \$ / kW] & $800-4500$ [US \$ / kW] & $800-4500$ [US \$ / kW] & $500-2500[\mathrm{US} \$$ / kW] & $\begin{array}{l}2500-6000 \text { [US \$ / } \\
\mathrm{kW}]\end{array}$ \\
\hline Turbine Costs & $\begin{array}{l}\text { Expensive because of } \\
\text { shipping and customs }\end{array}$ & $\begin{array}{l}\text { Expensive because of } \\
\text { shipping and customs }\end{array}$ & $\begin{array}{l}\text { Expensive because of } \\
\text { shipping and customs }\end{array}$ & $\begin{array}{l}\text { Affordable, cheaper as } \\
\text { turbines }\end{array}$ & $\begin{array}{l}\text { Affordable through } \\
\text { local fabrication }\end{array}$ \\
\hline $\begin{array}{l}\text { Overall project } \\
\text { setting }\end{array}$ & $\begin{array}{l}\text { Because of high } \\
\text { pressure, mostly long } \\
\text { penstock is needed, but } \\
\text { very little civil works }\end{array}$ & $\begin{array}{l}\text { Because of high } \\
\text { pressure, mostly long } \\
\text { penstock is needed, but } \\
\text { very little civil works }\end{array}$ & $\begin{array}{l}\text { Little civil works are } \\
\text { needed and penstock at } \\
\text { medium pressure. But } \\
\text { penstock with big } \\
\text { diameter is needed } \\
\text { because of high flow. }\end{array}$ & $\begin{array}{l}\text { Little civil works are } \\
\text { needed and penstock at } \\
\text { medium pressure and } \\
\text { low flow rate. }\end{array}$ & $\begin{array}{l}\text { Because of high flow, } \\
\text { complex civil works } \\
\text { are necessary and } \\
\text { expensive. }\end{array}$ \\
\hline $\begin{array}{l}\text { Efficiency and part } \\
\text { flow efficiency }\end{array}$ & Good efficiency & $\begin{array}{l}\text { Good efficiency, almost } \\
\text { as good as Pelton } \\
\text { turbine. }\end{array}$ & $\begin{array}{l}\text { Efficiency is not very } \\
\text { high, but remains stable } \\
\text { over a wide range of } \\
\text { flow. }\end{array}$ & $\begin{array}{l}\text { Acceptable efficiency but } \\
\text { highly depends on the } \\
\text { model. Part flow } \\
\text { efficiency is very low. } \\
\text { PAT therefore needs a } \\
\text { fixed flow rate. }\end{array}$ & $\begin{array}{l}\text { The "Simplex" } \\
\text { prototype fabricated in } \\
\text { Nicaragua had a very } \\
\text { low efficiency. Pico } \\
\text { Kaplan turbines are } \\
\text { not regulated and } \\
\text { therefore count as }\end{array}$ \\
\hline
\end{tabular}


Feasibility of an Open Source Hydro Turbine

\begin{tabular}{|c|c|c|c|c|c|}
\hline & & & & & $\begin{array}{l}\text { axial turbines. Axial } \\
\text { turbines have a very } \\
\text { poor part flow } \\
\text { efficiency. }\end{array}$ \\
\hline Maintenance & low & low & low & medium & high \\
\hline $\begin{array}{l}\text { Technical } \\
\text { Complexity }\end{array}$ & low & low & low & $\begin{array}{l}\text { Low and high: The } \\
\text { technical complexity of } \\
\text { the turbine is low. But } \\
\text { the "translation" of the } \\
\text { application range from } \\
\text { "pump" to "turbine" } \\
\text { needs calculation and } \\
\text { minor adaptions, which } \\
\text { are rather complex. }\end{array}$ & high \\
\hline Market / Demand & $\begin{array}{l}\text { High, but limited } \\
\text { through its application } \\
\text { range (height). }\end{array}$ & $\begin{array}{l}\text { High, but limited } \\
\text { through its application } \\
\text { range (height). }\end{array}$ & $\begin{array}{l}\text { Very high, because of its } \\
\text { broad application range } \\
\text { (head and flow). }\end{array}$ & $\begin{array}{l}\text { High, but limited } \\
\text { through its application } \\
\text { range (height) and very } \\
\text { low part flow efficiency. }\end{array}$ & $\begin{array}{l}\text { Rather low, if no } \\
\text { already installed dam } \\
\text { could be used, civil } \\
\text { works costs are very } \\
\text { high. }\end{array}$ \\
\hline Observations & $\begin{array}{l}\text { - Availability limited } \\
\text { - Spare parts not } \\
\text { available } \\
\text { - Good efficiency } \\
\text { - Simple working } \\
\text { principle }\end{array}$ & $\begin{array}{l}\text { - Availability limited } \\
\text { - Spare parts not } \\
\text { available } \\
\text { - Good efficiency } \\
\text { - Simple working } \\
\text { principle }\end{array}$ & $\begin{array}{l}\text { - local fabrication } \\
\text { - spare parts availability } \\
\text { - low cost } \\
\text { - flexibility } \\
\text { - low maintenance } \\
\text { - less peak efficiency } \\
\text { than other turbines }\end{array}$ & $\begin{array}{l}\text { - each model has to be } \\
\text { tested, since the } \\
\text { performance as turbine } \\
\text { is just theory, surprises? } \\
\text { - low cost } \\
\text { - good overall efficiency } \\
\text { - very low part flow } \\
\text { efficiency }\end{array}$ & $\begin{array}{l}\text { - easy design } \\
\text { - robust } \\
\text { - low efficiency } \\
\text { - the "Simplex" design } \\
\text { is not market-ready } \\
\text { - complex and costly } \\
\text { civil works }\end{array}$ \\
\hline
\end{tabular}

[6.32] Comparison of different hydro Turbines (author's illustration) 
The site itself, with its specific head and flow, distances and terrain, defines the appropriate technical solution. For covering these options, several turbine types have been developed. Looking at the advantages and disadvantages in the selected categories, it seems that the Cross Flow turbine is the most appropriate design for an open source DIY application. The Cross Flow turbine offers a broad application range, comparably low manufacturing complexity at decent costs, and low maintenance. The Banki turbine seems to have a lot of advantages for rural electrification and also as a DIY turbine design.

In conclusion, the Cross Flow design carries the greatest advantage for a locally replicable hydropower turbine. The following chapters explore the Cross Flow turbine from different perspectives.

\subsection{Economic Aspects}

It is very difficult to get specific figures about project costs, and they differ widely around the globe depending on the local circumstances, prices and tax. In the power range of up to $5 \mathrm{~kW}$, "three out of four machines are PAT systems; one is a banki turbine" (Gnecco, 2012). Standardized products like pumps and motors are much cheaper than a "water turbine" of this size. Roughly, the calculation of PAT systems in Colombia is around \$1200 USD for $1 \mathrm{~kW}$ (Gnecco, 2012). The following table depicts the cost-estimations of different turbine types in Colombia, divided into percentages based on where the money is allocated:

\begin{tabular}{|l|l|l|}
\hline Type of Turbine & Distribution of Costs & Costs [US \$ / kW] \\
\hline Axial Turbines & CW: $50 \%$ & $2500-6000$ \\
& T: $30 \%$ & \\
& EME: $10 \%$ & \\
\hline
\end{tabular}

$-203-$ 


\begin{tabular}{|l|l|l|}
\hline & E: $10 \%$ & \\
\hline & CW: $35 \%$ & $500-2500$ \\
& T: $20 \%$ & \\
& EME: $25 \%$ & \\
& E: $20 \%$ & $800-4500$ \\
& CW: $30 \%$ & \\
Impulse Turbines & T: $30 \%$ & \\
(Pelton, Banki) & EME: $30 \%$ & \\
& E: $10 \%$ & EME: Electromechanical Equipment \\
CW: Civil Works & \multicolumn{2}{|l|}{} \\
T: Turbine & E: Extras \\
\hline
\end{tabular}

[6.33] Comparison of HydroPower Costs (Gnecco, 2012)

The distribution of costs seems realistic compared to the experience in Nicaragua. However, the cost per kilowatt is definitely higher in Nicaragua. All of this depends highly on the price of materials, import taxes, manufacturing and labor costs in different countries. And, importantly, it also depends on the site itself, since environments and conditions vary from site to site. The same site conditions (height and flow) should result in the same project costs. In reality project costs could double from one project to the next due to accessibility, terrain, difficulties affecting the penstock, etc.

However, this table provides a useful overview of where the money is spent. An axial turbine does not need a tube system like a Pelton turbine. On the other hand, the civil works are much more complex and therefore more expensive. All these factors influence the final price of the hydropower scheme, and they all depend on the natural conditions of the site. Therefore, hydropower turbines are not standardized products. The cost estimation is "flexible" to some extent.

\subsection{Legal Framework}

The legal feasibility for turbine design and construction concerns patents and protected designs. The technical principle of a hydropower machine is available in the public domain. 
Technology or ideas whose intellectual property rights have expired or been forfeited, are in the public domain. This means that the principle is available to everyone and for free. Since copyright and intellectual property laws are mostly national laws, this issue is complex, as seen in current global debates. However, physical principles like the axioms of Newton or the Bernoulli equations are available in the public domain, and the intellectual property rights have expired worldwide. Therefore, the design and publication of a hydropower impulse turbine are possible. Of course, this is true only under the precondition of not infringing on an already existing patent, which might cover a special sealing principle, for example. This is valid especially for Michell Banki turbines, which run with a very simple working principle that can hardly be patented.

\subsection{Environmental Considerations}

In addition to the decentralized, small-scale advantages of hydropower, as well as their ability to reconnect humans to nature, hydropower can be seen as an environmentally friendly renewable energy solution. Hydropower does not release $\mathrm{CO}_{2}$ during operation, nor does it emit radioactivity. Apart from the rearrangement of the resource, it is absolutely environmentally friendly. Unfortunately, the rearrangement is mostly done through dams and tubing systems. The impact on the environment here depends strongly on the site. A dam cuts of the natural flow of water and, most of the time, hinders fish migration. It is important to take these impacts into account and consider the installation of a fish pass, which enables fish to migrate in both directions.

Hydropower works with water. Water is perhaps the most important natural material and is absolutely non-toxic. A hydroelectric scheme is essentially not a consumer of water. Diverting a river into a canal or a mountain stream into a pipe may not greatly change the 
total flow, but it can have a marked effect on the environment. The rerouted water flow obviously has an impact on the environment. This is especially visible in large hydropower installations, where enormous amounts of water are rerouted. To lower the environmental impact, small-scale solutions are appropriate. Additionally, using only a part of the natural water flow for the hydro turbine lowers the environmental impact, as well.

\subsection{Energy Storage and Generators}

So far, this study has focused on renewable energy solutions that use a generator or alternator to transform the mechanical energy into electrical energy. Alternators are especially developed for battery charging and work without a regulating system (Brückmann, 2007: 27). Generators need a regulation system, which makes the whole system more complicated and more expensive.

Alternators and generators are available in diverse designs and capacities. As we have seen, it is also possible with the appropriate excitation to use a motor as a generator. A standard motor or generator is a very cost-effective solution for the electrical part of the machine. They are available worldwide and also secondhand. For example, alternators are used in cars in order to charge the battery. Thus, using an already existing technical solution might be a good start.

However, a DIY alternator or generator would complete the DIY approach. Particularly through the development of wind turbines, there are also some designs available for DIY alternators that run with permanent magnets. This could be an interesting field of research - a DIY solution for the electrical part of the machine. Unfortunately, the options for storing energy are very low. Battery banks, which have to be purchased, are used for this purpose. 
For higher capacity energy production, there are also other energy storage solutions available and in use. Heat accumulators and pump storage systems might also be interesting for smallscale decentralized energy supply solutions. These principles have to be developed in more detail and could complement the intention of using decentralized renewable energy on the consumer side. The focus of this study is on energy transformation, the mechanical side. The storage and electrical possibilities are not developed in more detail in this research project.

\subsection{Organizational Feasibility}

From an organizational perspective, designing and manufacturing a prototype should be possible with very limited organizational background, perhaps with only a few people. The second step, then, would be finding a group of people and creating a common space where the design could be tested in different environments. For this step, it is important that the design and information can be accessed and exchanged easily and worldwide. A website and collaboration tools are very important for these tasks. Regarding the workload for creating the collaboration framework, it will be helpful to find additional people with specific skills. At that time, funding may become important, as well. With regards to funding, the organizational framework should be legally defined, perhaps as a not-for-profit organization, which would help establish basic trust in the project. The organizational condition for that endeavor is based on creating a framework for collaboration and initiating the first design, which will enable people to develop and exchange experiences. 


\subsection{Scheduling}

Scheduling a turbine development is a very difficult task. If the facility and appropriate tools are available, roughly a month should be enough time to design and build a small banki turbine. Testing and optimizing will take much longer, and are not possible to define beforehand since there is no experience of efficiency and/or mechanical problems. These problems show up after the machine is built, during the testing period. These problems provoke a continual improvement process (CIP). The CIP ideally will occur in parallel with the implementation of the first machines, which provides another very important feedback loop of experience. The feedback from the actual field site where the machine is used, will be one of the most important sources for project development and optimization. Also, this feedback loop includes the most important factor - the human beings running and maintaining the energy, and last but not least, using the energy. The energy use could also lead to technical problems that may not have been seen or thought about previously in the testing environment. For that reason, it is very important to do research in an artificial laboratory, but also in the field - a "field laboratory" with several machines. Optimally, the field-testing would cover different sites over a period of at least a year.

Ideally, this will be done simultaneously among different groups around the world. If already in the prototyping phase, decentralized experience would be exchanged, which would significantly accelerate development and design optimization.

\subsection{Overall Feasibility and Conclusion}

There are many approaches to rural electrification, and the selected solution strongly depends on the natural characteristics of the site. This is quite obvious in hydropower, where the 
selection of the turbine depends on the local characteristics of the water flow. Most of the technologies seem feasible for a DIY approach, and some of them are already available, albeit in a limited way.

Analyzed and investigated thoroughly for the advantages and disadvantages of different renewable energy technologies, the decision was in favor of hydropower for rural electrification. withdrawing from all of the positive and negative aspects related to the different turbine types, the result of this research study demonstrated that the Michell - Banki turbine is a feasible turbine type for a DIY open source hardware design. The next chapter, Practical Application: Pico Cross Flow describes the technical and social processes of an Open Source Turbine for rural electrification. This includes the legal and social structures of finding and founding an NGO as a legal entity with the aim to develop and share an open design. In addition, the technical process, from design and calculation to prototyping and testing, will also be covered. 


\section{Practical Application: Pico Cross Flow}

The following pages will describe the process of the technical design of the Pico Cross Flow as well as the open source documentation and the launch of an open source community.

\subsection{How to Start such an Endeavor?}

As we have seen in the previous sections, there is a huge demand for rural electrification solutions, and as we have seen in the evaluation analysis matrix and the SWOT analysis, there is great potential for small-scale hydropower machines. An action plan and a roadmap are necessary. The arising question is now, where and how can such a project get started? Partly, this question was answered already with the SWOT analysis strategy. This section will focus on the more specific actions and a roadmap. Drawing on the SWOT analysis, the actions are divided into the following four categories:

\section{A) Becoming visible campaign (website):}

The first step is to publish the idea and make it visible to the world. By doing so, people might join and support the idea.

The concrete action is creating a website, promoting it and the concept. The first step was to find a name for the endeavor. Several ideas were evaluated and the decision was made to name the project: Pico Cross Flow. This name includes the turbine type and in the name of 'Pico' the definition of the size of the turbine. Additionally the domain name picocrossflow.com was available. 
After having a name it was possible to design a logo for the project. Hector Flores, a designer and artist from Managua, Nicaragua elaborated the final logo and design. Finding a name, registering the website and having a logo was followed by a description of the project and the publication of the website, which is online at www.picocrossflow.com and was implemented with the open source framework "Drupal 7". The technical process of how I designed, programmed and published the website is not part of this dissertation and will not be described here. Some parts of the website are visible in the Annex of this dissertation. The complete outcome is available online at www.picocrossflow.com.

\section{B) Forming a legal entity:}

At the same time it was important to form a legal entity to receive funding and having a clear legal status for further actions. For that reason, putting the idea into the legal framework of an NGO is a precondition and one of the main priorities at this point.

The concrete action was getting information about the legal requirements of founding an NGO. After some research I decided to create the legal entity in form of a "not-for-profit association" according to German law registered in Germany ${ }^{51}$. This was done through an official foundation assembly and where invited people agreed on an association statute. This assembly was taking place on February 12, 2012 in Titisee-Neustadt. The legal entity then was officially formed and registered on April 20, 2012 at the district court in TitiseeNeustadt.

\section{C) Funding (crowd funding campaign):}

A very important part is to get initial funding and a testing facility. For an initial start of such a project, it is important to have the necessary initial financial liberty to invest appropriate

\footnotetext{
${ }^{51}$ The legal entity is called Global Anchor e.V., registered in Titisee-Neustadt (Vereinsregister Titisee-Neustadt, Registriernummer 467)
} 
time. This would support a fast development process, which would also make the overall project more attractive. For the funding process, a legal framework is necessary and was done in the form of Global Anchor e.V..

Unfortunately the funding of the project was not taken very serious. More than 90 percent of the overall project expenses were funded by the author.

\section{D) Design, prototyping and creating a collaboration platform:}

The first design is a "virtual" design. There is no end-user identified, and the first design aims to be installed in a testing facility, not in the field. Therefore, the machine inputs (head and flow) have to be defined. With this definition, the technical solution has to be designed and prototyped. All of this has to be published and shared from the beginning on. If there are enough people involved and the information is not only exchanged one-way, a participation platform has to be created. This strongly depends on the number and activity of people involved.

Looking back, this has been a difficult task and was not accomplished as expected. It was very difficult to inspire people with the project. From my experience, many people were interested from the beginning on, but coming to real work and tasks the output was very limited. This seems to change since the finished prototype was tested at the technical University of Vienna. By having a "finished" product more and more people became interested and are planning to manufacture the turbine in several countries now. The knowledge platform regarding exchange of pico hydro power in general is still pending, since the focus was clearly on the design on the Pico Cross Flow.

These four measures had to be taken simultaneously. It was important to become visible, have a legal framework and a technical solution at the same time. The derived first actions 
were creating a website, founding an NGO and defining the design parameters for the prototype. The timetable, charts and milestones are not included in this dissertation, since it is focusing on the technical outcome, which is visible in the following pages and the Annex.

\subsection{The Pico Cross Flow Turbine, an Open Source Hardware}

\section{Design}

The initial design parameters were defined out of my experience with natural conditions at pico hydro power installations. I defined the natural preconditions for the turbine as follows:

\begin{tabular}{|l|l|l|l|}
\cline { 2 - 4 } \multicolumn{1}{c|}{} & ranging from & design & ranging to \\
\hline \hline Head [m] & 8 & 20 & 30 \\
\hline Flow [1/s] & 20 & 20 & 40 \\
\hline Power [kW] & 0.5 & 2 & 3 \\
\hline
\end{tabular}

This seemed to be a reasonable condition range, which can be found in nature and literature and at the same time provides with a decent amount of energy. For a specific design the head of $20[\mathrm{~m}]$ and a flow of $20[1 / \mathrm{s}]$ the hydraulic calculation was made as described in the following chapter. For easier understanding, the described design is simplified and the whole calculation with results can be found in the Annex of this dissertation. 


\subsubsection{Hydraulic Calculation and Design}

For the hydraulic design a research on the layout principles was made and several works on Cross Flow turbine designs were compared. The underlying design principles are based on the theory of Donat Banki, who patented this novel concept around 1920 (Arter \& Meier, 1990: 6). His publication was reproduced in a free translation, published in 1949 by Mockmore \& Merryfield at to Oregon State College in the USA (Mockmore \& Merryfield, 1949). This publication contains the full theory of Donat Banki and is the basis for the runner design of the Pico Cross Flow. In addition to that, further theoretical and design aspects (blade geometry, inlet curve) according to Arter \& Meier (1990) are used. The comprehensive calculation, including the results for the Pico Cross Flow design, is available in the annex of the dissertation. The following pages depict the basic cross flow runner and inlet theory used for the design layout.

As already mentioned in previous chapters, the potential energy in water depends on the main two factors head and flow. The available head is defined as the altitude difference between the top and the bottom of the penstock, as visible in figure 7.2. The speed of water depends on the available head. "The higher the head, the higher the speed of water" (Gnecco, 2010: 3). With the speed and flow the estimated power output can be defined. Power is defined as the product of water flow and head (height difference between the intake and the turbine):

$$
\begin{array}{lll} 
& \text { where: } & \\
& \mathrm{P}=\text { Power } & {[\mathrm{W}]} \\
& \mathrm{Q}=\text { Flow of Water } & {\left[\mathrm{m}^{3} / \mathrm{s}\right]} \\
& \mathrm{H}=\text { Head } & {[\mathrm{m}]} \\
& \mathrm{g}=\text { Gravity }(9.81) & {\left[\mathrm{m} / \mathrm{s}^{2}\right]} \\
& \eta=\text { Efficiency } & {[\%]} \\
& \mathrm{r}=\text { Density of Water }(1000) & {\left[\mathrm{kg} / \mathrm{m}^{3}\right]}
\end{array}
$$


As visible in equation 7.1, both, head and flow have the same influence on the potential, which offers the selected site. The other influencing factors are constants with fixed values. For very small schemes and because of simplification it is possible to use the following equation as a first approach (taking an efficiency of about $35-55 \%$ into consideration):

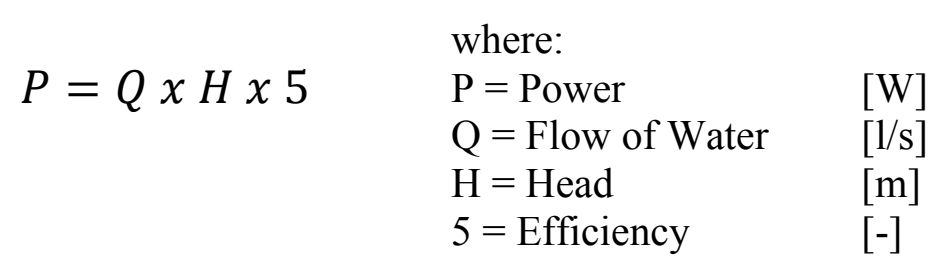

With equation 7.2 and the given design values (head $=20 \mathrm{~m}$ and flow $=20 \mathrm{l} / \mathrm{s}$ ) the expected turbine output will be at 2 kilowatts.

The flow is defined as the amount of water, which passes through the penstock in a specific time and therefore is defined as the product of speed of water and area of the cross section of the penstock, as visible in equation 7.3.

$$
Q=A x c\left[\mathrm{~m}^{3} / \mathrm{s}\right]
$$

To reduce friction losses, the flow in the penstock has to be as slow as possible. For the design of the penstock the practical application would be finding an adequate ration between big diameter (high costs) and efficiency loss (small diameter) of the penstock. With the speed and the flow the diameter of the penstock can be defined, where:

$$
A=\frac{d^{2} x \pi}{4}\left[\mathrm{~m}^{2}\right] \text { furthermore } d=\sqrt{\frac{4 x Q}{c x \pi}}[\mathrm{m}]
$$

The selection of the turbine diameter is a trade-off between costs and friction losses. With the focus on the design of the turbine, the dimensioning of the diameter of the penstock is not 
part of this dissertation. Furthermore the friction losses and costs of the penstock highly also depend on its length. So it is more reasonable to do the penstock design and calculation at the specific site.

To define the absolute speed of water the following equation can be used:

$$
\begin{array}{lll}
c=k \sqrt{2 x g x H} & \text { where: } & \\
& \mathrm{c}=\text { Speed of water jet } & {[\mathrm{m} / \mathrm{s}]} \\
& \mathrm{k}=\text { Nozzle coefficient } & {[-]} \\
& \mathrm{g}=\text { gravitational acceleration }(9.81) & {\left[\mathrm{m} / \mathrm{s}^{2}\right]} \\
& \mathrm{H}=\text { Head } & {[\mathrm{m}]}
\end{array}
$$

In literature a specific coefficient named $\mathrm{k}$ was defined. Its value depends on the geometrical characteristics of the nozzle. According to Mockmore \& Merryfield (1949: 10) the value of k equals to 0.967 . Sketch 7.2 depicts the gross head of a hydroelectric scheme. With these

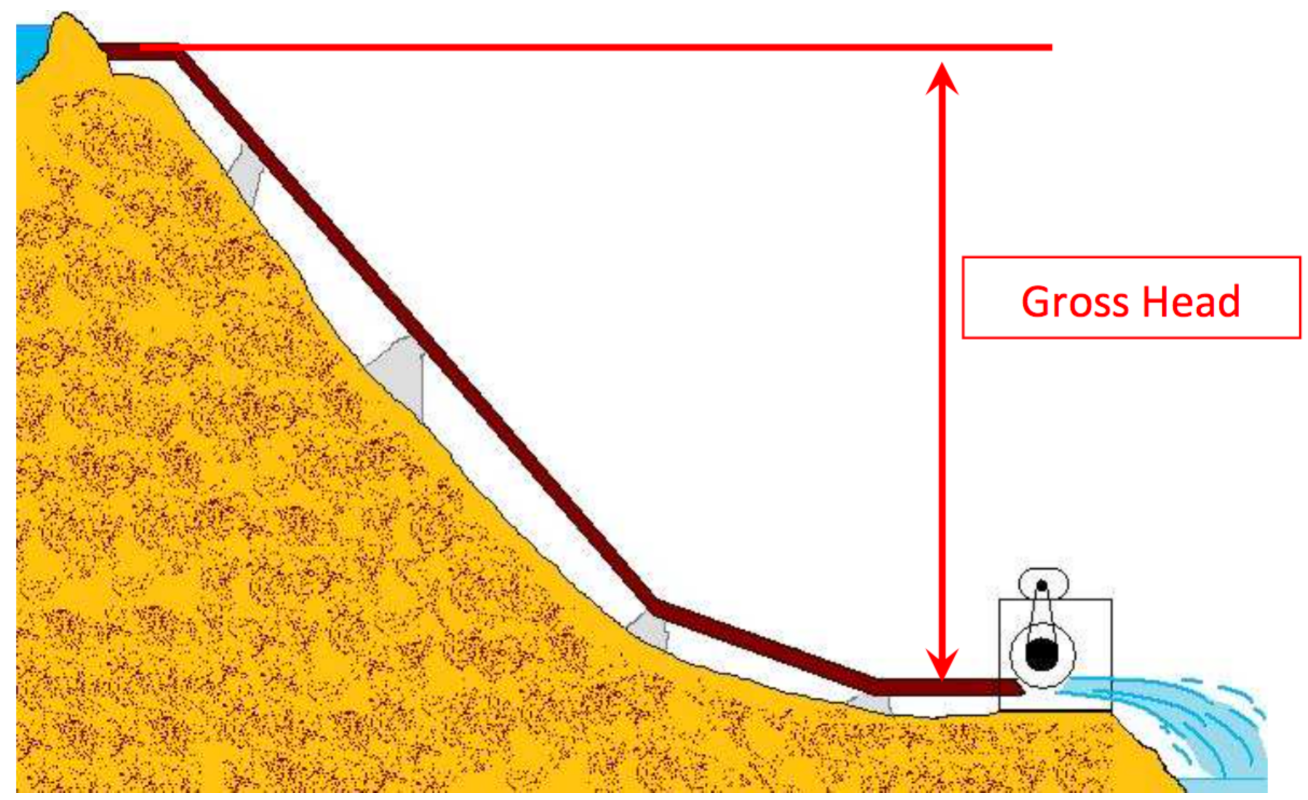

[7.2] Gross Head of Hydro Power Scheme (Gnecco, 2010: 3)

values it is possible to define the turbine optimal speed and dimensions. According to literature and velocity diagram the rotational speed of the turbine can be calculated according to the following equation (Mockmore \& Merryfield, 1949: 10): 


$$
\begin{array}{lll}
u=\frac{1}{2} x \operatorname{cx} \cos \alpha & \begin{array}{l}
\text { where: } \\
\mathrm{u}=\text { Turbine rotational speed }
\end{array} & {[\mathrm{m} / \mathrm{s}]} \\
& \mathrm{c}=\text { Absolute velocity of water } & {[\mathrm{m} / \mathrm{s}]} \\
& \alpha=\text { Absolute velocity angle } & \left.{ }^{\circ}\right]
\end{array}
$$

The turbine rotational speed and diameter define the revolutions of the turbine, as we can see in the following equations:

$$
\begin{aligned}
& w=2 \times \pi \times n \quad \text { where:- } \quad \text { w }=\text { Angular speed } \quad[1 / \mathrm{s}] \\
& u=r x w \quad \text { where: } \\
& \mathrm{r}=\text { Radius of turbine runner } \quad[\mathrm{m}]
\end{aligned}
$$

Combining these two equations, eliminating the angular speed and solve the equation according to the revolution we get the direct relation of the turbines diameter with the turbines revolution:

$$
n=\frac{60 \times u}{\pi \times D} \quad \begin{array}{ll}
\text { where: } \\
\mathrm{D}=\text { Diameter of Turbine }
\end{array}
$$

Depending on the electrical frequency and the amount of poles in the generator a specific speed of the generator is required. This necessary revolutionary speed of the generator can be adjusted (in a limited range) accordingly to the diameters of the belt transmission. For simplifications reasons it is assumed, that the turbine speed should be at least 1000 rounds per minute. With this revolution it is ensured, that a generator with a low number of poles would work satisfying.

By having defined the turbines diameter in relation to the available water head the next step is to define the turbines width, in relation to the amount of water flow. To do so a modification 


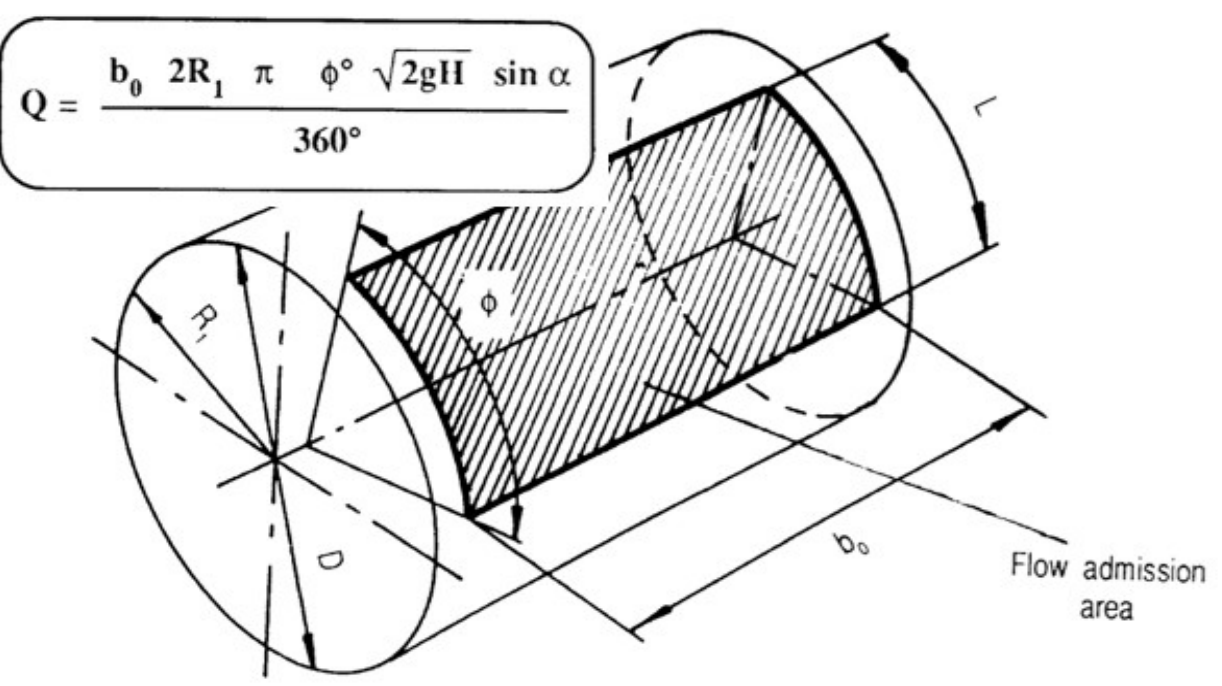

[7.3] Flow Admission Area of a Cross Flow Turbine (Arter \& Meier, 1990: 28)

of equation 7.3 is necessary to adjust the flow admission area. The corresponding flow admission area of water is depicted in the previous sketch 7.3.

According to equation 7.3, with the adaption of the area and solved according to the runner width, we get the following equation:

$$
b=\frac{Q \times 360^{\circ}}{D x \pi \times \phi^{\circ} \times \sin \alpha \times \sqrt{2 \times g H}} \quad \begin{array}{ll}
\text { where: } \\
\text { b= Runner width } & {[\mathrm{m}]} \\
\Phi=\text { Admission arc } & {\left[{ }^{\circ}\right]}
\end{array}
$$

With the defined water head and water flow we were able to calculate the turbines revolutions and the runner dimensions (diameter and width).

The next step is to define the geometry of blades and the inlet curve of the water flow. The blade geometry can be defined according to Arter \& Meier (1990: 23) with the following equations, depicted in figure 7.4 and 7.5. The goal is to define the pitch circle radius. The curvature radius of the blades is positioned on the pitch circle radius. The application of the 
necessary equations for the pitch circle diameter calculation, depicted in 7.4 , can be found in the Annex of this dissertation.

$$
\begin{aligned}
& \mathbf{c}=\sqrt{\mathbf{R}_{1}{ }^{2}+\mathbf{R}_{2}{ }^{2}-2 \mathbf{R}_{1} \mathbf{R}_{2} \cos \left(\beta_{1}+\beta_{2}\right)} \\
& \varepsilon=\arcsin \left[\frac{\mathbf{R}_{2} \sin \left(\beta_{1}+\beta_{2}\right)}{\mathbf{c}}\right] \\
& \xi=180^{\circ}-\left(\beta_{1}+\beta_{2}+\varepsilon\right) \\
& \phi=\beta_{1}+\beta_{2}-\left(180^{\circ}-2 \xi\right) \\
& \mathbf{d}=\frac{\mathbf{R}_{1} \sin \phi}{2 \sin \left(180^{\circ}-\xi\right)} \\
& \delta=180^{\circ}-2\left(\beta_{1}+\varepsilon\right) \\
& \mathbf{r}_{b}=\frac{d}{\cos \left(\beta_{1}+\varepsilon\right)} \\
& r_{p}=\sqrt{r_{b}{ }^{2}+R_{1}{ }^{2}-2 r_{b} R_{1} \cos \beta_{1}}
\end{aligned}
$$

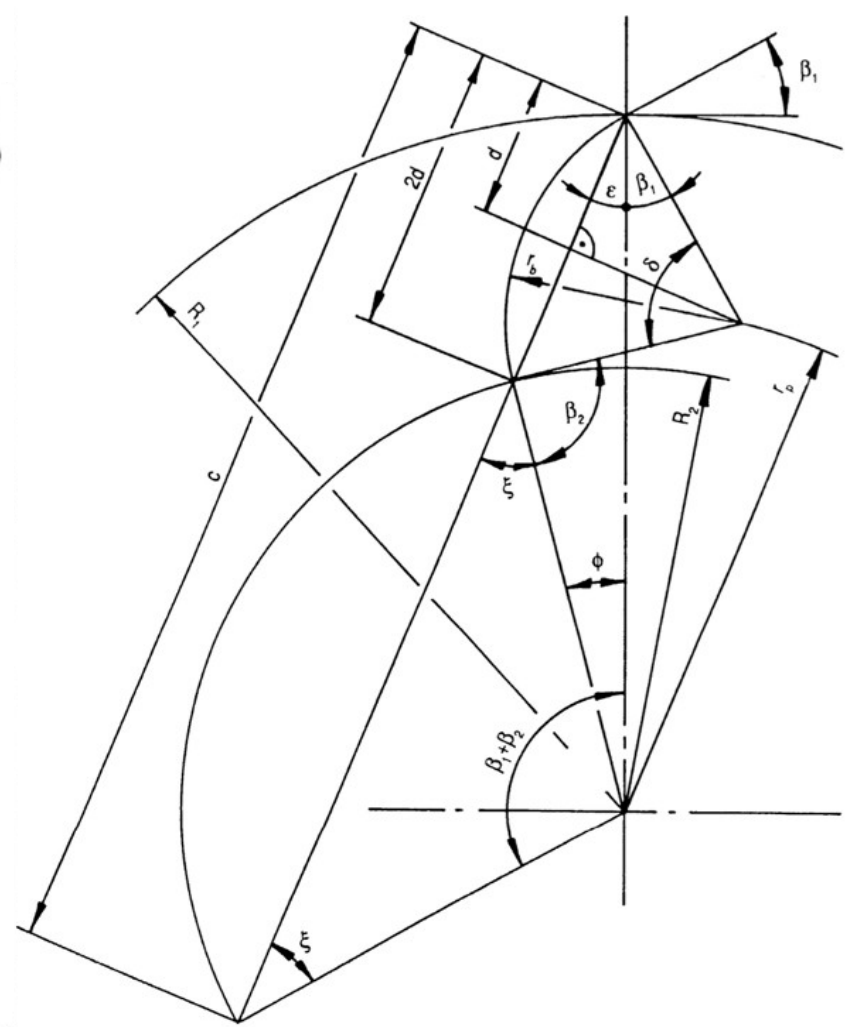

[7.4] Equations of Geometrical Relationship between Parameters and [7.5] and Construction of Blade Geometry (Arter \& Meier, 1990: 24 and 23)

The last missing value is the inner diameter of the runner, which can be defined by the relationship between the outer and inner diameter of the runner, which is assumed to be around 0.66 depending on the source.

With the calculation of the pitch circle radius and the application of the diameter relationship, all blade geometries are defined. At this point it is important to check the calculated values according to plausibility with the help of velocity triangles. 
The velocity diagram, or velocity triangle is a vector illustration of the various velocity components of the working fluid in a turbine. In this triangle the relationship between the relative, peripheral and absolute velocity is defined as:

$$
\begin{array}{lll}
c=u+w[m / s] & \text { where: } & \\
& \mathrm{c}=\text { Absolute velocity } & {[\mathrm{m} / \mathrm{s}]} \\
& \mathrm{u}=\text { Peripheral velocity } & {[\mathrm{m} / \mathrm{s}]} \\
& \mathrm{w}=\text { Relative velocity } & {[\mathrm{m} / \mathrm{s}]}
\end{array}
$$

With the help of the velocity triangle it is possible to compare the working fluid velocities at the entrance of the turbine with the velocities at the exit of the turbine. Energy exchange from the moving fluid to the turbine takes place if the "entrance velocity triangle is different from the exit velocity triangle" (Arter \& Meier, 1990: 4). According to Euler:

The precondition for an energy exchange between a moving fluid and a moving runner blade of a hydraulic machine is that the runner blade causes the fluid to change its velocity. In the case where the fluid is accelerated by the runner blade energy is imparted by the runner blades to the fluid, as happens in pumps. In the opposite case, where the fluid is retarded by the runner blade, energy is imparted to the runner of the machine by the moving fluid, which is the operating principles of all water turbines (Arter \& Meier, 1990: 4).

A typical velocity triangle of a Cross Flow turbine runner is shown in figure 7.6. The commonly accepted conventions are visible in this sketch, where index 1 indicates the channel inlet and index 2 indicates the channel outlet. In this sketch the angles between the velocity arrows are also visible. These angles are important, because they define the size of the velocities. Several attempts have been made in history to optimize these angles. Significant assumptions can be made and used for the Pico Cross Flow design as well, since they have been investigated sufficient. These angular assumptions are depicted in the calculation results as well in the attached calculation of the Pico Cross Flow turbine. 


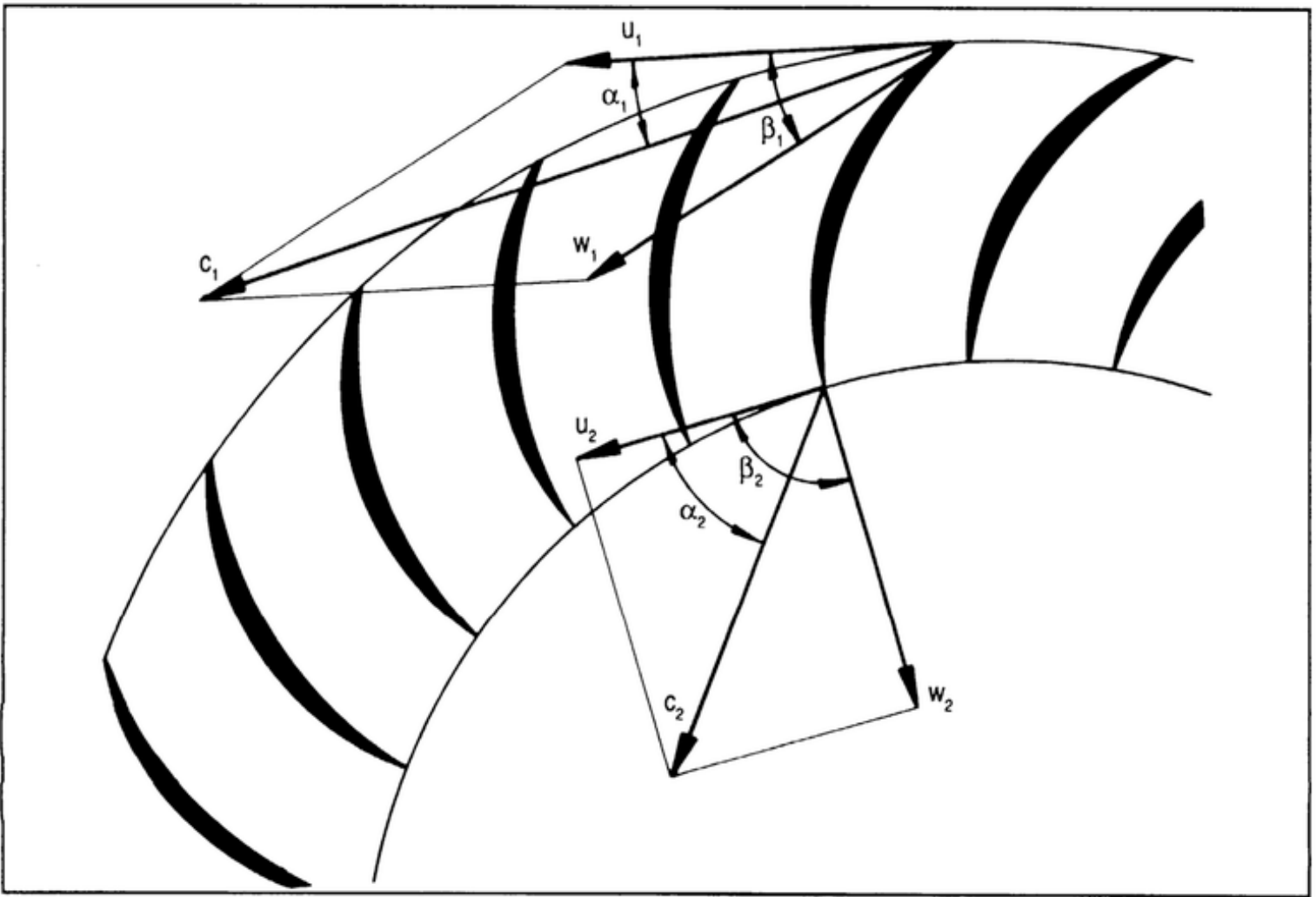

[7.6] Velocity Triangles in a Runner with peripheral Blades (Arter \& Meier, 1990: 3)
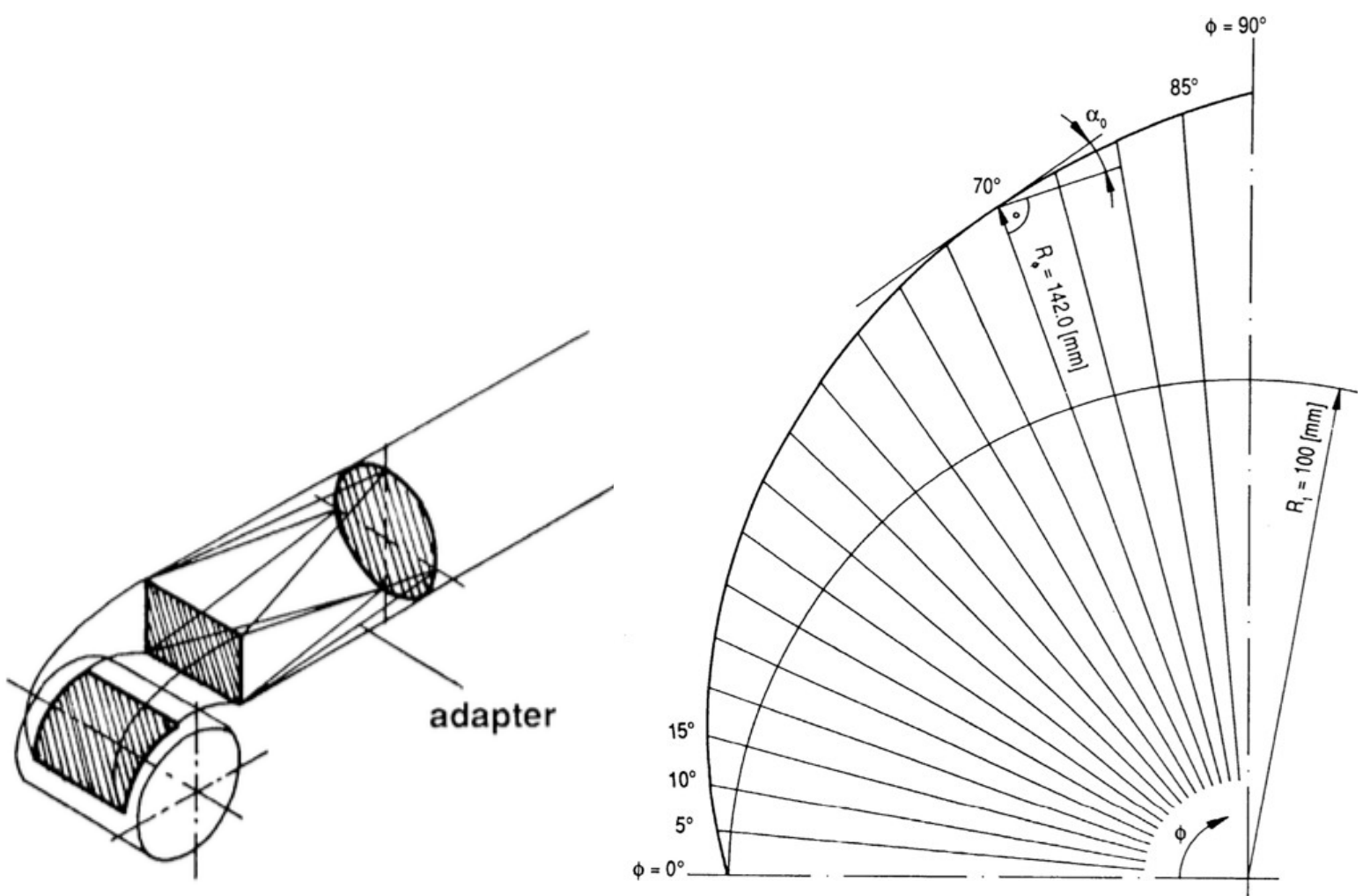

[7.7] Cross Sections of Flow at the Inlet and [7.8] Design of the logarithmical Inlet Spiral (Arter \& Meier, 1990: 25 and 26) 
The next step is the definition of the injector dimensions. In sketch 7.7 it is very visible, that the water flow at the inlet of a Cross Flow turbine transforms from a circle to a rectangle shape. To reach a high efficiency level it is desired that all stream-lines have the correct velocity and angle of admission to the runner. According to Arter \& Meier, the ideal flow conditions result in a logarithmical inlet spiral, which is exemplary depicted in figure 7.8. For the Pico Cross Flow hydraulic design this approach was used and the calculation of the logarithmical spiral is attached in the Annex of this dissertation.

In the design and the calculation process of the Pico Cross Flow turbine the assumptions depicted in table 7.9 have been made.

\begin{tabular}{|l|l|}
\hline \multicolumn{2}{|l|}{ Assumptions } \\
\hline Absolute velocity angle $\alpha$ & $16\left[^{\circ}\right]$ \\
\hline Relative velocity angle (entrance) $\beta_{1}$ & $30\left[^{\circ}\right]$ \\
\hline Relative velocity angle (exit) $\beta_{2}$ & $90\left[^{\circ}\right]$ \\
\hline Diameter ratio & $0.66[-]$ \\
\hline Number of blades & $26[-]$ \\
\hline Runner admission angle & $90\left[^{\circ}\right]$ \\
\hline Runner outer diameter & $120[\mathrm{~mm}]$ \\
\hline Turbine speed more than & $1000[\mathrm{rpm}]$ \\
\hline
\end{tabular}

[7.9] Calculation Assumptions and geometrical Dimensions (author's illustration)

With these assumptions the geometrical calculation was performed. The geometrical results of the calculation are visible in table 7.10 .

\begin{tabular}{|c|c|}
\hline Results & \\
\hline Turbine speed at design head of $20[\mathrm{~m}]$ & $1477[\mathrm{rpm}]$ \\
\hline Geometrical Dimensions & \\
\hline Runner outer diameter & $120[\mathrm{~mm}]$ \\
\hline Runner inner diameter & $80[\mathrm{~mm}]$ \\
\hline Runner width & $60[\mathrm{~mm}]$ \\
\hline Pitch circle radius & $44[\mathrm{~mm}]$ \\
\hline Blade curvature radius & $19[\mathrm{~mm}]$ \\
\hline Segment angle on blade $\delta$ & $73\left[^{\circ}\right]$ \\
\hline
\end{tabular}


With these assumptions based results the engineering and design of the physical turbine matching the hydraulic conditions was possible. This manufacturing process is described in the following chapter.

\subsubsection{Manufacturing a Prototyp}

With the results of the hydraulic design the main dimensions of the turbine and the inlet were defined. By comparing different Cross Flow turbine designs the decision was made to use the design of the Tp100 from ENTEC Indonesia as a basis for the mechanical layout (ENTEC, 2009). Conversations with the former CEO of ENTEC Indonesia Gerhard Fischer encouraged me with this decision. Gerhard Fischer's expert knowledge is due to the fact that he had worked with Cross Flow turbines the last 30 years and gained also profound experience in rural electrification.

With this input I was searching for an adequate workshop in Managua, Nicaragua to manufacture a prototype. After visiting several workshops I was glad to find Bernhard Reichel, who runs a small engineering business and is a brilliant engineer. With the input of Gerhard Fischer and the mechanical skills of Bernhard Reichel I was able to manufacture the prototype of the Pico Cross Flow turbine in December 2014. The following pictures show some of the manufacturing process. Further images are available in the Annex of this dissertation and on the website www.picocorssflow.com.

The difficulties and challenges of the manufacturing process are not described in the dissertation. However these experiences are incorporated in the design of the subsequent prototype. 

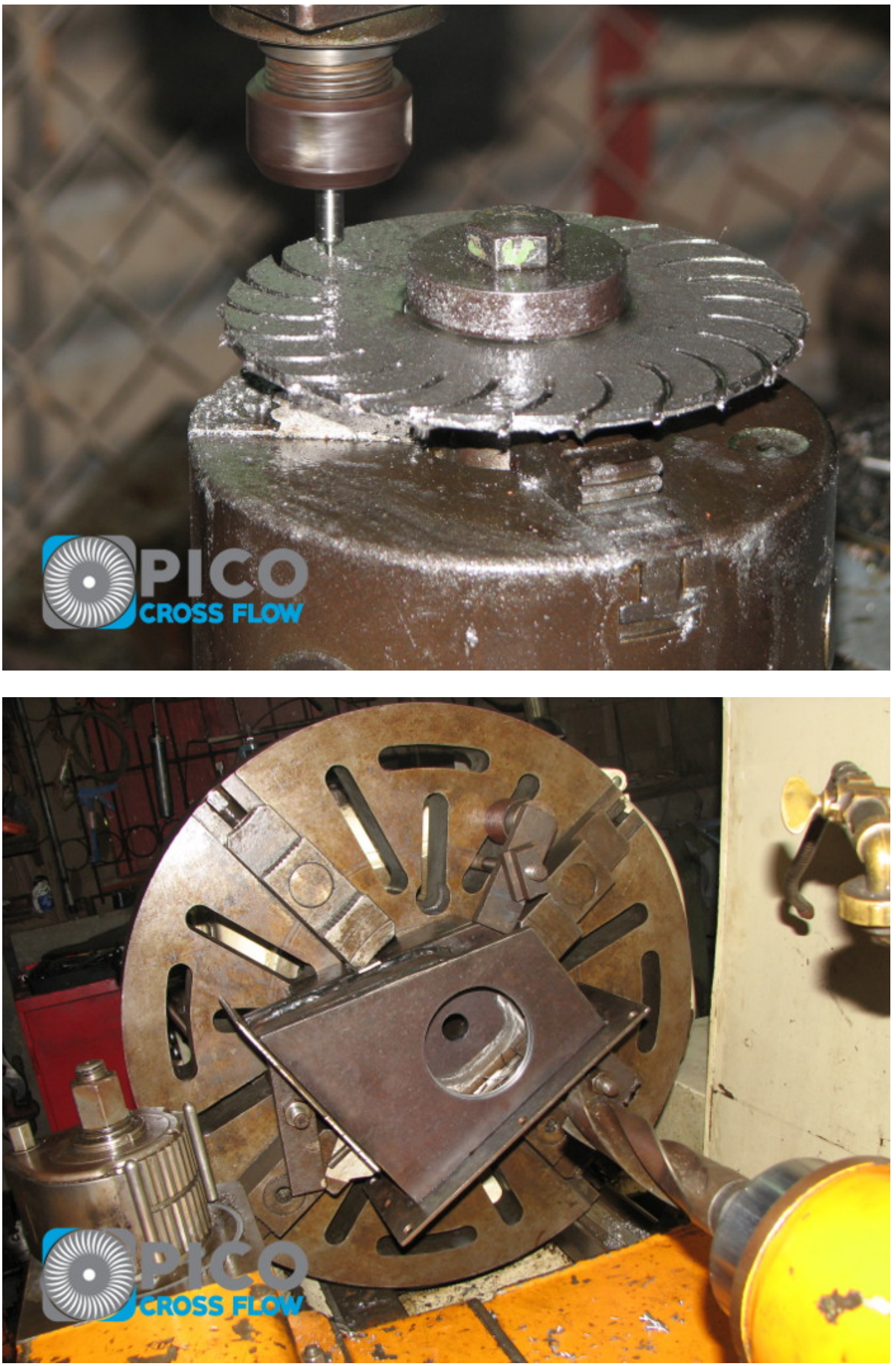

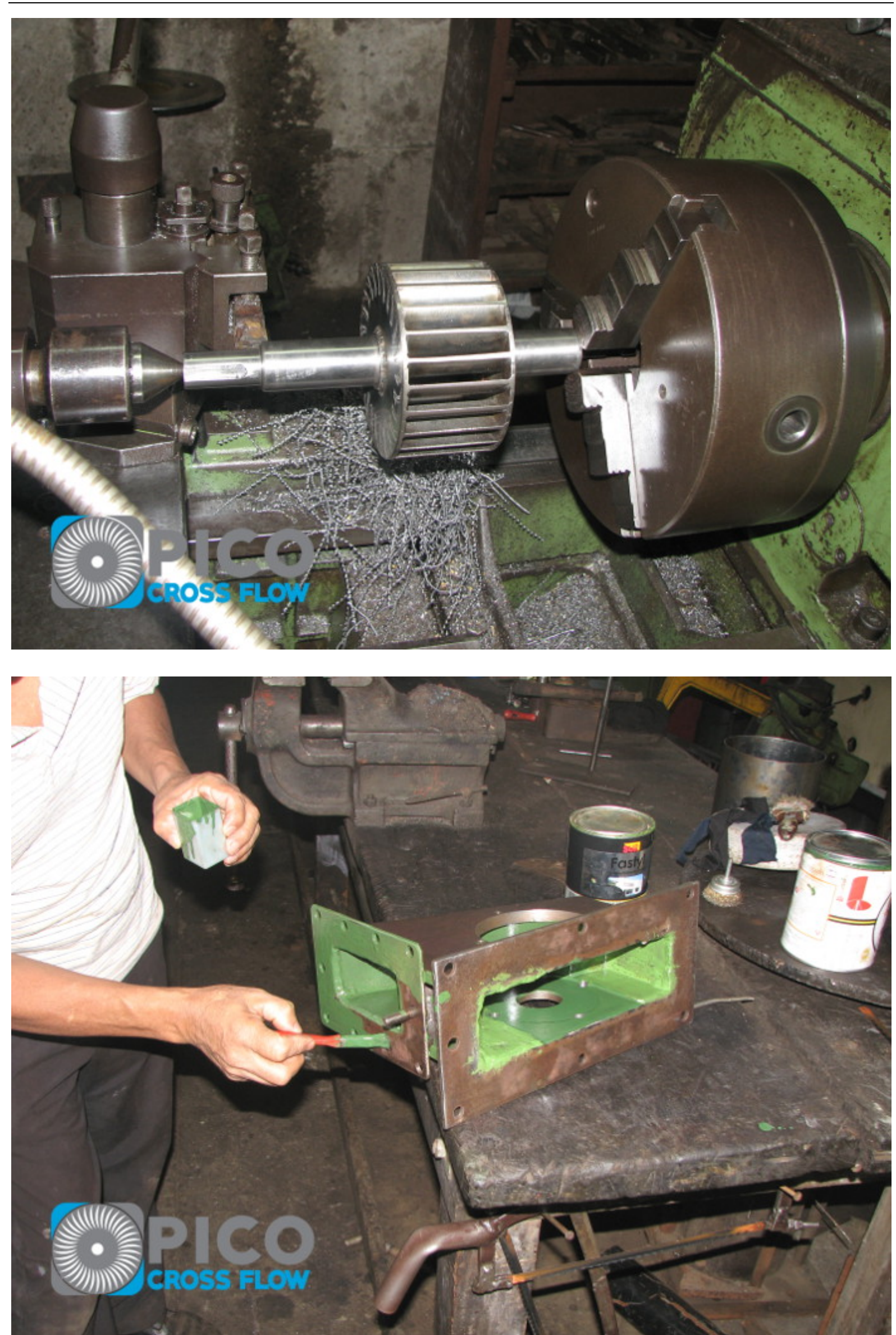

[7.11] Manufacturing of the Prototype in Managua (author's pictures) 


\subsubsection{Hydraulic Testing of the Prototype}

With the finished prototype the search for an adequate site to do hydraulic testing began. I was hesitant to publish the design on the Internet without knowing the performance of the turbine. Unfortunately I was not able to find a site, which was accessible and at the same time in a physical condition to set up a test rig without the investment of a huge amount of money.

After some time the search was directed towards Universities, which are equipped with the necessary hydraulic laboratories to perform hydraulic turbine testing. In the frame of this research I came in contact with Professor Christian Bauer from the technical University of Vienna. In a meeting in Vienna I was offered to do the testing of the Pico Cross Flow prototype in the frame of a Bachelor thesis at the hydraulic laboratory at the University of Vienna. In late 2015 I was writing a tender, describing the task and the desired outcome to find a student, who was willingly to design a test rig for the turbine. In spring 2016 Adam Malik decided to finish his Bachelor studies with this task. Sketch 7.12 on the following page depicts the setup of the test rig, where the testing was undertaken.

In July 2016 I was able to participate in the testing in Vienna. Several heads were tested and the flow rates and efficiencies were defines as well as the runaway speed. With the usual difficulties, which arise in such a testing period I gladly received the test results in October 2016.

Unfortunately the test results were not as good as the expected outcomes. The turbines behavior at a broad head and flow range is almost stable, which is a very positive result. But the overall efficiency hardly reaches more than 50 percent. Table 7.12 shows turbine speeds and power outputs for different heads. The complete test results, including recommendations 
for the mechanical design, can be found in the Bachelor thesis of Adam Malik (Malik, 2016).

The main test results and core findings are depicted in the annex of this dissertation.

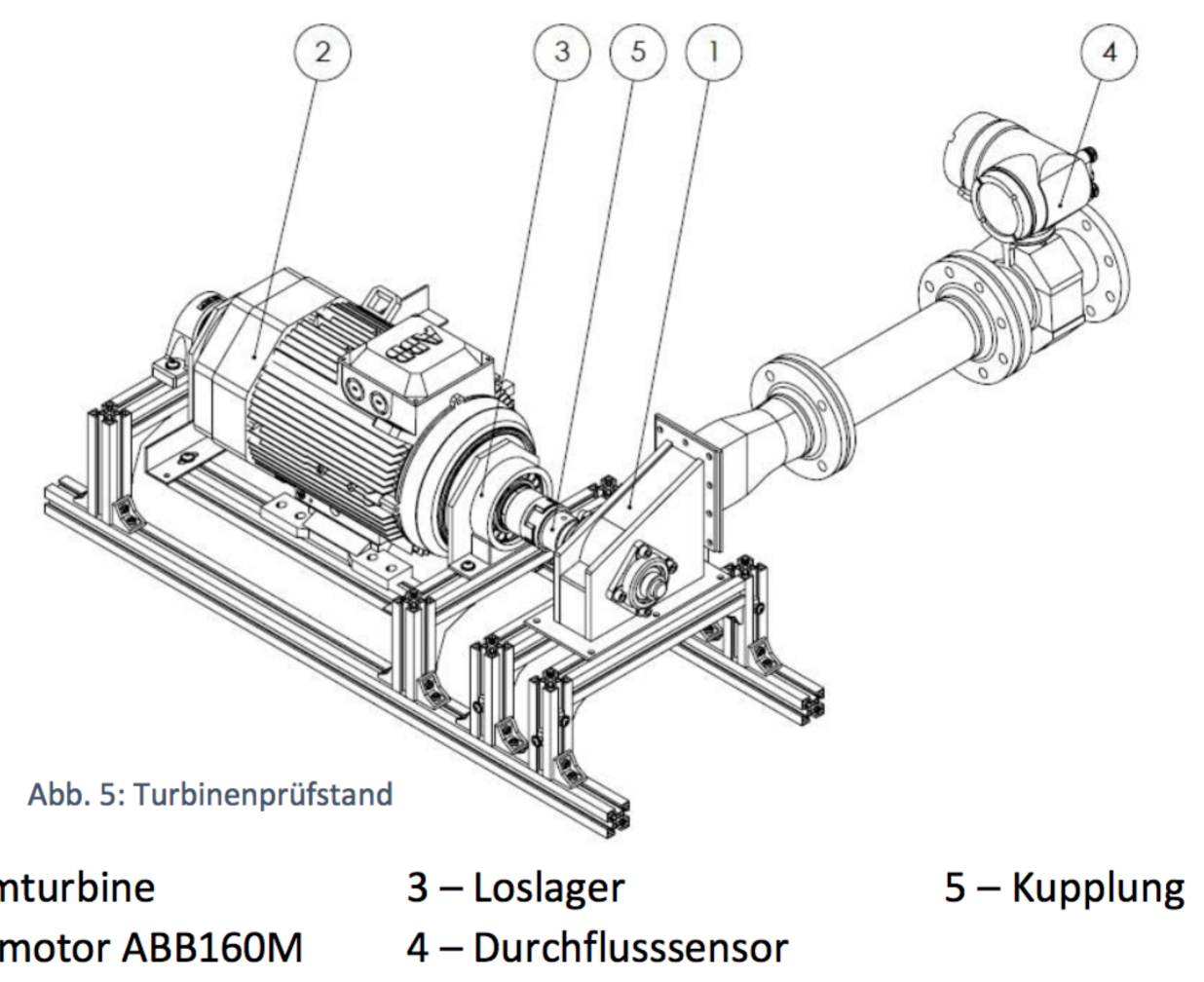

1 - Durchströmturbine

2-Asynchronmotor ABB160M

4 - Durchflusssensor

[7.12] Turbine test rig at the University of Vienna (Malik, 2016: 10)

\begin{tabular}{|c|c|c|c|c|}
\hline \multicolumn{5}{|c|}{ Optimale Drehzahlen für verschiedene Fallhöhen } \\
\hline $\mathbf{H}(\mathbf{m})$ & $\mathbf{n}_{\text {opt }}$ (U/min) & $\mathbf{P}(\mathbf{k W})$ & $\mathbf{Q}(\mathbf{l} / \mathbf{s})$ & $\mathbf{n}(\%)$ \\
\hline $\mathbf{5}$ & 755 & 0,429 & 17,75 & 49,28 \\
\hline $\mathbf{8}$ & 958 & 0,889 & 22,46 & 50,48 \\
\hline $\mathbf{1 0}$ & 1058 & 1,159 & 24,61 & 48,06 \\
\hline $\mathbf{1 2}$ & 1211 & 1,570 & 27,18 & 49,64 \\
\hline $\mathbf{1 5}$ & 1364 & 2,196 & 30,48 & 48,95 \\
\hline $\mathbf{2 0}$ & 1618 & 3,492 & 34,94 & 50,91 \\
\hline $\mathbf{2 5}$ & 1824 & 4,951 & 38,95 & 51,95 \\
\hline $\mathbf{3 0}$ & 1981 & 6,490 & 42,89 & 51,43 \\
\hline
\end{tabular}

[7.13] Different turbine speeds related to water head (Malik, 2016: 39) 
With these results the question arose: What is the next step? On one hand it would have been great to have a significant increase of the efficiency, up to 70 percent. I discussed it with Prof. Bauer and was offered that in an additional Bachelor thesis the task of optimizing the efficiency could be worked on. On the other hand it would have been nice to have some more information on the website to empower people to finally manufacture copies of the Pico Cross Flow. Doing the optimization would at least take another 6-8 months. Unfortunately the hydraulic laboratory was about to move to another building, which meant that there would be another delay of about 6 months.

My decision then was to move on both sides. On one hand prepare a tender and define a specific task for the optimization of the hydraulics and mechanical design. And on the other hand move on with the project documentation and publish all available information. This includes manufacturing drawings of the turbine, documentation, test results, photos and movies.

The next chapter describes the structure and the process of the documentation of the Pico Cross Flow turbine.

\subsubsection{The Pico Cross Flow Files - A Comprehensive Documentation}

The documentation is a core aspect of the open source idea. The documentation of the Pico Cross Flow is available at picocrossflow.com, the project website. With the development of the website the decision was made to host the information on the website itself and not use a "software as a service provider". This has been done, with the exception of the pictures and 
movie files. Pictures are published by the YAHOO service Flickr. Movie files are published with the help of the video hosting platform vimeo.

The structure of the information available was elaborated according with the Best Practices for Open Source Hardware 1.0 published by the Open Source Hardware Association (OSHWA, 2013). This document can be seen as a guideline for the publication of open source hardware documentation. The following table of content of the Pico Cross Flow Files, is the result of applying the best practices of the Open Source Hardware Association.

The documentation consists of general information about the hydro power schemes and guideline for its evaluation. The second part describes the necessary technical components of a hydro power scheme. The third part touches on the Pico Cross Flow turbine and offers several documents to support the manufacturing process. These information are followed by a installation and operation manual. The mentioned documentation is not limited to the turbine itself, but covers a broader information range attached to hydro power. Additionally there is a part where a community is invited to share their experience with reproducing the Pico Cross Flow. The idea is that this can be done with photo and video documentation in some sort of a project showroom.

The technical content as well as the community content of the preliminary structure of the Pico Cross Flow Files is not completed. In many cases the necessary information is not available yet. Nevertheless, this structure is already a guideline and direction for further publication of documents. 
Preliminary structure of the Pico Cross Flow Files:

- Site Evaluation and System Design

○ Energy Demand Evaluation

- Site Evaluation

- Technical and Economic Feasibility

- Component Selection and Design

○ Civil Works

○ Penstock

- Powerhouse

- Turbine

○ Generator

- Governing System

- Electrical Distribution

- Turbine Specification (Preliminary Prototype Specification available)

- System Overview

- Application Chart

- Bill of Materials

- CAD Model Files

- Manufacturing Drawings

- Building Process

- Licensing Information

- Getting Started

- Installation Process

- Operation

○ System Operation Manual

- Maintenance Guide

- Community

- Mailing List

- Map of Builders and Project Showroom

- Project Images

- Project Videos

- $\quad$ Other Files

- Fabrication Video

- System in operation Video

The documentation is an ongoing process and hopefully more interested people will contribute with higher motivation to Pico Cross Flow Files in the future. 


\subsection{Challenges, Next Steps and Outlook}

With the practical application of this research many things have been archived by December 2016. At the same time many more things could be archived in the future. The next steps of the project can be divided in four main branches of actions:

\section{A) Publication of the actual Project Status}

With the testing results and a clear technical solution it is important to empower people to rebuild the turbine. A necessary precondition for that is the publication of the files. Therefore a concrete action will be to create a 3D model, manufacturing drawings and a manufacturing documentation. With these files published and available online, people are able to manufacture a copy of the turbine and eventually contribute with their experience to the project.

\section{B) Design Upgrades}

Another branch of concrete action is the optimization of hydraulic and mechanical design. In the frame of another Bachelor thesis at the University of Vienna the hydraulic optimization of the turbine could be addressed. The upgrade on the hydraulic design and an increased efficiency rate will follow an adaption of the mechanical design. This process depends on the availability of the hydraulic laboratory at the University of Vienna, which is about to move into a new building. Time-wise, this might collide with action D), implementing the turbine at a pilot site.

Another option arose through a contact to the University of Stuttgart, where it seems possible to have students do hydraulic optimization through simulation. These two options have to be evaluated. 


\section{C) Funding and Finance}

Funding is important and necessary to increase manpower, create and get documentation files and manufacturing drawings online. Through funding it would be able that people dedicate their work to Pico Cross Flow, which accelerates the overall projects output, create more visibility and attract more people. Secondly it is important for long-time testing at a "pilot site". Initial funding and sufficient financial flexibility is a crucial factor for the further existence and success of the open source hydropower idea.

\section{D) Long-term Testing}

By having the turbine successfully tested in an artificial laboratory environment it is important to experience the turbines performance in "real conditions". Long term testing in "real working conditions" provides another perspective on stability and performance. This can be done at a "pilot-site", where the turbine can be tested in a timeframe of several months in real use cases. A necessary precondition for this is the availability of the electrical parts of the hydro power scheme. A generator and a governing system are inevitable. In regards to the electrical side of the hydro power scheme, preliminary considerations have been made and several options arose. None of them are possible without financial support.

In conclusion, to perform long term testing it is necessary to gain financial support and to find a convenient site to do so.

\section{Main Challenges and Outlook:}

Due to the fact, that hydro power is a complex technology depending on natural resources, it seems very difficult to get people contributing to the Pico Cross Flow community. Hydro power is a very small niche. People involved in hydro power are faced with electrical and 
mechanical engineering. Further, people who have adequate natural resources are not necessary the same people who are capable technicians and interested in open source hardware. Because of the complex technology, the dependence on the specific natural site conditions and the beneficiary, mainly living in the Global South, it seems very difficult to get people attracted to the project idea. As a consequence, a more specific and task orientated contribution is desirable and a serious challenge for the project development.

Another challenge in terms of money became visible. Unfortunately the financial benefit of an open source turbine for pico hydro power projects is very limited, since the financial benefit is only a small percentage of the overall hydro power project costs. As a consequence a goal might be to provide the complete electromechanical equipment as a "do it yourself kit" in an open source licensed form. By doing so the costs would be lower but the problem remains the same. The project costs could only be lowered to a specific percentage (depending on the natural conditions of the site) of the overall project costs resulting that the financial benefit is only a small percentage of the overall project costs.

Open source hydro power, seen from a economical perspective, is not very beneficial. The benefit is rooted in the decentralized and environmental friendly technology and the empowerment of people, strong related to the knowledge transfer.

An open source hydro turbine could be built easily in a workshop or in almost every hackerspace in the Global North. The challenge is that it is not much "needed" in the Global North. In New York, Paris or Tokyo people build laser cutters, drones or robots. Maybe it takes more time in rural electrification than in other areas where open source hardware is developed more dynamically. With these designed machines people connect more easily and use them in their free time and in form of a hobby. This is not the case in hydro power. Very 
little people do hydro power as their hobby. This is already rooted in the availability of natural sites and the legislation of land use. It is very difficult to find people who are able to implement a hydro turbine in their creek behind the house and at the same time have the interest in the technology as well as the financial capability to do so. The case is totally different for drones or laser cutters, for example. These machines are not dependent on specific natural site conditions or legal regulations of water use. It seems a real challenge for the project to open source practitioners and hydro power beneficiaries together.

Facing all these challenges, I still believe that the Pico Cross Flow turbine will be applied and implemented by a broader range of people when there is a broader acceptance of open source hardware in society. 


\section{Conclusion}

It is very important, if not crucial for next generations, to change our way of living. In the assessment of the global crisis it is very visible that increasing waste, dying of species and climate change is changing the living conditions on this planet. The growth and consumption based lifestyle does not lead to happiness and implies a server and irreversible damage on the environment. The ideas of open source might support a transformation away from growth and competition based lifestyle towards a more collaborative society.

Furthermore the dissertation elaborates on energy consumption and distribution, resulting a severe imbalance of access to electrical energy. Especially in rural areas in the Global South, access to electricity is very limited. Energy is a basic need and changes peoples lives essentially. For that reasons the practical part of the dissertation covers technical solutions for rural electrification and elaborates on its feasibility from the perspective of open source hardware.

The development of a technology comparison matrix helps to elaborate and highlight that renewable energy supply with hydro power seems the most appropriate for an open source approach. In a second step, the SWOT analysis investigates the most promising turbine type, resulting in a cross flow turbine design.

The hypothesis of this doctoral research is proven, such that it is visible that open source hardware can be an instrument of empowerment and growth for people and societies, surpassing capitalistic models, fostering collaboration, openness and development from a perspective of solidarity and sustainability. The practical and technical application in the field 
of renewable energy (clean, sustainable and autonomous) -as is the case of the Pico Cross Flow turbine that is designed and developed in this doctoral research- can provide a solution of great value, of great (re)applicability to a large number of people and communities.

The practical application of an open source hydro turbine for rural electrification could be seen as technically possible. At the same time it seems that this specific product is very hard to implement and get momentum to it. There are multiple causes for this. On one hand it is a product in a very special niche. On the other hand it is touching on very complex technology and at the same time so far only helping to save money on a fraction of the overall project costs. This means that the idea in general is feasible and also applicable. But at the same time it might not be the time for this change, since open source hardware is not as developed as open source software is today. It seems that open source hardware needs another couple of years to arrive in society in a broader range. When this is the case the probability is higher, that different NGOs, working in development work, apply and use open source technology instead of patented turbine designs.

It seems open source hardware is spreading slowly but constantly in various niche fields and potential markets, where the financial difference between the traditional and the open source solutions is huge. However, the process is very slow, especially in fields like rural electrification, where the market is very limited and the financial benefit of open source solutions is marginal.

Nevertheless, the idea of open source is an important contribution to a more sustainable way of economic development and will probably have a bigger influence in many fields in the future. 


\section{List of References}

Adafruit Industries. (n.d.). Home. Retrieved March 14, 2012 from http://www.adafruit.com

Ahmed, K. (1994). Renewable Energy Technologies - A Review of the Status and Costs of Selected Technologies. Washington, D.C.: The World Bank Group

Alliance for Rural Electrification. (2010). Energy access in the World. Retrieved February 6, 2012, from http://www.ruralelec.org/358.0.html?\&L=\%2525255C

------- (2010). What Electricity is capable of. Retrieved July 23, 2015, from http://www.ruralelec.org/18.0.html

------- (2010). Why renewable energy sources for rural electrification? Retrieved February 6, 2012, from http://www.ruralelec.org/17.0.html

------- (2010). Renewable Energy Technologies for Rural Areas. Retrieved July 18, 2015, from http://www.ruralelec.org/8.0.html?\&L=\%25255

------- (2011). Rural Electrification with Renewable Energy - Technologies, quality standards and business models. Brussels, Belgium: Renewable Energy House

Alward, R., Eisenbart S., Volkman J,. (n.d.). Micro-Hydro Power: Reviewing an Old Concept, AT Microfiche Reference Library / National Center of Appropriate Technology, Butte, USA

Aronson, D. (1996). Overview of Systems Thinking. Retrieved May 12, 2016, from http://www.thinking.net/Systems_Thinking/OverviewSTarticle.pdf

Arter, A. \& Meier, U. (1990). Hydraulics Engineering Maual - Harnessing Water Power on a Small Scale, Vol. 2. SKAT, Swiss Center for Appropriate Technology, St. Gallen Switzerland

Ashby, W., R. (1956). An Introduction to Cybernetics. John Wiley \& sons INC: New York

Bates, E. (2007). Biogas. Retrieved from the Intermediate Technology Development Group website: http://answers.practicalaction.org/our-resources/item/biogas, July 22, 2015

Bateson, N. (2011). An Ecology of Mind. Documentary, 60 min. Bullfrog Films.

Bauens, M. (2008). Peer to Peer, Retrieved October 6, 2011 from http://www.youtube.com/ watch?v=yY-lx7geHZM\&feature=related

Benkler, Y. (2005). Open Source Economics (TED talk). Retrieved March 14, 2012 from http://blog.ted.com/2008/04/16/yochai_benkler_1 
- (2006). The Wealth of Networks. How social Production Transforms Markets and Freedom. Yale University Press

Bertalanffy, v.L. (1968). General Systems Theory. New York: George Brallizer Inc.

Beutz, L., M. (2006). Networked Activism. Havard Human Rights Journal / Vol. 22

BiogasT (2012). Biogas Support for Tanzania. Retrieved from the Engineers without Borders website: http://www.ingenieure-ohne-grenzen.org/de/Regionalgruppen/Berlin/ Projekte/Biogas-support-for-Tanzania-BiogasT, retrieved March 302012

Blatter, I. (2016). Electrical schematic Drawing of a Wind Turbine. Drawing for Global Anchor e.V.

Bohm, D. (1980). Wholeness and the Implicate Order. Routledge, Reissue edition 2002

Boyle, G. (2004). Renewable Energy - Power for a sustainable future, Oxford: Oxford University Press

Brewer, J. (2016). Connecting the Dots 5: What If It's All Connected? Humanity And The Global Crisis? Retrieved on April 24, 2016 from https://blog.p2pfoundation.net/connecting-dots-5-connected-humanity-globalcrisis/2016/04/11

Briggs J. \& Peat F. D. (2000). Seven lessons of Chaos, New York: Harper Perennial 2000

British Hydropower Association (2005). A Guide to UK Mini-Hydro Developments v3.0, Retrieved from http://www.british-hydro.org/Useful_Information/A\%20Guide\%20 to\%20UK\%20mini-hydro\%20development\%20v3.pdf, August 20, 2015

Brown, J. (2012). What's a MakerBot Thing-O-Matic good for? Retrieved August 9, 2016 from http://www.macleans.ca/society/technology/whats-a-makerbot-thing-o-maticgood-for/

Brückmann, P. (2007). Autonome Stromversorgung. Staufen bei Freiburg, Oekobuchverlag

BuiltWith (n.d.). CMS Usage Statistics. Retrieved October 8, 2011 from http://trends.builtwith.com/cms

Bundesamt fuer Kulturfragen - Impulsprogramm PACER(1995). Kleinwasserkraftwerke Wahl, Dimensionierung und Abnahme einer Kleinturbine. Bern, Switzerland. Bundesamt fuer Kulturfragen

Business Strategies. (n.d.). What is a SWOT analysis? Retrieved March 10, 2012 from: http://www.bizstrategies.biz/swot-analysis.html

Beutz, L., M. (2006). Networked Activism. Havard Human Right Journal / Vol.22

Capra, B. (1990) (Director), Mindwalk. Overseas Filmgroup and The Atlas Production Company 
Capra, F. (1982). The Turning Point-Science, Society and the Rising Culture. London: Fontana Paperbacks (1996). The Web of Life - A new Synthesis of Mind and Matter. London: Harper Collins Publishers

------- (2002). The hidden Connections - A Science for Sustainable Living. New York: Anchor Books

------- (2015). Capra Course Trailer. Retrieved April 24, 2016 from http://www.capracourse.net/

Carbide 3D. (n.d.). Home. Retrieved August 16, 2016 from http://carbide3d.com/shapeoko/

Chapallaz, J.-M., Eichenberger, P., Fischer, G. (1992). MHPG Series - Harnessing Water Power on a Small Scale - Volume 11- Manual on Pumps used as Turnines.

St. Gallen: Swiss Center for Appropriate Technology (SKAT) and German Appropriate Technology Exchange (GATE), Braunschweig: Vieweg

Colorado Energy Office (n.d.). Colorado Small Hydropower Handbook. Retrieved from https://www.colorado.gov/pacific/energyoffice/resources-7, August 19, 2015

------- What are Fossil Fuels? Retrieved from http://www.conserve-energy-future.com/Disadvantages_FossilFuels.php, April 15 2016

Conserve Energy Future. (n.d.). Environmental Problems Retrieved from http://www.conserve-energy-future.com/15-current-environmental-problems.php, April 202016

Dalai Lama. (2005). The Universe in a Single Atom - The Convergence of Science and Spirituality, New York: Morgan Road Books

Dávila, C. (2010). Turbinas Hidráulicas, Presentation in the frame of hidroelectric cpacity building (Curso de capacitación en pico-nano hidro), November 2, 2010 in Teguscigalpa, Honduras

Deforestation. (2015). Retrieved April 21, 2016, from: http://environment.national geographic.com/environment/global-warming/deforestation-overview/

Dietrich, W. (2008). Variationen ueber die vielen Frieden - Band 1: Deutungen. Wiesbaden: VS Verlag fuer Sozialwissenschaften

------- (2010). Elicitive Conflict Transformation. Lecture at the University of Innsbruck, Austria

Edenhofer, O. (2011). Keynote Speaker at the Conference: "Micro Perspectives for Decentralized Energy Supply” April 7, 2012 Berlin

Einstein, A. (1950). Letter from 1950. Retrieved September 20, 2016 from http://www.onbeing.org/blog/einstein-sleuthing/3637 
Eisenring, M. (1991). MHPG Series - Harnessing Water Power on a Small Scale - Volume 9 Micro Pelton Turbines. St. Gallen: Swiss Center for Appropriate Technology (SKAT) and German Appropriate Technology Exchange (GATE)

Encyclopedia Britannica (2015). Daniel Bernoulli. Retrieved October 2, 2015, from http://www.britannica.com/biography/Daniel-Bernoulli

------- (2015). Hidrodynamica. Retrieved October 2, 2015, from http://www.britannica.com/topic/Hydrodynamica

EnDev (2015). Retrieved August 25, 2015 from http://www.endev.info

Energy Sector Management Assistance Program (2005). Stimulating the Picohydropower Market for Low-Income Households in Ecuador (Technical Paper 090). Washington D.C.: The World Bank Group

ENTEC (2009). DESIGN - Public Domain CrossFlow Turbine TP100c, Production Drawing, ENTEC Indonesia

Escobar, A. (1995). Encountering Development - The making and unmaking of the Third World, Princton University Press

European Small Hydropower Association, (1998). Laymans Guidebook - On how to develop a small hydro site. Brussels, European Commisssion

------- (2004). Guide on How to Develop a Small Hydrpower Plant. Brussels, European Commisssion

Fechner, C. A. (Director). (2010). Die 4. Revolution - Energy Autonomy. [DVD]. Germany: Fechner Media

Fein, P. (2011). Personal Conversation at the UN University for Peace in Costa Rica, December 2011

Focus (2011). 50 Places Linux is running you might not expect. Retrieved October 8, 2011 From http://www.focus.com/fyi/50-places-linux-running-you-might-not-expect/

Foundation for Deep Ecology (n.d.). Our Mission. Retrieved May 20, 2016 from http://www.deepecology.org/mission.htm

Foundation for Water \& Energy Education (2015). How the Hydraulic Cycle Works.

Retrieved July 27, 2015, from http://fwee.org/environment/what-makes-the-columbiariver-basin-unique-and-how-we-benefit/how-the-hydrologic-cycle-works

Fromm, E. (1976). To have of to be? New York: Continuum

Gabriel, S. (2007). Biodiversity 'fundamental' to economics. Retrieved April 22, 2016, from: http://news.bbc.co.uk/2/hi/science/nature/6432217.stm 
Gibb, A., Mota, C., (2015). Building Open Source Hardware - DIY Manufacturing for Hackers and Makers, Addison Wesley, Boston

Gilstrap, D. (2005). - Strange Attractors and Human Internaction: Leading Complex Organizations through the Use of Metaphors, Complicity: An International Journal of Complexity and Education, Volume 2 (2005), Number 1

GitHub (2011). Social Coding. Retrieved October 9, 2011 from http://www.github.com

Global Anchor (n.d.). About Us. Retrieved July 20, 2013, from http://www.globalanchor.org

Gnecco, M. Presentation in the frame of hidroelectric cpacity building (Curso Bombas como turbinas), March 18, 2012 in San Pedro Sula, Honduras

(2010). Training on pico cross flow. Manica Mosambique

------- (2012). Photo of Michell Banki Turbine fabrication in Manica, Mosambique

GNU Operating System (2010). The Free Software Definition, Retrieved October 8, 2011 from http://www.gnu.org/philosophy/free-sw.html

GoSol (2015). About. Retrieved July 27, 2015, from http://www.gosol.org/About (2015). Free the Sun Campaign. Retrieved July 27, 2015, from http://www.gosol.org (2015). Products. Retrieved July 27, 2015, from http://www.gosol.org/pruducts

------- (2015). University Pakistan. Retrieved July 27, 2015, from http://www.gosol.org/ Hybrid-Power-Generation-at-Air-University

Green Facts. (n.d.). Biodiversity \& Human Well Being. Retrieved April 22, 2016, from: http://www.greenfacts.org/en/biodiversity/

Harding, S. (n.d.). What is Deep Ecology? Retrieved May 24, 2016 from: https://www.schumachercollege.org.uk/learning-resources/what-is-deep-ecology

Hars, A. \& Ou, S. (2001). Working for Free? Motivations of Participating in Open Source Projects, Retrieved September 1, 2016 from https://www.computer.org/csdl/ proceedings/hicss/2001/0981/07/09817014.pdf

Hartvigsen Hydro (n.d.). Turgo runners. Retrieved April 24, 2015 from http://h-hydro.com/New_Site/turgo-runners/

Harvey, A. (1993). Micro Hydro Design Manual - A guide to small-scale water power systems, London: Intermediate Technology Publication

Hernandez, I. (2012). Configuration of Wind Turbines. Drawing for Global Anchor e.V.

Hogan, M. (2014). Water Pollution. Retrieved from Encyclopedia of Earth Website: http://www.eoearth.org/view/article/156920/ 
Heisenberg, W. (1962). Physics and Philosophy: The Revolution in Modern Science. New York: Harper \& Row Publishers

Hennicke, P. \& Fischedick, M. (2007). Erneuerbar Energien. München: Verlag C. H. Beck

Hobbes, T. (1651). Leviathan. Republished by the 'Gutenberg Project' 2003. Retrieved May 08, 2016 from: http://www.gutenberg.org/ebooks/3207

Holland, R. (1983). Micro Hydro Electric Power. Intermediate Technology Development Group (ITDG), London

Huffington Post, (2013). China Smog Photos Show How Bad Its Air Pollution Problem Has Become. Retrieved April 21, 2016, from http://www.huffingtonpost.com/2013/10/21/china-smog-photospollution_n_4137675.html

Hydroelectric Power. (n.d.). Battery and Energy Technologies. Retrieved June 17, 2014, from http://www.mpoweruk.com/hydro_power.htm

Inequality. (2015). Global Inequality. Retrieved 22 April, 2016 from http://inequality.org/global-inequality/

International Energy Agency (2010). Energy Poverty - How to make modern energy access universal? Paris, International Energy Agency

------- (2013). RENEWABLE ENERGY Medium-Term Market Report 2013. Paris, International Energy Agency

(2014). Key World Energy STATISTICS 2014, Retrieved

July 14, 2015, from https://www.iea.org/publications/freepublications/ publication/key-world-energy-statistics-2014.html

(2014). World Energy Outlook 2014 Electricity Database, Retrieved July 15, 2015, from http://www.worldenergyoutlook.org/resources/ energydevelopment/energyaccessdatabase/

Inversin, A. R. (1986). Micro Hydropower Sourcebook - A practical guide to design and implementation in developing countries. Washington D.C.: NRECA International Foundation

Jakubowski, M. (2011). Open Sourced Blueprints for Civilization. TED talks, Retrieved March 12, 2014 from http://www.ted.com/talks/marcin_jakubowski.html

Kaltschmitt, M. \& Wiese, A. (1997). Erneuerbare Energien - Systemtechnik, Wirtschaftlichkeit, Umweltaspekte. Berlin Heidelberg: Verlag Springer

Keller, D., R. (2009). Deep Ecology. Published in 'The Encyclopedia of Environmental Ethics and Philosophy - Volume 1' MACMILLAN REFERENCE USA, A part of Gale, Cengage Learning 
Kioskea (n.d.) Windows Family Tree. Retrieved May 14, 2012 from http://static. commentcamarche.net/en.kioskea.net/pictures/win-images-winhistorique.png

Koppensteiner, N. (2010). Transrational Interpretations of Peace. Lecture at the University of Innsbruck, Austria

Kuhn, S. T. (1962). The Structure of Scientific Revolutions, London, The University of Chicago Press, Ltd

Lasersaur. (n.d.). Home. Retrieved March 14, 2012 from http://labs.nortd.com/lasersaur

MacKillop, A. (1983). Hydropower. Wadebright Ecological Center, Wadebright UK

Make Zine. (n.d.). Technology on Your Time. Retrieved March 28, 2012 from

http://www.makezine.com/about

Malik, A. (2016). Ermittlung der Leistungswerte einer kleinen Durchstroemturbine. TU Wien

Maren, M. (1997). The Road to Hell - The Ravaging effects of Foreign Aid and International Charity, New York, The Free Press

Martí, J. (1974). Discursos. Habana Cuba, Editorial de Sciencias Sociales, Instituto Cubano del Libro

Matthias, H-B. (2003). Grundzuege der hydraulischen Maschinen und Anlagen. Wien, Technical University Vienna

MAVUNO (2013). About us. Retrieved from the MANUVO Website: www.mavuno.net, April 14, 2013

Mayr, F. (2012). Consciousising Relatedness. Saarbruecken, Akademikerverlag

McLuhan M. (1965). as quoted in Paradigms Lost: Learning from Environmental Mistakes, Mishaps and Misdeeds (2005) by Daniel A. Vallero, p. 367

Meadows, D.L., Meadows, D.H., Randers, J., \& Behrens, W. W. (1972). The Limits to Growth. New York, Universe Books

Merchant, C. (1980). The Death of Nature. New York, Harper \& Row.

Merriam Webster. (n.d.). Paradigm. Retrieved May 04, 2016 from: http://www.merriamwebster.com/dictionary/paradigm

Microhydropower (2015). The Firefly Micro Hydro System. Retrieved from the Microhydropower Website: http://www.microhydropower.net/ $\mathrm{mhp}$ group/portegijs/firefly_bm/ffbm_4_1-2.html, October 1, 2015

(2015). Retrieved on August 12, 2015, from: http://www.micro-hydropower.com/Pico-Hydro-Generator-System-brand-new.htm 
Mikulecky, D. C., (n.d.). Definition of Complexity. Retrieved May 08, 2016 from: http://www.people.vcu.edu/ mikuleck/ON\%20COMPLEXITY.html

Mills, A. (2016). Making a Difference with Open Source Science Equipment. Retrieved September 18, 2016 from http://www.mtu.edu/news/stories/2015/september/makingdifference-open-source-science-equipment.html

Mininchinelli, M. (2011). Personal Conversation at the Open Knowledge Conference in Berlin, July 1, 2011

Mockmore C. A. \& Merryfield F. (1949). The Banki Water Turbine. Bulleting Series No.25, Oregon State College

Moore, C. J., (2014). Choking the Oceans with Plastic. Retrieved from the New York Times website http://www.nytimes.com/2014/08/26/opinion/choking-the-oceans-withplastic.html?_r=0, April 212016

Mosonyi, E. (1987). Water Power Development - Volume One - Low Head Power Plants, Budapest: Akadémiai Kiadó

Naess, A. (1973). The Shallow and the Deep, Long-Range Ecology Movement: A Summary. Retrieved on May 24, 2016 from https:/www.researchgate.net/ publication/233029642_The_Shallow_and_the_Deep_LongRange_Ecology_Movement_A_Summary

Netcraft (2016). Web server developers: Market share of active sites. Retrieved April 16, 2016 from http://news.netcraft.com/archives/2016/03/18/ march-2016-web-server-survey.html

Netmarketshare (2011), Desktop Browser Market Share. Retrieved November 02, 2011 from: https:/www.netmarketshare.com/browser-marketshare. aspx?qprid $=0 \&$ qpcustomd $=0$

(2016a), Desktop Operating Systems Market Share. Retrieved August 08, 2016 from: https://www.netmarketshare.com/operating-system-marketshare.aspx?qprid $=10 \& q$ pcustomd $=0$

------- (2016b), Mobile / Tablet Operating Systems Market Share. Retrieved August 08, 2016 from: https://www.netmarketshare.com/operating-system-marketshare.aspx? qprid $=8 \&$ qpcustomd $=1$

NixCraft (n.d.) Linux Timeline. Retrieved May 14, 2012 from http://www.files. cyberciti.biz/uploads/tips/2007/06/44218-linuxdistrotimeline-7.2.png

Noble, N. (2008). Energy From the Wind. Retrieved from the Intermediate Technology Development Group website: http://answers.practicalaction.org/ourresources/item/energy-from-the-wind, July 22, 2015 
Oakley, E.G. (1995). A Brief History of Psychology. Retrieved May 10, 2016 from: http://www.psych.utah.edu/gordon/Classes/Psy4905Docs/ PsychHistory/index.html\#maptop

Open Hardware and Design Alliance (OHANDA), (n.d.) About. Retrieved March 14, 2012 from http://www.ohanda.org

Open it Agency. (n.d.). Open Source. Retrieved August 10, 2016 from: http://openitagency.eu/open-source/

Open Relief. (n.d.). Welcome to open Relief. Retrieved August 15, 2016 from: http://www.openrelief.org/

Open Source Beehives. (n.d.). What is the Open Source Beehives Sensor? Retrieved August 11, 2016 from: http://docs.opensourcebeehives.net/docs/alpha-sensor-kit

Open Source Ecology. (n.d.). Global Village Construction Set. Retrieved March 12, 2012 from http://www.opensourceecology.org/

------- (2011). The Open Source Ecology Paradigm. Retrieved April 2, 2016 from: http://opensourceecology.org/wiki/Open_Source_Ecology_Paradigm

------- (2016a). Tractor. Retrieved August 16, 2016 from http://opensourceecology.org/portfolio/tractor/

(2016b). BFI Challenge \& Global Village Construction Set in 2 Minutes. Retrieved August 16, 2016 from http://opensourceecology.org/global-village-construction-setgvcs-in-2-minutes/

Open Source Hardware Association (OSHWA), About. Retrieved August 8, 2016 from http://www.oshwa.org/about/

-------- Best Practices for Open Source Hardware 1.0. Retrieved October 10, 2016 from http://www.oshwa.org/sharing-best-practices/

Definition. Retrieved August 30, 2016 from http://www.oshwa.org/definition/

-------- Open-Source Hardware FAQ. Retrieved

August 8, 2016 from http://www.oshwa.org/faq/

Open Source Initiative (OSI), The Open Source Definition. Retrieved October 8, 2011 from http://www.opensource.org/docs/osd

Open Source List (n.d.). Open Source Software for Windows, Linux, Mac OSX. Retrieved April 1, 2012 from http://www.opensourcelist.org/

Overton, R. (2007). Feasibility Studies Made Simple, Martin Books, Australia

Punter, A. (2011). Solar Thermal Energy. Retrieved July 24, 2015 from the Intermediate Technology Development Group website: http://answers.practicalaction.org/ourresources/item/solar-thermal-energy 
Pangaro, P. (2012). What is cybernetics? Movie retrieved May 12, 2016 from http://www.pangaro.com/definition-cybernetics.html

Population (2015). Retrieved July 14, 2015, from http://www.worldometers.info/world -population/

P2P Foundation (n.d.). Open Source Hardware. Retrieved August 10, 2016 from https://wiki.p2pfoundation.net/Open_Source_Hardware

Raabe, J. (1985). Hydro Power - The Design, Use, and Function of Hydromechanical, Hydraulic and Electrical Equipment. Düsseldorf: VDI-Verlag

Renewables First (2015). Retrieved September 14, 2015 from: https://www.renewablesfirst.co.uk/hydropower/hydropower-learning-centre/peltonand-turgo-turbines/

Repairable Machines. (n.d.). About. Retrieved April 2, 2012 from http://www.repairablemachines.com/about.html

Rheingold, H. (2005). The new power of collaboration. Retrieved September 1, 2016 from https://www.ted.com/talks/howard_rheingold_on_collaboration?language=en

Rifkin, J. (1980). Entropy - A New World View, New York: The Viking Press

(2011). The Third Industrial Revolution - How lateral power is transforming energy, the economy, and the world. New York: PALGRAVE MACMILLAN

------- (2014). The Zero Marginal Cost Society - The Internet of Things, The Collaborative Commons, and the Eclipse of Capitalism. New York: PALGRAVE MACMILLAN

Robert, A. (2011). Introduction to the Systemic Approach. Lecture at the United Nations Mandated University for Peace, UPEACA, El Rodeo, Costa Rica

Rodriguez, F. (2015). Picture of Cross-Flow turbine for Ocotal, Nicaragua. Retrieved via Skype after personal conversation, January 2015

Scharmer, C. O. (2009). Theory U-Leading from the Future as It Emerges, San Franscisco: Berret-Koehler Publishers Inc.

Scholarpedia (2008). Self Organization. Retrieved October 9, 2011 from http://www.scholarpedia.org/article/Self-organization

Schuhmacher, E. F. (1973). Small is Beautiful - A Study of Economics as if Peopled Mattered. London, Vintage (published 2011)

Shirky, C. (2009). Here Comes Everybody: The Power of Organizing Without Organizations. New York: Penguin Books.

Shuttleworth Foundation. (n.d.). Urban snap-fit garden kits. Retrieved August 20, 2016 from https://shuttleworthfoundation.org/fellows/aaron-makaruk 
Singularily Hub. (n.d.). Science, Technology. The Future of Mankind. Retrieved March 12, 2012 from http://singularityhub.com/2010/05/10/13-open-source-hardwarecompanies-making-1-million-or-more-video/

Smith, L. (2015). 10 Species dying out in the Wild. Retrieved April 21, 2015 from: http://www.ibtimes.co.uk/endangered-species-day-2015-10 -species-dying-out-wild-1501096

Social Media Workgroup (2011). Visualizing the commit history of the open source Apache webserver project with code_swarm. Retrieved October 9, 2011 from http://socialmedia.hpc.unm.edu/?p=540

Stallman, R. (2007). Can You Trust Your Computer? - gnu.org, Retrieved September 1, 2011, from http://www.gnu.org/philosophy/can-you-trust.html

(2011a). Proprietary Software keeps Users Helpless - Tech Radar, Retrieved October 8, 2011, from http://www.techradar.com/news/software/-proprietarysoftware-keeps-users-helpless--963248

------- (2011b). Personal Conversation at the Open Knowledge Conference (OKCon) July 1, 2011 in Berlin

Steiner, R. (2003). Grün gewinnt - Die letzte Ölkrise und danach. Zürich: Verlag Orell Füssil

Strathclyde University. (n.d.). Photovoltaics. Retrieved April 2, 2012 from http://www.esru.strath.ac.uk/EandE/Web_sites/01-02/RE_info/photovoltaics.htm

The Rules. (2016). Inequality Video Fact Sheet. Retrieved April 25, 2016 from http://therules.org/inequality-video-fact-sheet/

Thomson, C. C. \& Jakubowski, M. (2012). Toward an Open Source Civilization. Article published in innovations (A quarterly journal published by MIT press), volume 7, issue 3

Threat Deforestation. (2015). Retrieved April 22, 2016 from the WWF website: https://www.worldwildlife.org/threats/deforestation

Timelab. (n.d.). About. Retrieved March 24, 2012 from http://www.timelab.org/en/info/en/about

Tiemersma, J. J., \& Heeren N. A., (n.d.). Small Scale Hydropower Technologies, AT Microfiche Reference Library / TOOL foundation, Amsterdam \& Center for Appropriate Technology, Delft

Tipler, P. (1987).College Physics. New York: Worth

Turgo Runners. (2015). Retrieved from Hartvigsen Hydro's Website, September 22, 2015, http://h-hydro.com/New_Site/turgo-runners/ 
Union of Electricity Industry (2003). Efficiency in Electricity Generation. Brussels: EURELECTRIC

United Nations Development Program (2009). The Energy Situation in Developing Countries. New York, UNDP

United Nations - Population Division (2004). World Population to 2300. New York, United Nations

------- (2012). World Population 2012 Wall chart,

Retrieved July 15, 2015, from http://www.un.org/en/development/desa/population/ publications/trends/wpp2012.shtml

University of Kansas (2015). SWOT Analysis: Strenghts, Weaknesses, Opportunities and Threats. Retrieved July 30, 2015, from: http://ctb.ku.edu/en/table-of-contents/ assessment/assessing-community-needs-and-resources/swot-analysis/main

Vöstalpine Böhler Welding Group GmbH, (n.d.). Hydro Power, Retrieved from http://www.utp-maintenance.com/Industries/Power-Generation/Hydro-power, September 25, 2015

Watson, D. (2010). Wind Turbines and the Energy in Wind. Retrieved July 24, 2015, from http://www.ftexploring.com/energy/wind-enrgy.html

Watter, H. (2011). Regenerative Energiesysteme - Grundlagen Systemtechnik und Anwendungsbeispiele aus der Praxis. Wiesbaden: Vieweg+Teubner Verlag

WebmasterPro (n.d.). Webanalyse - Open Office auf ueber 21\% der Computer. Retrieved October 8, 2011 from http://www.webmasterpro.de/portal/news/2010/01/25/ verbreitung-von-office- programmen-openoffice-ueber-21.html

Wiener, N. (1948) Cybernetics - or, Control and Communication in the Animal and the Machine. Cambridge: Massachusetts Institute of Technology

Williams, A. (1995). Pumps as Turbines - A user's guide. London: Intermediate Technology Publications

Wissenz, E. (2015). Energy Problems. Retrieved April 15, 2015, from http://www.decent-democracy.org/Energy-Problems.html

WISIONS (2010). Access to Electricity - Technological options for community-based solutions. Wuppertal: Wuppertal Institute for Climate, Environment and Energy

Woodbank Communications (2005). Hydroelectric Power, Retrieved July 28, 2015, from http://www.mpoweruk.com/hydro_power.htm

World Bank, (2016). GDP Ranking. Retrieved April 22, 2016 from http://data.worldbank.org/data-catalog/GDP-ranking-table 
World Wide Fund for Nature (WWF). (2014). Living Planet Report-Species and spaces, people and places. Gland, Switzerland. WWF

(2015). Living Blue Planet Report-Species, habitats and human well-being. Gland, Switzerland. WWF

Wyllys, R. E. (2000). Overview of Open Source Movement, Retrieved March 13, 2012, from http://www.ischool.utexas.edu/ 138613dw/readings/OpenSourceOverview.html,

Zimmerman, M., E. (1989). Introduction of Deep Ecology. Retrieved May 24, 2016, from http://www.context.org/iclib/ic22/zimmrman/, published by the Context Institute

Zoetrope Photo Collage done with the following sources:

- Earthship Windturbine, Retrieved May 14, 2012 from http://earthship.com/Systems/vertical-axis-wind-power-generation-final-product.html

- Chispo Wind Generator, Retrieved May 14, 2012 from http://www.velacreations.com/chispito.html

- Zoetrope, Retrieved May 14, 2012 from http://www.earthtechling.com/2011/03/diywind-turbine-project-goes-open-source

- Hugh Piggots Wind Turbine, Retrieved May 14, 2012 from

http://www.otherpower.com/guemes04front.html 


\section{List of Physical Units}

\begin{tabular}{|c|c|c|}
\hline Acronym & Description & {$[\text { SI-Unit }]^{52}$} \\
\hline$\alpha$ & Absolute Velocity Angle & {$\left[{ }^{\circ}\right]$} \\
\hline$\beta$ & Relative Velocity Angle & {$\left[{ }^{\circ}\right]$} \\
\hline A & Rotor Area & {$\left[\mathrm{m}^{2}\right]$} \\
\hline $\mathrm{c}$ & (Wind) Speed / Absolute Speed of Water Jet & {$[\mathrm{m} / \mathrm{s}]$} \\
\hline$c_{\mathrm{p}}$ & Power Coefficient & {$[-]$} \\
\hline $\mathrm{D}$ & Rotor Diameter & {$[\mathrm{m}]$} \\
\hline $\mathrm{g}$ & Gravity (9.81) & {$\left[\mathrm{m} / \mathrm{s}^{2}\right]$} \\
\hline $\mathrm{H}$ & Height & {$[\mathrm{m}]$} \\
\hline $\mathrm{k}$ & Nozzle Coefficient $(0.967)$ & {$[-]$} \\
\hline $\mathrm{m}$ & Mass Flow Rate & {$[\mathrm{kg} / \mathrm{s}]$} \\
\hline $\mathrm{p}$ & Pressure & {$\left[\mathrm{kg} / \mathrm{m} \mathrm{s}^{2}\right]$} \\
\hline $\mathrm{P}$ & Power in Watts & {$[\mathrm{W}]$} \\
\hline Q & Flow of Water in Cubic Meters per Second & {$\left[\mathrm{m}^{3} / \mathrm{s}\right]$} \\
\hline $\mathrm{r}$ & Radius of Turbine Runner & {$[\mathrm{m}]$} \\
\hline $\mathrm{R}$ & Gas Constant & {$[\mathrm{J} / \mathrm{mol} \mathrm{K}]$} \\
\hline$\rho$ & Density & {$\left[\mathrm{kg} / \mathrm{m}^{3}\right]$} \\
\hline $\mathrm{n}$ & Revolutions per Minute & {$[\mathrm{rpm}]$} \\
\hline $\mathrm{T}$ & Temperature & {$[\mathrm{K}]$} \\
\hline toe & $\begin{array}{l}\text { Ton of oil equivalent (toe) is a physical unit equal to } \\
\text { amount of energy released by burning one ton of crude oil. } \\
\text { The amount of energy is approximately } 42 \text { Giga Joules }\end{array}$ & {$[\mathrm{J}]$} \\
\hline $\mathrm{u}$ & Turbine rotational Speed & {$[\mathrm{m} / \mathrm{s}]$} \\
\hline $\mathrm{V}$ & Volume Flow Rate & {$\left[\mathrm{m}^{3} / \mathrm{s}\right]$} \\
\hline $\mathrm{W}$ & Angular Speed & {$[\mathrm{m} / \mathrm{s}]$} \\
\hline$\eta$ & Efficiency & {$[-]$} \\
\hline
\end{tabular}

\footnotetext{
${ }^{52}$ SI (from the French Système International d'Unités) is the international system of units, published by the International Committee for Weights and Measures.
} 


\section{Resumen en Castellano}

Estamos viviendo en un sistema global basado en el crecimiento, donde el sistema económico nos está obligando a explotar la tierra y su naturaleza. Nos enfrentamos con desafíos medioambientales globales, como la deforestación, la contaminación del aire y del agua, la disminución de especies y los glaciares que se funden. Al mismo tiempo desarrollamos tecnologías aún más sofisticadas para la explotación, guerras salariales para conquistar y proteger los recursos e incrementar la producción de residuos sin ideas de cómo proceder en el futuro.

Nuestro modo de vida actual y nuestro sistema económico exigen cada vez más energía. Los combustibles fósiles y la energía nuclear parecen apoyar nuestro estilo de vida de consumo moderno y exigente de energía. Estos recursos energéticos están disponibles a través de una estructura jerárquica lineal y altamente centralizada, de arriba hacia abajo.

Los complejos sistemas industriales, tanto organizacionales como tecnológicos, son el principal motor de la destrucción ambiental global, la desigualdad socioeconómica y la insatisfacción social. Estamos vendiendo recursos de la Madre Tierra sin conciencia de sus efectos sobre el medio ambiente. Sin embargo, el crecimiento en un sistema finito necesariamente tiene que llegar a su fin. Aún más alarmante, las Naciones Unidas proyectan un crecimiento de la población mundial "de 6.100 millones en 2000 a 8.900 millones en 2050, aumentando un 47\%" (Naciones Unidas, 2004).

Todo esto tiene que ser visto en el marco de un paradigma cambiante, especialmente en un cambio de información y energía. Vivimos en una transición de energía desde una era de combustibles fósiles (carbón, petróleo y gas natural) hasta una era solar, impulsada por energía renovable del sol. Al mismo tiempo estamos viviendo una revolución en la tecnología 
de la comunicación que conecta a los seres humanos en este planeta como nunca antes. Estas transiciones ya tendrán un profundo impacto en nuestras vidas, sociedades y estructuras sociales. ¿Cómo afecta este cambio interdisciplinario, tal vez un cambio de conciencia, nuestros sistemas económicos y sociales? ¿Cómo nos organizamos y nos motivamos? Si el crecimiento material personal no es nuestra fuerza motriz, ¿qué es entonces?

Este proyecto de investigación explora el potencial transformador de la sociedad de las ideas “Open Source" (OS) (código abierto), especialmente en relación con el Open Source hardware (OSH). El enfoque de Open Source se investiga desde diferentes ángulos, y luego se aplica prácticamente en el campo de la electrificación de áreas rurales a través de una turbina de energía hidroeléctrica de código abierto.

\subsection{Preámbulo}

Cuando decidí de seguir una trayectoria académica en el campo de los "Estudios de Paz", luchaba profundamente con lo que percibía como una contradicción dentro de mí: Ser ingeniero profesional, creer en la belleza y la lógica de estructuras y mecanismos complejos, y al mismo tiempo sintiéndome profundamente conectado a la idea de paz y sus diversas manifestaciones. Cuando empecé el programa de Maestría en "Paz, Desarrollo, Seguridad y Transformación de Conflictos Internacionales" en la Universidad de Innsbruck, como parte de nuestra fase de introducción me tenía que presentar a mis nuevos colegas con una breve biografía de quién soy, lo que he hecho en el pasado y lo que me gustaría hacer en el futuro. Uno de mis compañeros de clase me respondió: "Usted no tiene que preocuparse por sus fondos inusuales de ingeniería y su trabajo en energía renovable. Al final, los estudios sobre la paz tienen que ver con la transformación de la energía. Tal vez te va a gustar". Me tomó 
algún tiempo, pero finalmente me di cuenta de la verdadera esencial que está en esa declaración. Esta esencia me llevó al programa de doctorado en Castellón, comprometido con esta investigación.

Supongo que todo se reduce a su intención. Crecí en una zona bastante rural, y desde que era niño siempre tenía una fuerte conexión con la naturaleza. Cuando estábamos jugando en el bosque, tallando bonitos palos de madera, siempre eran los otros niños los que tenían el palo más grande. Me recuerdo que me preguntó: ¿Vale la pena matar esta planta sólo porque quiero jugar con mi cuchillo? Sobre todo, prefería cortar una rama en lugar de decapitar todo el tronco de un pequeño árbol. Supongo que mi conciencia para el medio ambiente viene de ese tiempo. Siempre me interesaba la tecnología y quería saber cómo funcionan las cosas. Cuando era niño jugué con todo tipo de juguetes técnicos. Fue interesante porque había mucho que descubrir. Me recuerdo que cortó el cable de un coche que solamente funcionó con cable. Mi intención era hacer que fuera inalámbrica a control remoto, y en el proceso me di cuenta de que no funciona tan fácilmente. Cortar el cable no logra ese objetivo, e incluso peor, hacía que mi coche ya no funcionó.

Después de la escuela, he estudiado ingeniería mecánica y he entrado a una físicamatemáticas, camino científico-lineal de la educación y del pensamiento. He aprendido cómo funcionan las cosas técnicas, pero la cuestión de la intención se mantuvo, sobre todo después de mis estudios, cuando empecé a trabajar como ingeniero en energía renovable (gran energía hidroeléctrica). Desde un punto de vista técnico, fue emocionante, interesante y desafiante diseñar y llevar físicamente un corredor de turbina de 150 toneladas a un sitio en medio de la nada, en una sección natural y pura de un río donde no hay caminos. Pero también era consciente de que la máquina en ejecución, así como el proceso de construcción e 
implementación tenía graves impactos sobre el medio ambiente. Y me di cuenta de que la idea principal era a menudo obtener beneficios y satisfacer a los inversores, y no proporcionar electricidad a las personas necesitadas. Supongo que la intención de un empresario industrial no es necesariamente la "mejor" solución técnica o "mejor" sostenible, sino una solución técnica para el "máximo beneficio" de la empresa. Además, la competencia entre las empresas que persiguen el mismo objetivo es sorprendentemente problemática. En esos enormes proyectos, que gestionan millones de euros muchas empresas están involucradas. Todas estas empresas trabajan juntas, comparten responsabilidades y quieren construir la misma planta. Sin embargo, cada parte involucrada en un proyecto también busca su propia ventaja. El objetivo es esforzarse por obtener beneficios mediante la adhesión a la tarea específica de la empresa asignada y para ser legalmente seguro después de que el trabajo está terminado. La intención de co-crear y trabajar juntos se vuelve casi secundaria. La falta de motivación para co-crear ideas fue frustrante para mí como un ingeniero consciente.

Mi experiencia con el desarrollo de software de código abierto me ayudó en darme cuenta de que el enfoque descrito anteriormente apunta en una dirección problemática e insatisfactoria. Estoy convencido de que el desarrollo de la tecnología en un entorno económico está impulsado en gran parte por una "intención errónea". En cierta medida, niega la idea central y los beneficios de los seres humanos trabajando juntos, y en segundo lugar, afecta el medio ambiente de una manera irresponsable. He empezado el desarrollo de software de código abierto como hobby. Estaba rodeado de personas con ideas afines en este ambiente. Personas interesadas en mejorar un producto debido a su propia experiencia con él. He sentido que la fuerza impulsora para el desarrollo y la mejora del producto era completamente diferente, alineada con la intención de uno. 
Como tema de investigación para completar el programa en estudios de paz, escogí la electrificación rural con energía renovable y comencé a trabajar en el campo de la electrificación rural a través de hidroeléctricas de escala pequeña en Nicaragua y Honduras. He facilitado y apoyado técnicamente el uso de la energía hidroeléctrica de escala pequeña, que en este caso tuvo muy poco impacto en el medio ambiente y benefició principalmente a las personas que carecían de acceso a la energía. Como parte de mi trabajo, me quedé con las comunidades, literalmente en medio de la nada para apoyar la instalación y acompañar el proceso de electrificación. Trabajar con personas que no están conectadas a la electricidad "electrificada" como me gusta decir - y en cambio viven en profunda conexión con la naturaleza, me ofreció la posibilidad única de investigar los efectos de la energía sobre los seres humanos y las estructuras sociales e investigar las relaciones entre la gente y la naturaleza. En estas zonas rurales y comunidades las necesidades técnicas específicas son visibles. Es evidente que muy poca "tecnología" es necesaria para satisfacer las necesidades de energía en las zonas rurales.

He desarrollado un enfoque técnico al tema, pero nunca confiaba plenamente en él. En el proceso de esta investigación asistí en dos conferencias que cambiaron mi perspectiva. Una conferencia se centró en el suministro descentralizado de energía, lo que me permitió desarrollar nuevas perspectivas en el campo del trabajo de desarrollo. Tenía la impresión de que muchas personas bien vestidas trataban de vender sus soluciones y productos a otras personas bien vestidas que les ofrecían algo de dinero. Parecía que este proceso no tenía mucho que ver con las personas necesitadas, aunque se mostraban en las imágenes de muchas diapositivas de su PowerPoint.

La otra conferencia sobre conocimientos abiertos casi me abrumó, dada la gente inspiradora, ideas y enfoques que encontré. La energía y el ambiente en esta conferencia fueron 
impresionantes y reflejaron muy bien el estado de desarrollo en este campo, donde muchas cosas pasan muy rápido en estos días. Este contexto agregó el elemento que faltaba y completó mi tema: combinar soluciones técnicas con el enfoque de código abierto.

Esto también me ofreció una avenida única para mezclar los dos campos en los que trabajaba a lo largo de los años, y toca fuertemente la intención de crear una solución técnica o un producto para la electrificación rural.

Tomando este fondo diverso en la consideración, mi investigación del $\mathrm{PhD}$ extiende en la factibilidad de las soluciones a pequeña escala de la energía hidráulica del Open Source. Debe ser visto como un estudio de viabilidad técnica y social, que consta de partes teóricas y prácticas. Mi motivación para elegir este tema es multidimensional. Por un lado, me interesa la energía y la electrificación rural. La salida de la pobreza energética y el acceso a la energía (renovable) representan una necesidad básica. Es técnicamente fascinante reducir los principios físicos de trabajo a su mínimo, que a menudo es más que suficiente para transformar pequeñas cantidades de energía. La electrificación rural en los países menos desarrollados es un tema particularmente intrigante y práctico que afecta la calidad de vida de muchas personas de una manera muy profunda. Por otro lado, es fascinante ver cómo el enfoque de código abierto está ofreciendo un nuevo modo de cómo las personas interactúan y crean bienes comunes a una escala global. Este enfoque se ha visto en el desarrollo de software y con Wikipedia, por ejemplo. Muchas personas prácticamente se reúnen, trabajan en un problema común y desarrollan una solución común, que puede ser utilizada por todos. Y esto también comienza a suceder con el hardware, donde los productos reales se desarrollan a escala global. El hardware de código abierto para la electrificación rural es la combinación de estos dos temas, creando un campo de investigación desafiante y 
multidisciplinario. Estoy muy contento de trabajar en este campo, combinando una perspectiva de ingeniería con una perspectiva social, de estudios de paz.

\subsection{Objetivo de la Investigación y Pregunta Central}

El tema de este estudio está relacionado con la electrificación, y especialmente, con la electrificación rural en áreas rurales los que se conoce como países de bajo ingreso económico. Por un lado, compara la aplicabilidad de diferentes soluciones técnicas de energía renovable, como bioenergía, energía eólica, energía solar concentrada, fotovoltaica e hidroeléctrica para la electrificación de áreas remotas. Por otro lado, ofrece un estudio de factibilidad que analiza el desarrollo y la implementación de dicha tecnología. Por lo tanto, una gran parte de esta investigación se centra en el concepto de hardware de código abierto y posibilidades de fabricación descentralizada, que adopta el enfoque de desarrollo de software de código abierto con el de hardware. Este enfoque puede ser visto como una forma postindustrial de economía con una intención no capitalista. Se refiere a un método de desarrollo más orgánico, auto organizado, colaborativo y tal vez más natural. Además, el enfoque está relacionado con la cuestión de la comunicación mediante nuevos medios y redes, lo cual es especialmente visible en el proceso de desarrollo del producto.

La pregunta de investigación concreta es si las soluciones de energía renovable a pequeña escala pueden ser desarrolladas e implementadas con un enfoque de código abierto y cuáles de las diferentes tecnologías contienen el potencial más prometedor. Del mismo modo, este estudio busca responder a las preguntas de cómo y dónde iniciar tal esfuerzo y también investiga y acompaña su realización práctica. 
Respondiendo a las preguntas anteriores, quiero elaborar la posibilidad de desarrollar soluciones de energía renovable que se basen en métodos de colaboración y de intercambio de tecnología sin ceder a la motivación financiera y sin correr el riesgo de imponer una determinada tecnología a otros. La motivación proviene del interior lo que significa que la inducción al cambio o al acto se basa en un deseo inherente. Esto se reduce a la intención. El código abierto parece ser el instrumento adecuado para llevar a cabo este enfoque, ya que uno de las ideas esenciales es la auto-organización y creatividad.

\section{Hipótesis:}

La hipótesis, que es la base de este estudio, puede ser enmarcada de la siguiente manera: ¿Cómo es el hardware de código abierto factible y cómo podría ser un modelo social, más allá de sociedades capitalistas, de crecimiento y competitivas, que fomente la colaboración, la apertura y el compartir? ¿Cómo contribuye una turbina hidroeléctrica pico a este enfoque? La hipótesis se aplica prácticamente y se investiga en el campo de la electrificación rural.

\section{Objetivo de la investigación:}

El objetivo de este proyecto doctoral es investigar cómo el enfoque de código abierto puede ser una alternativa a nuestro "estilo de vida de consumo" y cómo afecta las estructuras sociales y nuestra (re) conexión con la naturaleza. Diferentes valores como la conciencia, la intención y la motivación están en el centro de este enfoque. La cooperación global, el beneficio mutuo y la compasión en lugar de la competencia, la avaricia y el crecimiento serán centrales para una forma post-materialista de la sociedad.

Sin embargo, como ingeniero que trabaja en la electrificación de áreas remotas con energía renovable, una gran parte de la investigación se llevará a cabo en el contexto de la ciencia física. 
Este componente del proyecto, un estudio de factibilidad para hardware de código abierto y electrificación rural, desarrolla principios físicos y soluciones técnicas para la transformación de energías renovables. El objetivo final de este componente es construir las bases científicas para el desarrollo de un producto para la electrificación rural, que luego será orquestado en la última parte del estudio - la aplicación práctica y el diseño de una turbina hidroeléctrica. Esta última parte se centra en el diseño y la fabricación de una turbina hidroeléctrica pico desde la perspectiva de la OSH y puede ser vista como el "objetivo práctico"; una contribución al movimiento del código abierto en energía renovable.

\subsection{Metodología y Estructura de la Disertación}

Esta tesis doctoral consta de varias partes, comenzando con el contexto histórico, los desafíos globales y el período de transición en el que vivimos. Se describe un posible cambio de paradigma, un cambio social en la motivación y la intención. Existe una sección de investigación teórica sobre el consumo, las estructuras sociales y nuestra conexión con la naturaleza, basada principalmente en la investigación de la literatura clásica.

La parte técnica de la investigación se basa en el método de un estudio de factibilidad clásico. Un estudio de factibilidad es un enfoque escrito formalizado que evalúa el potencial y la viabilidad de un producto, proyecto o idea. Es una herramienta de gestión, utilizada como base para la toma de decisiones, utilizada principalmente para ideas empresariales y técnicas. Se reúne y compara hechos y cifras útiles, que ayudan a cubrir una pregunta desde diferentes perspectivas y ángulos. Este proceso debe considerar todas las áreas de una idea y asegurar que todos los posibles ángulos y facetas de la influencia potencial están cubiertos. Su objetivo es ayudar a identificar fortalezas y debilidades objetivamente y racionalmente verificables, así como oportunidades y amenazas. Ambos obstáculos y oportunidades se hacen visibles y 
también deben documentarse. El estudio de viabilidad realizado en esta investigación evaluará y analizará las siguientes preguntas:

○ ¿Cuáles son las necesidades y el potencial de la electrificación rural?

○ ¿Cuáles de las tecnologías propuestas son trasladables?

○ ¿Cuál es el marco legal?

○ ¿Ya se ha hecho una vez? ¿Afectan las patentes la idea?

○ ¿Hay otras personas potencialmente interesadas y cuáles son sus ideas?

¿ ¿Hay otras personas que podrían ser potenciales cooperadores?

¿ ¿Es técnicamente factible?

Estas preguntas se correlacionan con las principales categorías de un estudio de factibilidad clásico - las denominadas categorías TELOS (Overton, 2007: 6). Como tal, las preguntas se elaboran dentro de estas cinco categorías en sus respectivos subcapítulos:

○ Viabilidad tecnológica

○ Viabilidad económica

○ Viabilidad jurídica

- Factibilidad organizativa

○ Planificación de factibilidad

La viabilidad tecnológica abarca todos los aspectos de la viabilidad técnica de las soluciones de energía renovable a pequeña escala. Un estudio de factibilidad económica, también llamado análisis de costo / beneficio, es llevado a cabo por las empresas para averiguar si un producto es capaz de generar un beneficio esperado y mensurable. Una empresa tiene que sopesar los costos versus los beneficios antes de proceder de una manera exacta y precisa. En el marco de este estudio, la viabilidad económica no se cubrirá en profundidad ya que la obtención de ganancias no es el propósito o meta de los proyectos de código abierto. No obstante, la solución técnica se analizará en relación con los costes de la energía transferida. La viabilidad jurídica abarca la cuestión de si una solución técnica es incompatible con las 
soluciones técnicas ya existentes y las patentes de las empresas establecidas. La factibilidad operativa generalmente cubre si y cómo una organización es capaz de realizar una idea, incluyendo mano de obra, equipo y herramientas. Además, este aspecto sólo se abordará ligeramente en este estudio, ya que esta no es la forma organizativa de los proyectos de código abierto. La factibilidad de la programación cubre la línea de tiempo o la hoja de ruta de tal proyecto y su tiempo de desarrollo técnico. Este es un factor crucial en las empresas que buscan colocar sus productos en el mercado más rápido que sus competidores. Hay aspectos adicionales de la viabilidad de los planes, que afectan a la utilidad de un proyecto. Por ejemplo, si el proceso de desarrollo y realización de un proyecto, evidentemente, toma demasiado tiempo, podría perder su utilidad y fracasar. La viabilidad de la programación permite estimar y medir la razonabilidad de la línea de tiempo de un proyecto. Esto también se desarrollará más en las estrategias discutidas.

La evaluación de los hechos y cifras requeridos para responder a las preguntas planteadas anteriormente se hará a través de un análisis Fortalezas - Debilidades - Oportunidades Amenazas (FODA) (inglés: SWOT) basado en una matriz de análisis de viabilidad: "El análisis FODA es la base para desarrollar su estrategia y tácticas que luego se convierten en la hoja de ruta para escribir su plan operativo de negocio" (Business Strategies, 2012). Representará una tecnología previamente seleccionada $\mathrm{y}$, al indicar sus ventajas $\mathrm{y}$ desventajas, ayuda al proceso de toma de decisiones. También es la base para articular una estrategia y crear sucesivamente un plan de acción.

La tecnología considerada en el análisis FODA se elige sobre la base de los resultados del análisis de la matriz de factibilidad. La matriz define los factores clave utilizados en la evaluación de factibilidad. Cada factor tiene su peso particular y se evalúa según cada 
tecnología. Al final, el total del peso calculado definirá el orden del sistema evaluado y las tecnologías evaluadas.

Esta investigación es seguida por una comparación de diferentes tipos de hidro-turbinas. Todos los principios físicos y técnicos son presentados. Estas opciones técnicas se elaboran y comparan a través de diferentes categorías y se comparan a través de la perspectiva de hardware de código abierto y la factibilidad de bricolaje (hazlo tú mismo).

Partiendo del resultado teórico de esta investigación, el componente de aplicación práctica comprende la segunda parte de la tesis. Esto cubre las experiencias de la comunidad de código abierto, formada por la organización sin fines de lucro "Global Anchor". Yo he establecido Global Anchor como entidad legal en el inicio de la investigación técnica. Las siguientes líneas son de la página web de Global Anchor y exponen la visión y misión de la ONG:

"Global Anchor es una organización sin fines de lucro que apoya el desarrollo de soluciones de energía hidroeléctrica de código abierto para la electrificación rural. Queremos ofrecer máquinas robustas, de bajo mantenimiento, de bajo costo, altamente capaces y seguras que aumenten la proliferación de la hidroelectricidad y contribuyan de manera significativa a la electrificación de áreas remotas de manera sostenible. Como parte del movimiento Open Source, esperamos simplificar la creación y el intercambio de máquinas de micro energía hidroeléctrica para satisfacer las necesidades de energía y llevar la luz a áreas remotas."

"Nuestra Visión es capacitar a la gente en áreas rurales / remotas / fuera de la red para satisfacer sus necesidades energéticas de una manera respetuosa con el medio ambiente, renovable y autosostenible. Nuestra Misión es desarrollar soluciones hidroeléctricas pico, hacer accesible nuestro conocimiento, animar a las personas a 
usar este conocimiento para construir sus propias turbinas (Do It Yourself Construction Kits) y así apoyar la electrificación rural (Global Anchor, 2013).”

Con Global Anchor, esta investigación está diseñando un espacio para el desarrollo de una turbina hidroeléctrica pico y sus componentes necesarios. Si desea diseñar y construir su propio sitio hidroeléctrico, entenderlo desde cero y si usted mismo desea operar dicho sistema, encontrará la información abierta y de libre acceso en la página web globalanchor.org. Los manuales de Global Anchor incluirán todo el proceso de implementación del proyecto, desde los pasos iniciales (mediciones de campo y evaluación del sitio) hasta directrices de diseño y selección para cada componente del sistema (obras civiles, conductos forzados, generador, sistema de gobernadores, distribución eléctrica). El enfoque de los manuales de Global Anchor será la documentación del proceso de fabricación de la turbina, que incluye los planos de fabricación y archivos CAD, así como la documentación de apoyo de imagen y video. Esta documentación es considerada como uno de los resultados prácticos de esta investigación.

El estudio se divide en ocho capítulos principales. El primer capítulo es una introducción personal y temática a la investigación. El segundo capítulo se refiere a la crisis global a la que nos enfrentamos y también puede ser visto como parte de mi motivación para el trabajo. En el tercer capítulo, el paradigma moderno se ilustra en relación a cómo la crisis global podría estar interconectada con los valores de una economía moderna. El capítulo cuatro describe el concepto, la idea y los orígenes del hardware de código abierto y de código abierto desde diferentes perspectivas. Particularmente, las ideas centrales del hardware de código abierto y su enfoque post-capitalista se analizan en este capítulo. Este capítulo también presenta ejemplos de proyectos de OSH, compara los dos enfoques y describe las tendencias de la sociedad hacia la apertura y la colaboración. El capítulo cuatro también describe las 
características de los proyectos de código abierto y los compara con los sistemas vivos de la naturaleza. Además, se desarrolla el enlace con la electrificación rural. El capítulo cinco se centra en la situación actual de la electrificación remota a nivel mundial. Esta sección también ofrece una perspectiva de los enormes déficits, así como el potencial, de la electrificación rural, y trata brevemente con posibles soluciones de energía renovable para la electrificación fuera de la red. La perspectiva de este capítulo será más técnica y comparará los conceptos físicos de transformar la energía desde una perspectiva de factibilidad a un enfoque aplicable al "hágalo usted mismo".

El capítulo seis destaca las diferencias técnicas de diferentes tipos de turbinas y los compara bajo la perspectiva del hardware de código abierto. El capítulo seis detalla el estudio de factibilidad de las soluciones de electrificación remota de código abierto. Considera los aspectos técnicos, económicos, legales, organizativos y de programación, y proporciona una estrategia o una hoja de ruta de cómo iniciar tal esfuerzo. Esta sección ofrece el análisis fundamental y los resultados para su aplicación práctica. En el capítulo siete se diseña y se presenta una turbina hidroeléctrica. Esta parte acompaña también el trabajo práctico del diseño hidráulico, ingeniería mecánica, diseño de productos, fabricación de prototipos y la instalación de la ONG Global Anchor, una plataforma para la energía hidroeléctrica de código abierto para la electrificación de áreas remotas. La tesis cierra con una síntesis del proyecto de investigación, y ofrece una perspectiva hacia el futuro con recomendaciones para una mayor aplicación práctica y próximos pasos.

Ni la metodología, ni el tema en sí, es único o nuevo. La energía hidroeléctrica se ha utilizado ahora por varios cientos de años. Del mismo modo, la idea y el enfoque de código abierto es un desarrollo joven en la historia de la humanidad, pero también no es nuevo. Ya hay muchas 
investigaciones sobre código abierto. La singularidad de esta investigación es la combinación de los dos temas: El enfoque de código abierto y la electrificación de área remota. La perspectiva multidisciplinaria hace que la investigación sea significativa y prácticamente aplicable, a través de un producto con licencia de código abierto para la electrificación rural.

\subsection{Aportaciones Originales}

En el marco de esta tesis se elaboró una evaluación de la crisis global. En este capítulo es muy visible que nuestro modo de vida actual basado en el crecimiento y el consumo exige cada vez más recursos. Recursos, que están limitados en un sistema limitado que se llama planeta. Como resultado de la forma en que vivimos estamos luchando con el desaparecer de las especies y la naturaleza, la contaminación del aire y el océano, el calentamiento global y una pérdida crucial de sentido.

Con la investigación del enfoque de código abierto la tesis pretende destacar las similitudes con los procesos naturales y con los "nuevos" campos científicos. Además, la disertación elabora el consumo y la distribución de energía con un enfoque en la energía eléctrica. Un resultado de esto es el hecho de que la energía eléctrica está distribuida de una manera desigual. El consumo de energía de los países del Norte Global es múltiple en comparación con los países ubicados en el Sur Global. También es muy visible que hay grandes diferencias entre la población en zonas rurales y urbanas. Las personas con el menor acceso a la energía eléctrica se encuentran en las zonas rurales del África subsahariana.

A partir de estas precondiciones, la tesis investiga posibles soluciones de energía renovable para la electrificación rural. El viento, la energía solar, la hidroeléctrica y el biogás se comparan en diferentes categorías con una matriz de factibilidad. Estas diferentes categorías 
se ponderan con factores de ponderación. El resultado de este punto de referencia es que la energía hidroeléctrica es la tecnología más apropiada para usar en un enfoque de código abierto. El análisis FODA confirmó este resultado. La investigación adicional en hidroeléctrica para la electrificación rural describe los diferentes tipos de turbinas utilizadas en energía hidroeléctrica y compara entre otras categorías sus campos de aplicación, principios de trabajo, complejidad y costos. Este profundo análisis de diferentes turbinas de energía hidroeléctrica se vuelve a hacer desde la perspectiva del enfoque de hardware de código abierto y la aplicabilidad de hardware de código abierto. La turbina "cross flow" resulta como el tipo de turbina más factible y cierra la parte teórica de la disertación.

La parte práctica de la disertación cubre el diseño, la fabricación y las pruebas de una turbina de este tipo. Esto se acompaña de la fundación de la entidad legal Global Anchor, que es una organización sin fines de lucro, dedicada al hardware de código abierto. Aparte de los requisitos legales, fue también importante hacerse visible con la idea, con la turbina y con la ONG. Por lo tanto, se encontró un nombre, se diseñó un logotipo, se elaboró un concepto de marketing y se implementó un sitio web. Toda la información relacionada con la energía hidroeléctrica de código abierta está disponible en picocrossflow.com. Esto es también el caso de la turbina hidráulica de código abierto en sí, que se llamó Pico Cross Flow.

La investigación de diferentes diseños de turbinas de flujo cruzado, los fondos físicos, teóricos y los supuestos de caso de uso del rango de aplicación resultaron en un diseño preliminar. Después de varias revisiones de diseño se necesitó un taller para fabricar un primer prototipo. Esto fue terminado en Managua Nicaragua a finales de 2014. El Pico Cross Flow es una turbina de flujo cruzado con un rango de aplicación de 10 - 30 litros por segundo de flujo y una diferencia de altura entre 8 y 30 metros. Según las condiciones reales del sitio 
de la altura y el flujo de la potencia de alrededor de 2 kilovatios podría ser extraído de la turbina. Con la información teórica y un prototipo fabricado era importante hacer pruebas a largo plazo, que desafortunadamente hasta ahora no fue posible de encontrar. Debido a eso la decisión fue probar la turbina en un laboratorio de mecánica. En verano de 2016 la Universidad Técnica de Viena confirmó el rango de aplicación y la potencia de salida de la turbina mientras la probaron en su laboratorio en el marco de una tesis de licenciatura.

El conjunto completo de información sobre el diseño de la turbina, el estado del proyecto, la información de fondo y los próximos pasos para llevar adelante el proyecto se pueden encontrar en el sitio web www.picocrossflow.com.

\subsection{Desafios y Futuras Líneas de Investigación}

Con la aplicación práctica de esta investigación muchas cosas han sido logradas en diciembre de 2016. Al mismo tiempo, todavía queda mucho por hacer. Los próximos pasos del proyecto se pueden dividir en cuatro ramas principales de acción:

\section{A) Publicación del estado real del proyecto}

Con los resultados de las pruebas y una solución técnica clara es importante capacitar a la gente para reconstruir la turbina. El requisito necesario es la publicación de los expedientes. Por lo tanto, una acción concreta será crear un modelo 3D, dibujos de fabricación y una documentación de fabricación. Con estos archivos publicados y disponibles en línea, las personas son capaces de fabricar una copia de la turbina y, finalmente, contribuir con su experiencia en el proyecto. 


\section{B) Actualizaciones de diseño}

Otra rama de la acción concreta es la optimización del diseño hidráulico y mecánico. En el marco de otra tesis de licenciatura en la Universidad de Viena se podría abordar la optimización hidráulica de la turbina. La actualización en el diseño hidráulico y un aumento de la tasa de eficiencia seguirá una adaptación del diseño mecánico. Este proceso depende de la disponibilidad del laboratorio hidráulico de la Universidad de Viena, que está a punto de instalarse en un nuevo edificio. Esto podría chocar con la acción D), implementar la turbina en un sitio piloto.

Otra opción surgió a través de un contacto con la Universidad de Stuttgart, donde parece posible que los estudiantes hagan la optimización hidráulica a través de la simulación. Estas dos opciones tienen que ser evaluadas.

\section{C) Financiamiento y Finanzas}

El financiamiento es importante y necesario para aumentar la mano de obra, crear y obtener archivos de documentación y planos de fabricación en línea. Mediante el financiamiento, la gente podría dedicar su trabajo a Pico Cross Flow, que acelera la producción general de proyectos, crea más visibilidad y atrae más gente. En segundo lugar para las pruebas de largo plazo es importante tener un "sitio piloto". El financiamiento inicial y la suficiente flexibilidad financiera son factores cruciales para la subsistencia y el éxito de la idea de la energía hidroeléctrica de código abierto.

\section{D) Pruebas a largo plazo}

Al tener la turbina probada con éxito en un entorno de laboratorio artificial, es importante experimentar el rendimiento de las turbinas en "condiciones reales". Las pruebas a largo plazo en "condiciones de trabajo reales" ofrecen otra perspectiva sobre la estabilidad y el 
rendimiento. Esto se puede hacer en un "sitio piloto" donde la turbina puede ser probada en un período de varios meses en casos de uso real. Para eso la disponibilidad de las partes eléctricas del sistema de energía hidroeléctrica es necesaria. Un generador y un sistema de gobierno son inevitables. Referiendo al lado eléctrico del esquema de energía hidroeléctrica, se han hecho consideraciones preliminares y varias opciones surgieron. Ninguna de estas opciones es posible sin apoyo financiero.

En conclusión, para realizar pruebas a largo plazo es necesario obtener apoyo financiero y encontrar un sitio conveniente para hacerlo.

\section{Principales desafíos y perspectivas}

Debido al hecho de que la energía hidroeléctrica es una tecnología compleja dependiendo de los recursos naturales, parece muy difícil conseguir que la gente contribuya a la comunidad de Pico Cross Flow. La energía hidroeléctrica es un nicho muy pequeño. Las personas involucradas en la energía hidroeléctrica se enfrentan a la ingeniería eléctrica y mecánica. Además, las personas que tienen recursos naturales adecuados no son siempre las mismas personas que son técnicos capacitados e interesados en hardware de código abierto. Debido a la compleja tecnología, la dependencia de las condiciones específicas del sitio natural y el beneficiario, principalmente viviendo en el Sur Global, parece muy difícil conseguir que la gente se sienta atraída por la idea del proyecto. Como consecuencia, una contribución más específica y orientada a la tarea es muy deseable.

Otro desafío en términos de dinero se hizo visible. Desafortunadamente, el beneficio financiero de una turbina de código abierto para proyectos de energía hidroeléctrica pico es muy limitado. El beneficio financiero es sólo un pequeño porcentaje de los costos totales del proyecto de energía hidroeléctrica. Como consecuencia, un objetivo podría ser proporcionar 
el equipo electromecánico completo como un kit "hágalo usted mismo" en un formulario de licencia de código abierto. Haciendolo así los costes serían más bajos pero el problema permanece. Los costos del proyecto sólo se podrían reducir a un porcentaje específico (dependiendo de las condiciones naturales del sitio) de los costos totales del proyecto, resultando que el beneficio financiero es sólo un pequeño porcentaje de los costos generales del proyecto.

La energía hidroeléctrica de código abierto, vista desde una perspectiva económica, no es muy beneficiosa. El beneficio radica en la tecnología descentralizada y respetuosa con el medio ambiente y el empoderamiento de las personas, que está relacionado con la transferencia de conocimientos.

Una turbina hidráulica de código abierto podría ser construido fácilmente en un taller o en casi todos los espacios de hackers en el Norte Global. El reto es que no es "necesario" en el Norte Global. En Nueva York, París o Tokio la gente construye cortadores láser, drones o robots. Tal vez se necesita más tiempo en la electrificación rural que en otras áreas donde el hardware de código abierto se desarrolla de forma más dinámica. Con estas máquinas mencionadas la gente se conecta más fácilmente y las utiliza en su tiempo libre y en forma de un hobby. Este no es el caso de la energía hidroeléctrica. Muy poca gente hace la energía hidroeléctrica como su pasatiempo. Esto ya está enraizado en la disponibilidad de sitios naturales y la legislación de uso de la tierra. Es muy difícil encontrar personas que son capaces de implementar una turbina hidráulica en su arroyo detrás de la casa y al mismo tiempo tienen el interés en la tecnología, así como la capacidad financiera para hacerlo. El caso es totalmente diferente para drones o cortadores láser, por ejemplo. Estas máquinas no dependen de condiciones específicas del sitio natural o de las regulaciones legales del uso del agua. 
Sin embargo, creo que la turbina Pico Cross Flow será aplicada e implementada por una gama más amplia de personas cuando haya una aceptación más amplia del hardware de código abierto en la sociedad.

\subsection{Conclusión}

Es muy importante, si no crucial para las próximas generaciones, cambiar nuestra forma de vida. En la evaluación de la crisis mundial es muy visible que el aumento de los residuos, la disminución de especies y el cambio climático cambian las condiciones de vida en este planeta. El estilo de vida basado en el crecimiento y el consumo no conduce a la felicidad e implica un servidor y daños irreversibles en el medio ambiente. Las ideas de código abierto podrían apoyar una transformación del estilo de vida basado en el crecimiento y la competencia hacia una sociedad más colaborativa.

Además, la disertación desarrolla el consumo y la distribución de energía, resultando en un grave desequilibrio de acceso a la energía eléctrica. Especialmente en las zonas rurales del Sur Global, el acceso a la electricidad es muy limitado. La energía es una necesidad básica y cambia la vida de las personas esencialmente. Por eso, la parte práctica de la tesis trata de soluciones técnicas para la electrificación rural y elabora su factibilidad desde la perspectiva del hardware de código abierto.

El desarrollo de una matriz de comparación de tecnología ayuda a elaborar y destacar que el suministro de energía renovable con energía hidroeléctrica parece ser el más apropiado para 
un enfoque de código abierto. En un segundo paso, el análisis FODA investiga el tipo de turbina más prometedor, dando como resultado un diseño de turbina de flujo cruzado.

La aplicación práctica de una hidroeléctrica de código abierto para la electrificación rural es considerada técnicamente posible. Al mismo tiempo, parece que este producto específico es muy difícil de implementar y obtener impulso a la misma. Hay múltiples causas para esto. Por un lado, es un producto en un nicho muy especial. Por otro lado, está tocando una tecnología muy compleja y, al mismo tiempo, hasta ahora sólo ayuda a ahorrar dinero en una fracción de los costos generales del proyecto. Esto significa que la idea en general es factible y también aplicable. Pero al mismo tiempo podría ser que no es el momento para este cambio, ya que el hardware de código abierto no está tan desarrollado como el software de código abierto es hoy en día. Parece que el hardware de código abierto necesita un par de años más para llegar a la sociedad en un rango más amplio. Cuando este es el caso, la probabilidad crece que las diferentes ONGs que están trabajando en el área de desarrollo sostenible apliquen y usen la tecnología de código abierto en lugar de diseños de turbinas patentados.

Parece que el hardware de código abierto se está propagando lentamente pero constantemente en varios campos de nicho y mercados potenciales, donde la diferencia financiera entre las soluciones tradicionales y las de código abierto es enorme. Sin embargo, el proceso es muy lento, especialmente en campos como la electrificación rural, donde el mercado es muy limitado y el beneficio financiero de las soluciones de código abierto es marginal.

Queda probada la hipótesis de esta investigación doctoral, tal que podemos afirmar que, el hardware de código abierto, puede ser un instrumento de crecimiento para las sociedades, que, superando modelos capitalistas, fomente la colaboración, la apertura y el desarrollo de 
las personas y las comunidades desde una perspectiva de solidaridad pero también de sostenibilidad y de mutuo crecimiento. En cuanto a un modelo técnico concreto y aplicado, en el ámbito de la generación de energía, renovable, limpia, sostenible y autónoma, ciertos desarrollos a pequeña escala -como es el caso de la turbine Pico Cross Flow que se diseña y desarrolla en esta investigación doctoral- pueden aportar soluciones de gran valor, de gran aplicabilidad y que aporten soluciones a un gran número de personas y comunidades, evidenciando la potencia del ingenio y la gestión de las escalas.

Sin embargo, la idea de código abierto es una contribución importante a un modo de desarrollo económico más sostenible y probablemente tendrá una mayor influencia en muchos campos en el futuro. 


\section{Appendix}

\subsection{Website Pico Cross Flow}

The following text depicts the core idea of the project and is published on the picocrossflow.com website (November 2016):

The Pico Cross Flow is an open source cross flow turbine. We designed it to fill the need of people with no access to electricity, who lack a safe, robust, affordable and highly-capable machine. Unlike others the Pico Cross Flow comes fully loaded with knowledge to build, run and maintain the whole hydroelectric scheme.

As part of the Open Source movement we hope to simplify the creation and sharing of pico hydropower solutions to satisfy energy needs and bring light to remote areas.

We want to provide robust, low-maintenance, low-cost, efficient, highly-capable and safe machines that will increase the proliferation of hydroelectricity and make a significant contribution to remote area electrification in an environmentally sustainable way.

Currently Pico Cross Flow is at a stage of testing a working prototype at and with the help of the Hydrodynamic Laboratory of the Technical University of Vienna. We want to get data to determine its efficiency curves and power output. Once we get a prototype that meets acceptable working conditions, we will proceed to manufacture a market-ready product and publish its design. We want to ultimately have a product that is robust and highly efficient, requiring minimal specialized tools to build and assemble at a very low cost.

\section{Vision Statement}

Our Vision is to empower people and NGOs working in rural, remote, off-grid areas to satisfy energy needs in an environmental friendly, renewable and self-sustainable way. Pico Cross Flow aims to be at the forefront of developing innovative pico hydropower solutions for people at the base of the social economic pyramid.

\section{Mission}

Our Mission is to develop pico hydropower designs, make our knowledge accessible, encourage people to use this knowledge to build and set up pico hydropower schemes by themselfes (Do It Yourself Construction Kits) and thereby support rural, decentralized, environmentally friendly and sustainable energy transformation.

Product

Instead of supporting costly kilometer long grid extension Pico Cross Flow takes the absence of infrastructure as an opportunity to develop decentralized energy supply on a very small scale. Aimed with this logic Pico Cross Flow is developing a pico cross flow turbine with a maximum power output of $5 \mathrm{~kW}$. 
Social Impact

If successful not only would Pico Cross Flow replace diesel generators and have a positive impact on the greenhouse gas emission but it would also have a positive impact on the lives of many people by setting free large amounts of human time and labor and by connecting people with modern communication. We believe that a Pico Cross Flow hydroelectric scheme supports to attain decent quality of life through meaningful innovation.

\subsection{Hydraulic Calculation and Design}

This chapter describes the geometries and calculation on which the hydraulic design of the

Pico Cross Flow is built on.

Predefined values:

Head H

20

$[\mathrm{m}]$

Flow Q

20

$[1 / \mathrm{s}]$

Revolution $\mathrm{n}$

$>1000$

[rpm]

Assumptions:

$\begin{array}{ll}\text { Runner outer diameter D } & 0.12 \\ \text { Absolute velocity angle } \alpha_{1} & 16 \\ \text { Relative velocity angle } \beta_{2} & 30 \\ \text { Nozzle coefficient k } & 0.967 \\ \text { Diameter ratio } v & 0.66 \\ \text { Number of blades z } & 26\end{array}$

$[\mathrm{m}]$

Number of blades z

Absolute speed of water jet $\mathrm{c}$ :

$c=k x \sqrt{2 x g x H}=0.967 x \sqrt{2 \times 9.81 \times 20}=19.16[\mathrm{~m} / \mathrm{s}]$

Turbine rotational speed $\mathrm{u}$ :

$u=\frac{1}{2} x$ c $x \cos \alpha=\frac{1}{2} x 19.16 x \cos 16=9.28[\mathrm{~m} / \mathrm{s}]$

Turbine speed n:

$\omega=2 \times \pi \times n$ and $u=r \times \omega$ which results in either

$n=\frac{\omega}{2 \times \pi}=\frac{60 \times u}{D \times \pi} \quad$ or $\quad D=\frac{60 \times u}{n \times \pi} \quad$ depending on the predefined value. 
In this case the assumption for the outer diameter $\mathrm{D}$ was made, which results in:

$n=\frac{60 \times u}{D \times \pi}=\frac{60 \times 9.28}{0.12 \times \pi}=1477[\mathrm{rpm}]$

Turbine inner diameter $\mathrm{d}$ :

$d=D x v=0.12 \times 0.66=0.079[\mathrm{~m}] \quad \rightarrow$ selected $80[\mathrm{~mm}]$

Inlet width of the runner b:

$b=\frac{Q \times 360^{\circ}}{D \times \pi \times \Phi^{\circ} \times \sqrt{2 \times g \times H} \times \sin \alpha}=\frac{0.02 \times 360^{\circ}}{0.12 \times \pi \times 90^{\circ} \times 19.81 \times \sin 16}=0.043[\mathrm{~m}]$

$\rightarrow$ Runner width selected $60[\mathrm{~mm}]$

Blade geometry and pitch circle radius $r_{p}$ :

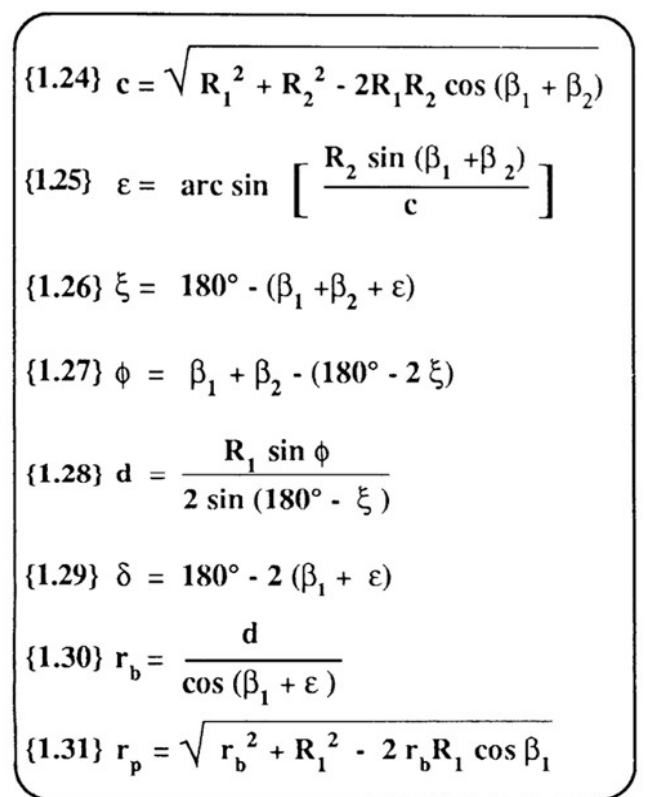

Calculation of blade geometry coefficients according to the equations on the left and the sketch on the following page.

\begin{tabular}{|l|c|c|l|}
\hline & $\mathrm{c}$ & 0.087 & {$[\mathrm{~m}]$} \\
\hline & $\varepsilon$ & 23.421 & {$[$ degree] } \\
\hline & $\xi$ & 36.579 & {$[$ degree] } \\
\hline & $\Phi$ & 13.158 & {$[$ degree] } \\
\hline & $\mathrm{d}$ & 0.0115 & {$[\mathrm{~m}]$} \\
\hline $\begin{array}{l}\text { Segment Angle on } \\
\text { Blade }\end{array}$ & $\delta$ & 73.158 & {$[$ degree] } \\
\hline Curvature Radius & r_b & 0.0192 & {$[\mathrm{~m}]$} \\
\hline Pitch Circle Radius & r_p & 0.0444 & {$[\mathrm{~m}]$} \\
\hline
\end{tabular}




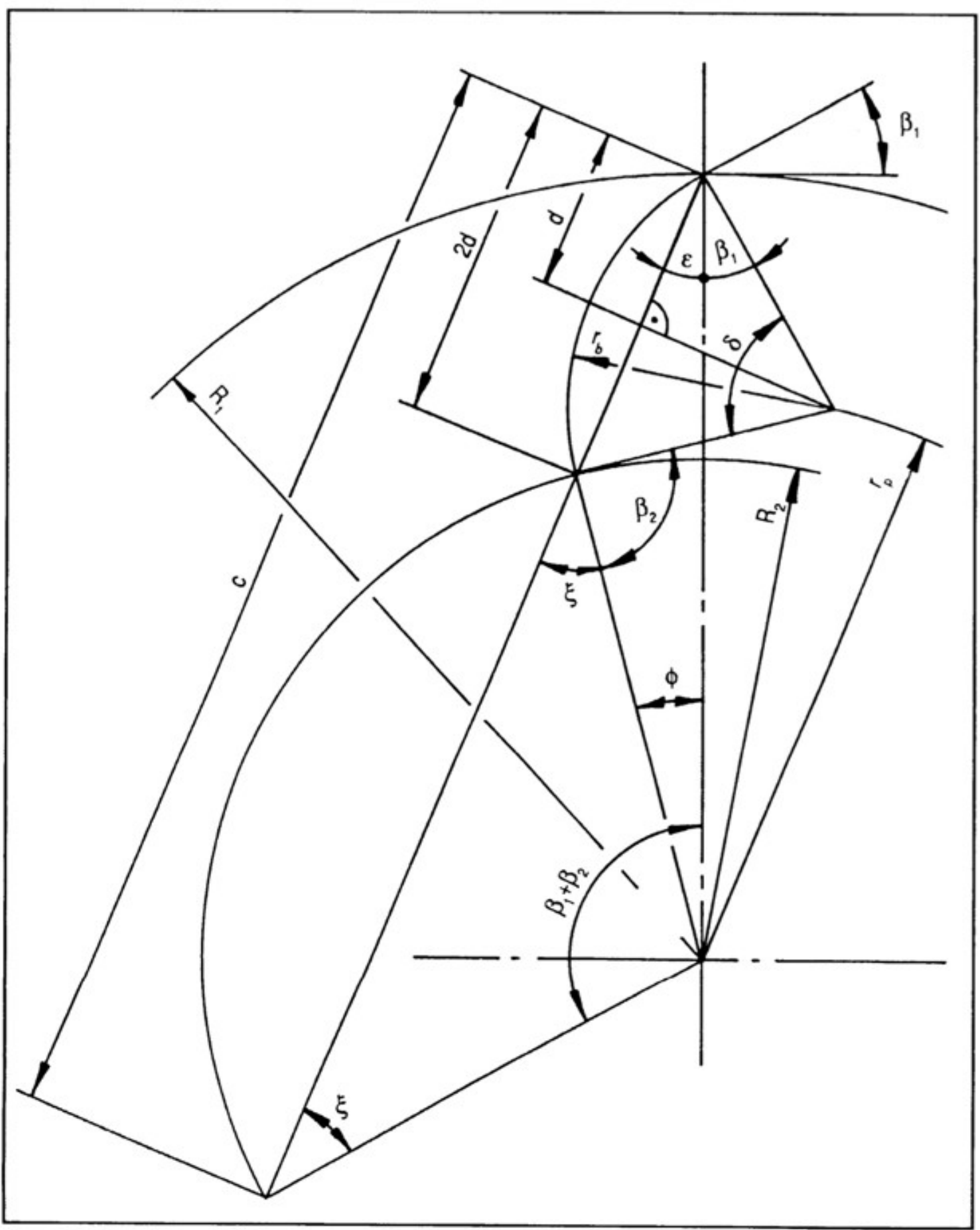

Blade geometry and pitch circle radius (Arter \& Meier, 1990: 24)

Injector geometry:

The injector geometry was calculated according to Arter \& Meier (1990) as visible on the following page. It is important to mention, that the results of $R \Phi$ are highly theoretical 
values. The results for the respective diameter $R \Phi$ was used for the mechanical design of the inlet spiral, but it was adjusted as various angles. It is very difficult to be that precise to have an exact radius of $\mathrm{R} \Phi$ at $68.2[\mathrm{~mm}]$ for example, when the manufacturing facilities do not provide with the appropriate machining tools. Additionally, this is not the intention of the Pico Cross Flow design. Its design is adapted for easy manufacturing and simplified hydraulic design.

\begin{tabular}{|c|c|c|c|c|}
\hline \multicolumn{2}{|c|}{$360^{\circ} \triangleq 2 \pi$} & $\mathbf{r}_{\phi}=\mathrm{e}^{\tan \phi_{0} \phi[\text { rad }]}$ & \multicolumn{2}{|l|}{$\mathrm{r}_{0} \cdot \mathbf{R}_{1}$} \\
\hline deg. & rad. & $r_{0}$ & $R_{\text {o }}$ & \\
\hline 0 & 0 & 1.000 & 60.0 & {$[\mathrm{~mm}]$} \\
\hline 5 & 0.087 & 1.026 & 61.6 & {$[\mathrm{~mm}]$} \\
\hline 10 & 0.175 & 1.053 & 63.2 & {$[\mathrm{~mm}]$} \\
\hline 15 & 0.262 & 1.080 & 64.8 & {$[\mathrm{~mm}]$} \\
\hline 20 & 0.349 & 1.108 & 66.5 & {$[\mathrm{~mm}]$} \\
\hline 25 & 0.436 & 1.137 & 68.2 & {$[\mathrm{~mm}]$} \\
\hline 30 & 0.524 & 1.167 & 70.0 & {$[\mathrm{~mm}]$} \\
\hline 35 & 0.611 & 1.197 & 71.8 & {$[\mathrm{~mm}]$} \\
\hline 40 & 0.698 & 1.229 & 73.7 & {$[\mathrm{~mm}]$} \\
\hline 45 & 0.785 & 1.260 & 75.6 & {$[\mathrm{~mm}]$} \\
\hline 50 & 0.873 & 1.294 & 77.6 & {$[\mathrm{~mm}]$} \\
\hline 55 & 0.96 & 1.327 & 79.6 & {$[\mathrm{~mm}]$} \\
\hline 60 & 1.047 & 1.362 & 81.7 & {$[\mathrm{~mm}]$} \\
\hline 65 & 1.134 & 1.397 & 83.8 & {$[\mathrm{~mm}]$} \\
\hline 70 & 1.222 & 1.434 & 86.0 & {$[\mathrm{~mm}]$} \\
\hline 75 & 1.309 & 1.471 & 88.3 & {$[\mathrm{~mm}]$} \\
\hline 80 & 1.396 & 1.509 & 90.6 & {$[\mathrm{~mm}]$} \\
\hline 85 & 1.484 & 1.549 & 92.9 & {$[\mathrm{~mm}]$} \\
\hline 90 & 1.571 & 1.589 & 95.4 & {$[\mathrm{~mm}]$} \\
\hline 95 & 1.6583 & 1.631 & 97.8 & {$[\mathrm{~mm}]$} \\
\hline 100 & 1.7456 & 1.673 & 100.4 & {$[\mathrm{~mm}]$} \\
\hline 105 & 1.8329 & 1.717 & 103.0 & {$[\mathrm{~mm}]$} \\
\hline
\end{tabular}




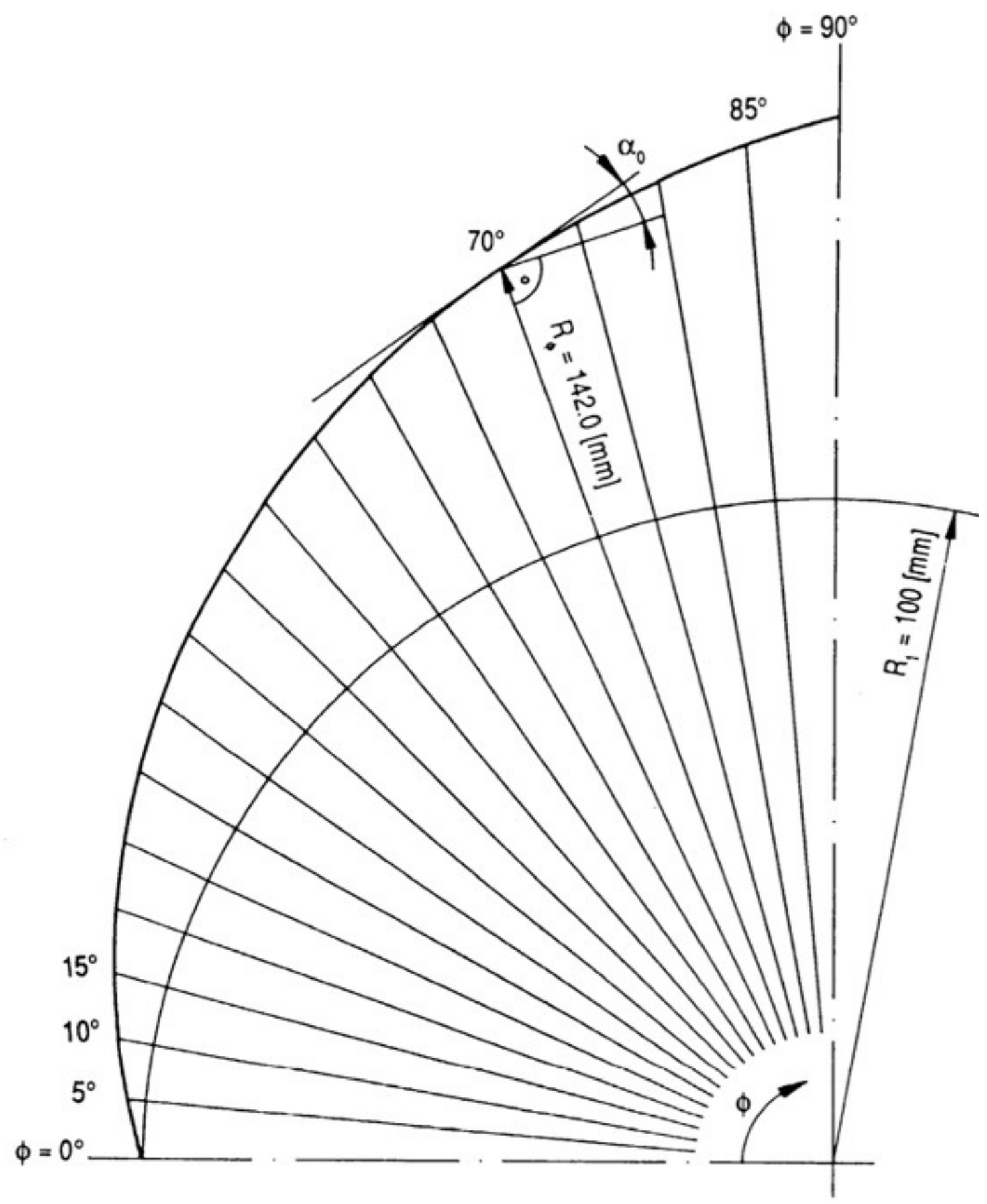

Design of the logarithmic Inlet Spiral (Arter \& Meier, 1990: 26)

All additional turbine specification and calculation results are available on the Pico Cross Flow website: www.picocrossflow.com 


\subsection{Pico Cross Flow Manufacturing Photos}
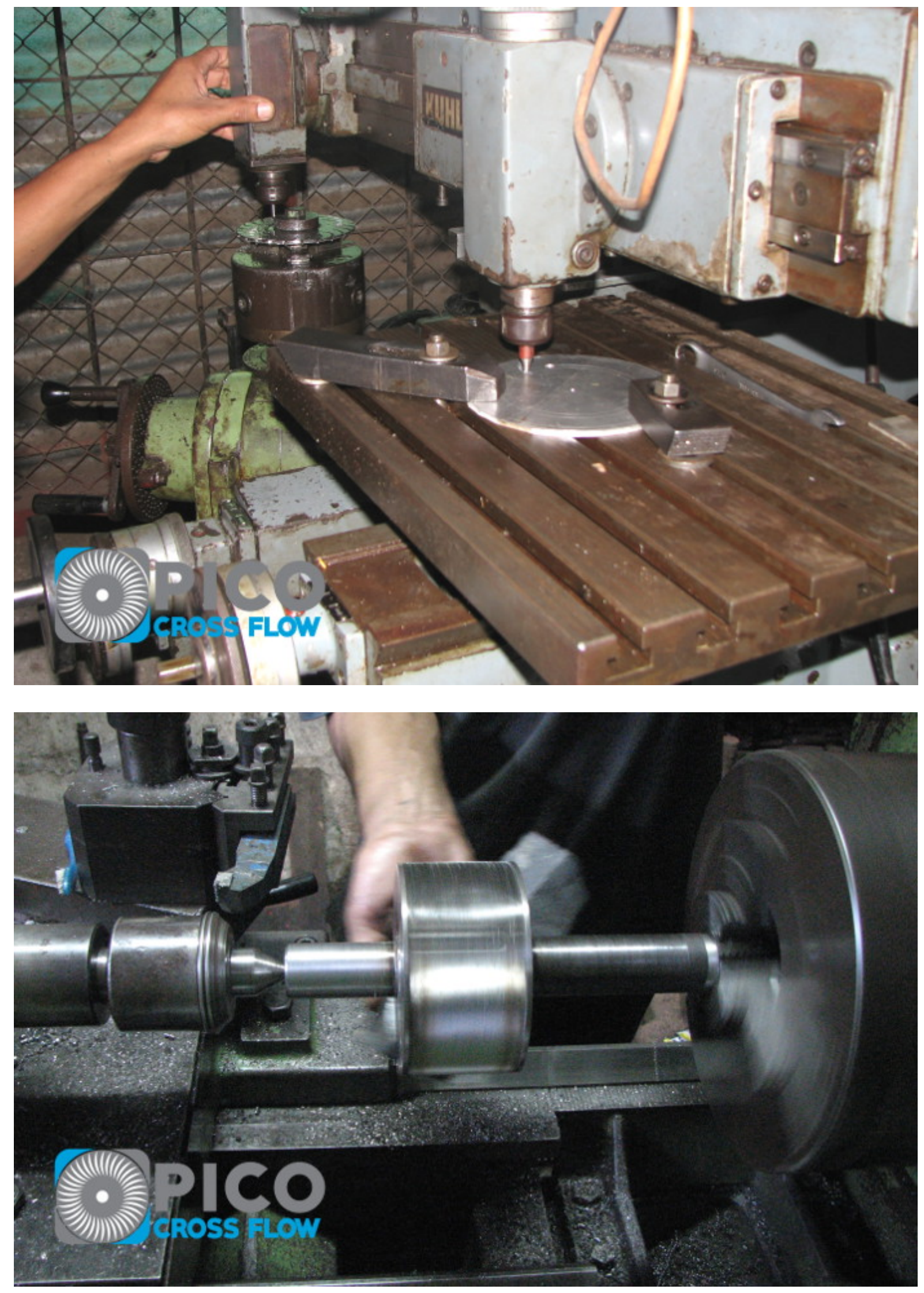
Appendix
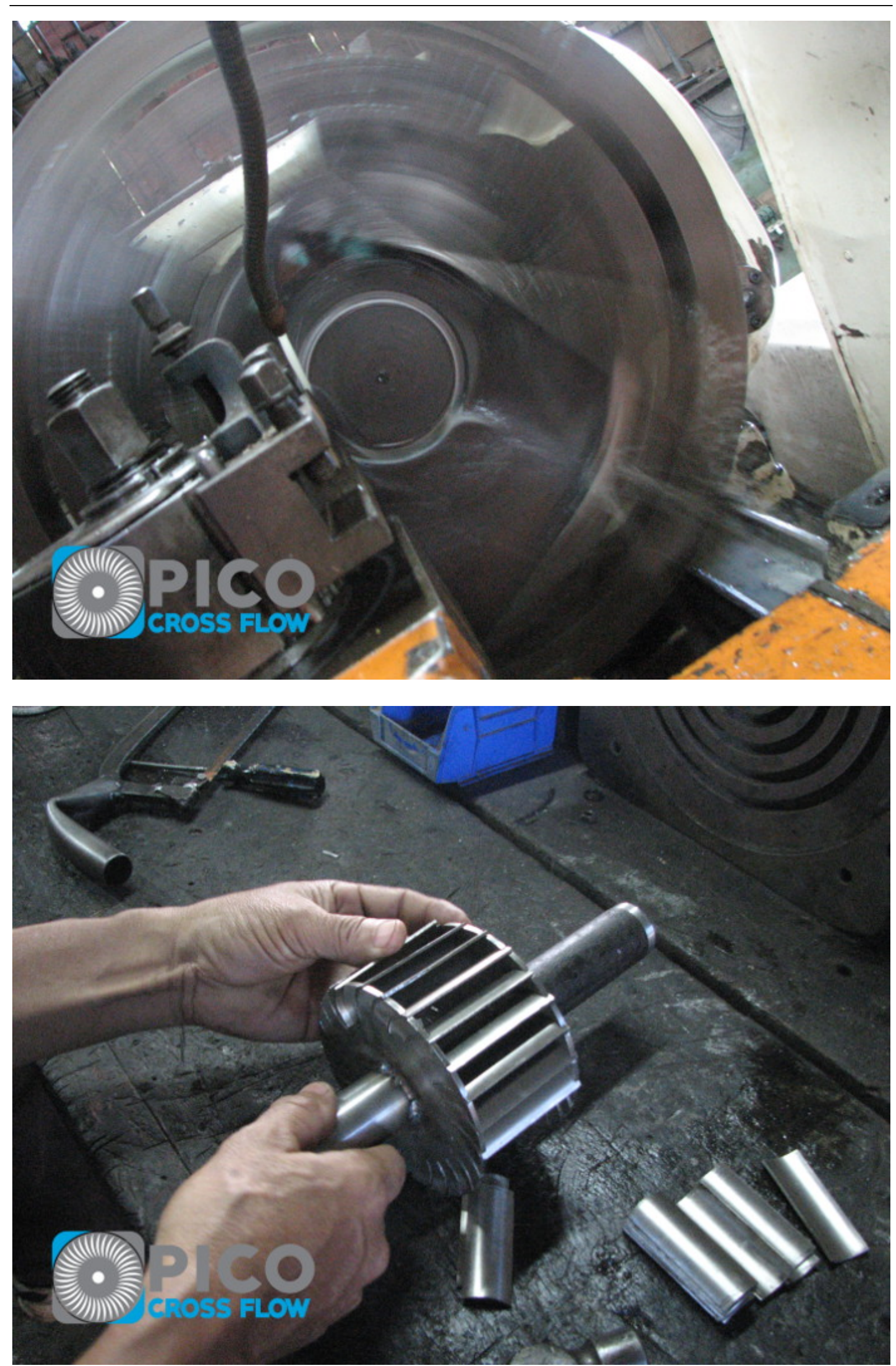


\subsection{Pico Cross Flow 3D Model and Drawings}
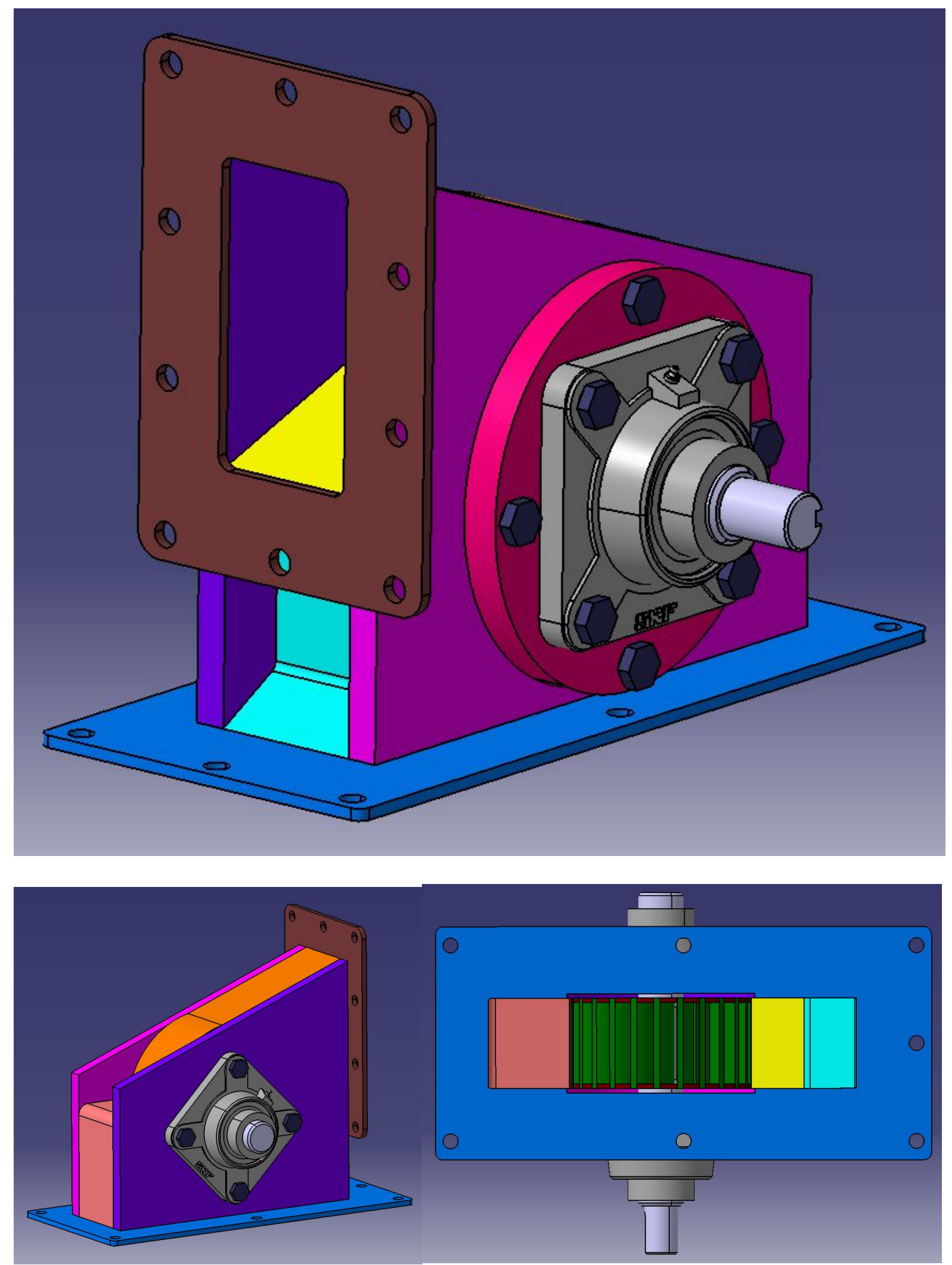


\section{Appendix}

Additional Information:

Scale of all views: $1: 2$

Bearings: $2 x$ flanged $Y$-bearings, cast housing, type: FY 30 TB Number of runner-blades: 24

\section{Expected Operation Range:}

Head: 8 - $30[\mathrm{~m}]$

- Flow: $10-30[1 / \mathrm{s}]$

Power Output: $1-3,5[\mathrm{~kW}]$

* at $H=30[\mathrm{~m}]$ and $F=25[1 / \mathrm{s}]$
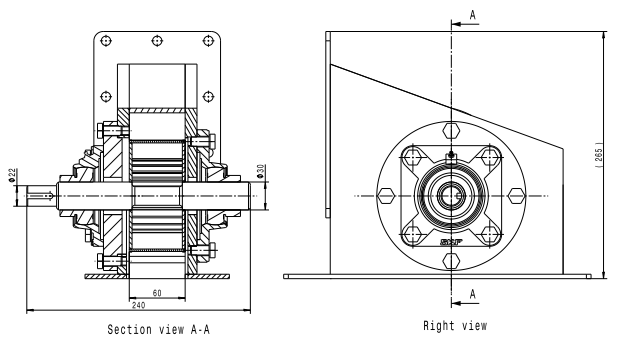

Right vien

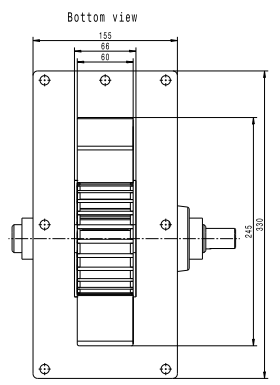

Pico Cross Flow Turbine
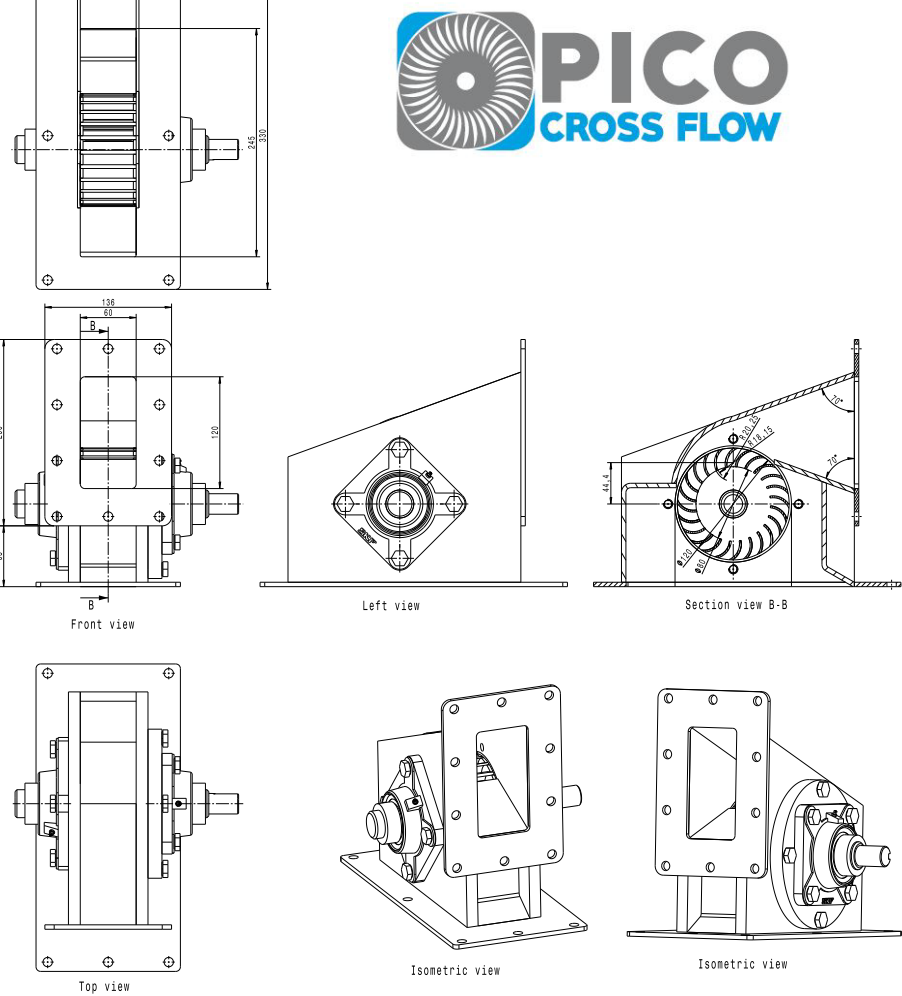

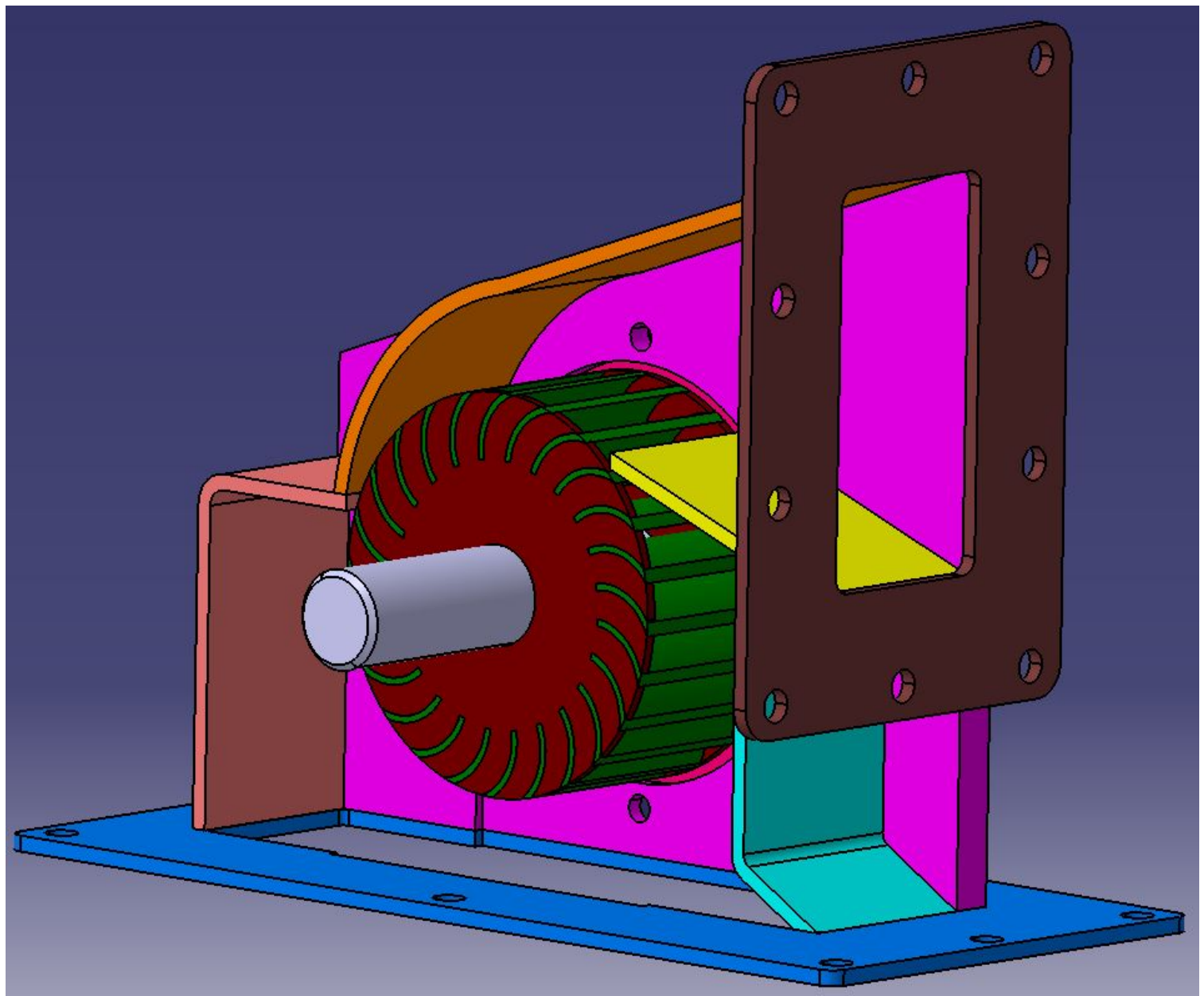




\subsection{Pico Cross Flow Prototype Turbine Photos}
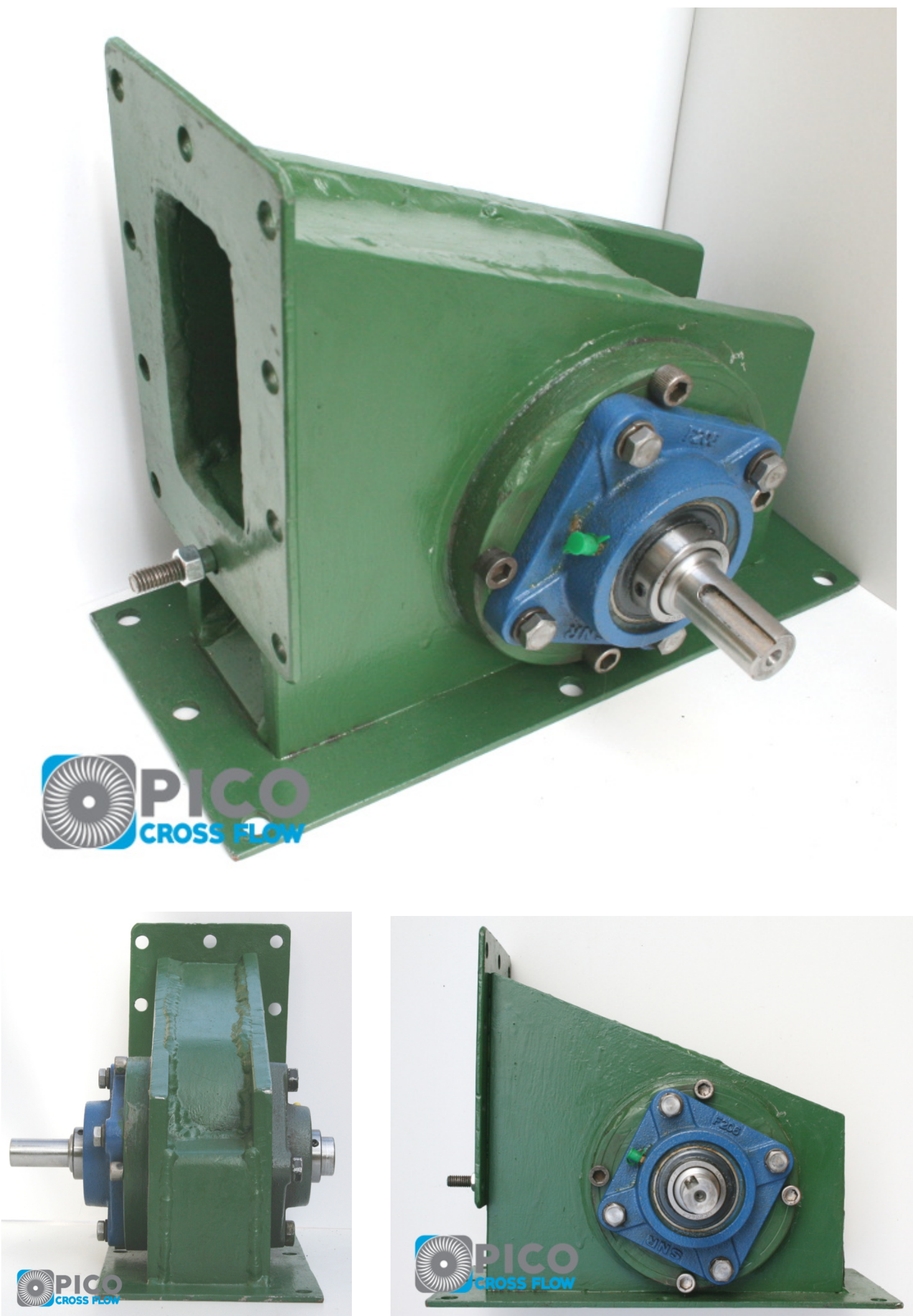
12.6 Hydraulic Testing at the Technical University Vienna Photos
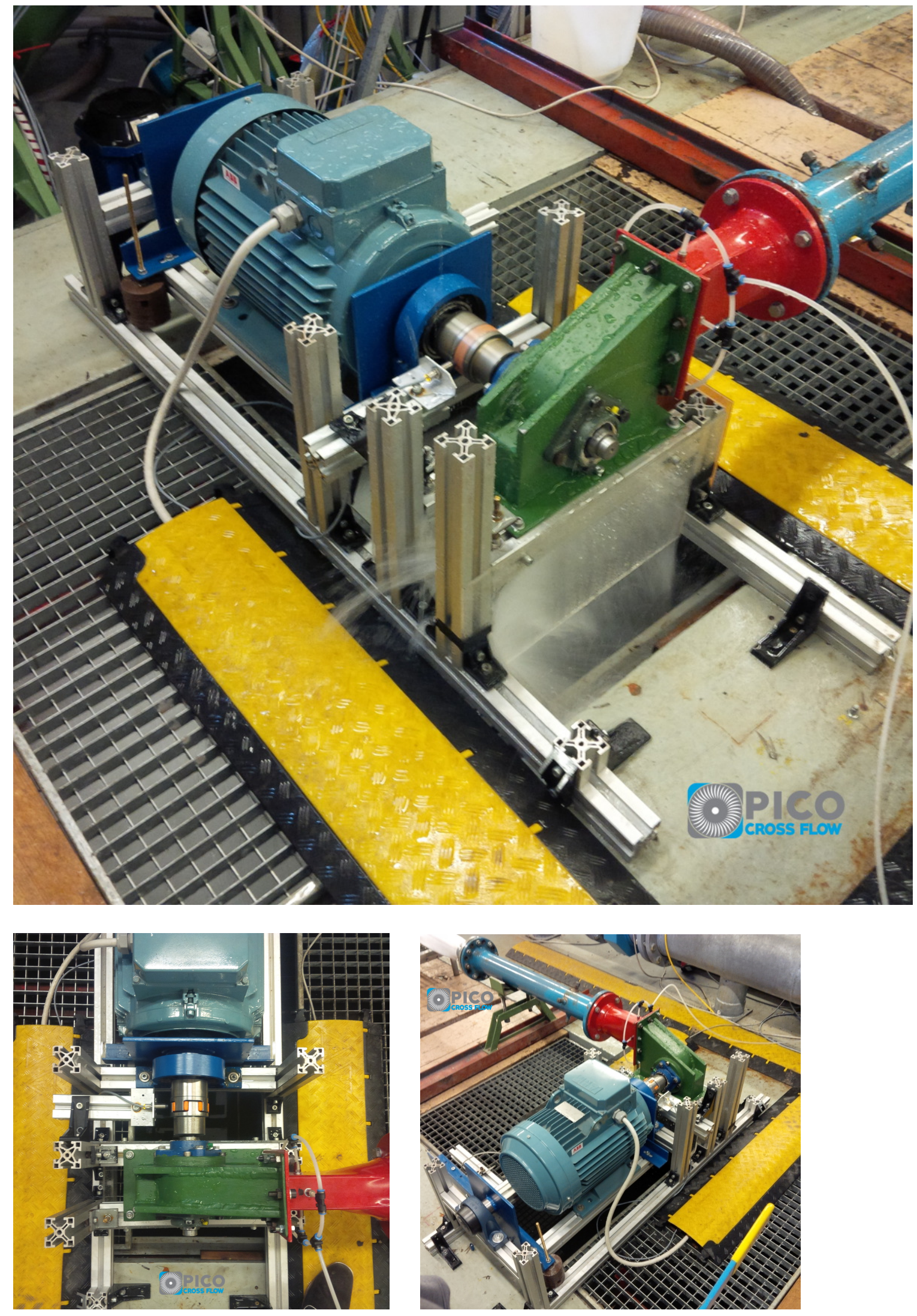


\subsection{Pico Cross Flow Testing Results}

The attached document describes the expected performance of the Pico Cross Flow turbine. This document is also available on the project website: www.picocrossflow.com. All additional turbine specification and ongoing testing results are available on the website as well. 

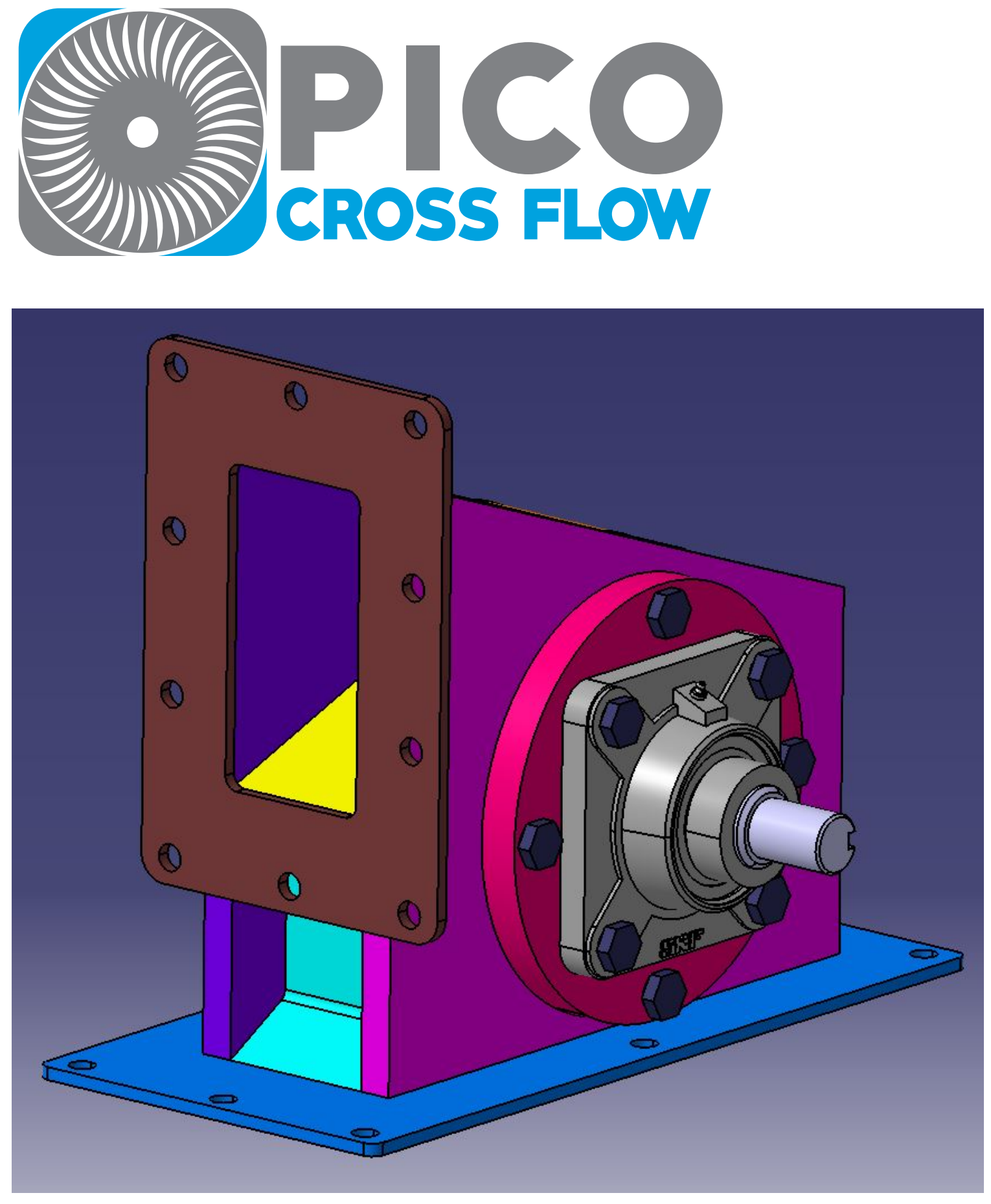

\section{Pico Cross Flow Turbine 3kW Prototype} Application Range

Date: 15.01.2017

Author: Ino Fleischmann 
The following information are the result of the Pico Cross Flow turbine testing at the Technical University of Vienna in June 2016:

\begin{tabular}{|c|c|c|c|c|}
\hline \multicolumn{5}{|c|}{ Ideal speed for different heights } \\
\hline $\mathbf{H}(\mathbf{m})$ & $\mathbf{n}_{\mathbf{~ o p t}} \mathbf{( U / \mathbf { m i n } )}$ & $\mathbf{P}(\mathbf{k W})$ & $\mathbf{Q}(\mathbf{l} / \mathbf{s})$ & $\mathbf{\eta} \%$ \\
\hline 5 & 755 & 0,429 & 17,75 & 49,28 \\
\hline 8 & 958 & 0,889 & 22,46 & 50,48 \\
\hline 10 & 1058 & 1,159 & 24,61 & 48,06 \\
\hline 12 & 1211 & 1,570 & 27,18 & 49,64 \\
\hline 15 & 1364 & 2,196 & 30,48 & 48,95 \\
\hline 20 & 1618 & 3,492 & 34,94 & 50,91 \\
\hline 25 & 1824 & 4,951 & 38,95 & 51,95 \\
\hline 30 & 1981 & 6,490 & 42,89 & 51,43 \\
\hline
\end{tabular}

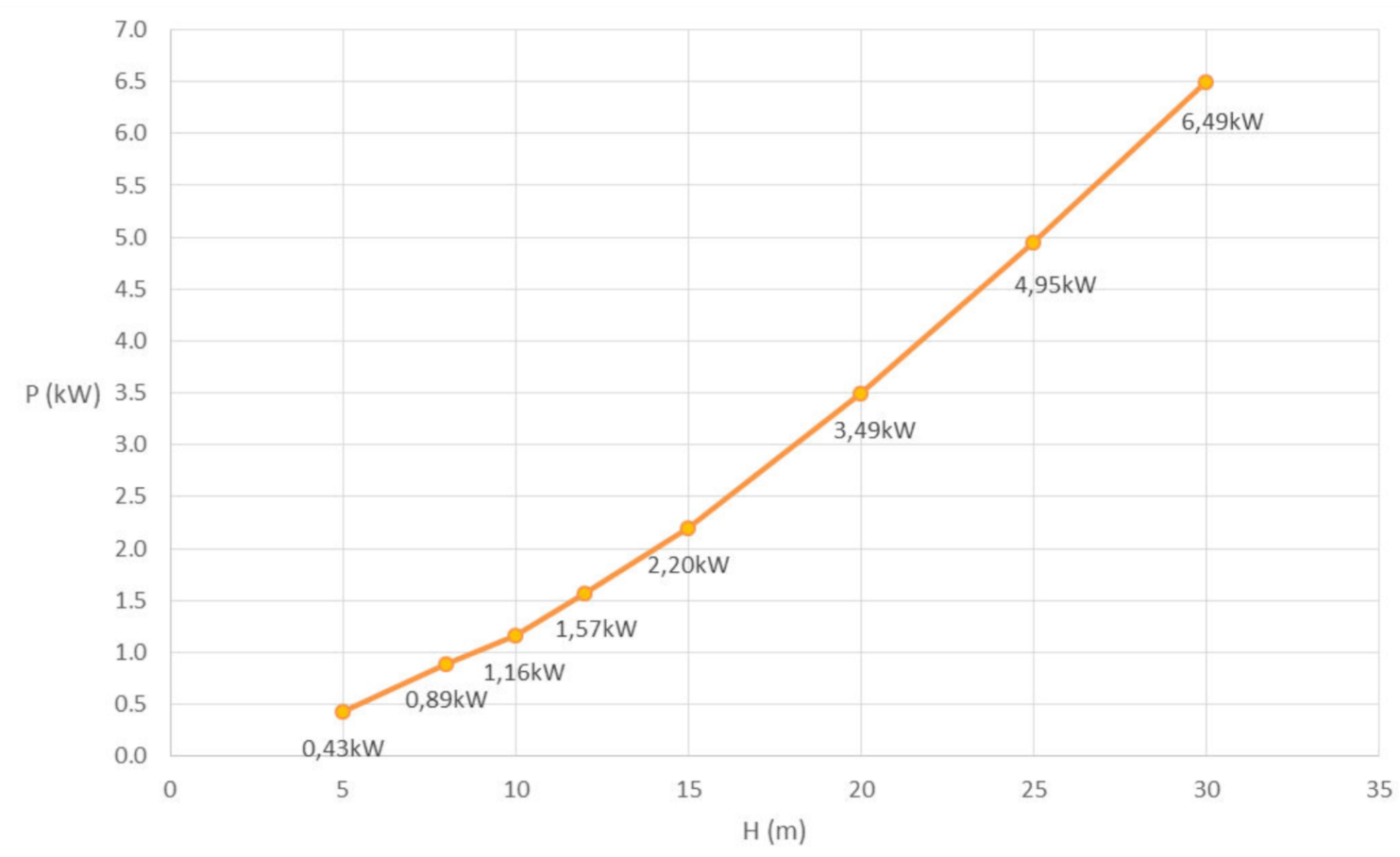



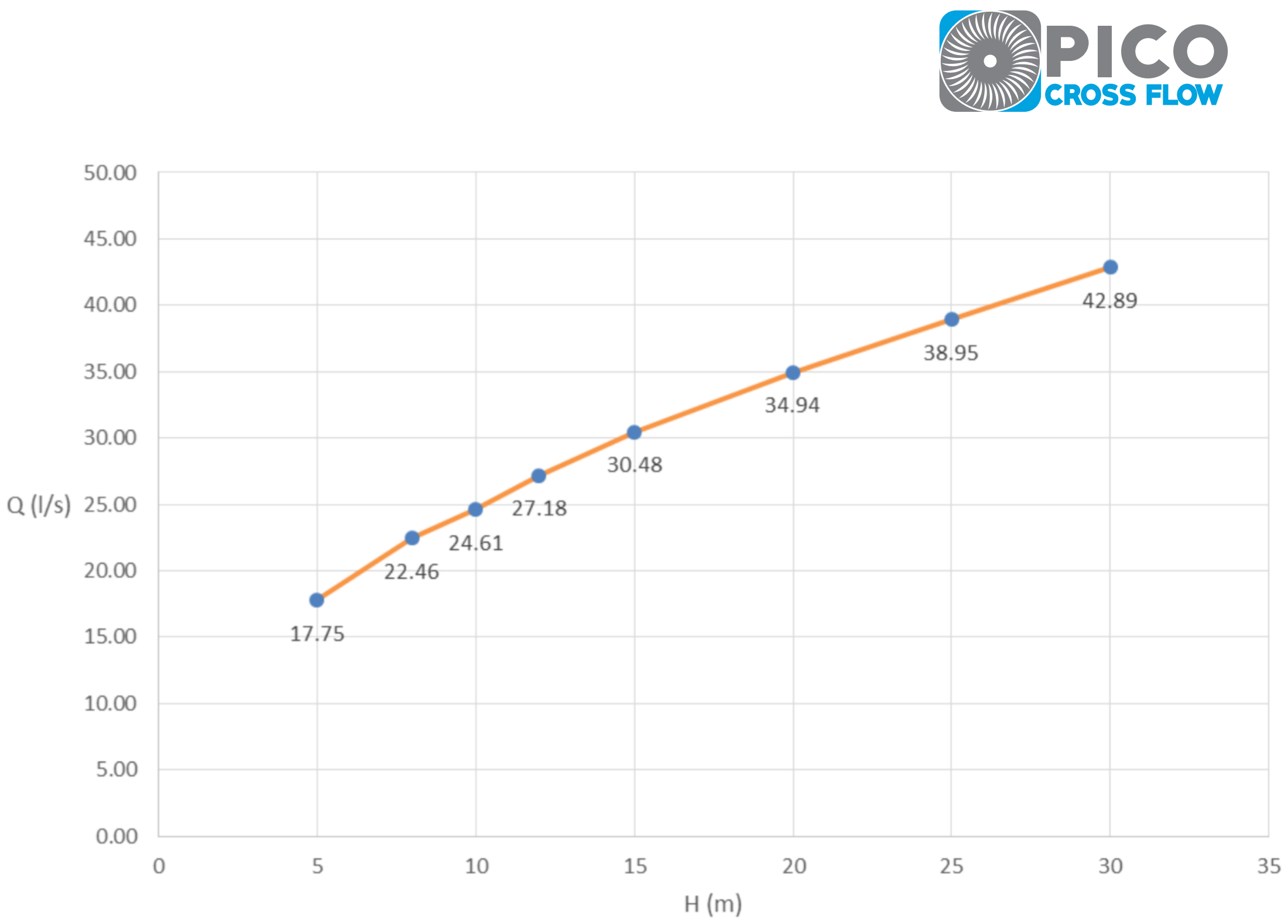

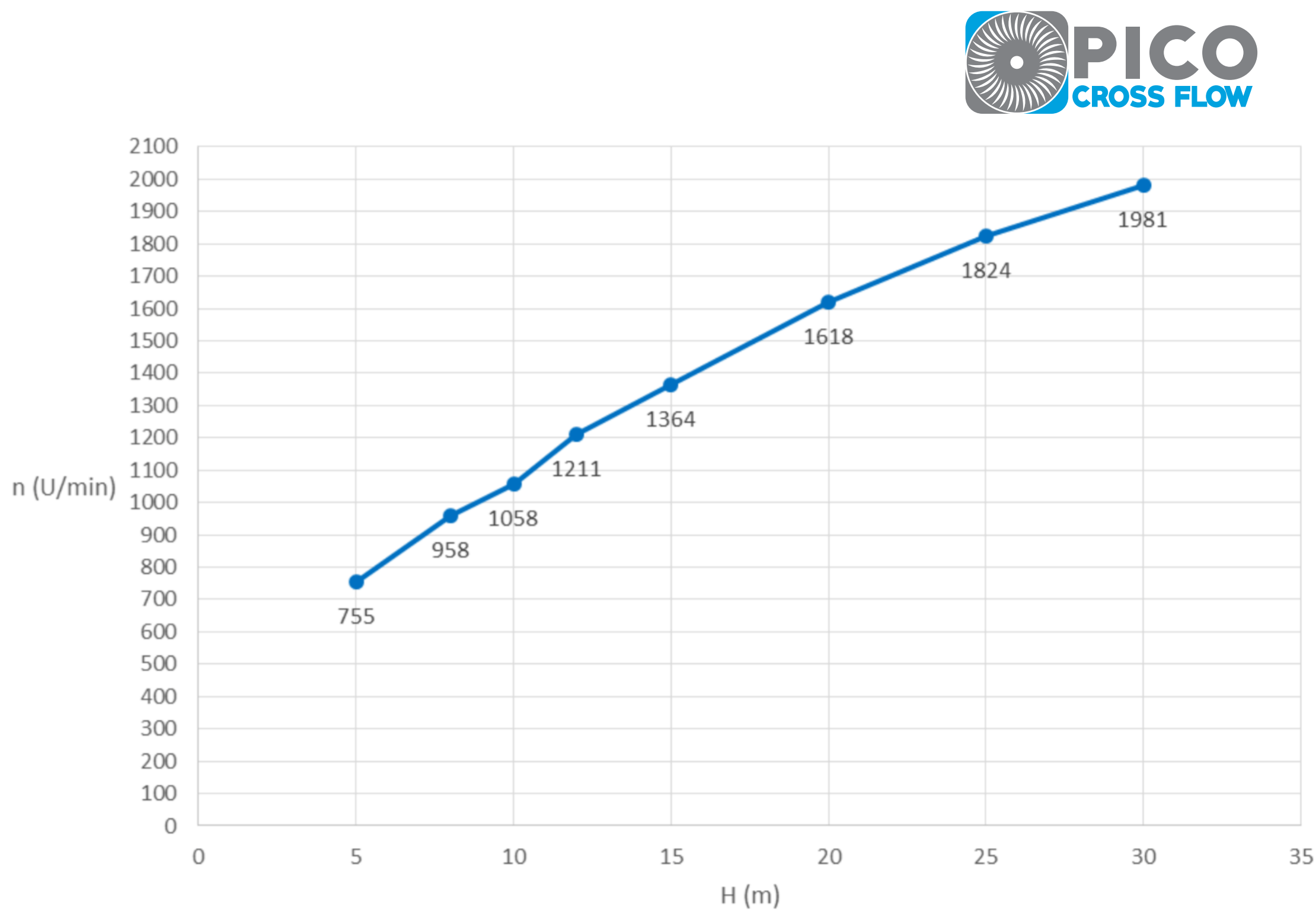
Runaway Speed:

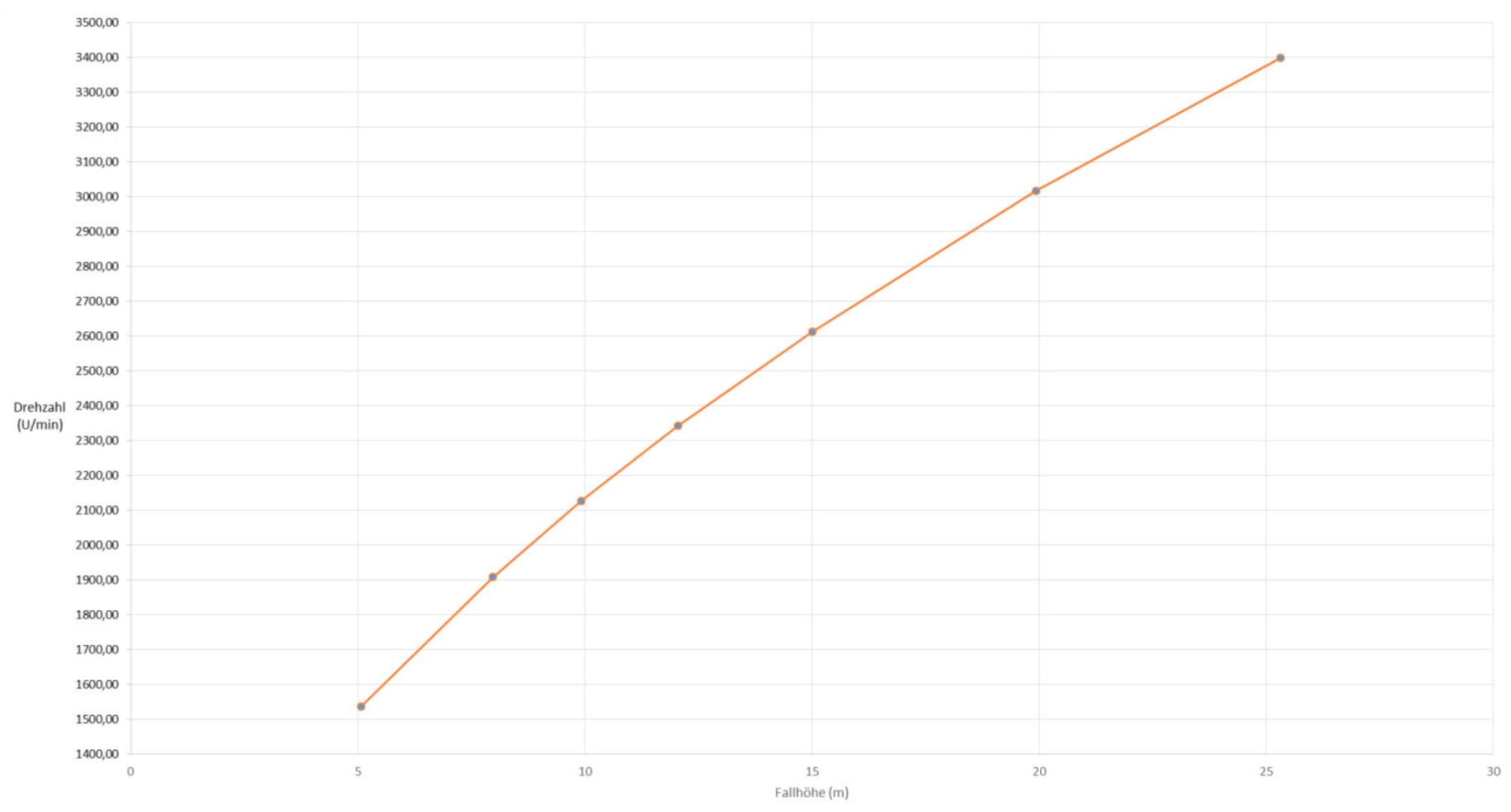




\begin{tabular}{|c|c|c|c|c|c|}
\hline $\mathbf{H}(\mathbf{m})$ & $\mathbf{Q}(\mathbf{m} \mathbf{3} / \mathbf{s})$ & $\mathbf{M}(\mathbf{N m})$ & $\mathbf{n}(\mathbf{U} / \mathbf{m i n})$ & $\mathbf{M} \mathbf{( N m})$ & $\mathbf{P}(\mathbf{k W})$ \\
\hline 5,06 & 0,013 & 0,218 & 1350,9 & 0,22 & 0,03 \\
\hline 5,03 & 0,013 & 0,482 & 1301,0 & 0,48 & 0,07 \\
\hline 5,01 & 0,014 & 0,778 & 1251,2 & 0,78 & 0,10 \\
\hline 5,00 & 0,014 & 1,116 & 1201,3 & 1,12 & 0,14 \\
\hline 4,99 & 0,014 & 1,424 & 1151,9 & 1,42 & 0,17 \\
\hline 4,95 & 0,014 & 1,713 & 1102,0 & 1,71 & 0,20 \\
\hline 5,03 & 0,015 & 2,271 & 1052,3 & 2,27 & 0,25 \\
\hline 5,02 & 0,015 & 2,714 & 1002,8 & 2,71 & 0,28 \\
\hline 5,02 & 0,016 & 3,252 & 953,1 & 3,25 & 0,32 \\
\hline 4,99 & 0,016 & 3,744 & 903,5 & 3,74 & 0,35 \\
\hline 5,00 & 0,017 & 4,326 & 854,0 & 4,33 & 0,39 \\
\hline 5,03 & 0,017 & 4,976 & 804,7 & 4,98 & 0,42 \\
\hline 5,01 & 0,018 & 5,425 & 755,0 & 5,42 & 0,43 \\
\hline 5,00 & 0,018 & 5,659 & 704,9 & 5,66 & 0,42 \\
\hline 5,00 & 0,018 & 5,853 & 655,1 & 5,85 & 0,40 \\
\hline 5,01 & 0,018 & 6,097 & 605,2 & 6,10 & 0,39 \\
\hline 5,02 & 0,018 & 6,387 & 555,4 & 6,39 & 0,37 \\
\hline 5,01 & 0,018 & 6,797 & 505,6 & 6,80 & 0,36 \\
\hline 8,04 & 0,024 & 12,781 & 510,3 & 12,78 & 0,68 \\
\hline 8,03 & 0,023 & 12,021 & 559,9 & 12,02 & 0,70 \\
\hline 8,02 & 0,023 & 11,511 & 609,5 & 11,51 & 0,73 \\
\hline 8,05 & 0,023 & 10,956 & 659,1 & 10,96 & 0,76 \\
\hline 8,01 & 0,023 & 10,434 & 708,9 & 10,43 & 0,77 \\
\hline 8,02 & 0,023 & 10,108 & 758,8 & 10,11 & 0,80 \\
\hline 8,05 & 0,023 & 9,770 & 808,6 & 9,77 & 0,83 \\
\hline 8,06 & 0,023 & 9,531 & 858,6 & 9,53 & 0,86 \\
\hline 8,07 & 0,023 & 9,274 & 908,5 & 9,27 & 0,88 \\
\hline 8,01 & 0,022 & 8,859 & 958,1 & 8,86 & 0,89 \\
\hline 8,04 & 0,022 & 8,360 & 1007,6 & 8,36 & 0,88 \\
\hline 8,02 & 0,021 & 7,521 & 1056,8 & 7,52 & 0,83 \\
\hline 8,04 & 0,021 & 6,890 & 1106,3 & 6,89 & 0,80 \\
\hline
\end{tabular}




\begin{tabular}{|c|c|c|c|c|c|}
\hline 8,00 & 0,020 & 6,087 & 1155,7 & 6,09 & 0,74 \\
\hline 8,02 & 0,020 & 5,475 & 1205,1 & 5,48 & 0,69 \\
\hline 8,02 & 0,020 & 4,858 & 1254,7 & 4,86 & 0,64 \\
\hline 8,02 & 0,019 & 4,310 & 1303,9 & 4,31 & 0,59 \\
\hline 8,02 & 0,019 & 3,786 & 1353,5 & 3,79 & 0,54 \\
\hline 8,02 & 0,018 & 3,302 & 1403,1 & 3,30 & 0,49 \\
\hline 8,03 & 0,018 & 2,814 & 1452,6 & 2,81 & 0,43 \\
\hline 8,01 & 0,018 & 2,368 & 1502,3 & 2,37 & 0,37 \\
\hline 10,05 & 0,018 & 0,663 & 1899,2 & 0,66 & 0,13 \\
\hline 10,01 & 0,018 & 0,947 & 1849,3 & 0,95 & 0,18 \\
\hline 10,02 & 0,019 & 1,342 & 1799,5 & 1,34 & 0,25 \\
\hline 10,01 & 0,019 & 1,751 & 1749,9 & 1,75 & 0,32 \\
\hline 10,03 & 0,019 & 2,215 & 1700,2 & 2,21 & 0,39 \\
\hline 10,02 & 0,019 & 2,600 & 1650,6 & 2,60 & 0,45 \\
\hline 10,00 & 0,020 & 3,016 & 1601,2 & 3,02 & 0,51 \\
\hline 10,01 & 0,020 & 3,540 & 1551,7 & 3,54 & 0,58 \\
\hline 10,01 & 0,020 & 4,183 & 1502,2 & 4,18 & 0,66 \\
\hline 10,01 & 0,021 & 4,722 & 1452,8 & 4,72 & 0,72 \\
\hline 10,01 & 0,021 & 5,237 & 1403,3 & 5,24 & 0,77 \\
\hline 10,01 & 0,022 & 5,818 & 1353,7 & 5,82 & 0,82 \\
\hline 10,01 & 0,022 & 6,515 & 1304,4 & 6,52 & 0,89 \\
\hline 9,99 & 0,023 & 7,332 & 1255,2 & 7,33 & 0,96 \\
\hline 10,01 & 0,023 & 8,050 & 1205,8 & 8,05 & 1,02 \\
\hline 10,02 & 0,024 & 8,827 & 1156,5 & 8,83 & 1,07 \\
\hline 10,02 & 0,024 & 9,730 & 1107,2 & 9,73 & 1,13 \\
\hline 10,01 & 0,025 & 10,466 & 1057,8 & 10,47 & 1,16 \\
\hline 10,00 & 0,025 & 10,818 & 1008,0 & 10,82 & 1,14 \\
\hline 10,00 & 0,025 & 11,101 & 958,6 & 11,10 & 1,11 \\
\hline 10,00 & 0,025 & 11,364 & 908,7 & 11,36 & 1,08 \\
\hline 10,00 & 0,025 & 11,614 & 858,7 & 11,61 & 1,04 \\
\hline 10,02 & 0,025 & 12,137 & 809,0 & 12,14 & 1,03 \\
\hline 10,00 & 0,025 & 12,506 & 759,2 & 12,51 & 0,99 \\
\hline 10,02 & 0,025 & 13,143 & 709,6 & 13,14 & 0,98 \\
\hline
\end{tabular}




\begin{tabular}{|c|c|c|c|c|c|}
\hline 10,00 & 0,025 & 13,725 & 659,9 & 13,72 & 0,95 \\
\hline 10,01 & 0,026 & 14,451 & 610,5 & 14,45 & 0,92 \\
\hline 9,99 & 0,026 & 15,053 & 560,9 & 15,05 & 0,88 \\
\hline 10,00 & 0,026 & 15,896 & 511,2 & 15,90 & 0,85 \\
\hline 11,68 & 0,028 & 18,182 & 614,1 & 18,18 & 1,17 \\
\hline 11,63 & 0,028 & 18,953 & 564,0 & 18,95 & 1,12 \\
\hline 11,68 & 0,028 & 18,546 & 589,2 & 18,55 & 1,14 \\
\hline 11,67 & 0,028 & 17,938 & 639,0 & 17,94 & 1,20 \\
\hline 11,67 & 0,028 & 17,697 & 663,8 & 17,70 & 1,23 \\
\hline 11,68 & 0,028 & 16,844 & 713,2 & 16,84 & 1,26 \\
\hline 11,70 & 0,028 & 16,145 & 762,9 & 16,15 & 1,29 \\
\hline 11,72 & 0,028 & 15,531 & 812,6 & 15,53 & 1,32 \\
\hline 11,78 & 0,028 & 14,517 & 912,0 & 14,52 & 1,39 \\
\hline 11,83 & 0,027 & 13,795 & 1011,9 & 13,79 & 1,46 \\
\hline 11,83 & 0,027 & 13,094 & 1111,0 & 13,09 & 1,52 \\
\hline 11,88 & 0,027 & 12,387 & 1210,6 & 12,39 & 1,57 \\
\hline 12,05 & 0,026 & 10,877 & 1311,4 & 10,88 & 1,49 \\
\hline 12,21 & 0,026 & 9,518 & 1408,5 & 9,52 & 1,40 \\
\hline 12,34 & 0,025 & 8,140 & 1506,9 & 8,14 & 1,28 \\
\hline 15,02 & 0,029 & 12,482 & 1511,7 & 12,48 & 1,98 \\
\hline 15,02 & 0,029 & 13,477 & 1462,6 & 13,48 & 2,06 \\
\hline 15,01 & 0,030 & 14,415 & 1413,5 & 14,41 & 2,13 \\
\hline 15,03 & 0,030 & 15,367 & 1364,3 & 15,37 & 2,20 \\
\hline 15,01 & 0,031 & 15,775 & 1314,7 & 15,78 & 2,17 \\
\hline 15,01 & 0,031 & 16,191 & 1264,9 & 16,19 & 2,14 \\
\hline 14,98 & 0,031 & 16,420 & 1215,4 & 16,42 & 2,09 \\
\hline 15,02 & 0,031 & 16,880 & 1165,7 & 16,88 & 2,06 \\
\hline 15,01 & 0,031 & 17,262 & 1115,8 & 17,26 & 2,02 \\
\hline 15,00 & 0,031 & 17,919 & 1066,2 & 17,92 & 2,00 \\
\hline 15,04 & 0,031 & 18,217 & 1016,5 & 18,22 & 1,94 \\
\hline 15,03 & 0,031 & 18,856 & 966,8 & 18,86 & 1,91 \\
\hline 15,03 & 0,032 & 19,688 & 917,4 & 19,69 & 1,89 \\
\hline 15,03 & 0,032 & 20,692 & 868,1 & 20,69 & 1,88 \\
\hline & & & & & \\
\hline
\end{tabular}




\begin{tabular}{|c|c|c|c|c|c|}
\hline 15,04 & 0,032 & 21,513 & 818,7 & 21,51 & 1,84 \\
\hline 15,01 & 0,032 & 22,236 & 769,0 & 22,24 & 1,79 \\
\hline 15,03 & 0,032 & 23,063 & 719,2 & 23,06 & 1,74 \\
\hline 15,02 & 0,032 & 23,818 & 669,4 & 23,82 & 1,67 \\
\hline 15,02 & 0,032 & 24,732 & 619,7 & 24,73 & 1,60 \\
\hline 15,03 & 0,033 & 25,833 & 570,0 & 25,83 & 1,54 \\
\hline 15,01 & 0,033 & 27,048 & 520,3 & 27,05 & 1,47 \\
\hline 20,04 & 0,031 & 11,912 & 2010,6 & 11,91 & 2,51 \\
\hline 20,01 & 0,032 & 12,857 & 1961,5 & 12,86 & 2,64 \\
\hline 20,01 & 0,032 & 13,549 & 1912,1 & 13,55 & 2,71 \\
\hline 20,05 & 0,032 & 14,705 & 1863,2 & 14,71 & 2,87 \\
\hline 20,02 & 0,033 & 15,635 & 1814,0 & 15,63 & 2,97 \\
\hline 20,01 & 0,033 & 16,800 & 1765,1 & 16,80 & 3,11 \\
\hline 20,00 & 0,034 & 17,855 & 1716,2 & 17,85 & 3,21 \\
\hline 19,92 & 0,034 & 18,937 & 1667,1 & 18,94 & 3,31 \\
\hline 20,05 & 0,035 & 20,602 & 1618,4 & 20,60 & 3,49 \\
\hline 20,04 & 0,035 & 21,065 & 1568,8 & 21,06 & 3,46 \\
\hline 20,06 & 0,035 & 21,650 & 1519,1 & 21,65 & 3,44 \\
\hline 20,03 & 0,036 & 21,931 & 1469,4 & 21,93 & 3,37 \\
\hline 20,01 & 0,036 & 22,245 & 1419,6 & 22,25 & 3,31 \\
\hline 20,01 & 0,036 & 22,596 & 1370,1 & 22,60 & 3,24 \\
\hline 20,04 & 0,036 & 23,116 & 1320,3 & 23,12 & 3,20 \\
\hline 19,98 & 0,036 & 23,711 & 1270,7 & 23,71 & 3,16 \\
\hline 20,03 & 0,036 & 24,401 & 1221,2 & 24,40 & 3,12 \\
\hline 20,04 & 0,036 & 24,895 & 1171,6 & 24,89 & 3,05 \\
\hline 20,05 & 0,037 & 27,025 & 1123,2 & 27,02 & 3,18 \\
\hline 20,05 & 0,037 & 28,095 & 1074,1 & 28,10 & 3,16 \\
\hline 20,00 & 0,037 & 28,484 & 1024,4 & 28,48 & 3,06 \\
\hline 19,99 & 0,037 & 29,619 & 975,0 & 29,62 & 3,02 \\
\hline 20,05 & 0,037 & 30,520 & 925,4 & 30,52 & 2,96 \\
\hline 20,00 & 0,037 & 31,338 & 875,7 & 31,34 & 2,87 \\
\hline 20,02 & 0,037 & 32,283 & 826,0 & 32,28 & 2,79 \\
\hline 20,00 & 0,038 & 33,104 & 776,2 & 33,10 & 2,69 \\
\hline & & & & & \\
\hline
\end{tabular}




\begin{tabular}{|l|l|l|l|l|l|}
\hline 20,05 & 0,038 & 34,196 & 726,8 & 34,20 & 2,60 \\
\hline 20,08 & 0,038 & 34,964 & 676,8 & 34,96 & 2,48 \\
\hline 20,11 & 0,038 & 35,736 & 626,8 & 35,74 & 2,35 \\
\hline 19,98 & 0,038 & 36,945 & 576,8 & 36,95 & 2,23 \\
\hline 19,95 & 0,038 & 37,834 & 526,7 & 37,83 & 2,09 \\
\hline 25,03 & 0,037 & 20,740 & 2020,8 & 20,74 & 4,39 \\
\hline 25,03 & 0,037 & 21,950 & 1972,0 & 21,95 & 4,53 \\
\hline 24,99 & 0,038 & 22,928 & 1921,7 & 22,93 & 4,61 \\
\hline 24,97 & 0,038 & 24,343 & 1872,9 & 24,34 & 4,77 \\
\hline 24,99 & 0,039 & 25,914 & 1824,4 & 25,91 & 4,95 \\
\hline 25,02 & 0,039 & 26,636 & 1775,1 & 26,64 & 4,95 \\
\hline 25,00 & 0,040 & 27,197 & 1725,5 & 27,20 & 4,91 \\
\hline 25,01 & 0,040 & 27,681 & 1675,9 & 27,68 & 4,86 \\
\hline 25,00 & 0,040 & 28,055 & 1626,3 & 28,06 & 4,78 \\
\hline 24,98 & 0,040 & 28,517 & 1576,6 & 28,52 & 4,71 \\
\hline 25,04 & 0,040 & 29,093 & 1527,0 & 29,09 & 4,65 \\
\hline 25,01 & 0,040 & 29,534 & 1477,3 & 29,53 & 4,57 \\
\hline 25,02 & 0,040 & 30,242 & 1427,9 & 30,24 & 4,52 \\
\hline 24,96 & 0,040 & 30,788 & 1378,4 & 30,79 & 4,44 \\
\hline 25,00 & 0,040 & 31,428 & 1328,9 & 31,43 & 4,37 \\
\hline 25,00 & 0,040 & 32,116 & 1279,6 & 32,12 & 4,30 \\
\hline 25,04 & 0,041 & 33,390 & 1230,6 & 33,39 & 4,30 \\
\hline 25,01 & 0,041 & 34,735 & 1181,6 & 34,73 & 4,30 \\
\hline 25,01 & 0,042 & 36,203 & 1132,3 & 36,20 & 4,29 \\
\hline 25,03 & 0,042 & 37,470 & 1083,1 & 37,47 & 4,25 \\
\hline 25,01 & 0,042 & 38,654 & 1033,7 & 38,65 & 4,18 \\
\hline 25,02 & 0,042 & 39,654 & 984,4 & 39,65 & 4,09 \\
\hline 25,01 & 0,042 & 40,580 & 934,6 & 40,58 & 3,97 \\
\hline 25,00 & 0,042 & 41,709 & 884,9 & 41,71 & 3,86 \\
\hline 25,01 & 0,043 & 43,105 & 835,4 & 43,11 & 3,77 \\
\hline 25,05 & 0,043 & 44,350 & 785,7 & 44,35 & 3,65 \\
\hline 25,03 & 0,042 & 44,434 & 735,2 & 44,43 & 3,42 \\
\hline 25,02 & 0,042 & 44,942 & 684,8 & 44,94 & 3,22 \\
\hline
\end{tabular}




\begin{tabular}{|l|l|l|l|l|l|}
\hline 25,00 & 0,042 & 46,288 & 634,9 & 46,29 & 3,08 \\
\hline 24,95 & 0,043 & 47,780 & 585,1 & 47,78 & 2,93 \\
\hline 24,99 & 0,043 & 49,470 & 535,2 & 49,47 & 2,77 \\
\hline 30,03 & 0,041 & 27,371 & 2128,3 & 27,37 & 6,10 \\
\hline 30,05 & 0,042 & 28,729 & 2079,5 & 28,73 & 6,26 \\
\hline 30,01 & 0,042 & 30,133 & 2030,0 & 30,13 & 6,41 \\
\hline 30,05 & 0,043 & 31,286 & 1981,0 & 31,29 & 6,49 \\
\hline 30,02 & 0,043 & 31,932 & 1931,6 & 31,93 & 6,46 \\
\hline 29,99 & 0,043 & 32,481 & 1882,1 & 32,48 & 6,40 \\
\hline 30,02 & 0,043 & 32,867 & 1832,6 & 32,87 & 6,31 \\
\hline 30,02 & 0,044 & 33,393 & 1783,0 & 33,39 & 6,23 \\
\hline 30,01 & 0,044 & 33,954 & 1733,3 & 33,95 & 6,16 \\
\hline 30,00 & 0,044 & 34,448 & 1683,7 & 34,45 & 6,07 \\
\hline 29,99 & 0,044 & 35,108 & 1634,1 & 35,11 & 6,01 \\
\hline 30,06 & 0,044 & 35,921 & 1584,7 & 35,92 & 5,96 \\
\hline 29,99 & 0,044 & 36,567 & 1535,2 & 36,57 & 5,88 \\
\hline 30,03 & 0,044 & 37,294 & 1485,9 & 37,29 & 5,80 \\
\hline 29,97 & 0,044 & 37,742 & 1436,3 & 37,74 & 5,68 \\
\hline 30,00 & 0,044 & 38,571 & 1386,7 & 38,57 & 5,60 \\
\hline 30,00 & 0,045 & 39,842 & 1337,8 & 39,84 & 5,58 \\
\hline 30,08 & 0,045 & 41,792 & 1289,0 & 41,79 & 5,64 \\
\hline 30,03 & 0,045 & 43,179 & 1239,9 & 43,18 & 5,61 \\
\hline 30,01 & 0,046 & 44,425 & 1190,7 & 44,43 & 5,54 \\
\hline 30,02 & 0,046 & 45,792 & 1141,3 & 45,79 & 5,47 \\
\hline 30,08 & 0,046 & 47,217 & 1092,0 & 47,22 & 5,40 \\
\hline 29,97 & 0,046 & 48,095 & 1042,2 & 48,09 & 5,25 \\
\hline 30,04 & 0,046 & 49,402 & 992,7 & 49,40 & 5,14 \\
\hline 29,98 & 0,046 & 50,494 & 943,0 & 50,49 & 4,99 \\
\hline 30,07 & 0,047 & 52,339 & 893,8 & 52,34 & 4,90 \\
\hline 30,01 & 0,047 & 53,497 & 844,1 & 53,50 & 4,73 \\
\hline 30,06 & 0,046 & 53,638 & 793,4 & 53,64 & 4,46 \\
\hline 30,09 & 0,046 & 54,363 & 743,0 & 54,36 & 4,23 \\
\hline 30,04 & 0,046 & 55,461 & 692,6 & 55,46 & 4,02 \\
\hline
\end{tabular}




\begin{tabular}{|l|l|l|l|l|l|}
\hline 30,01 & 0,047 & 57,100 & 642,7 & 57,10 & 3,84 \\
\hline 30,01 & 0,047 & 58,873 & 592,7 & 58,87 & 3,65 \\
\hline 30,00 & 0,047 & 60,591 & 542,5 & 60,59 & 3,44 \\
\hline
\end{tabular}

